\title{
Análise e implementação de métodos implícitos no sistema FreeFlow2 ${ }^{1}$
}

\author{
Cassio Machiaveli Oishi
}

Orientador: Prof. Dr. José Alberto Cuminato

Dissertação apresentada ao Instituto de Ciências Matemáticas e de Computação - ICMC-USP, como parte dos requisitos para obtenção do título de Mestre em Ciências - Ciências da Computação e Matemática Computacional.

\section{"VERSÃO REVISADA APÓS A DEFESA"}

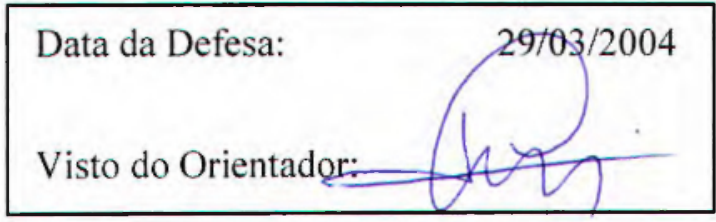

USP - São Carlos

Maio/2004

1 Este trabalho teve o suporte financeiro da FAPESP proc.:01/12540-2 
Dedico às pessoas que mais amo na vida:

minha família. 


\section{Agradecimentos}

Primeiro a Deus pela minha vida.

Ao professor José Alberto Cuminato pela orientação e paciência.

Aos professores Valdemir Garcia Ferreira, Norberto Mangiavacchi, Hélio Aparecido Navarro, Fernando Marques Federson, Murilo Tomé e Antonio Castelo Filho pelas discussões e convivência.

A todos meus amigos, em especial, aos amigos João Paulo, Olivâine e Samuel, e as amigas Dayene, Gilcilene, Kemelli e Andrea. Aos novos amigos Billy, Igor, Eduardo, Alex, Helton, Fabrício, Marcelo, Fred, Silvino e a todos do LCAD.

E finalmente a todos que contribuíram para realização deste trabalho 


\section{Sumário}

1 Introdução 1

2 Métodos Numéricos para Equações Diferenciais: Conceitos Básicos 7

2.1 Introdução . . . . . . . . . . . . . . . . 7

2.2 Aproximação por Diferenças Finitas . . . . . . . . . . . . . 8

2.2.1 Aplicação do Método de Diferenças Finitas para EDPs . . . . . 10

2.3 Consistência . . . . . . . . . . . . . . . . . . 12

2.4 Estabilidade . . . . . . . . . . . . . . . . . . . 13

2.4.1 Critério de Von Neumann . . . . . . . . . . . . . 14

2.4 .2 Critério da Matriz . . . . . . . . . . . . . . . 17

2.5 A-Estabilidade . . . . . . . . . . . . . . . . . 18

2.5.1 Métodos A-estáveis . . . . . . . . . . . . . . . 19

2.6 Convergência . . . . . . . . . . . . . . . 23

3 Equações de Navier-Stokes: Derivação e Condições de Fronteira 25

3.1 Introdução . . . . . . . . . . . . . . . . . 25

3.2 Descrição do Movimento de Fluido . . . . . . . . . . . . 26

3.3 Equações da Mecânica do Contínuo . . . . . . . . . . . . . . . . 27

3.4 Fluidos Newtonianos . . . . . . . . . . . . . . . . . . . . 29

3.5 Adimensionalização . . . . . . . . . . . . . . . . 31

3.6 Escoamentos Viscosos . . . . . . . . . . . . . . . . . 33

3.7 Condições Iniciais e de Contorno . . . . . . . . . . . . . 33

4 Método de Projeção e Formulações Implícitas para Equações de NavierStokes 37

4.1 Introdução . . . . . . . . . . . . . . . . . . . 37

4.2 Métodos de Projeção . . . . . . . . . . . . . . . . . . . . . . 40

4.2.1 Teorema da Decomposição de Helmholtz-Hodge . . . . . . . . . 41

4.2.2 Aplicação do Teorema da DHH para Métodos de Projeção . . . 42

4.2.3 Aplicação do Teorema DHH na Forma Discreta para o Método MAC ......................... 46

4.3 Formulações Implícitas para Equaçōes de Navier-Stokes . . . . . . . . 47 
5 Formulações Implícitas na Metodologia GENSMAC 51

5.1 Introdução . . . . . . . . . . . . . . . . . . 51

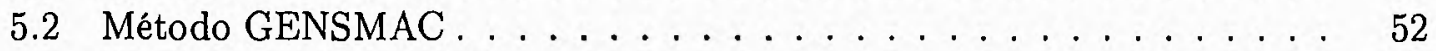

5.2 .1 Classificação das Células . . . . . . . . . . . . . . . . 54

5.2 .2 Condições Iniciais e de Contorno . . . . . . . . . . . . 56

5.2 .3 Algoritmo do Método GENSMAC . . . . . . . . . . . . . 56

5.2.4 Condição de Estabilidade do Método GENSMAC . . . . . . . . 58

5.3 Método de Projeção-1 para Formulações Implícitas na metodologia GENS-

MAC . . . . . . . . . . . . . . . . . . 59

5.3.1 Condições Iniciais e de Contorno para o método de Projeção-1 . 61

5.3.2 Algoritmo do Método de Projeção-1 utilizando Formulações Implícitas . . . . . . . . . . . . . . . . 66 66

5.3.3 Condição de Estabilidade do Método de Projeção-1 utilizando Formulações Implícitas . . . . . . . . . . . . . . . . 67

5.4 Método de Projeção-2 para Formulações Implícitas na metodologia GENSMAC . . . . . . . . . . . . . . . . . . 69

5.4.1 Condições Iniciais e de Contorno para o método de Projeção-2 . 70

5.4.2 Algoritmo do Método de Projeção-2 utilizando Formulações Implícitas . . . . . . . . . . . . . . . 73

5.4.3 Condição de Estabilidade do Método de Projeção-2 utilizando Formulações Implícitas . . . . . . . . . . . . . . 74

5.5 Método dos Gradientes Bi-conjugados para Sistemas Esparsos . . . . . 74

6 Discretização das Equações e Ambiente FreeFlow2D $\quad 79$

6.1 Introdução . . . . . . . . . . . . . . . . . . . . 79

6.2 Discretização das Equações . . . . . . . . . . . . . . . . . . . . 80

6.2.1 Discretização das Equações de Conservação . . . . . . . . . . . . . . 81

6.2.2 Discretização dos Termos Convectivos . . . . . . . . . . . . 85

6.2.3 Discretização das Equações para o Potencial $\psi$. . . . . . . . . 86

6.2 .4 Técnicas de Armazenagem . . . . . . . . . . . . . . . . 91

6.2.5 Discretização das Equações de Atualização . . . . . . . . . . . . 92

6.3 Ambiente de Simulação FreeFlow2D . . . . . . . . . . . . . . . 93

6.3 .1 Modelador . . . . . . . . . . . . . . . . 93

6.3 .2 Simulador . . . . . . . . . . . . . . . . 94

6.3 .3 Visualizador . . . . . . . . . . . . . . . 95

6.3 .4 Re-inicializador . . . . . . . . . . . . . 96

$\begin{array}{lll}7 & \text { Resultados Numéricos } & \mathbf{9 7}\end{array}$

7.1 Introdução . . . . . . . . . . . . . . . . . . . . . . 97

7.2 Simulação do Escoamento em um Canal . . . . . . . . . . . . . . . 98

7.3 Comparação com a Solução Aproximada de Watson . . . . . . . . . . 112

7.4 Simulação Numérica do Preenchimento de uma Caixa . . . . . . . . . . . 117

7.5 Simulação Numérica do Preenchimento de uma Caixa contendo Fluido 121

7.6 Comparação com Resultados Experimentais . . . . . . . . . . . . . 123 
8 Conclusões e Futuros Trabalhos $\quad 133$

8.1 Síntese do Trabalho . . . . . . . . . . . . . . . . . 133

8.2 Considerações Finais sobre os Resultados . . . . . . . . . . . . . . . 134

8.3 Contribuição . . . . . . . . . . . . . . . . . . . . 136

8.4 Futuros Trabalhos . . . . . . . . . . . . . . . . . . 136

$\begin{array}{lr}\text { Referências Bibliográficas } & 149\end{array}$ 
2.1 Região de estabilidade para $\theta=0 \ldots \ldots \ldots \ldots . \ldots \ldots 21$

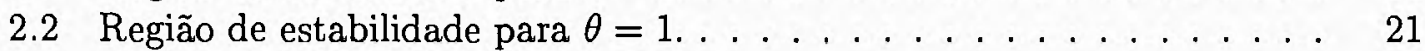

2.3 Região de estabilidade para $\theta=1 / 2 \ldots \ldots \ldots \ldots \ldots 22$

2.4 Teorema de Lax. . . . . . . . . . . . . . . . . . 23

3.1 Diagrama de Conservação de Massa. . . . . . . . . . . . . . . . . 28

3.2 Diagrama de Conservação de Movimento. . . . . . . . . . . . . . . . . 29

3.3 Exemplo da força de cisalhamento atuando sobre um fluido. . . . . . . 30

3.4 Componentes das velocidades em uma fronteira. . . . . . . . . . . 34

4.1 Célula utilizada pelo método MAC. . . . . . . . . . . . . . . . . . 40

5.1 Tipos de células. . . . . . . . . . . . . . . . 55

5.2 Configuração de uma célula computacional mostrando uma célula da superfície livre em contato, na sua face lateral direita, com uma célula vazia. . . . . . . . . . . . . . . . . .

5.3 Configuração de uma célula computacional mostrando uma célula da superfície livre em contato, na sua face superior, com uma célula vazia.

5.4 Configuração de uma célula computacional mostrando uma célula da superfície livre e que possui duas célula vazia adjacentes. . . . . . . . .

5.5 Configuração de uma célula computacional mostrando uma célula da superfície livre e que possui duas célula vazia adjacentes. . . . . . . . .

5.6 Configuração de uma célula computacional mostrando uma célula da superfície livre em contato, na sua face lateral esquerda, com uma célula vazia. . . . . . . . . . . . . . . . . . .

5.7 Configuração de uma célula computacional, mostrando uma célula da superfície livre em contato, na sua face inferior, com uma cẻlula vazia. .

5.8 Configuração de uma célula computacional mostrando uma célula da superfície livre e que possui duas célula vazia adjacentes. . . . . . . . .

5.9 Configuração de uma célula computacional mostrando uma célula da superfície livre e que possui duas célula vazia adjacentes. . . . . . . 72

6.1 Processo de Discretização. . . . . . . . . . . . . . . . . . . 81

6.2 Configuração de células discretizadas - Caso 1. . . . . . . . . 87 
6.3 Configuração de células discretizadas - Caso $2 . \ldots \ldots$. . . . . . . 88

6.4 Configuração de células discretizadas - Caso $3 . \ldots \ldots$. . . . . . . . . 89

6.5 Configuração de células discretizadas - Caso 4. . . . . . . . . . . 91

6.6 Interface gráfica do modelador. . . . . . . . . . . . . . . . . 94

6.7 Interface gráfica do visualizador. . . . . . . . . . . . . . . 95

6.8 Interface gráfica do re-inicializador. . . . . . . . . . . . 96

7.1 Domínio para um escoamento de Hagen-Poiseuille. O escoamento é da esquerda para a direita e o comprimento do canal é $5 L$. . . . . . . . . .

7.2 Simulação numérica do escoamento de Hagen-Poiseuille. Superfície do fluido nos tempos: a) $t=2.0 \mathrm{~s}, \mathrm{~b}) t=6.0 \mathrm{~s}$ e c) $t=12.0 \mathrm{~s}$. . . . . . .

7.3 Comparação entre soluções numéricas obtida pelo método Projeção-1 utilizando FI e a solução analítica dada pela equação (7.11), sobre as três malhas, com $R e=0.1$. As figuras a)-c) correspondem ao método Projeção-1 utilizando as formulações IR, CN e AB/CN, respectivamente. 103

7.4 Comparação entre soluções numéricas obtida pelo método Projeção-2 utilizando FI e a solução analítica dada pela equação (7.11), sobre as três malhas, com $R e=0.1$. As figuras a)-c) correspondem ao método Projeção-2 utilizando as formulações IR, CN e AB/CN, respectivamente. 104

7.5 Erro relativo na norma $l_{2}$, para a velocidade $u$ e os métodos Projeção-1 e Projeção-2 utilizando a formulação IR sobre a malha M2. . . . . . 106

7.6 Erro relativo na norma $l_{2}$, para a velocidade $u$ e os métodos Projeção-1 e Projeção-2 utilizando a formulação CN sobre a malha M2. . . . . . . 106

7.7. Erro relativo na norma $l_{2}$, para a velocidade $u$ e os métodos Projeção- 1 e Projeção-2 utilizando a formulaçã̃o $\mathrm{AB} / \mathrm{CN}$ sobre a malha M2. . . .

7.8 Definição do domínio para o escoamento de um jato livre sobre uma superfície rígida impermeável. . . . . . . . . . . . . . .

7.9 Superfície livre de um jato de fluido incidindo sobre uma superfície rígida impermeável.

7.10 Comparação entre soluções numéricas obtida pelo método Projeção-1 utilizando FI e a solução analítica de Watson, com $R e=5000$. As figuras a)-c) correspondem ao método Projeção-1 utilizando as formulações IR, $\mathrm{CN}$ e $\mathrm{AB} / \mathrm{CN}$, respectivamente. . . . . . . . . . . . . . . . . .

7.11 Comparação entre soluções numéricas obtida pelo método Projeção-2 utilizando FI e a solução analítica de Watson, com $R e=5000$. As figuras a)-c) correspondem ao método Projeção-2 utilizando as formulações IR, $\mathrm{CN}$ e $\mathrm{AB} / \mathrm{CN}$, respectivamente.

7.12 Domínio para o problema do preenchimento de uma caixa. . . . . . . 117

7.13 Resultados numéricos para a simulação do preenchimento de uma caixa, com $R e=0.1$ e tempo de simulação $t=0.28 \mathrm{~s}$. Métodos: a) Explícito, b)-d) Projeção-1 utilizando as formulações IR, $\mathrm{CN}$ e $\mathrm{AB} / \mathrm{CN}$, respectivamente, e e)-g) Projeção-2 utilizando as formulações IR, CN e AB/CN. 119

7.14 Domínio para o problema do preenchimento de uma caixa contendo fluido. 121 
7.15 Comparação da pressão $p$ para o Modelo 1 - $R e=0.22$ entre as soluções do método explícito (esquerda) e Projeção-2 utilizando a formulação IR (direita). a) $t=0.0125 \mathrm{~s} \mathrm{e} \mathrm{b)} t=0.1375 \mathrm{~s} . \ldots \ldots \ldots \ldots \ldots$. . . . . .

7.16 Comparação para o Modelo $1-R e=0.8$. Solução experimental (esquerda) e numérica (a solução do meio é obtida pelo método Projeção2 utilizando a formulação IR, e a da direita, pelo método explícito). a) $t=0.14 \mathrm{~s}, \mathrm{~b}) t=0.22 \mathrm{~s}, \mathrm{c}) t=0.26 \mathrm{~s}$ e d) $t=0.34 \mathrm{~s} . \ldots \ldots$

17 Comparação entre a velocidade $u$ para o Modelo $1-R e=0.8$, no tempo $t=0.14 \mathrm{~s}$. Métodos: a) Explícito, b)-d) Projeção-1 utilizando as formulações IR, $\mathrm{CN}$ e $\mathrm{AB} / \mathrm{CN}$, respectivamente, e e)-g) Projeção-2 utilizando as formulações IR, $\mathrm{CN}$ e $\mathrm{AB} / \mathrm{CN}$. . . . . . . . . . . . .

7.18 Comparação entre a velocidade $v$ para o Modelo $1-R e=0.8$, no tempo $t=0.14 \mathrm{~s}$. Métodos: a) Explícito, b)-d) Projeção-1 utilizando as formulações IR, $\mathrm{CN}$ e $\mathrm{AB} / \mathrm{CN}$, respectivamente, e e)-g) Projeção-2 utilizando as formulações IR, $\mathrm{CN}$ e $\mathrm{AB} / \mathrm{CN}$. . . . . . . . . . . . .

7.19 Comparação para o Modelo $2-R e=0.15$. Solução experimental (esquerda) e numérica (a solução do meio é obtida pelo método Projeção2 utilizando a formulação $\mathrm{CN}$, e a da direita, pelo método explícito). a) $t=0.37 \mathrm{~s}, \mathrm{~b}) t=0.43 \mathrm{~s}, \mathrm{c}) t=0.47 \mathrm{~s} \mathrm{e} \mathrm{d}) t=0.66 \mathrm{~s}$.

7.20 Comparação entre soluções numéricas obtidas pelo método Projeção-2 utilizando a formulação IR. A coluna esquerda mostra o desenvolvimento do método para $\delta t=6.25 \times 10^{-5} \mathrm{~s}$, e a coluna direita, para $\delta t=2.0 \times 10^{-4} \mathrm{~s}$. As soluções numéricas são obtidas nos tempos: a) $t=0.37 \mathrm{~s}, \mathrm{~b}) t=0.43 \mathrm{~s}$, c) $t=0.47 \mathrm{~s}$ e d) $t=0.66 \mathrm{~s}$. 


\section{Lista de Tabelas}

7.1 Resultados do escoamento de Hagen-Poiseuille para $R e=0.1$ nas malhas M1, M2 e M3. O valor do $\delta t$ é dado em segundos após o escoamento atingir o estado estacionário. . . . . . . . . . . . . . . . . .

7.2 Comparação entre os métodos Projeção-1 e Projeção-2, ambos utilizando a formulação IR, para o erro relativo no escoamento de Hagen-Poiseuille

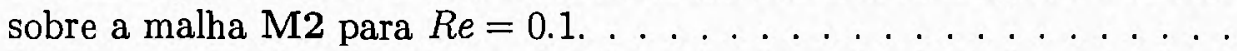

7.3 Comparação entre os métodos Projeção-1 e Projeção-2, ambos utilizando a formulação CN, para o erro relativo no escoamento de Hagen-Poiseuille sobre a malha M2 para $R e=0.1 \ldots \ldots \ldots \ldots \ldots \ldots$

7.4 Comparação entre os métodos Projeção-1 e Projeção-2, ambos utilizando a formulação $\mathrm{AB} / \mathrm{CN}$, para o erro relativo no escoamento de HagenPoiseuille sobre a malha M2 para $R e=0.1 \ldots \ldots \ldots$. . . . .

7.5 Números de equações e iterações dos métodos GC (Explícito) e GBCP (Projeção-1 e Projeção-2 utilizando as FI). Resultados para o escoamento sobre a malha M2.

Limite de estabilidade para $\delta t(\mathrm{~s})$ no escoamento de Hagen-Poiseuille sobre a malha M2, com diferentes valores para $R e . \ldots \ldots \ldots . . .$.

7.7 Resultados para o escoamento de Hagen-Poiseuille para $R e=0.1$, sobre a malha M2, com tempo de simulação $t=20 \mathrm{~s} . \ldots \ldots \ldots \ldots$

7.8 Resultados para o escoamento de Hagen-Poiseuille para $R e=0.01$, sobre a malha M2, com tempo de simulação $t=20$ s. . . . . . . . . . .

7.9 Resultados para o escoamento de Hagen-Poiseuille para $R e=0.001$, sobre a malha M2, com tempo de simulação $t=30$ s. . . . . . . . .

7.10 Resultados para o escoamento de um jato preenchendo um caixa de $L=$ $0.05 \mathrm{~m}, U=1.0 \mathrm{~ms}^{-1}, R e=0.1$ no instante $t=0.28 \mathrm{~s} \ldots \ldots \ldots 120$

7.11 Resultados para o escoamento de um jato preenchendo um caixa de $L=$ $0.05 \mathrm{~m}, U=1.0 \mathrm{~ms}^{-1}, R e=0.01$ no instante $t=0.28 \mathrm{~s} . \ldots \ldots 120$

7.12 Modelo 1 - Re $=0.22, L=0.002 \mathrm{~m}$ e $U=1.0 \mathrm{~ms}^{-1}$. . . . . . . . 123

7.13 Modelo 1 - $R e=0.8, L=0.004 \mathrm{~m}$ e $U=1.0 \mathrm{~ms}^{-1} \ldots \ldots \ldots \ldots$. . . 124

7.14 Modelo 2 - $R e=0.15, L=0.004 \mathrm{~m}$ e $U=1.0 \mathrm{~ms}^{-1} \ldots \ldots \ldots$ 
Neste trabalho, formulações implícitas são adaptadas à metodologia GENSMAC, para a solução numérica de escoamentos bidimensionais, transientes, newtonianos e incompressíveis. Usando a formulação velocidade-pressão e variações do método de projeção, o ambiente de simulação FreeFlow2D é utilizado para resolver numericamente as equações de conservação, no contexto de diferenças finitas. Os termos difusivos nas equações de Navier-Stokes são tratados implicitamente por meio das formulações Implícita Regressiva, Crank-Nicolson e Adams-Bashforth/Crank-Nicolson. Os termos convectivos são tratados explicitamente por um esquema upwind de alta ordem limitado. Para escoamentos em canais e jatos com superfícies livres a baixos números de Reynolds, as formulações são robustas e possibilitam um aumento considerável no tamanho do passo temporal. Resultados numéricos que comparam muito bem com soluções analíticas e experimentais são apresentados. 
In this work, implicit schemes are studied with the purpose of adapting them to the two-dimensional GENSMAC method, for the numerical solution of unsteady newtonian incompressible flows. Using the velocity-pressure formulation and different projection methods, the FreeFlow2D simulation system is employed to solve the conservation equations in the context of finite-difference. The viscous terms in the Navier-Stokes equations are implicitly treated via the Implicit Backward, Crank-Nicolson and AdamsBashforth/Crank-Nicolson schemes. The convective terms are explicitly discretized by an upwind high-order limited scheme. For channel and jet flows with low Reynolds number and with free surfaces, the schemes are robusts and allow for larger time steps. Numerical results that compare very well with the analytic and experimental solutions are shown. 


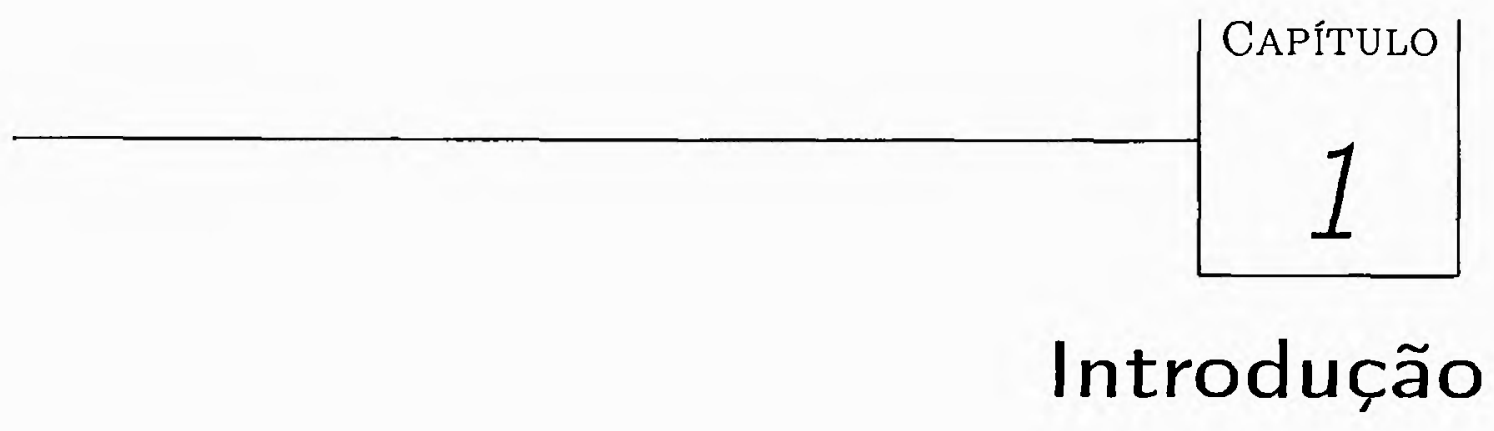

O estudo de fenómenos físicos e leis da natureza é uma atividade que vem sendo desenvolvida há muitos séculos. Cientistas como Aristóteles, Arquimedes, Einstein, Galileu, Leonard Euler, Newton entre outros foram responsáveis por diversas descobertas que motivam a comunidade científica até os dias atuais.

No passado havia dois métodos científicos com ponto de partida para o estudo das leis da natureza: o prático e o teórico. O método prático ocupa-se das observações e experimentos, enquanto o teórico desenvolve leis e teorias físicas.

Atualmente, o desenvolvimento científico tem-se beneficiado enormemente de um novo método científico criado com o objetivo de conectar os métodos prático e teórico: o numérico. O uso de técnicas numéricas para a solução de problemas complexos da engenharia e da física é, hoje, uma realidade, graças ao desenvolvimento de novos algoritmos, de computadores de alta velocidade e de grande capacidade de armazenamento. Em função dessa disponibilidade computacional o desenvolvimento de algoritmos para a solução dos mais diversos problemas tem recebido enorme atenção dos analista numéricos. As principais vantagens das soluções numéricas são: baixo custo, evolução temporal do processo, resolve problemas em geometrias complexas, apresentar resultados com rapidez e etc. Entretanto, a solução numérica também apresenta algumas desvantagens, como: erros de truncamento, instabilidades, prescrição das condições de contorno apropriadas e custos computacionais. Alguns exemplos da penetração de técnicas numéricas na indústria são importantes como motivação. No projeto de aviões, por exemplo, o uso 
de técnicas numéricas diminui, sensivelmente, o trabalho de laboratôrio, economizando também custos financeiros.

Neste trabalho, a atenção é voltada para o estudo das técnicas numéricas em problemas que envolvem escoamentos de fluidos. Líquidos e gases têm em comum, graças à facilidade de deformação, a propriedade de escoar ou fluir facilmente, donde o nome fluido. As descrições matemática do comportamento dos fluidos ganharam força no século XIX, na forma das equações de Navier-Stokes, a partir dos trabalhos pioneiros dos franceses Claude Navier (1822) e Simeon Poisson (1829) e do inglês George Stokes (1845). A solução dos problemas de escoamentos de fluidos requer o manuseio das equações de Navier-Stokes, altamente não-lineares, acopladas às equações da conservação de massa e energia. Em conjunto com condições iniciais e de contorno fisicamente apropriadas, as equações de Navier-Stokes permitem, em principio, obter informações do caráter fundamental da dinâmica dos fluidos. Devido às dificuldades de se obter, na maior parte dos problemas, soluções teôricas para as equações de Navier-Stokes, a solução numérica tornou-se fundamental, nascendo uma importante área: A Dinâmica de Fluidos Computacional (DFC). Essa área estuda métodos computacionais para a simulação de fenômenos que envolvem fluidos em movimento com ou sem troca de calor.

O objetivo básico da DFC é reduzir o número de experimentos e explorar fenômenos que não poderiam ser estudados em laboratôrio de forma prática. Utilizando as técnicas da DFC pode-se avaliar numericamente os diversos parâmetros relevantes do problema. Esses podem ser facilmente alterados até que o resultado da simulação atenda às exigências do projeto. A DFC está envolvida, praticamente, em todos os processos de produção de energia, nos fenômenos ambientais, nos projetos de equipamentos térmicos, na engenharia aeronáutica e aeroespacial, engenharia de reatores, engenharia química, medicina, siderúrgica, indústria textil e injeções em moldes. Essa é somente uma pequena amostra das aplicações da DFC.

Uma importante característica na DFC é classificar o escoamento e o fluido. Desta forma, os escoamentos podem ser classificados de diversas formas, como: estacionários (cujas grandezas como velocidade e pressão não variam com o tempo) ou transientes (oposto do estacionário); internos (escoamentos em que o fluido está cercado por paredes e preenche todo o volume entre elas) ou externos (quando o fluido não está confinado por paredes, podendo apresentar ou não superfícies livres); laminares (são aqueles nos quais camadas muito finas de fluido parecem deslizar umas sobre as outras) ou turbulen- 
tos (quando o escoamento origina um movimento caótico ou desordenado de partículas); incompressiveis (quando a densidade de um fluido não muda ao longo do escoamento ou quando pode-se desprezar qualquer alteração em seu valor) ou compressíveis (oposto do incompressíveis). Do ponto de vista reológico, um fluido escoando é constituído de moléculas, que estão individualmente em um estado de movimento ao acaso e possuem a habilidade de transmitir cisalhamento. Essa propriedade introduz o conceito de viscosidade dinâmica. Quando a tensão cisalhante é diretamente proporcional ao gradiente de velocidade, sendo o coeficiente de viscosidade a constante de proporcionalidade, esses fluidos são conhecidos como newtonianos (White, 1991), (Bretas e D'Avila, 2000). Alguns escoamentos podem modificar sua classificação com a alteração de alguns parâmetros adimensionais. Um parâmetros muito conhecido na literatura que governa a transição de um escoamento laminar para turbulento é o número de Reynolds definido como:

$$
R e=\frac{\text { forças inerciais }}{\text { forças viscosas }}
$$

apresentado por Osborne Reynolds (1883). Os escoamentos onde as forças viscosas têm grande influência são denominados viscosos. Na literatura de DFC, os escoamentos costumam ser definidos em função do seu número de Reynolds, e de outras grandezas adimensionais apropriadas ao problema. De acordo com (Flecther, 1992), alguns valores típicos para o número de Reynolds são: Espermatozóide em movimento ( $R e=0.006$ ), Gotícula d'água caindo $(R e=0.64)$, Boeing 747 em velocidade e altitude de cruzeiro $(R e=7000000)$.

Neste trabalho, o interesse de estudo são as soluções numéricas de escoamentos de fluidos estacionários e transientes, internos e externos, laminares, incompressíveis e viscosos $(R e<<1)$ para fluidos newtonianos.

Nas últimas décadas muitos esforços têm sido dirigidos na obtenção de soluções numéricas de escoamentos de fluidos viscosos incompressíveis. Uma dificuldade adicional aparece se esses escoamentos envolvem superfícies livres, pois a presença desses contornos envolve problemas como: a própria configuração da superfície livre deve ser determinada a cada passo no tempo e o movimento da superfície livre é geralmente influenciado por fenómenos interfaciais. Apesar desses inconvenientes existem vários métodos disponíveis na literatura especializada para se resolver a classe dos problemas com superfícies livres. A maioria desses métodos foram influenciados pelo método MAC (Harlow e Welch, 1965). Métodos como de (Viecelli, 1969), (Hirt e Nichols, 1971), (Hirt e Cook, 1972), (Miyata e Masuko, 1985), (Tomé e McKee, 1994) e outros são exemplos 
de técnicas numéricas baseadas no método MAC. Neste trabalho, o objeto de estudo é a metodologia GENSMAC. Essa metodologia utiliza a técnica de variáveis primitivas na formulação pressão-velocidade e é baseado no método de projeção de Chorin (Chórin, 1967). A potencialidade do método GENSMAC foi reconhecida na literatura em DFC (Griebel et al., 1998), (Dartzi et al., 1998), desde a primeira publicação de (Tomé e McKee, 1994). Porém, escoamentos a altos valores do número de Reynolds ofereciam sérias complicações para essa metodologia. Desta forma, (Ferreira et al., 2002) introduziram modificações na metodologia GENSMAC com o objetivo de resolver problemas de escoamentos com superfícies livres a altos números de Reynolds.

Atualmente, uma dificuldade na metodologia GENSMAC é sua formulação explícita, pois geralmente, a aplicação de métodos explícitos, requer um número elevado de ciclos computacionais e, como resultado, pode consumir grande quantidade de memória, tempo de processamento elevado e bastante espaço de armazenamento. Esses problemas ocorrem, freqüentemente, em simulações onde o valor do número de Reynolds é baixo $(R e<<1)$ ou quando o refinamento da malha é necessário para se obter uma solução numérica melhor, pois o passo temporal $\delta t$ se torna muito pequeno. $\mathrm{O}$ valor de $\delta t$ se torna muito restrito devido à condição de estabilidade imposta pelos métodos explícitos, que envolvem o número de Reynolds e o espaçamento da malha. Uma alternativa para resolver esse inconveniente é aplicar formulações implícitas.

Uma motivação para o uso de métodos implícitos para as equações de Navier-Stokes é explorar a favorável propriedade de estabilidade. O uso dos métodos implícitos para as equações diferenciais parciais iniciou-se em 1947, quando Crank e Nicolson (Crank e Nicolson, 1947) empregaram para a equação da difusão um método incondicionalmente estável. Logo após, em 1955 Peaceman e Rachford (Peaceman e Rachford, 1955) e (Douglas e Rachford, 1960) introduziram os primeiros métodos ADI (Alternating Direction Implicit). Baseados nesses métodos, alguns autores como (Douglas e Kim, 2001) propuseram modificações em algumas dessas formulações implícitas. Discussões sobre as propriedades das formulações implícitas podem ser encontradas em (Lapidus e Pinder, 1982), (Sod, 1989), (Chapra e Canale, 1990), (Thomas, 1995), (Cuminato e Meneguette, 1999), (Cunha, 2000), (Fortuna, 2000) e outros.

A formulação implícita em muitos problemas da DFC é a preferida, pela possibilidade de avançar no tempo com $\delta t$ maiores. Entretanto, usar a formulação implícita não significa dizer que pode-se usar qualquer tamanho do passo temporal, pois quando resolve-se mais de uma equação, o problema do acoplamento pode limitar severamente 
o $\delta t$. Outra limitação que pode surgir é em problemas com superfícies livres, pois esse tipo de contorno requer uma atenção especial em seu tratamento. Portanto, estudos do refinamento de $\delta t$, para se determinar uma solução independente de $\delta t$ num dado nível de tempo, devem sempre ser conduzidos. Deve-se lembrar que a formulação implícita dá origem a um sistema linear de equações. Em muitos problemas de escoamentos de fluidos, esses sistemas são esparsos podendo ou não serem simétricos. Desta forma, outro cuidado na aplicação de formulações implícitas é a escolha de métodos apropriados para a resolução de sistemas lineares. Mais detalhes sobre métodos para sistemas lineares podem ser encontrados em (Lanczos, 1952), (Watson, 1976), (Luenberger, 1984), (Golub e Loan, 1989), (Vorst, 1992), (Press et al., 1992), (Cuminato e Meneguette, 1999), (Cunha, 2000), (Ayachour, 2003) e outros.

Autores como (Roache, 1998) e (Briley e McDonald, 2001) apresentam, em seus trabalhos, um resumo de vários métodos que utilizam formulações implícitas para resolver as equações de Navier-Stokes. Alguns exemplos, como os métodos apresentados por (Pearson, 1965), (Briley, 1971) e outros introduziram formulações implícitas utilizando a metodologia corrente-pressão (Roache, 1998). Porém, para problemas tridimensionais essa metodologia não se aplica facilmente. Desta forma, a formulação pressão-velocidade (Roache, 1998) é a mais adequada para escoamentos de fluidos. Autores como (Harlow e Welch, 1965), (Chorin, 1967), (Chorin, 1968), (Amsden e Harlow, 1970) foram os precursores no desenvolvimento de métodos para tratar o acoplamento pressão-velocidade. Baseados na formulação pressão-velocidade, (Briley e McDonald, 1975) e (Briley e McDonald, 1977) apresentaram métodos que utilizam formulações implícitas para fluidos compressíveis. No caso de fluidos incompressiveis, alguns dos trabalhos mais conhecidos que utilizam formulações implícitas são: (Pracht, 1971), (Caretto et al., 1972), (Deville, 1975). (Turek, 1996), (Armenio, 1997), (Bansch, 1998), (Manna e Vacca. 1999), (Vuik et al., 2000), (Langtangen e Winther, 2002), (Bijl et al., 2002), (Street, 2003) e outros.

Reconhecendo o interesse considerável em soluções numéricas representativas para escoamentos incompressíveis com superfícies livres a baixo número de Reynolds, este trabalho tem como objetivos principais analisar e implementar formulações implícitas. Em busca de tais objetivos, as formulações Implícitas Regressivas e Crank-Nicolson, baseados em variações dos métodos de Projeção (Kim e Moin, 1985) e (Gresho, 1990) foram analisadas e adaptadas à metodologia GENSMAC. Como conseqüência, este trabalho apresenta uma série de resultados numéricos de escoamentos de fluidos incom- 
pressíveis, mostrando a capacidade atual da metodologia GENSMAC de abordar problemas bidimensionais com superfícies livres para valores baixos do número de Reynolds.

O presente trabalho está organizado da seguinte maneira:

- No capítulo 2 apresenta-se uma breve discussão geral das propriedades de métodos numéricos para equações diferenciais;

- No capítulo 3 a formulação matemática e as equações de Navier-Stokes são derivadas;

- No capítulo 4 o método de Projeção e suas variações são apresentados juntamente com uma discussão das formulações implícitas para as equação de Navier-Stokes;

- No capítulo 5 apresenta-se a metodologia GENSMAC e a aplicação das formulações implícitas nessa metodologia com as devidas modificações;

- No capítulo 6 as equações utilizadas são discretizadas e o ambiente de simulação FreeFlow2d é apresentado;

- No capítulo 7 resultados numéricos da aplicação das formulações implícitas apresentadas no capítulo 4 são discutidos;

- O capítulo 8, finalmente, contêm uma síntese do presente trabalho, algumas conclusões e sugestões para trabalhos futuros. 


\section{Métodos Numéricos para Equações Diferenciais: Conceitos Básicos}

\subsection{Introdução}

A modelagem matemática de problemas reais em ciências aplicadas vem a cada dia se tornado uma ferramenta mais útil na compreensão dos fenómenos envolvidos nesses problemas. A modelagem matemática permite a simulação computacional desses problemas e vem revelando-se determinante no desenvolvimento de novas tecnologias. O resultado da modelagem matemática de um problema, é um conjunto de equações que deve ser resolvido em computadores, produzindo uma solução numérica, denominada simulação e descreve propriedades do problema em questão.

A simulação numérica pode ser vista como uma relação entre resultados teóricos e práticos, ou seja, sendo uma nova solução para determinados problemas, despertando o interesse de muitos pesquisadores. A simulação numérica, muitas vezes não apresenta as restrições que resultados teóricos e práticos podem impor na modelagem da física do problema. Desta forma, para que a solução numérica seja aplicada a um problema, expressões matemáticas devem ser derivadas. Essas expressões matemáticas, geralmente são definidas a partir da aplicação de princípios físicos, descritos por leis e princípios adequadas ao fenômeno, como conservação de massa, energia e movimento. Os modelos 
8 Capítulo 2-Métodos Numéricos para Equações Diferenciais: Conceitos Básicos

resultantes são constituído de equações que relacionam grandezas e propriedades entre si.

Neste trabalho, a atenção é voltada para a modelação de problemas que envolvem escoamentos de fluidos. A solução desses problemas requer o manuseio das equações de Navier-Stokes. Desta forma, para se obter simulações computacionais confiáveis é necessário um estudo sobre o tratamento numérico das equações de conservação, que são expressas por Equações Diferenciais Parciais (EDP). Logo, o estudo das propriedades numéricas das EDPs, torna-se o primeiro passo para se obter bons resultados nas simulações numéricas de escoamentos de fluidos. O estudo dessas propriedades numéricas pode seguir o esquema abaixo:

1. Selecionar o método de discretização das equações de interesse. Isto implica selecionar entre os métodos de Diferenças Finitas, Volumes Finitos ou Elementos Finitos. No presente trabalho, o método de discretização utilizado é o método de Diferenças Finitas sobre uma malha estruturada;

2. Selecionar as fórmulas de discretização, como diferenças avançadas (ou progressivas), diferenças atrasadas (ou regressivas), diferenças centradas e outras;

3. Escolher a técnica de resolução das equações: Método Explícito, Implícito ou Semi-Implícito;

4. Análise numérica do método escolhido. O trabalho nesse passo concentra-se em analisar a consistência, estabilidade e convergência do método numérico.

Detalhes das propriedades de métodos numéricos para EDPs podem ser encontrados em (Lapidus e Pinder, 1982), (Sod, 1989), (Chapra e Canale, 1990), (Thomas, 1995), (Cuminato e Meneguette, 1999), (Cunha, 2000), (Fortuna, 2000) e outros. Neste capítulo, uma breve introdução aos principais conceitos e definições de propriedades dos métodos numéricos para EDPs, fundamentais no presente trabalho, será apresentada.

\subsection{Aproximação por Diferenças Finitas}

O método de diferenças finitas é baseado na expansão em série de Taylor de uma função. Este método foi primeiramente empregado por Euler em 1768. A idéia do 
método de diferenças finitas é substituir as derivadas numa Equação Diferencial (ED) por aproximações, construindo assim, uma Equação de Diferenças Finitas (EDF).

Para a aplicação do método de diferenças finitas, considere como exemplo, no caso bidimensional, uma EDP envolvendo o tempo $t$, duas coordenadas espaciais $x$ e $y$ e uma função $f=f(x, y, t)$. Na EDF, que aproxima uma EDP, os índices $i$ e $j$ são utilizados para representar as coordenadas cartesianas $x$ e $y$, e o índice $n$ é usado para representar o tempo.

Para se obter a solução numérica de uma EDP, definida sobre um domínio $\Omega$, é necessário, tratar $\Omega$ de forma discreta, ou seja, não é possivel tratar o domínio como uma região contínua, pois a solução numérica é obtida em pontos discretos $\left(x_{i}, y_{j}, t_{n}\right)$. Portanto, para utilizar o método de diferenças finitas, escolhe-se um conjunto de pontos em $\Omega$ para se obter a solução numérica. A escolha desses pontos define a discretização do domínio, e o conjunto desses pontos define a malha.

Neste trabalho, adota-se a filosofia de malhas estruturadas, definindo assim, uma malha retangular paralela aos eixos, com espaçamento $\delta x$ na direção $x$ e $\delta y$ na direção $y$ : e na discretização temporal com passo $\delta t$. A seguir, alguns exemplos de aproximações de derivadas por diferenças finitas, para uma função $f(x, y)$.

- Diferenças Avançadas (ou Progressivas)

$$
\frac{\partial f\left(x_{i}, y_{j}\right)}{\partial x} \simeq \frac{f_{i+1, j}-f_{i, j}}{\partial x}-\frac{(\delta x)}{2} \frac{\partial^{2} f(\xi, y)}{\hat{\sigma} x^{2}}, \quad \xi \in\left(x_{i}, x_{i+1}\right)
$$

onde o último termo da expressão (2.1) representa o erro da aproximação. Essa notaçã̃o será utilizada para as demais aproximações.

$$
\frac{\partial f\left(x_{i}, y_{j}\right)}{\partial y} \simeq \frac{f_{i, j+1}-f_{i, j}}{\delta y}-\frac{(\delta y)}{2} \frac{\partial^{2} f(x, \zeta)}{\partial y^{2}}, \quad \zeta \in\left(y_{j}, y_{j+1}\right)
$$

- Diferenças Atrasadas (ou Regressivas)

$$
\begin{array}{ll}
\frac{\partial f\left(x_{i}, y_{j}\right)}{\partial x} \simeq \frac{f_{i, j}-f_{i-1, j}}{\delta x}+\frac{(\delta x)}{2} \frac{\partial^{2} f(\xi, y)}{\partial x^{2}}, & \xi \in\left(x_{i-1}, x_{i}\right) \\
\frac{\partial f\left(x_{i}, y_{j}\right)}{\partial y} \simeq \frac{f_{i, j}-f_{i, j-1}}{\delta y}+\frac{(\delta y)}{2} \frac{\partial^{2} f(x, \zeta)}{\partial y^{2}}, \quad & \zeta \in\left(y_{j-1}, y_{j}\right) .
\end{array}
$$


- Diferenças Centrais

$$
\begin{gathered}
\frac{\partial f\left(x_{i}, y_{j}\right)}{\partial x} \simeq \frac{f_{i+1, j}-f_{i-1, j}}{2 \delta x}-\frac{(\delta x)^{2}}{6} \frac{\partial^{3} f(\xi, y)}{\partial x^{3}}, \quad \xi \in\left(x_{i-1}, x_{i+1}\right) \\
\frac{\partial f\left(x_{i}, y_{j}\right)}{\partial y} \simeq \frac{f_{i, j+1}-f_{i, j-1}}{2 \delta y}-\frac{(\delta y)^{2}}{6} \frac{\partial^{3} f(x, \zeta)}{\partial y^{3}}, \quad \zeta \in\left(y_{j-1}, y_{j+1}\right) \\
\left.\frac{\partial^{2} f\left(x_{i}, y_{j}\right)}{\partial x^{2}} \simeq \frac{f_{i+1, j}-2 f_{i, j}+f_{i-1, j}}{\delta x^{2}}-\frac{(\delta x)^{2}}{12} \frac{\partial^{4} f(\xi, y)}{\left.\partial x^{4}\right)}\right), \quad \xi \in\left(x_{i-1}, x_{i+1}\right) \\
\left.\frac{\partial^{2} f\left(x_{i}, y_{j}\right)}{\partial y^{2}}=\frac{f_{i, j+1}-2 f_{i, j}+f_{i, j-1}}{\delta y^{2}}-\frac{(\delta y)^{2}}{12} \frac{\partial^{4} f(x, \zeta)}{\partial y^{4}}\right), \quad \zeta \in\left(y_{j-1}, y_{j+1}\right) .
\end{gathered}
$$

\subsubsection{Aplicação do Método de Diferenças Finitas para EDPs}

Nesta subseção, os métodos explícito, implícitos e semi-implícitos serão apresentados para algumas EDPs. Em todos os exemplos, a derivada em relação ao tempo será aproximada por diferenças progressivas.

- Equação de difusão em duas dimensões

Considere a equação modelo

$$
\frac{\partial u}{\partial t}=u\left(\frac{\partial^{2} u}{\partial x^{2}}+\frac{\partial^{2} u}{\partial y^{2}}\right)
$$

onde $\alpha$ é uma constante. Utilizando diferentes fórmulas de diferenças finitas para aproximar as derivadas, obtêm-se:

- Método explícito

$$
U_{i, j}^{n+1}=U_{i, j}^{n}+\sigma_{x}\left(U_{i+1, j}^{n}-2 U_{i, j}^{n}+U_{i-1, j}^{n}\right)+\sigma_{y}\left(U_{i, j+1}^{n}-2 U_{i, j}^{n}+\bar{U}_{i, j-1}^{\prime \prime} j\right)
$$


- Método implícito regressivo

$$
U_{i, j}^{n+1}-\sigma_{x}\left(U_{i+1, j}^{n+1}-2 U_{i, j}^{n+i}+U_{i-1, j}^{n+1}\right)-\sigma_{y}\left(U_{i, j+1}^{n+1}-2 U_{i, j}^{n+1}+U_{i, j-1}^{n+1}\right)=U_{i, j}^{n},
$$

- Método de Crank-Nicolson

$$
\begin{aligned}
& U_{i, j}^{i+1}-\frac{\sigma_{x}}{2}\left(U_{i+1, j}^{n+1}-2 U_{i, j}^{n+1}+U_{i-1, j}^{n+i}\right)-\frac{\sigma_{y}}{2}\left(U_{i, j+1}^{n+1}-2 U_{i, j}^{n+1}+U_{i, j-1}^{n+1}\right)= \\
& U_{i, j}^{n}+\frac{\sigma_{x}}{2}\left(U_{i+1, j}^{n}-2 U_{i, j}^{n}+U_{i-1, j}^{n}\right)+\frac{\sigma_{y}}{2}\left(U_{i, j+1}^{n}-2 U_{i, j}^{n}+U_{i, j-1, j}^{n}\right) .
\end{aligned}
$$

Define-se os números de difusão (Roache, 1998) como

$$
\sigma_{x}=\frac{\alpha \delta t}{(\delta x)^{2}} \quad \text { e } \quad \sigma_{y}=\frac{\alpha \delta t}{(\delta y)^{2}}
$$

- Equação de convecção em duas dimensões

Considere a equação modelo

$$
\frac{\partial u}{\partial t}-u\left(\frac{\partial u}{\partial x}\right)+v\left(\frac{\partial u}{\partial y}\right)
$$

com $a>0$ e $b>0$. Utilizando diferenças progressivas para a derivada temporal e diferenças centrais para as espaciais, obtêm-se:

- Método explícito

$$
U_{i, j}^{n+1}=\frac{\eta_{x}}{2}\left(U_{i+1, j}^{n}-U_{i-1, j}^{n}\right)+\frac{\eta_{y}}{2}\left(U_{i, j+1}^{n}-U_{i, j-1}^{n}\right)
$$

Define-se os números de Courant como

$$
\eta_{x}=\frac{a \delta t}{\hat{\delta x}} \quad \text { e } \quad \eta_{y}=\frac{b \delta t}{\delta y}
$$

- Equação da convecção-difusão em duas dimensões

Considere a equação modelo

$$
\frac{\partial u}{\partial t}--\hat{u}\left(\frac{\partial u}{\partial x}\right)-z\left(\frac{\partial u}{\partial y}\right) ; \hat{u}\left(\frac{\partial^{2} u}{\partial x^{2}}+\frac{\partial^{2} u}{\partial y^{2}}\right) .
$$


12 Capítulo 2-Métodos Numéricos para Equações Diferenciais: Conceitos Básicos

Os termos difusivos

$$
\alpha\left(\frac{\partial^{2} u}{\partial x^{2}}+\frac{\partial^{2} u}{\partial y^{2}}\right)
$$

serão discretizado no nível de tempo $n+1$ utilizando diferenças centrais de segunda ordem, e os termos convectivos

$$
-a\left(\frac{\partial u}{\partial x}\right)-b\left(\frac{\partial u}{\partial y}\right)
$$

serão discretizado no nível de tempo $n$ utilizando diferenças centrais de segunda ordem. Portanto o método utilizado para aproximar a equação (2.17) é definido como semi-implícito. A equação (2.17) é escrita na forma:

$$
\begin{aligned}
& U_{i, j}^{2+1}-\sigma_{x}\left(U_{i+1, j}^{n-1}-2 U_{i, j}^{n+1}+U_{i-1, j}^{n+1}\right)-\sigma_{y}\left(U_{i, j+1}^{n+1}-2 U_{i, j}^{n+1}+U_{i, j-1}^{n+1}\right)= \\
& -\frac{\eta_{x}}{2}\left(U_{i+1, j}^{n}-U_{i-1, j}^{n}\right) x-\frac{\eta_{y}}{2}\left(U_{i, j+1}^{n}-U_{i, j-1}^{n}\right) .
\end{aligned}
$$

\subsection{Consistência}

Um requisito importante de uma aproximação de diferenças finitas é que ela seja consistente com a equação diferencial parcial que discretiza. Quando as derivadas de uma EDP são substituídas por diferenças, uma equação de diferenças finitas (EDF) é produzida e um erro introduzido, de forma que a solução exata da EDP não satisfaz a EDF precisamente. Esse erro é definido como erro de truncamento local (ETL) e denotado por $\tau_{i, j}^{\tau}$. No entanto, no limite quando o espaçamento da malha $\delta x, \delta y \mathrm{e}$ o temporal $\delta t$ tendem para zero deseja-se que a EDF esteja próxima da EDP. Esta é a condição para a consistência que será formalmente definida por: um método é consistente se o erro de truncamento local tender a zero para $\delta x$, $\delta y$ e $\delta t$ tendendo a zero. Como aplicação da análise do ETL, considere o método explícito para a equação da difusão (2.9), escrito na forma

$$
\frac{U_{i, j}^{n+1}-U_{i, j}^{n}}{\delta t}=\alpha\left(\frac{I J_{i+1, j}^{n}-2 U_{i, j}^{n}+I_{i-1, j}^{n}}{\delta x^{2}}+\frac{U_{i, j+1}^{n}-2 U_{i, j}^{n}+U_{i, j-1}^{n}}{\delta y^{2}}\right) .
$$

Os valores $U_{i, i}^{n}$ na equação (2.21) são soluções numéricas e denota-se por $u_{i, j}^{n}=$ $u(i \delta x, j \delta y, n \delta t)$ a solução exata da equação diferencial, onde $u(x, y, t)$ é a solução analítica. 
Substituindo a solução exata no ponto $\left(x_{i}, y_{j}, t_{n}\right)$ na equação de diferenças $(2.21)$, tem-se

$$
\frac{u_{i, j}^{n+1}-u_{i, j}^{n}}{\delta t}-\alpha\left(\frac{u_{i+1, j}^{n}-2 u_{i, j}^{n}+u_{i-1, j}^{n}}{\delta x^{2}}+\frac{u_{i, j+1}^{n}-2 u_{i, j}^{n}+u_{i, j-1}^{n}}{\delta y^{2}}\right)=-\tau_{i, j}^{n},
$$

mostrando que os valores exatos $\overline{u_{i, j}}$ não satisfazem a equação de diferenças, mas são soluções de uma equação discretizada modificada com erro de truncamento local no lado direito. Portanto o ETL é uma medida de quanto a solução da equação diferencial, calculada nos pontos da malha, deixa de satisfazer a equação de diferenças. Se a função $u(x, y, t)$ é suficientemente suave pode-se utilizar a expansão em série de Taylor em torno do ponto $\left(x_{i}, y_{j}, t_{n}\right)$ para os termos da equação de diferenças (2.22) que não estejam avaliados nesse ponto. Substituindo essas expansões na equação (2.22) e utilizando a equação diferencial (2.9), escreve-se o erro de truncamento local como:

$$
\tau_{i, j}^{n}=\frac{\delta t}{2} \frac{\partial^{2} u}{\partial t^{2}}-\frac{\delta x^{2}}{12} \frac{\partial^{4} u}{\partial x^{4}}-\frac{\delta y^{2}}{12} \frac{\partial^{4} u}{\partial y^{4}}+O\left(\delta t^{2}\right)+O\left(\delta x^{3}\right)+O\left(\delta y^{3}\right)
$$

onde a notação $O$ representa a ordem do erro das aproximações ao utilizar a expansão em série de Taylor.

A ordem de precisão de um método é também definida pelo ETL. Se o ETL é escrito na forma

$$
\tau_{i, j}^{n}=O\left(\delta t^{p}\right)+O\left(\delta x^{q}\right)+O\left(\delta y^{\tau}\right)
$$

onde $p, q$ e $r$ são os menores expoentes na expressão do ETL relacionados a $\partial t, \delta x$ e $\delta y$ respectivamente, o método é dito ser de ordem $p$ no tempo e ordens $q$ e $r$ no espaço.

Portanto, para a equação da difusão (2.9) tem-se que

- Método explícito é de ordem $O(\delta t)+O\left(\delta x^{2}\right)+O\left(\delta y^{2}\right)$;

- Método implícito regressivo é de ordem $O(\delta t)+O\left(\delta x^{2}\right)+O\left(\delta y^{2}\right)$;

- Método Crank-Nicolson é de ordem $O\left(\delta t^{2}\right)+O\left(\delta x^{2}\right)+O\left(\delta y^{2}\right)$.

\subsection{Estabilidade}

O conceito mais geral de estabilidade foi introduzido por (Lax e Richtmyer, 1956) e logo após empregado por diversos autores, como (Richtmyer e Morton, 1967). Esse 
14 Capítulo 2-Métodos Numéricos para Equações Diferenciais: Conceitos Básicos conceito, diz que.se um método numérico é estâvel, então quaisquer erros ou perturbações na solução não são amplificadas sem limite, ou seja, o conceito de estabilidade está relacionado ao crescimento ou decaimento dos erros introduzidos nos cálculos. No caso em que o problema analisado é do tipo transiente, o conceito de estabilidade pode ser visto com uma garantia que a solução numérica é limitada.

Uma maneira prática de se evitar a propagação de erros durante os cálculos, é aplicar métodos ou critérios de estabilidade, verificando se o método é ou não estável. Os métodos numéricos podem ser classificados como:

1. Condicionalmente estáveis: São métodos que devem satisfazer uma condição de estabilidade para que suas soluções numéricas sejam estáveis;

2. Incondicionalmente estáveis: São métodos que não precisam satisfazer critérios de estabilidades para que suas soluções numéricas sejam estáveis;

3. Incondicionalmente instáveis: Não há critério para que suas soluções sejam estáveis.

Existem vários critérios para o estudo da estabilidade de um método numérico: método de perturbação discreta descrito por (Thoman e Szewczyk, 1966), critério de Von Neumann descrito por (Charney et al., 1950), método de Hirt ou método da equação modificada melhorada descrito por (Hirt, 1968), critério da matriz descrito por (Eddy, 1949), e outros.

Nesta seção os critérios de Von Neumann e da matriz serão brevemente apresentados. Exemplos da aplicação dos métodos de perturbação discreta, equação modificada melhorada e outros podem ser encontrados em (Hoffmann e Chiang, 1993), (Roache, 1998) e (Fortuna, 2000).

\subsubsection{Critério de Von Neumann}

O critério de Von Neumann é um procedimento muito aplicado para estudar a estabilidade de uma equação de diferenças finitas. Neste método, a solução da equação de diferenças finitas é expandido em série de Fourier. O decaimento ou crescimento do fator de amplificação indica se o método é ou não estável.

O critério de Von Neumann pode ser utilizado quando o efeito das condições de contorno na estabilidade da solução podem ser ignoradas ou removidas. Esse critério 
é importante para decidir, na prática, a estabilidade de um método numérico, pois ele é de fácil aplicação e é uma condição necessária para estabilidade. Em alguns casos é também uma condição suficiente.

Suponha que o erro de um método numérico possa ser expandido em série de Fourier da seguinte maneira:

$$
\varepsilon_{i}^{n}=\sum_{j=0}^{N} E_{j}^{n} e^{I k_{j} i \delta x}, \quad i=0,1 . . N
$$

onde $k_{j}=\frac{2 j \pi}{L}$ é o número de onda na direção $x, L$ é o comprimento na direção $x, E_{j}^{n}$ é a amplitude no nível de tempo $n, I=\sqrt{-\overline{1}}$ e $i \delta x=x_{i}$, onde $i$ é o índice da malha e $\delta x=\frac{\bar{L}}{i_{i}}$. Detalhes sobre a decomposição de Fourier para o erro podem ser visto em (Lapidus e Pinder, 1982), (Hirsch, 1989), (Hoffmann e Chiang, 1993), (Thomas, 1995) e (Roache, 1998). O produto $k_{j} \delta x$ é representado como um parâmetro de fase

$$
\theta_{x} \equiv k_{j} \delta x, \quad \text { com } 0 \leq \theta_{x} \leq 2 \pi
$$

Na prática, quando deseja-se aplicar o critério de Von Neumann verifica-se como a equação de diferenças amplifica um harmônico simples da forma

$$
U_{i}^{n}=E^{n} e^{I \theta_{x} i}
$$

e analogamente,

$$
\begin{aligned}
U_{i}^{n+1} & =E^{n+1} e^{I \theta_{x} i} \\
U_{i \pm 1}^{n} & =E^{n} e^{I \theta_{x}(i \pm 1)}
\end{aligned}
$$

A condição de estabilidade para uma equação de diferenças finitas será satisfeita se o fator $E^{n}$ não crescer com o tempo, isto é, se a razão

$$
\left|\frac{E^{n+1}}{E^{n}}\right| \leq 1
$$

para todo $\theta_{x}$. A quantidade $G$, definida por

$$
G=\frac{E^{n+1}}{E^{n}}
$$

é o fator de amplificação. 


\section{Capítulo 2 - Métodos Numéricos para Equações Diferenciais: Conceitos Básicos}

O critério de Von Neumann pode ser facilmente estendido para problemas bidimensionais, ou seja, a componente de Fourier (2.25), agora assume a seguinte forma

$$
U_{-, j}^{n}=E^{n} e^{I\left(\theta_{x} i+\theta_{y} j\right)}
$$

Diversos modelos matemáticos que descrevem problemas de escoamentos de fluidos contêm termos não-lineares, ou eventualmente, coeficientes não constantes. Nestes casos, o critério de Von Neumann não pode ser rigorosamente aplicado. A discussão completa sobre esse assunto pode ser encontrada em (Richtmyer e Morton, 1967), (Hirsch, 1989) e (Roache, 1998).

Em resumo, para a aplicação do critério de Von Neumann, algumas considerações devem ser observadas:

1. A influência das condições de contorno na estabilidade da solução não é incluída. O método de Von Neumann é baseado na existência da decomposição de Fourier da solução no domínio computacional no espaço. Isto implica que a presença de condições de contorno não periódicas não é analisada. Uma solução para este problema, é utilizar o método da matriz, descrito na próxima seção.

2. Um método é estável se $|G| \leq 1$.

3. Este procedimento pode ser usado para analisar a estabilidade de um sistema de EDFs. A condição a ser imposta então será sobre os autovalores da matriz de amplificação, obtidos pela solução de $P(\lambda)=\operatorname{det}|G-\lambda I|=0$. Isto pode ser encontrado em (Casulli, 1981), (Mansour e Hamed, 1990), (Casulli, 1990) e (Petersson, 2001).

4. Na ocasião onde o fator de amplificação é uma expressão difícil de se analisar, uma solução gráfica ou alguns experimentos numéricos podem facilitar a análise (ver (Hirsch, 1989), (Hoffmann e Chiang, 1993) e (Roache, 1998)).

5. Para problemas não-lineares, a estabilidade da equação linearizada é uma condição necessária. O leitor interessado em problemas não-lineares ver (Briggs et al., 1983), (Mcdonough e Bywater, 1986), (Hirsch, 1989) e (LeVeque, 1990).

Portanto, aplicando o critério de estabilidade de Von Neumann, para os métodos explícito, implícito regressivo e Crank-Nicolson na equação da difusão (2.9), tem-se: 
- Método explícito é condicionalmente estável se $\left(\sigma_{x}+\sigma_{y} \leq \frac{1}{2}\right)$;

- Método implícito regressivo é incondicionalmente estável;

- Método Crank-Nicolson é incondicionalmente estável.

\subsubsection{Critério da Matriz}

O critério de Von Neumann não leva em consideração o efeito de condições de contorno não periódicas na análise de estabilidade. Já o critério da matriz, analisa esses efeitos, mas em muitas aplicações não é fácil calcular na prática a condição de estabilidade.

Considere o seguinte problema sobre o domínio $\Omega$ com contorno $\mathrm{I}$

$$
\frac{\partial u}{\partial t}=W(u)
$$

com

$$
\begin{aligned}
& u(\mathbf{x}, 0)=f(\mathbf{x}) \text { para } t=0 \text { e } \quad \mathbf{x} \in \Omega \\
& u(\mathbf{x}, t)=g(\mathbf{x}, t) \text { para } t>0 \text { e } \mathbf{x} \in \Gamma
\end{aligned}
$$

onde $\mathrm{W}$ é definido como um operador.

Esse problema pode ser transformado, depois da discretização do operador diferencial espacial, num sistema de equações diferenciais ordinárias no tempo

$$
\frac{d U}{d t}=S U+Q
$$

onde $U$ é o vetor dos valores de $u_{i}$ na malha, $Q$ contém termos não-homogêneos e valores das condições de contorno e $S$ é a matriz dos coeficientes.

A análise de estabilidade da discretização espacial é baseada nos autovalores da matriz $S$. Quando os autovalores $\lambda$ da matriz $S$ tem a parte real não-positiva, o método é considerável estável. Mais detalhes em (Siemieniuch e Gladwell, 1978), (Hirsch, 1989), (Roache, 1998) e (Cuminato e Meneguette, 1999). 


\section{$2.5 \quad$ A-Estabilidade}

O conceito de A-estabilidade foi introduzido por Dahlquist em 1963 para métodos lineares no contexto de Equações Diferenciais Ordinárias (EDO). Neste trabalho, uma breve descrição do conceito de A-estabilidade será introduzida. Para isto considere a EDO

$$
\frac{\mathrm{dy}}{\mathrm{dt}}=f(t, y(t))
$$

com $0 \leq t \leq T$ e $y(0)=y_{0}$. Como aplicação do conceito de A-estabilidade considere a fôrmula de Runge-Kutta (Lambert, 1973) aplicada em (2.33) obtendo umá solução numérica da forma

$$
y_{n+1}=y_{n}+\delta t \sum_{i=1}^{s} b_{i} k_{i}
$$

com

$$
k_{i}=f\left(t_{n}+c_{i} \partial t, y_{n}+\partial t \sum_{j=1}^{s} a_{i j} k_{j}\right), \quad(1 \leq i \leq s),
$$

onde $b_{i}, c_{i}$ e $a_{\imath j}$ são parâmetros reais.

Para definir o conceito de A-estabilidade, como exemplo considere uma equação escalar

$$
\frac{\text { dy }}{\mathrm{dt}}=\lambda y(t), \quad \lambda \in \mathbb{C}, \quad t \geq 0, \quad y(0)=y_{0}
$$

Embora essa equação seja simples ela será usada como modelo para a definição de A-estabilidade. Aplicando (2.34) em (2.36) obtêm-se uma solução numérica escrita da forma

$$
y_{n+1}=R(z) y_{n}
$$

onde $z=\lambda \delta t$ e $R(z): \mathbb{C} \rightarrow \mathbb{C}$ é um polinômio, de acordo com (Verwer e Dekker, 1984) escrito no forma

$$
R(z)=1+b^{T} z(I-A z)^{-1} e
$$

onde $A$ é uma matriz constante e $I$ a matriz identidade.

Define-se $R(z)$ como função de estabilidade. Portanto, o método é chamado Absolutamente estável em $z \in \mathbb{C}$, se, para este $z$,

$$
|R(z)| \leq 1
$$


Se o método é absolutamente estável para todo $z \in \mathbb{C}$ e $\operatorname{Re}(z) \leq 0$, então o método é chamado A-estável.

A região do plano complexo para qual $\left|R^{\prime}(z)\right| \leq 1$ é chamada região de estabilidade absoluta do método. Se a parte do plano complexo $C^{-}=\{z \in \mathbb{C}$ tal que $\operatorname{Re}(z) \leq 0\}$ está contida na região de estabilidade absoluta o método é dito A-estável.

O método é dito L-estável, se é A-estável e $\lim _{z \rightarrow \infty} R(z)=0$. Mais detalhes sobre métodos numéricos para EDO e conceitos de estabilidade podem ser encontrados em (Verwer e Dekker, 1984), (Hairer e Wanner, 1991), (Lambert, 1991), (Liotta et al., 2000) e (Larsson, 2002).

\subsubsection{Métodos A-estáveis}

Como foi discutido na subseção 2.4.2, o sistema obtido no processo de discretização é estável quando os autovalores $\lambda_{j}$ da matriz $S$ estão localizados no lado esquerdo do plano complexo.

Agora, considere um sistema na forma

$$
\frac{\mathrm{dU}}{\mathrm{dt}}=H(U, t)
$$

onde o vetor $\mathrm{H}(U, t)$ representa o operador de discretização espacial $S U+Q$ da equação (2.32). Aplicando um método linear de dois níveis ao sistema (2.40), obtêm-se o método dos trapézios generalizado, escrito na forma

$$
U^{n+1}-U^{n}=\delta t\left(\theta \mathrm{H}^{n+1}+(1-\theta) \mathrm{H}^{n}\right)
$$

De acordo com (Beam e Warming, 1982),

- Para $\theta=0$, obtêm-se o método de Euler explícito, que é de primeira ordem no tempo e não é A-estável;

- Para $\theta=1$, obtêm-se o método de Euler implícito, que é de primeira ordem no tempo e A-estável;

- Para $\theta=1 / 2$, obtêm-se o método de Crank-Nicolson, que é de segunda ordem no tempo e A-estável. 
O estudo da A-estabilidade desses métodos, pode ser feito da seguinte forma. Considere uma família particular de métodos de três níveis

$$
(1+\chi) U^{n+1}-(1+2 \chi) U^{n}=\delta t\left(\theta \mathrm{H}^{n+1}+(1-\theta) \mathrm{H}^{n}\right)-\chi U^{n-1}
$$

onde $\chi$ é um parâmetro livre.

Quando aplicado na equação de Navier-Stokes com diferenças centradas na variável espacial e com a linearização apropriada, esses métodos são conhecidos como Esquemas de Beam e Warming. O polinômio característico de (2.42), definido na seção 2.4, é escrito na forma:

$$
P(z)=(1+\chi) z^{2}-(1+2 \chi) z+\chi-(\lambda \delta t) z(\theta z+1-\theta)=0
$$

Para $\chi-0$ o método é de dois níveis e uma raíz de $P(z)$ é $z=0$ e a outra é

$$
z=\frac{1+(1-\theta) \lambda \delta t}{1-\theta \lambda \delta t}
$$

Para valores fixados de $\theta$ as regiões de estabilidade no plano complexo são obtidos pela condição de estabilidade

$$
|z| \leq 1
$$

Para determinar a região de estabilidade para valores específicos de $\theta$, considere que $\lambda \delta t$ seja da forma:

$$
\lambda \delta t=a+b I
$$

- Para $\theta=0$, tem-se

$$
z=1+\lambda \delta t
$$

ou $z=(1+a)+b I$. Segundo a condição de estabilidade (2.44), tem-se

$$
\sqrt{(1+a)^{2}+b^{2}} \leq 1 \Rightarrow(1+a)^{2}+b^{2} \leq 1
$$

$E$ isto implica que a região de estabilidade, conforme a figura 2.1, para o método explícito é o interior da circunferência de raio 1 e centro $(-1,0)$ do plano complexo $\lambda \delta t$. 


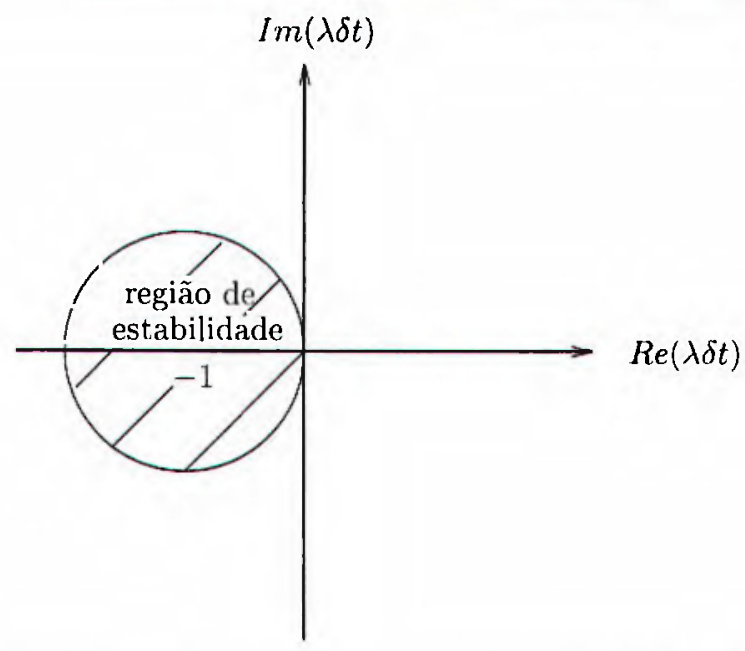

Figura 2.1: Região de estabilidade para $\theta=0$.

- Para $\theta=1$, tem-se

$$
z=\frac{1}{1-\lambda \delta t}-\frac{1}{(1-a)-b I}
$$

Utilizando a condição de estabilidade (2.44) obtêm-se

$$
1 \leq(1-a)^{2}+b^{2}
$$

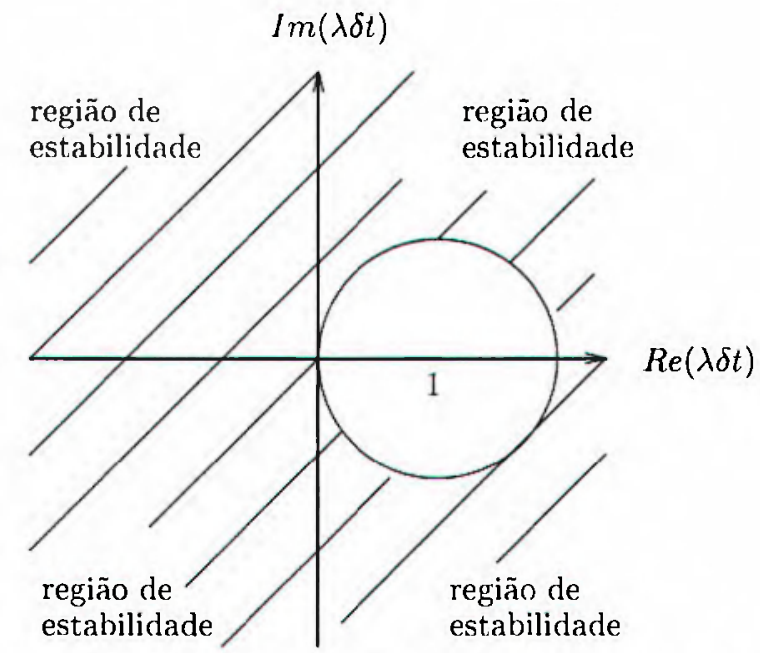

Figura 2.2: Região de estabilidade para $\theta=1$. 
Logo a região de estabilidade para o método implícito regressivo é a região externa à circunferência de raio 1 e centro $(1,0)$ do plano complexo $\lambda \delta t$, como mostra a figura 2.2 .

- $\operatorname{Para} \theta=1 / 2$,

$$
z=\frac{1+\frac{\lambda \delta t}{2}}{1-\frac{\lambda \delta t}{2}},
$$

e utilizando a condição de estabilidade (2.44) tem-se

$$
1+\frac{a}{2} \leq 1-\frac{a}{2} \Rightarrow a \leq 0
$$

Portanto a região de estabilidade do método de Crank-Nicolson é apresentada na figura 2.3, ou seja, a parte esquerda do plano complexo $\lambda \delta t$, incluindo o eixo imaginário.

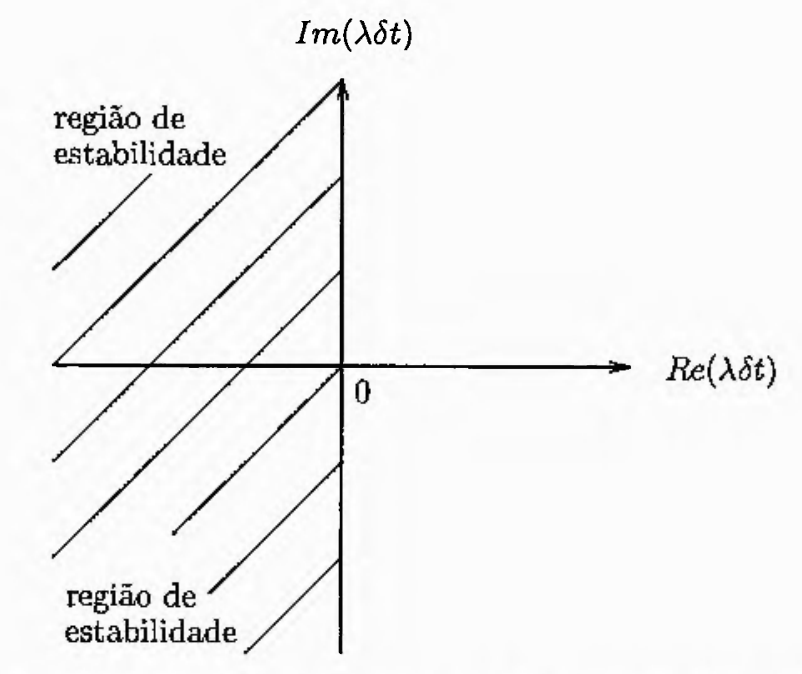

Figura 2.3: Região de estabilidade para $\theta=1 / 2$.

Note que para $\theta-1 / 2$ tem-se

$$
\lambda \delta t=2 \frac{z-1}{z+1}
$$

Portanto quando $\lambda \rightarrow \infty$ em (2.49), tem-se que uma raíz do polinômio característico do método Crank-Nicolson tende a -1 . Isto pode provocar oscilações nas soluções numéricas (Hirsch, 1989), quando $\delta t$ é grande. 


\subsection{Convergência}

A convergência de um método numérico é extremamente importante do ponto de vista prático, pois espera-se que a solução numérica calculada reproduza de maneira mais fiel possível a solução analítica do problema. Na seção 2.3, definiu-se como $U_{i, j}^{r}$ a aproximação numérica de $u(x, y, t)$, definida como solução analítica. Logo, a solução numérica deverá aproximar-se da solução analítica em cada ponto $(x, y, t)$ do domínio quando $\delta x, \delta y$ e $\delta t$ tendem para zero mantendo o ponto $(x, y, t)$ fixo, com $x=i \delta x$, $y=j \delta y$ e $t=n \delta t$, isto é, quando a malha é refinada. Esta é a condição para a convergência da solução numérica para solução a analítica da equação diferencial, que pode ser expressa pelo erro:

$$
\varepsilon_{i, j}^{n}=U_{i, j}^{n}-u(x, y, t),
$$

satisfazendo a seguinte condição de convergência

$$
\lim _{\delta x, \delta y, \delta i \rightarrow \hat{v}}\left|\varepsilon_{i, j}^{n}\right|=0
$$

Uma relação precisa entre as condições de consistência, estabilidade e convergência está contida no Teorema de Lax apresentado em (Richtmyer e Morton, 1967).

Teorema 1 Uma condição necessária e suficiente para a convergência de um método, quando aplicado a um problema linear de valor inicial bem posto é que o esquema de discretização seja consistente e estâvel.

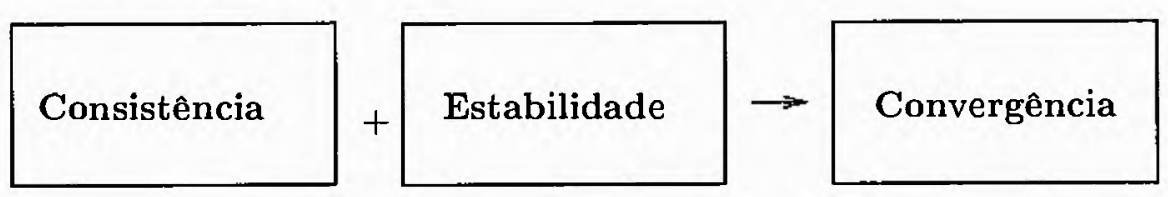

Figura 2.4: Teorema de Lax.

Ou seja, o teorema pode ser interpretado como um esquema prático (ver figura 2.4), e portanto, para o estudo de convergência de um método numérico deve-se:

1. Analisar a condição de consistência, pois isto implica na determinação da ordem de exatidão do esquema e o erro de truncamento local;

2. Analisar as propriedades de estabilidade, pois isto determina se o método é condicionalmente estável, incondicionalmente estável ou incondicionalmente instável. 
24 Capitulo 2 - Métodos Numéricos para Equações Diferenciais: Conceitos Básicos 


\section{Equações de Navier-Stokes: Derivação e Condições de Fronteira}

\subsection{Introdução}

Uma área de aplicação na qual as simulações numéricas desempenham um papel importante é na simulação de escoamentos de fluidos. No estudo dos escoamentos de fluidos incompressiveis, a modelagem matemática das leis de conservação é bem estabelecida pelas equações de conservação de massa e de Navier-Stokes. Atualmente, a simulação de escoamentos incompressíveis tem sido utilizada largamente na prática e com muito sucesso, graças ao desenvolvimento tecnológico. Uma classe de problemas em escoamentos que tem sido beneficiada com o avanço tecnológico é a dos problemas com superfícies livres em movimento.

Como mencionado anteriormente, para resolver problemas de escoamentos de fluidos é necessário definir o que é um fluido e classificar o tipo de escoamento. Um fluido pode ser definido como uma substância, que normalmente se encontra nos estados liquido ou gasoso, e que são incapazes de resistir às forças que causam tensões (essas forças são dadas pelo quociente entre o módulo de uma força e a área sobre qual ela age). Uma classificação do tipo de escoamento muito utilizada em DFC, é definir o escoamento como laminar ou turbulento. Para se entender o conceito de escoamentos laminares, basta observar o exemplo de uma torneira, aberta suavemente, onde a água escoa de 
maneira ordenada e bem comportada. Este é um exemplo clássico de escoamento laminar. Aumentando a vazão, o escoamento sofre transição para um regime desordenado e caótico, dando origem a um escoamento mais complexo, o escoamento turbulento. Neste trabalho, apenas os escoamentos laminares serão tratados.

A dinâmica de fluidos é a ciência que descreve o movimento de fluidos e estuda os fenómenos que envolvem fluidos em movimento. As leis da dinâmica dos fluidos são bem estabelecidas e podem ser formuladas por diversos caminhos. Geralmente, essas leis são conhecidas como leis de conservação, pois durante a evolução temporal de um escoamento, propriedades como massa, energia e movimento são conservadas. Essas leis expressam as leis da mecânica do contínuo.

O objetivo da dinâmica dos fluidos é determinar as propriedades de um fluido, esteja ele em movimento ou em repouso. Para isto, deve-se conhecer as variáveis que determinam o estado dos fluidos, as quais dependem em geral da posição no espaço e do tempo. Conseqüentemente, para conhecer o estado de um fluido, deve-se determinar o valor das variáveis que o identificam, ao longo do tempo em cada ponto do espaço ocupado pelo fluido. As variáveis que identificam o estado de um fluido incompressível e isotérmico são: a velocidade u em cada ponto (que é uma grandeza vetorial) e a pressão $p$ (que em cada ponto é uma grandeza escalar).

Essas variáveis são agrupadas em equações, que passaram a ser conhecidas em todo mundo como, as Equações de Navier-Stokes. Detalhes sobre as equações de Navier-Stokes, dinâmica dos fluidos, leis de conservação, mecânica do contínuo e outras propriedades de escoamento de fluidos podem ser encontrados em (Batchelor, 1970), (Peyret e Taylor, 1983), (Panton, 1984), (Flecther, 1992), (Maliska, 1995), (Anderson, 1995) e (Ferziger e Períc, 1999). Neste capítulo será apresentado as equações de interesse e algumas propriedades dos fluidos.

\subsection{Descrição do Movimento de Fluido}

As técnicas numéricas, nesse trabalho, tratam de problemas em mecânica do contínuo, e as propriedades físicas e mecânicas da matéria são descritas por meio de funções contínuas definidas no conjunto das coordenadas macroscópicas tempo e espaço. A descrição da cinemática do movimento de um fluido será brevemente discutida. 
O movimento de um fluido pode ser descrito por meio de uma transformação supondo que em um dado instante de tempo, uma determinada partícula esteja em uma posição $\mathrm{x}=\left(\overline{x_{1}}, \overline{x_{2}}\right)$, e num tempo posterior, a mesma partícula se encontre na posição $\mathbf{x}=\left(x_{1}, x_{2}\right)$. Admitindo que $\mathbf{x}$ é função de $t$ e da posição inicial $\overline{\mathbf{x}} \mathbf{e}$ considerando $o$ instante inicial como sendo o tempo $t=0$, tem-se

$$
\mathbf{x}=\mathbf{x}(\overline{\mathbf{x}}, t)
$$

para todo tempo $t$. As coordenadas $\overline{\mathrm{x}}$ são coordenadas materiais (ou lagrangeana) e as coordenadas $\mathrm{x}$ são coordenadas espaciais (ou euleriana). Assumindo que o movimento do fluido é uma função unívoca e contínua, pode-se inverter (3.1) para obter

$$
\overline{\mathbf{x}}=\mathbf{x}(\mathbf{x}, t)
$$

Do ponto de vista físico, o movimento contínuo do fluido pode ser expresso como: dada uma partícula no domínio do fluido, as partículas que estão na sua vizinhança permanecerão nesta vizinhança no transcorrer do movimento. A discussão completa da cinemática do movimento de um fluido pode ser encontrada em (Aris, 1962).

\subsection{Equações da Mecânica do Contínuo}

Como pode ser visto em (Panton, 1984), as equações da mecânica do contínuo podem ser derivadas do teorema do transporte de Reynolds (Aris, 1962). Essas equações que modelam o escoamento de fluidos, representam as expressões matemáticas de princípios físicos bem familiares, como

- Conservação de massa;

- Conservação da quantidade de movimento (segunda lei de Newton);

- Conservação de energia (primeira lei da termodinâmica).

Pode-se definir um fluido como um contínuo, onde as propriedades do escoamento são descritas em termos de grandezas macroscópicas, como pressão, velocidade e densidade (ou massa específica). Para o presente trabalho, as equações de interesse são: 


\section{- Conservação de massa}

O princípio de conservação de massa estabelece que na ausência de fontes de massa ou sumidouros de massa, toda a massa que entra em um sistema deve sair e/ou se acumular no sistema. Esse princípio pode ser entendido pelo diagrama da figura 3.1. O princípio de conservação de massa é expresso pela equação da continuidade:

$$
\frac{D \rho}{D t}+(\rho \nabla) \cdot \mathbf{u}=0
$$

onde

- $\frac{D}{D t}$ é a derivada total (ou substancial ou material), definida como:

$$
\frac{D \rho}{D t}=\frac{\partial \rho}{\partial t}+u-\frac{\partial \rho}{\partial x}+v \frac{\partial \rho}{\partial y}
$$

- $\nabla$ é o operador gradiente definido em coordenadas cartesianas bidimensionais por:

$$
\nabla=\mathrm{i} \frac{\partial}{\partial x}+\mathrm{i} \frac{\partial}{\partial y}
$$

com i e $\mathbf{j}$ os vetores unitários nas direções $x$ e $y$;

- $\mathbf{u}=(u, v)$ é o vetor velocidade;

- $\rho$ é a massa específica.

quantidade de massa acumulada no volume por unidade de tempo

$$
=-\begin{aligned}
& \begin{array}{l}
\text { Fluxo líquido de massa para fora } \\
\text { do volume }
\end{array} \\
& \text { a }
\end{aligned}
$$

Figura 3.1: Diagrama de Conservação de Massa.

\section{- Conservação de quantidade de movimento}

A equação de conservação de movimento é obtida aplicando-se a segunda lei de Newton a uma partícula de fluido. O diagrama apresentado pela figura 3.2, resume o princípio da conservação de movimento.

$\mathrm{Na}$ forma vetorial, a equação de conservação de movimento é dada por:

$$
\frac{D \rho \mathbf{u}}{D t}=\nabla \cdot \mathbf{T}+\rho \mathbf{g}
$$


onde

$$
\mathrm{T}=-p \mathrm{I}+\tau
$$

é o tensor de tensão total, que inclui o tensor tensão cisalhante $\tau$ e a pressão $p, \mathrm{I}$ é o tensor identidade e $\mathrm{g}$ é o vetor de aceleração gravitacional.

variação da quantidade de movimento no volume por unidade de tempo

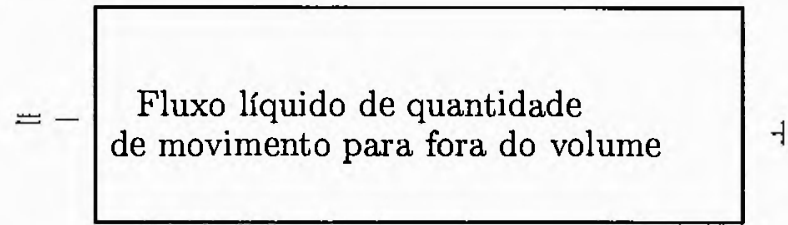

Resultante das forças aplicadas à superfície de contrôle

Figura 3.2: Diagrama de Conservação de Movimento.

\subsection{Fluidos Newtonianos}

Antes da definição de fluidos newtonianos é importante descrever a condição de incompressibilidade. Utilizando a hipótese de que a densidade $\rho$ do fluido não se altera no tempo, tem-se

$$
\frac{D \rho}{D t}=0
$$

e substituindo em (3.3), obtêm-se

$$
(\rho \nabla) \cdot \mathbf{u}=0
$$

e como $\rho \neq 0$, finalmente,

$$
\nabla \cdot \mathbf{u}=0
$$

A equação (3.5) é conhecida como condição de incompressibilidade, e será mantida em todo o domínio, isto é, todos os fluidos envolvidos na simulação obedecerão esta condição, e portanto, serão tomados como incompressíveis. 
Um fluido pode ser imaginado hipotéticamente como sendo formado por camadas que se movem umas relativamente às outras. Esse movimento produz uma tensão de cisalhamento entre as camadas. Como exemplo, considere o escoamento de um fluido, na direção $x$, sobre uma placa plana. A tensão de cisalhamento deforma um elemento de fluido em presença de um gradiente de pressão. Fisicamente, o conceito de deformação está associados à mudanças de forma de um corpo. A deformação por cisalhamento em um fluido ocorre, por exemplo, quando este está entre duas superfícies paralelas e uma delas desloca-se em relação à outra. Esse processo pode ser observado pela figura 3.3.
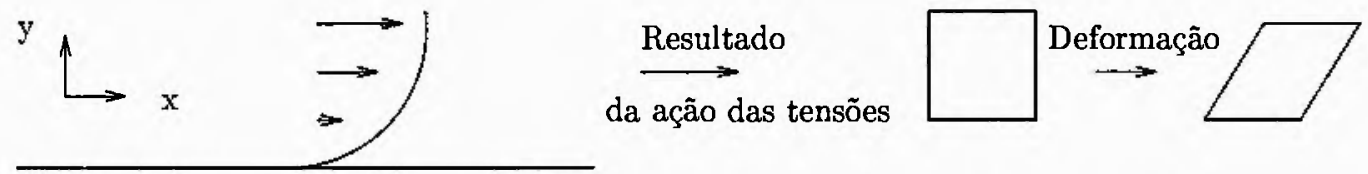

Figura 3.3: Exemplo da força de cisalhamento atuando sobre um fluido.

Define-se o tensor $\tau$ como

$$
\tau=\mu\left[(\nabla \mathbf{u})+(\nabla \mathbf{u})^{T}\right]
$$

onde $\mu$ é uma constante denominada coeficiente de viscosidade. Fluidos cuja tensão de cisalhamento é descrita por (3.6) oú fluidos que apresentam a relação tensão/taxa de deformação constante independente da deformação e do tempo denominam-se newtonianos (Batchelor, 1970), (Bretas e D'Avila, 2000). Os fluidos newtonianos são os mais freqüentes nos problemas de engenharia, embora existam outros tipos de fluidos cuja tensão de cisalhamento se exprime de outras formas em função do campo de velocidade, como os fluidos viscoelásticos e outros. Neste trabalho, apenas escoamento de fluidos newtonianos serão estudados.

Logo. a equação de conservação de quantidade de movimento na forma vetorial torna-se

$$
\frac{D(\rho \mathbf{u})}{D t}=\nabla \cdot\left(-p \mathbf{I}+\mu\left[(\nabla \mathbf{u})+(\nabla \mathbf{u})^{\mathrm{T}}\right]\right)+\rho \mathbf{g} .
$$

Agora utilizando a condição de incompressibilidade, tem-se

$$
\frac{D(\rho \mathbf{u})}{D t}=-\nabla p+\mu \nabla^{2} \mathbf{u}+\rho \mathbf{g}
$$


onde o produto $\rho$ g representa a ação do campo gravitacional. Neste trabalho, assume-se que a única força externa agindo sobre as partículas de fluido é a força gravitacional conservativa.

Considerando o fluido um meio homogêneo incompressível, a massa específica $\rho(\mathbf{x}, t)=$ $\rho_{0}$ das partículas não varia durante o seu movimento e as propriedades de transportes são constantes. Assim as equações (3.5) e (3.8) podem ser simplificadas para se obter na forma conservativa vetorial

$$
\begin{gathered}
\frac{\partial \mathbf{u}}{\partial t}+\nabla \cdot(\mathbf{u u})=-\nabla p+\nu \nabla^{2} \mathbf{u}+\mathbf{g}, \\
\nabla \cdot \mathbf{u}=0
\end{gathered}
$$

ou na forma tensorial

$$
\begin{aligned}
& \frac{\partial u_{i}}{\partial t}+\frac{\partial\left(u_{i} u_{j}\right)}{\partial x_{j}}=-\frac{\partial p}{\partial x_{i}}+\nu \frac{\partial}{\partial x_{j}}\left(\frac{\partial u_{i}}{\partial x_{j}}\right)+a_{i}, \quad i=1.2 \\
& \frac{\partial u_{i}}{\partial x_{j}}=0
\end{aligned}
$$

em que $\nu=\frac{\ddot{m}}{\mu_{0}}>0$ é o coeficiente de viscosidade cinemática molecular (constante) do fluido e $p$ a pressão cinemática $\left(p=\frac{p}{p_{0}}\right)$. As equações (3.9) e (3.10) constituem um sistema fechado de EDPs não-lineares e descrevem qualquer escoamento viscoso, incompressivel e isotérmico. Essas equações são conhecidas, respectivamente, como equações de Navier-stokes e equação da continuidade. Neste trabalho utiliza-se a formulação em variáveis primitivas (Dennis et al., 1979) para o tratamento numérico do conjunto de EDPs (3.9)-(3.10).

\subsection{Adimensionalização}

Os problemas em mecânica dos fluidos podem, em geral, ser caracterizados por grandezas específicas, como a velocidade do escoamento ou o diâmetro do tubo no qual ele escoa. Freqüentemente, trabalha-se com as variáveis da mecânica dos fluidos na forma adimensional, ou seja, grandezas dimensionais podem ser agrupadas em parâmetros adimensionais, que passam a caracterizar o escoamento, surgindo então alguns grupos adimensionais como o número de Reynolds, Strouhal e o de Froude. Através dessas grandezas, definem-se variáveis adimensionais segundo transformações lineares. 
A seguir são definidos alguns parâmetros importantes relacionados com as equações de Navier-Stokes.

- Número de Reynolds (Re): Representa a razão entre as forças inerciais (que são responsáveis pelo movimento do fluido) e as forças viscosas (que são responsáveis pela dissipação devido ao coeficiente de viscosidade molecular) do escoamento, dado por

$$
R e=\frac{\rho_{0} L U}{\mu_{0}}=\frac{\underline{I I}}{\nu_{0}}
$$

onde $\rho_{0}$ é a densidade, $L$ é a escala de comprimento, $U$ é a escala de velocidade, $\mu_{0}$ é o coeficiente de viscosidade e $\nu_{0}$ é o coeficiente de viscosidade cinemática (razão entre $\mu_{n}$ e $\rho_{0}$ ).

- Número de Strouhal (St): Representa a razão entre as escalas e uma freqüência $f_{r}$, ou seja

$$
S t=\frac{\underline{I} \cdot f}{V_{i}}
$$

- Número de Froude (Fr): Representa a razão entre as forças inerciais e as forças gravitacionais, isto é

$$
F r=\frac{U}{\sqrt{g L}}
$$

Neste trabalho, na adimensionalização das equações de conservação adota-se as transformações lineares conforme (Tomé e McKee, 1994), definindo as variáveis adimensionais, como segue:

$$
\rho=\rho_{0} \rho^{*} \quad \mu=\mu_{0} \mu^{*} \quad p=\rho_{0} U^{2} p^{*} \quad \mathbf{x}=L \mathbf{x}^{*} \quad \mathbf{u}=U \mathbf{u}^{*} \quad t=\frac{L}{Y} t^{*} \quad \mathbf{g}=g_{0} \mathbf{g}^{*}
$$

onde $\mathbf{x}=(x, y)$.

Substituindo as variáveis adimensionais nas equações de conservação de movimento e conservação de massa e eliminando "*” para simplificar, obtêm-se na forma adimensional

$$
\begin{aligned}
\frac{\partial \mathbf{u}}{\partial{ }^{\prime}}+\nabla \cdot(\mathbf{u u}) & =-\nabla p+\frac{\frac{1}{\bar{\kappa}} e}{} \nabla^{2} \mathbf{u}+\frac{1}{F r^{2}} \mathbf{g}, \\
& \nabla \cdot \mathbf{u}=0 .
\end{aligned}
$$

As equações (3.15) e (3.16) podem ser escritas na forma cartesiana em duas dimensões como 
- Conservação de massa ou Equação da continuidade:

$$
\frac{\partial u}{\partial x}+\frac{\partial v}{\partial y}=0
$$

- Conservação de movimento:

$$
\begin{aligned}
& \frac{\partial u}{\partial t}+\frac{\partial u^{2}}{\partial x}+\frac{\partial(u v)}{\partial y}=-\frac{\partial p}{\partial x}+\frac{1}{R e}\left(\frac{\partial^{2} u}{\partial x^{2}}+\frac{\partial^{2} u}{\partial y^{2}}\right)+\frac{g_{x}}{F r^{2}} \\
& \frac{\partial v}{\partial t}+\frac{\partial(u v)}{\partial x}+\frac{\partial v^{2}}{\partial y}=-\frac{\partial p}{\partial y}+\frac{1}{R e}\left(\frac{\partial^{2} v}{\partial x^{2}}+\frac{\partial^{2} v}{\partial y^{2}}\right)+\frac{g_{y}}{F r^{2}}
\end{aligned}
$$

\subsection{Escoamentos Viscosos}

Uma possível classificação dos escoamento, visando a simplificação das equações de conservação, pode ser feita levando-se em conta a importância dos efeitos viscosos, ou seja, quando estes efeitos predominam e se estendem por todo o campo, têm-se os escoamentos com baixo número de Reynolds.

Como foi visto, o número de Reynolds pode ser expresso como

$$
R e=\frac{\text { forças inerciais }}{\text { forças viscosas }}=\frac{\rho_{0} L U}{\mu_{0}}=\frac{L U}{\nu_{0}} .
$$

Quando as forças viscosas são predominantes, isto é, $R e<<1$, o escoamento é denominado viscoso.

\subsection{Condições Iniciais e de Contorno}

A escolha das condições iniciais e de contorno é fundamental para a formulação dos problemas modelados por equações diferenciais parciais, pois o comportamento físico da solução e a solução numérica dependem da escolha apropriada destas condições. Para as condições iniciais deve-se conhecer, em $t=0$, a distribuição espacial das variáveis dependentes, e para as condições de contorno é preciso ter alguma informação física, para todo $t$, das variáveis dependentes nas fronteiras da região limitando o escoamento. 
Ou seja, as equações (3.15) e (3.16) aplicadas à um campo de velocidade inicial apropriado em um domínio e uma condição sobre a fronteira do domínio, constituem um Problema de Valor Inicial e de Fronteira (PVIF) bem posto (Courant e Hilbert, 1962).

A condição inicial apropriada para as equações (3.5) e (3.8) é a especificação no domínio de um campo inicial de velocidade $\mathbf{u}_{0}$ satisfazendo a equação da continuidade $\nabla \cdot \mathbf{u}_{0}=0$. É muito importante que a equação da continuidade seja satisfeita, pois do contrário o problema contínuo pode não possuir solução clássica (Heywwod e Rannacher, 1986).

Para escoamentos viscosos, incompressíveis e envolvendo superfícies livres existem basicamente quatro tipos de condições de contorno. Nessas condições é preciso ter alguma informação física das variáveis dependentes para todo o tempo $t$ na fronteira da região do escoamento. Pode-se definir os quatro tipos como:

- Condição de aderência completa ou sem escorregamento ("no-slip"): Esta condição significa que o fluido deve ser "colado" à superfície, a qual é conhecida como não-escorregadia ("no-slip"). Quando tem-se uma superfície sólida e sem deslizamento de fluido em um escoamento viscoso, é apropriado definir a velocidade tangencial $u_{\tau}$ do fluido em relação à parede como sendo igual a zero. Desta forma, o fluido que está adjacente à superfície da parede estará em repouso em relação à parede. A componente normal $u_{n}$ da velocidade do fluido também é nula, pois o fluido não pode penetrar na parede.

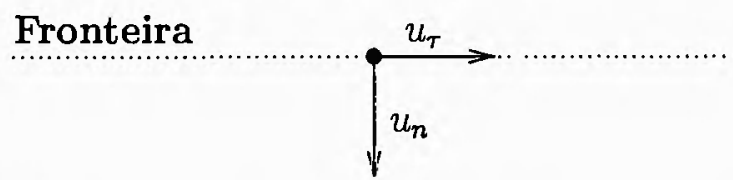

Figura 3.4: Componentes das velocidades em uma fronteira.

- Condição de simetria ou sem aderência ("free-slip"): É usada quando há fronteiras de simetria, ou quando os efeitos da condição de aderência completa não são desejáveis. Neste caso impõe-se $u_{n}=0$ para a componente normal da velocidade, e $\frac{\partial u_{\tau}}{\partial n}=0$ para as componentes tangenciais, onde $n$ é a direção normal à fronteira. Essa condição permite que o fluido deslize livremente sobre a superfície.

- Condição de contorno na superfície livre: Representa uma interface entre o fluido e uma atmosfera. Quando trabalha-se na superfície livre do fluido, é 
necessário impor condições sobre a velocidade e pressão (Batchelor, 1970). Tais condições, considerando que o coeficiente de tensão superficial é nulo (Griebel et al., 1998), se resumem nas equações

$$
\begin{aligned}
& (\mathrm{T} \cdot \mathbf{n}) \cdot \mathbf{n}=0 \\
& (\mathrm{~T} \cdot \mathbf{n}) \cdot \mathrm{m}=0
\end{aligned}
$$

onde para o caso bidimensional, $\mathbf{n}=\left(n_{x}, n_{y}\right)$ é o vetor unitário normal, externo à superfície, $\mathbf{m}=\left(m_{x}, m_{y}\right)$ é o vetor unitário tangente à superfície livre.

Substituindo o tensor de tensões total

$$
\mathrm{T}=-p \mathrm{I}+\tau
$$

nas equações (3.21) e (3.22) obtêm-se as equações em coordenadas cartesianas

$$
\begin{gathered}
-p+\frac{2}{R e}\left[\frac{\partial u}{\partial x} n_{x}^{2}+\frac{\partial v}{\partial y} n_{y}^{2}+\left(\frac{\partial u}{\partial y}+\frac{\partial v}{\partial x}\right) n_{x} n_{y}\right]=0 \\
2 \frac{\partial u}{\partial x} n_{x} m_{x}+2 \frac{\partial v}{\partial y} n_{y} m_{y}+\left[\frac{\partial u}{\partial y}+\frac{\partial v}{\partial x}\right]\left(n_{y} m_{x}+n_{x} m_{y}\right)=0 .
\end{gathered}
$$

Detalhes sobre a aplicação dessas equações nas condições de contorno serão apresentadas mais adiante.

- Condições na entrada e na saída de fluido do domínio: É necessário o conhecimento do escoamento nos contornos da entrada e saída do domínio, ou seja,

- Entrada de fluido ("Inflow"): As condições de contorno na entrada de fluido são dadas por

$$
u_{n}=u_{\text {inflow }} \quad \text { e } \quad u_{\tau}=0
$$

onde $u_{n}$ é a velocidade normal ao contorno e $u_{\tau}$ é a velocidade tangencial ao contorno.

- Saída de fluido ("Outflow"): As condições de contorno na saída de fluido são dadas por

$$
u_{n}=u_{\text {outflow }} \quad \text { e } \frac{\partial n_{-}}{\partial n}=0 .
$$




\section{Método de Projeção e Formulações Implícitas para Equações de Navier-Stokes}

\subsection{Introdução}

O desenvolvimento da mecânica dos fluidos pode ser dividido em três fases:

- Até o fim do século XVII: os trabalhos até esta fase são essencialmente de natureza experimental;

- A partir do século XVIII: foram desenvolvidos trabalhos analíticos por cientistas como Bernoulli, Euler, D'Alembert, Laplace entre outros. Estes trabalhos não descreviam no entanto muitos dos fenômenos observados experimentalmente. No século XIX foram desenvolvidos os primeiros trabalhos sobre Limite de Estabilidade dos Escoamentos (Reynolds);

- No início do século XX: ocorreu notável avanço no desenvolvimento da mecânica dos fluidos com a descoberta e a formulação das teorias de Camada Limite (Prandtll), Sustentação Aerodinâmica (Lancherster-Prandtl) e outros resultados. 
Capítulo 4 - Método de Projeção e Formulações Implícitas para Equações de 38 Navier-Stokes

A resolução das equações da mecânica dos fluidos quando viável exige freqüentemente enorme esforço analítico. Em muitos casos, como já comentado, não se consegue uma solução analítica sendo necessário recorrer à aproximações numéricas obtendo-se assim uma solução numérica.

A solução numérica das equações (3.15) e (3.16), as Equações de Navier-Stokes e da continuidade apresenta várias dificuldades computacionais, motivando assim, diferentes técnicas para se resolvê-las. Uma técnica que despertou e desperta o interesse de muitos pesquisadores é o método de projeção ou passo fracionário, que inicialmente foi proposto com o objetivo de reduzir dificuldades computacionais.

Essa técnica popular para resolver as equações de Navier-Stokes incompressíveis, geralmente simplifica os cálculos computacionais, resumindo os passo para se obter a solução numérica, em um algoritmo prático e eficaz. Diversos pesquisadores têm se dedicado ao estudo dos métodos de projeção (Chorin, 1968), (Kan, 1986), (Gresho, 1990), à análise de sua precisão (Strikwerda e Lee, 1999), (Brown et al., 2001), (Chang et al., 2002) e' (Denaro, 2003) e alguns autores debatem sobre as condições de contorno que devem ser aplicadas (Weinan e Liu, 1995) e (Lee et al., 2001).

Outra dificuldade relacionada à solução numérica das equações de Navier-Stokes, é a utilização de métodos computacionais para as simulações de escoamentos com superfícies livres. Esta dificuldade ocorre, pois para este tipo de escoamento, é necessário especificar o movimento de um corpo de fluido em uma dada região no espaço, isto é, a posição da superfície precisa ser determinada como parte da solução do problema. Existem três formulações que podem ser usadas para resolver esse tipo de problema: a formulação Euleriana, a formulação Lagrangeana e a formulação Lagrangeana-Euleriana. Esta última formulação é uma forma mista das duas primeiras.

$\mathrm{Na}$ formulação Euleriana define-se uma região fixa onde o comportamento do fluido será estudado e as grandezas do escoamento são especificadas como funções da posição e do tempo. Nesta formulação trabalha-se com "Postos de Observação" situados nos pontos do domínio.

$\mathrm{Na}$ formulação Lagrangeana define-se uma região material, ou seja, formada por um conjunto de partículas de fluido. Como nessa formulação o sistema de coordenadas se move continuamente com o fluido, a superfície livre é calculada precisamente.

O método de partículas marcadoras, "Marker and Cell" (MAC)(Harlow e Welch, 1965) utiliza a formulação Euleriana e foi originalmente desenvolvido para resolver 
problemas com superfícies livres, empregando uma.malha fixa, em que as partículas marcadoras (markers) definem a localização da superfície livre do fluido que está em movimento. Outro método Euleriano muito utilizado é o método "Volume of Fluid" (VOF)(Hirt e Nichols, 1981). Um exemplo de métodos Lagrangeanos foi apresentado por (Ramaswamy e Kawahara, 1987), onde a superfície livre é determinada por um sistema de coordenadas que se movimenta com as partículas. Um exemplo de método que utiliza a formulação Lagrangeana-Euleriana, foi descrito por (Hirt et al., 1974). No presente trabalho, adota-se a estratégia do método MAC.

As partículas marcadoras ("marker particules"): usadas no método MAC, movemse com o campo de velocidade. As posições, ou coordenadas Lagrangeanas, de cada partícula $\left(x_{P}^{n}, y_{P}^{n}\right)$ são obtidas por integração numérica para alguma posição inicial $\left(x_{p}^{0}, y_{p}^{?}\right)$ no tempo $t=0$, ou seja,

$$
\begin{aligned}
& x_{P}^{n}=x_{P}^{0}+\int_{0}^{t} u_{P} \mathrm{~d} t \\
& y_{P}^{n}=y_{P}^{0}+\int_{0}^{t} v_{P} \mathrm{dt}
\end{aligned}
$$

onde $u_{P}$ e $v_{P}$ são as velocidades. No método MAC, as equações (4.1) são discretizadas como

$$
\begin{aligned}
& x_{P}^{n+1}=x_{P}^{n}+u_{P} \delta t \\
& y_{P}^{n+1}=y_{P}^{n}+v_{P} \delta t .
\end{aligned}
$$

Desta forma, das equações (4.2) pode-se obter a próxima localização da partícula de fluido durante o escoamento, conhecido o campo de velocidade $(u, v)$.

O método MAC foi apresentado originalmente como um método numérico para escoamentos bidimensionais usando uma malha diferenciada ou deslocada ("staggered grid"), na qual as velocidades $u$ e $v$ são armazenadas nas faces das células, e a pressão $p$ ou outras propriedades do fluido no centro da célula (ver figura 4.1).

$\mathrm{O}$ método $\mathrm{MAC}$ originou vários outros métodos que resolvem problemas com superfícies livres, formando uma importante classe de métodos em DFC, denominados como "métodos para escoamento com superfície livre". Entre esses métodos pode-se destacar o método apresentado por Amsden e Harlow (Amsden e Harlow, 1970), que introduziram o método "Simplified Marker and Cell" (SMAC) para eliminar as dificuldades associadas 
com a aplicação das condições de fronteira do MAC, o método "GENeralized Simplified Marker-And-Cell" (GENSMAC), que constitui uma extensão do método SMAC para domínios bidimensionais arbitrários e apresentado por (Tomé e McKee, 1994).

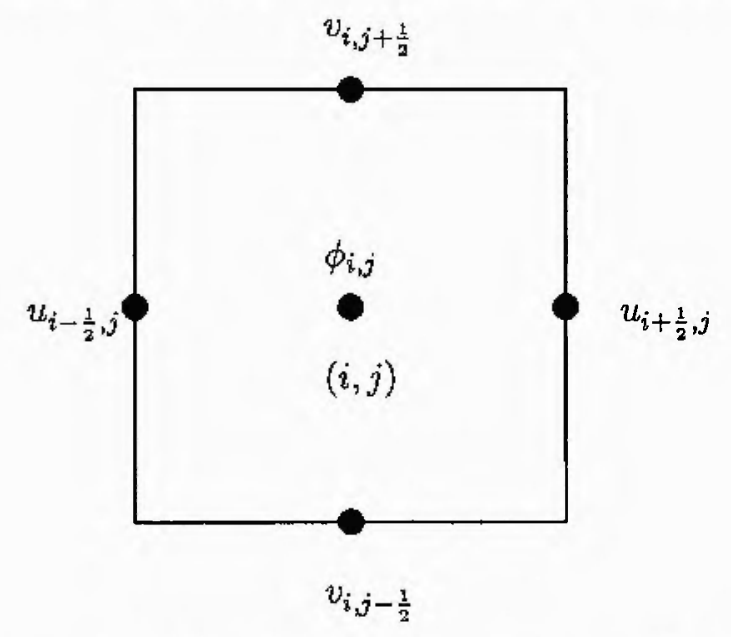

Figura 4.1: Célula utilizada pelo método MAC.

A natureza da discretização temporal do método MAC é explícita utilizando o método de Euler explícito. Alguns autores propuseram modificações na natureza explícita, ou seja, métodos que utilizam técnicas implícitas para resolver as equações de Navier-Stokes. Exemplos dessas modificações são o método "Marker and Cell for Reynolds Low" (MACRL), descrito por (Pracht, 1971) para tratar problemas com número de Reynolds baixo e o método "Semi-Implicit Marker and Cell" (SIMAC) descrito por (Armenio, 1997).

Neste capítulo, uma breve introdução aos métodos de projeção e a aplicação de técnicas implícitas para Equações de Navier-Stokes serão apresentadas.

\subsection{Métodos de Projeção}

Muitos métodos que resolvem problemas de escoamentos viscosos, incompressíveis, newtonianos e transientes com superfícies livres, governados pelas equações (3.15) e (3.16), resolvem essas equações utilizando a formulação de variáveis primitivas (Dennis et al., 1979), e de forma acoplada ou desacoplada (ou seja, o campo de velocidade é calculado separadamente do campo de pressão). Os métodos que resolvem simultanea- 
mente as equações de conservação de movimento e da continuidade em cada passo do tempo, são chamados de métodos de solução acoplados, e os métodos que resolvem as equações separadamente são chamados de métodos segregados.

Os métodos de solução acoplados, por acoplar as equações de conservação de movimento e continuidade, e receber as fortes influências das não-linearidades dos termos convectivos, apresentam maiores dificuldades na sua implementação e um alto custo computacional.

Desta forma, desacoplar as equações é uma metodologia muito utilizada no contexto de resolução das equações de conservação. Quase todos os métodos segregados que resolvem as equações (3.15) e (3.16) em termos de variáveis primitivas usam um método de projeção.

A família dos métodos de projeção fundamentam-se no Teorema da Decomposição de Helmholtz-Hodge.

\subsubsection{Teorema da Decomposição de Helmholtz-Hodge}

O teorema da decomposição de Helmholtz-Hodge (DHH) (Weil, 1940), (Hodge, 1952) também conhecido como teorema de Ladyzhenskaja (Ladyzhenskaja, 1963) é uma ferramenta básica na teoria de soluções generalizadas e também na aproximação numérica de modelos físicos, como as equações Navier-Stokes. Desta forma, quando resolvem-se as equações de Navier-Stokes de forma desacoplada o teorema DHH é utilizado pelo método da projeção.

Teorema 2 (Teorema da Decomposição de Helmholtz-Hodge (DHH)) : Um campo vetorial $\boldsymbol{w}$ definido num dominio $\Omega$ com fronteira suave $\partial \Omega$ é decomposto em um campo gradiente e em um campo solenoidal e paralelo ao contorno. Isto é, o campo $w$ pode ser escrito como:

$$
w=w^{D}+\nabla \psi
$$

onde

$$
w^{D}=\nabla \times b
$$

e b um cumpo vetorial solenoidal.

Definição 1 Dado um campo velorial $\boldsymbol{w}$, se $\nabla \cdot \boldsymbol{w}=0$ diz-se que $\boldsymbol{w}$ é solenoidal. 
Capítulo 4 - Método de Projeção e Formulações Implícitas para Equações de

O campo vetorial $w$ pertence ao espaço de funções e é unicamente determinado quando o divergente (d) e o rotacional (r) são determinados junto com a componente normal (ou tangencial) na fronteira. Desta forma as equações

$$
\begin{aligned}
\nabla \cdot \mathrm{w} & =\mathrm{d}, \quad \\
\nabla \times \mathrm{w} & =\mathrm{r}, \quad \boldsymbol{\mathrm { x }} \in \Omega
\end{aligned}
$$

devem ser satisfeitas com a condição de contorno

$$
\mathbf{n} \cdot \mathbf{w}=w_{n}
$$

ou

$$
\mathbf{n} \times \mathbf{w}=\mathbf{w}_{\tau}, \quad \mathbf{x} \in \partial \Omega
$$

onde $\mathbf{n}$ é o vetor unitário externo a $\partial \Omega$, e $w_{n}$ e $\mathbf{w}_{\tau}$ são as componentes normal e tangencial de $\mathbf{w}$ em $\partial \Omega$, respectivamente.

Assim, conforme (4.5) as componentes da decomposição de w devem satisfazer

$$
\begin{aligned}
\nabla \cdot \nabla \psi & =\mathbf{d} \\
\nabla \times \mathbf{w}^{D} & =\mathbf{r} .
\end{aligned}
$$

A prova da ortogonalidade, existência e unicidade da decomposição (4.3) pode ser encontrada em (Minion, 1996) e (Denaro, 2003).

\subsubsection{Aplicação do Teorema da $\mathrm{DHH}$ para Métodos de Projeção}

De acordo com o teorema da $\mathrm{DHH}$, o campo vetorial $\mathrm{w}^{D}$ representa a componente rotacional, ou seja

$$
\nabla \times \mathbf{w}=\nabla \times \mathbf{w}^{D}
$$

e $\nabla \psi$ representa a parte irrotacional, isto é

$$
0=\nabla \times \dot{\nabla} \psi
$$


A teoria dos métodos de projeção baseiam-se na interpretação das equações de Navier-Stokes como projeções, ou seja, assim como no teorema, w é unicamente determinado e sendo assim, pode-se definir a projeção $M$ por

$$
\mathrm{M}(\mathbf{w})=\mathbf{w}^{D}=\nabla \times \mathbf{b}
$$

e a projeção $\mathrm{N}$ como

$$
\mathrm{N}(\mathbf{w})=\nabla \psi
$$

e portanto

$$
\mathbf{w}=M(w)+N(w)
$$

Note que

$$
\begin{aligned}
\nabla \cdot \mathrm{M}(\mathbf{w}) & =0 \\
\nabla \times \mathrm{N}(\mathbf{w}) & =0
\end{aligned}
$$

e

$$
(I-\mathrm{M})(\mathbf{w})=\mathrm{N}(\mathbf{w})=\nabla \psi
$$

Aplicando o operador divergente em (4.11) e da primeira equação em (4.12) tem-se

$$
\nabla \cdot \mathbf{w}=\nabla \cdot \mathrm{N}(\mathbf{w})=\nabla^{2} \psi
$$

Desta forma, pode-se determinar

$$
\psi=\left(\nabla^{2}\right)^{-1} \nabla \cdot \mathbf{w}
$$

e $\operatorname{assim} N(\mathbf{w})=\nabla\left(\nabla^{2}\right)^{-1} \nabla . w$. Portanto uma forma de expressar o operador $N$ é

$$
N=\nabla\left(\nabla^{2}\right)^{-1} \nabla
$$

Aplicando o operador rotacional em (4.11) e da segunda equação em (4.12) tem-se

$$
\nabla \times \mathbf{w}=\nabla \times M(\mathbf{w})
$$


Capítulo 4 - Método de Projeção e Formulações Implícitas para Equações de De (4.9) e após um pouco de álgebra determina-se

$$
\mathbf{b}=\left(-\nabla^{2}\right)^{-1} \nabla \times \mathbf{w}
$$

e assim, $M(\mathbf{w})=\nabla \times\left(-\nabla^{2}\right)^{-1} \nabla \times \mathbf{w}$. Finalmente, o operador $M$ pode ser escrito como

$$
\mathrm{M}=\nabla \times\left(-\nabla^{2}\right)^{-1} \nabla \times
$$

Segundo (Chorin, 1968), utilizando os operadores M e N, a pressão e a velocidade podem ser desacopladas das equações de Navier-Stokes. Portanto os métodos de projeção resolvem as equações de Navier-Stokes de forma desacoplada. Devido às dificuldades de se aplicar os operadores $\mathrm{M}$ e $\mathrm{N}$ diretamente, utiliza-se o teorema (2). Resumidamente, essa aplicação pode ser descrita da seguinte forma.

A aceleração Euleriana a (Denaro, 2003) baseada na equação (3.15), desconsiderando à ação gravitacional, é definida como

$$
\mathbf{a}:=\frac{\partial \mathbf{u}}{\partial t}=-\nabla \cdot(\mathbf{u u})+\frac{1}{l e} \nabla^{2} \mathbf{u}-\nabla p
$$

onde a é um campo solenoidal devido à condição de incompressibilidade dada pela equação (3.16). Considere agora o campo vetorial ã determinado pelos termos convectivos e difusivos de $\mathbf{u}$, isto é:

$$
\tilde{\mathbf{a}}:=-\nabla \cdot(\mathbf{u u})+\frac{1}{R e} \nabla^{2} \mathbf{u} .
$$

Portanto, a equação (4.20) pode ser reescrita como

$$
\tilde{\mathbf{a}}=\mathbf{a}+\nabla p
$$

Desta maneira, a equação (4.22) tem a forma da decomposição (4.3), onde, $\tilde{\mathbf{a}}=\mathbf{w}$, $\mathrm{a}=\mathrm{w}^{D}$ e $\nabla p=\nabla \psi$. Porém, para que a equação (4.22) satisfaça o teorema DHH, os campos vetoriais devem satisfazer as equações (4.6), reescritas como

$$
\begin{aligned}
& \nabla \cdot \nabla p=\nabla \cdot \tilde{\mathbf{a}} \\
& \nabla \times \mathbf{a}=\nabla \times \tilde{\mathbf{a}}
\end{aligned}
$$


com

$$
\mathbf{n}, \tilde{\mathbf{a}}=\tilde{a_{n}} \quad \text { ou } \quad \mathbf{n} \times \tilde{\mathbf{a}}=\tilde{\mathbf{a}}_{\tau}, \quad \mathbf{x} \in \partial \Omega .
$$

Basicamente, a idéia principal do método de projeção é utilizar a equação do movimento (3.15) para determinar um campo de velocidade provisório (ou tentativo), ou seja, define-se a equação

$$
\frac{\partial \tilde{\mathbf{u}}}{\partial t}=-\nabla \cdot(\mathbf{u u})+\frac{1}{R e} \nabla^{2} \mathbf{u}-\nabla \bar{p}+\frac{1}{F r^{2}} \mathbf{g},
$$

onde $\bar{p}$ é uma aproximação para a pressão (ou uma pressão provisória). Em muitos casos, a velocidade ũ não é solenoidal, isto é, não satisfaz a equação da continuidade (3.16), pois em geral $p \neq \tilde{p}$.

Observando as equações (3.15) e (3.16), para o caso bidimensional, nota-se que existem três equações e três incógnitas $(u, v$ e $p)$, onde a pressão só aparece na equação (3.15). A função da pressão em escoamentos incompressíveis é fazer com que o campo de velocidade satisfaça a equação da continuidade (3.16). Portanto é necessário calcular a pressão no próximo passo. Para isto uma equação elíptica é resolvida para que a equação (3.16) seja satisfeita e a pressão determinada (Gresho e Sani, 1987). A importância dessa equação elíptica para a pressão, é que ela faz a ligação entre as equações da conservação de movimento e da continuidade. Alguns métodos resolvem diretamente essa equação elíptica para a pressão, como (Henshaw, 1994), outros utilizam um potencial auxiliar relacionado à pressão.

Após o cálculo do potencial auxiliar, o campo de velocidade final e a pressão são calculados. Basicamente, os métodos de projeção seguem um esquema, que pode ser resumido como:

- Passo da velocidade intermediária: Resolver a equação (4.24) para o campo de velocidade intermediário ũ. Em algumas variações do método de projeção, o gradiente de pressão provisório da equação (4.24) é eliminado (Kim e Moin, 1985), em outros, o gradiente é conservado (Bell et al., 1989), (Gresho, 1990), (Bell et al., 1991), (Perot, 1993), (Botella, 1997) e (Hugues e Randriamampianina, 1998). Os métodos de projeção que desprezam o gradiente de pressão são conhecidos como métodos de projeção sem-pressão ("pressure-free projection methods"), e os métodos que o conservam, métodos de projeção com acréscimo da pressão ("incremental-pressure projection methods"). 
Capítulo 4 - Método de Projeção e Formulações Implícitas para Equações de

- Passo da projeção: Resolver uma equação elíptica para a pressão ou para um potencial auxiliar $\psi$ (ou campo projetor $\psi$ ). Vários autores, como (Petersson, 2001), (Johnston e Liu, 2002) e outros, têm se preocupado com as condições de contorno adequadas para resolver essa equação elíptica.

- Passo de atualização: Calcular o campo de velocidade e pressão final utilizando o campo projetor $\psi$.

As condições de contorno apropriadas para a solução das equações do algoritmo acima serão dadas de acordo com os métodos utilizados (Gresho e Chan, 1990).

No próximo capítulo, a formulação matemática do método da projeção será aplicada para o método estudado no presente trabalho, utilizando-se um método de projeção sempressão e um método de projeção com acréscimo da pressão.

\subsubsection{Aplicação do Teorema DHH na Forma Discreta para o Método MAC}

Dado um domínio computacional $\Omega$, seja $(i, j)_{\Omega}$ um ponto na $i$-ésima coluna e $j$ ésima linha contido em $\Omega$. A fronteira $\partial \Omega$ consiste em um conjunto de arestas $\xi_{k}$. Define-se também, $D(\mathbf{u})_{i, j}$ como o operador divergente e $\widehat{G}\left(\hat{\psi}_{i, j}\right.$ o operador gradiente, ambos na forma discreta. O teorema $\mathrm{DHH}$ pode ser estendido para a forma discreta, $\mathrm{e}$ nesta forma tem a seguinte redação:

\section{Teorema 3 (Teorema da DHH na forma discreta)}

Seja $\Omega$ um dominio computacional contendo células com indices $(i, j)_{\Omega}$ com fronteira dada por um conjunto de aresta $\xi_{k}$. Dado um campo vetorial discreto $u_{i, j}$ e um fluxo $F_{k}(u)$ definido na fronteira, $u_{i, j}$ pode ser unicamente decomposto na forma

$$
u_{i, j}=u_{i, j}^{D}+G(\psi)_{i, j}
$$

onde

$$
\begin{aligned}
& D\left(u^{D}\right)_{i, j}=0 \quad \text { em } \quad \Omega, \\
& F_{k}\left(u^{D}\right)=0 \text { na fronteira } \partial \Omega \text {. }
\end{aligned}
$$


Desde que esta decomposição exista, um operador de projeção na forma discreta pode ser definido como

$$
\mathrm{M}(\mathbf{u})_{i, j}=\mathbf{u}_{i, j}^{D}
$$

Logo, utilizando o teorema, tem-se

$$
\mathrm{M}(\mathbf{u})_{i, j}=\mathbf{u}_{i, j}^{D}=\mathbf{u}_{i, j}-G(\psi)_{i, j}
$$

onde $\psi$ é a solução da equação de Poisson discreta

$$
D G(\psi)_{i, j}=D(\mathbf{u})_{i, j}
$$

De acordo com o método MAC, utiliza-se um campo de velocidade intermediário $\tilde{\mathbf{u}}$, e portanto pode-se aplicar o teorema (3) e as projeções discretas M e N para o vetor ũ. Para se provar o teorema (3), deve-se também utilizar o Teorema da Divergência Discreta (Minion, 1996). Mais detalhes podem ser encontrados em (Minion, 1994).

\subsection{Formulações Implícitas para Equações de Navier- Stokes}

Métodos baseados nos métodos de projeções e no método MAC podem resolver as equações de Navier-Stokes utilizando formulações explícitas ou implícitas. Como mencionado anteriormente, a principal motivação para o uso de métodos implícitos para as equações de Navier-Stokes é explorar a propriedade de estabilidade associada a esses métodos. Em muitos problemas, quando as restrições de estabilidade são relaxadas, o tamanho do passo temporal permitido torna-se substancialmente maior. Isto é muito importante do ponto de vista prático, pois um valor de passo temporal extremamente restritivo faz com que o tempo de simulação seja longo. Detalhes sobre as propriedades de estabilidade dos métodos implícitos foram descritos na seção 2.4 .

Geralmente, três técnicas são utilizadas para aliviar as restrições de estabilidade impostas pelas formulações explícitas. A seguir, serão apresentadas essas técnicas e alguns exemplos de métodos que utilizam formulações implícitas para resolver as equações de conservação. 
Capítulo 4 - Método de Projeção e Formulações Implícitas para Equações de

1. Discretizar os termos viscosos (ou difusivos) no nivel de tempo $n+1$ Nessa técnica, os termos viscosos das equações de Navier-Stokes para o cálculo do campo de velocidade intermediário, escritos em coordenadas cartesiana bidimensionais como

$$
\left(\frac{\partial^{2} u}{\partial x^{2}}+\frac{\partial^{2} u}{\partial y^{2}}\right)
$$

$$
\left(\frac{\partial^{2} v}{\partial x^{2}}+\frac{\partial^{2} v}{\partial y^{2}}\right)
$$

são tratados implicitamente por algum método, como por exemplo implícito regressivo, Crank-Nicolson ou ADI.

O método SIMAC desenvolvido por (Armenio, 1997) é um método baseado no método MAC que utiliza essa estratégia de discretizar no nível de tempo $n+1$ apenas os termos viscosos. Seguindo os passos do esquema do método da projeção, SIMAC resolve o seguinte conjunto de equações:

- No Passo 1, a equação

$$
\frac{\partial \tilde{\mathbf{u}}}{\partial t}-\frac{1}{2 R e} \nabla^{2} \tilde{\mathbf{u}}=\left\{-\frac{3}{2} \nabla \cdot(\mathbf{u u})^{n}+\frac{1}{\bar{L}} \vee \cdot(\mathbf{u u})^{\cdots-i}+\frac{1}{2 R e} \nabla^{2} \mathbf{u}^{n}-\nabla \psi^{n}+\frac{1}{F r^{2}} \mathbf{g}^{n}\right\},
$$

é utilizada para o cálculo do campo de velocidade intermediária.

- No Passo 2 , obtêm-se a seguinte equação para o cálculo do campo projetor $\psi$

$$
\nabla^{2} \psi^{n+1}=\frac{1}{\delta t} \nabla \cdot \tilde{\mathbf{u}}+\nabla^{2} \psi^{n}
$$

com as apropriadas condições de contorno (Armenio, 1997).

- No Passo 3, atualizam-se os campos de velocidade e de pressão após o cálculo do campo projetor $\psi$, pelas equações

$$
\frac{\mathbf{u}^{n+1}-\tilde{\mathbf{u}}}{\delta t}=-\left(\nabla, \eta^{n+1}-\nabla \psi^{n}\right),
$$

$\mathrm{e}$

$$
p^{n+1}=\psi^{n+1}+\frac{\delta t}{2 R e}\left(\nabla^{2} \psi^{n+1}-\nabla^{2} \psi^{n}\right)
$$

Vários autores como (Deville, 1975), (Manna e Vacca, 1999), (Street, 2003), e outros, também utilizam a técnica de discretizar no nível de tempo $n+1$ apenas os termos viscosos. 
2. Discretizar os termos viscosos e os convectivos no nível de tempo $n+1$ Outra técnica bastante utilizada, é, além de discretizar os termos viscosos, também discretizar os termos convectivos da equação para o cálculo do campo de velocidade intermediário no nível de tempo $n+1$. Em muitos casos é necessário aplicar uma linearização para poder utilizar essa técnica. Os termos convectivos podem ser escritos em coordenadas cartesianas como

$$
\frac{\partial u^{2}}{\partial x}+\frac{\partial(u v)}{\partial y}
$$

e

$$
\frac{\partial(u v)}{\partial x}+\frac{\partial v^{2}}{\partial y}
$$

A discretização implícita dos termos convectivos pode causar algumas dificuldades devido às não-linearidades. Soluções para esse problema são descritos por diversos autores como (Kan, 1986) e (Kim et al., 2002), que utilizaram um método de solução segregado nessa técnica de discretização implícita dos termos convectivos e viscosos.

\section{Discretizar todas as derivadas espaciais no nível de tempo $n+1$}

Essa técnica é mais utilizada para métodos de solução acoplada, pois todas as derivadas espaciais da equação (3.15) são tratadas implicitamente e adota-se um método iterativo de solução. O método "Symetric-Coupled Gauss-Seidel" (SCGS) apresentado por (Vanka, 1986) é um exemplo da aplicação desse técnica. Outros exemplos, foram descritos por (Caretto et al., 1972), (Turek, 1996), (Bansch, 1998), (Vuik et al., 2000), (Langtangen e Winther, 2002), (Bijl et al., 2002) e outros.

O método MACRL proposto por (Pracht, 1971) é uma variação desta técnica implicita, pois os termos viscosos e o gradiente de pressão são resolvidos implicitamente, e como este método foi proposto para escoamentos viscosos, os termos convectivos não são tratados implicitamente. A aplicação deste método resulta em um sistema acoplado das equações de conservação de movimento e de Poisson para a pressão, e a cada ciclo computacional, aplica-se um método iterativo, tipo Gauss-Seidel, para resolver o sistema resultante. Detalhes em (Pracht, 1971).

Métodos como "Semi-IMPlicit Linked Equations" (SIMPLE) (Patankar e Spalding, 1972), "Semi-IMPlicit Linked Equations Revised" (SIMPLER) (Patankar, 1980), "Pres- 
Capítulo 4 - Método de Projeção e Formulações Implícitas para Equações de 50 Navier-Stokes sure Implicit Momentum Explicit" (PRIME) (Maliska, 1981) entre outros são exemplos de métodos que utilizam outras técnicas para aplicar as formulações implícitas. Um resumo sobre a metodologia desses métodos pode ser encontrada em (Maliska, 1995).

Portanto, a aplicação de umas dessas técnicas, tem como objetivo, relaxar as restrições de estabilidade impostas por métodos explícitos. Como foi visto na seção 2.4, métodos implícitos são incondicionalmente estáveis, motivando sua utilização em discretizações implícitas dos termos viscosos. Desta forma, o tamanho do passo temporal $\delta t$ permitido para o método ser estável, será maior que o permitido pelos métodos explícitos.

Entretanto, a utilização de métodos implícitos, pode ocasionar problemas em algumas situações. Além das dificuldades de implementação, pois a utilização desses métodos exigem cálculos mais extensos, outro problema pode ser em relação à precisão dos resultados, ou seja, a precisão do resultado numérico é reduzida conforme se aumenta o valor de $\delta t$. Desta forma, um cuidado que deve-se ter na utilização de métodos implícitos, é o de efetivamente limitar o tamanho do passo temporal, não permitindo que este seja tão grande a ponto de prejudicar a precisão numérica do método. Portanto, a aplicação de métodos implícitos, elimina a restrição de estabilidade do método explícito mas deve-se fazer testes com diversos valores de $\delta t$, para certificar-se que a solução numérica não perde a qualidade. Essa discussão será retomada neste trabalho no capítulo de resultados numéricos.

Comparações entre as técnicas apresentadas anteriormente podem ser vistas em (Turek, 1996) e (Briley e McDonald, 2001). 


\section{Formulações Implícitas na Metodologia GENSMAC}

\subsection{Introdução}

O método "GENeralized Simplified Marker-And-Cell" (GENSMAC) desenvolvido por (Tomé e McKee, 1994) é uma extensão do método SMAC para domínios bidimensionais arbitrários. Logo após, (Castelo et al., 1999) incorporaram tensão superficial no código GENSMAC e em seguida (Tomé et al., 2000) propuseram uma extensão do código GENSMAC para resolver escoamentos em geometrias com simetria radial. Outras extensões do método GENSMAC foram descritas por (Ferreira, 2001) para escoamentos em regime turbulentos, (Sabatini, 2002) para escoamentos não-isotérmicos, (Souza, 2002) para problemas de escoamentos multifásicos e por (Grossi, 2003), (Silva, 2003) e (Doricio, 2003) para problemas de escoamentos não-newtonianos e viscoelásticos.

O método GENSMAC utiliza um tratamento explícito, e para problemas onde as forças inerciais são predominantes sobre as forças viscosas (quando $R e>1$ ) este método produz bons resultados com uma restrição, no passo temporal, não muito severa. No entanto, quando as forças viscosas são predominantes (quando $R e<1$ ), devido às restrições relacionadas com a estabilidade, os métodos explícitos tornam-se ineficientes. 
Desta forma, uma solução é o tratamento implícito dos termos viscosos, isto é, utilizar a técnica descrita na seção 4.3, e assim constroem-se métodos semi-implícitos para resolver as equações de Navier-Stokes. Neste trabalho, os termos viscosos foram inicialmente tratados implicitamente, pelos métodos implícito regressivo (ou Euler implícito), Crank-Nicolson ou um preditor-corretor. Os métodos de projeção descritos em (4.2) foram aplicados para o tratamento implícito dos termos viscosos.

Como o objetivo principal deste trabalho é propor uma modificação na metodologia GENSMAC, neste capítulo será apresentado a formulação matemática desta metodologia, e logo após, as modificações utilizando as formulações implícitas.

\subsection{Método GENSMAC}

O método GENSMAC utiliza uma variante do método de projeção de Chorin (Chorin, 1968) para sua formulação matemática. O procedimento de solução do método GENSMAC será apresentado utilizando a teoria dos métodos de projeção. Para este desenvolvimento, utiliza-se uma forma discreta para o método de projeção (Kim e Moin, 1985).

Inicialmente, considera-se uma pressão tentativa $\vec{p}$, imposta como sendo zero na região de fluido, e calculada para as superfícies livres através das condições de contorno apropriadas. Logo, a partir da equação (3.15) define-se um campo de velocidade intermediário num tempo $t=t_{0}$, como

$$
\left.\frac{\partial \tilde{\mathbf{u}}}{\partial t}\right|_{t=t_{0}}=\left.\left\{-\nabla \cdot(\mathbf{u u})-\nabla \tilde{p}+\frac{1}{R e} \nabla^{2} \mathbf{u}+\frac{1}{F r^{2}} \mathbf{g}\right\}\right|_{t=t_{0}} .
$$

Geralmente, o campo de velocidade $\tilde{\mathbf{u}}$ não é solenoidal, pois $\hat{p} \neq p$. Para $t=t_{0}$, considera-se $\mathbf{u}\left(\mathbf{x}, t_{0}\right)=\tilde{\mathbf{u}}\left(\mathbf{x}, t_{0}\right)$ satisfazendo as condições de contorno.

Utilizando o teorema DHH, descrito na subseção 4.2.1, após subtrair a equação (5.1) de (3.15), repetida aqui

$$
\frac{\partial \mathbf{u}}{\partial t}+\nabla \cdot(\mathbf{u u})=-\nabla p+\frac{1}{R e} \nabla^{2} \mathbf{u}+\frac{1}{F r^{2}} \mathbf{g}
$$


para o tempo $t-t_{0}$, tem-se

$$
\left.\frac{\partial(\mathbf{u}-\tilde{\mathbf{u}})}{\partial t}\right|_{t=t_{0}}=-\left.\nabla P\right|_{t=t_{0}},
$$

onde $P=p-\vec{p}$. Aplicando o rotacional em ambos os lados de (5.2)

$$
\left.\frac{\partial}{\partial t}(\nabla \times(\mathbf{u}-\tilde{\mathbf{u}}))\right|_{t=t_{0}}=0
$$

pois da análise vetorial, tem-se $\nabla \times \nabla P=0$. Utilizando a idéia de um método de projeção discreto, a derivada (5.3) pode ser aproximada por diferenças progressivas, tal que

$$
\frac{\nabla \times\left.(\mathbf{u}-\tilde{\mathbf{u}})\right|_{t_{0}+\delta t}-\nabla \times\left.(\mathbf{u}-\tilde{\mathbf{u}})\right|_{t_{0}}}{\delta t} \simeq 0
$$

Como em $t=t_{0}, \mathbf{u}\left(\mathbf{x}, t_{0}\right)=\tilde{\mathbf{u}}\left(\mathbf{x}, t_{0}\right)$, reescreve-se (5.4) como

$$
\nabla \times\left.(\mathbf{u}-\tilde{\mathbf{u}})\right|_{t_{0}+\delta t} \simeq 0
$$

O significado físico de (5.5) é que as vorticidades associadas à $\mathbf{u}$ e $\tilde{\mathbf{u}}$, no tempo $t=t_{0}+\delta t$, são idênticas. Ainda mais, o escoamento com respeito ao campo $(\mathbf{u}-\tilde{\mathbf{u}})$ é irrotacional. Isso equivale a dizer que existe uma função escalar $\psi$, chamada potencial de velocidades (Ames, 1972), de maneira que no tempo $t_{0}+\delta t$

$$
\mathbf{u}=\tilde{\mathbf{u}}-\nabla \psi
$$

Assumindo que o campo vetorial $\mathbf{u}(\mathbf{x}, t)$ é solenoidal, quando aplica-se o divergente em ambos os lados da equação (5.6), tem-se

$$
\nabla^{2} \psi(\mathbf{x}, t)=\nabla \cdot \tilde{\mathbf{u}}(\mathbf{x}, t)
$$

Note que para resolver (5.7) no tempo atual $t=t_{0}+\delta t$, não se conhece a configuração do fluido no domínio. Desta forma, de acordo com (Ferreira, 2001), o que se faz, então, é resolver a equação de Poisson no tempo $t=t_{0}$, argumentando que o passo $\delta t$ é pequeno e, portanto, as configurações em $t_{0}$ e $t_{0}+\delta t$ diferem muito pouco. Portanto, no método GENSMAC, admite-se que a equação (5.7) é resolvida para $t=t_{0}$. A pressão $p(\mathrm{x}, t)$ é 
atualizada utilizando-se a equação (5.2), pois

$$
\left.\frac{\partial(\mathbf{u}-\tilde{\mathbf{u}})}{\partial t}\right|_{t=t_{0}}=-\left.\nabla(p-\tilde{p})\right|_{t=t_{0}}
$$

Mais uma vez, aproximando a derivada da equação (5.8) por diferenças progressivas,

$$
\frac{\left.(\mathbf{u}-\tilde{\mathbf{u}})\right|_{t_{0}+\delta t}-\left.(\mathbf{u}-\tilde{\mathbf{u}})\right|_{t_{0}}}{\delta t} \simeq-\left.\nabla(p-\tilde{p})\right|_{t=t_{0}} .
$$

Desta forma,

$$
\frac{\mathbf{u}\left(\mathbf{x}, t_{0}+\delta t\right)-\tilde{\mathbf{u}}\left(\mathbf{x}, t_{0}+\delta t\right)}{\delta t}=-\nabla\left(p\left(\mathbf{x}, t_{0}\right)-\bar{p}\left(\mathbf{x}, t_{0}\right)\right) .
$$

Utilizando (5.6), tem-se

$$
-\left.\frac{\nabla \psi}{\delta t}\right|_{t_{0}+\delta t}=-\left.\nabla(p-\dot{p})\right|_{t_{0}} \Rightarrow \nabla\left(\left.\frac{\psi}{\delta t}\right|_{t_{0}+\delta t}-\left.(p-\bar{p})\right|_{t_{0}}\right)=0
$$

$\mathrm{E}$ isto implica que

$$
\left.\frac{\psi}{\delta t}\right|_{t_{0}+\delta t}-\left.(p-\bar{p})\right|_{t_{0}} \simeq F(t)
$$

Assumindo que $F(t)=0$, a equação para atualização da pressão é escrita como

$$
p=\tilde{p}+\frac{\psi}{\delta t}
$$

Portanto, esta é a formulação matemática do método GENSMAC.

\subsubsection{Classificação das Células}

Como o método GENSMAC foi originalmente criado para resolver problemas com superfícies livres uma estratégia de classificação de células da malha é utilizada para representar a movimentação do fluido. Essa classificação, identifica se uma célula contém ou não fluido, se pertence à superfície livre, se faz parte da entrada ou saída do domínio ou está no contorno rígido. A figura 5.1 ilustra a classificação das células na 
malha em um dado instante para um escoamento bidimensional (as células vazias estão em branco). A classificação das células é convencionada como:

- Células Vazias/Empty (E): São células vazias que não contém fluido;

- Células Cheias/Full (F): São células cheias de fluido e que não possuem nenhuma face em contato com as células vazias;

- Células de Superfície/Surface (S): São células que contém fluido mas possuem uma ou mais faces em contato com células vazias;

- Células do Contorno/Boundary (B): São células que pertencem ao domínio rígido;

- Células do Injetor/Inflow (I): São células que pertencem à fronteira de entrada do fluido no domínio;

- Células do Ejetor/Outflow (O): São células que pertencem à fronteira de saída do fluido no domínio.

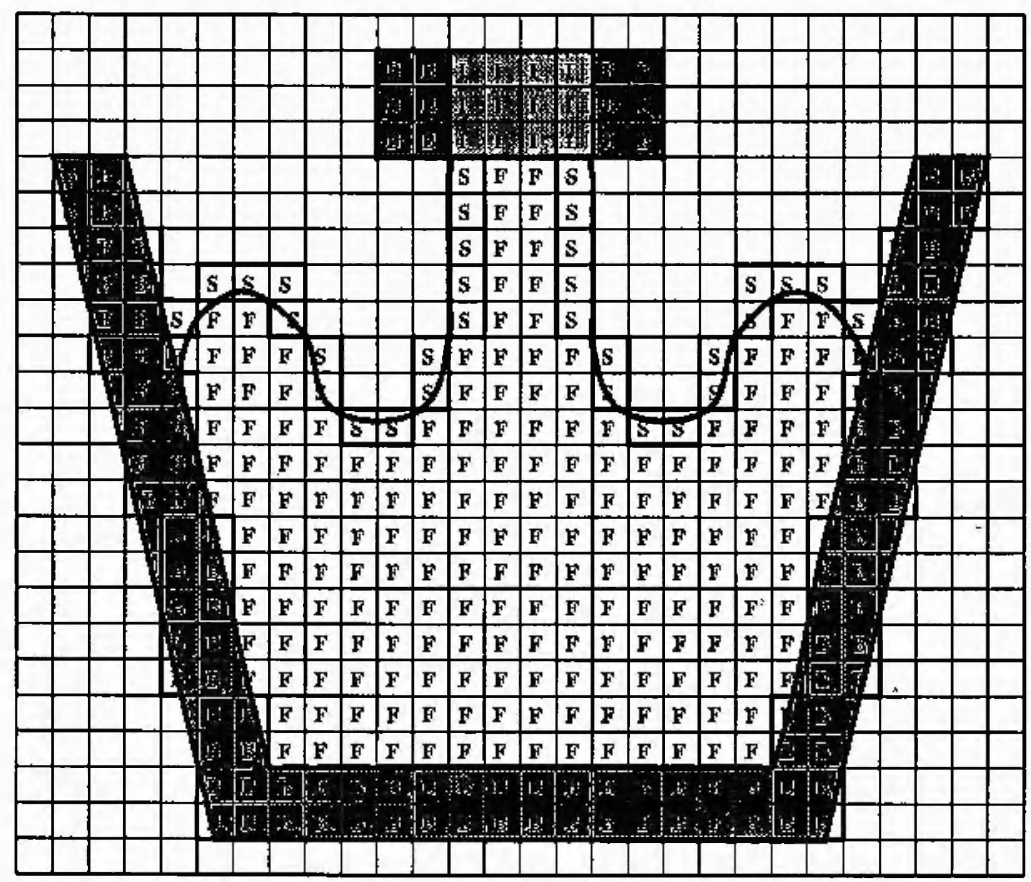

Figura 5.1: Tipos de células.

O tipo de malha utilizada é a mesma do método MAC, isto é, no método GENSMAC, utiliza-se a malha diferenciada ("staggered grid"), que pode ser vista na figura 4.1. 


\subsubsection{Condições Iniciais e de Contorno}

Para as variáveis primitivas velocidade e pressão, as condições iniciais para o método GENSMAC são prescritas no início dos cálculos (Tomé e McKee, 1994). As condições de contorno utilizadas na metodologia GENSMAC são as mesmas apresentadas na seção 3.7. Em particular, as condições de contorno para as superfícies livres requerem uma atenção especial. Dado um domínio $\Omega$, um escoamento nesse domínio pode ser dividido em duas partes: o interno e o externo ao contorno. O interno é preenchido por fluido, e o externo por uma atmosfera inerte. Em algumas variações da metodologia GENSMAC (Souza, 2002), o domínio externo é preenchido por outro fluido. Neste trabalho, o fluido está sempre em contato com uma atmosfera inerte. Portanto, considerando o coeficiente de tensão superficial nulo, as variáveis primitivas velocidade e pressão nas superfícies livres são fornecidas a partir das equações apresentadas na seção 3.7, repetidas aqui

$$
\begin{gathered}
-p+\frac{2}{R e}\left[\frac{\partial u}{\partial x} n_{x}^{2}+\frac{\partial v}{\partial y} n_{y}^{2}+\left(\frac{\partial u}{\partial y}+\frac{\partial v}{\partial x}\right) n_{x} n_{y}\right]=0 \\
2 \frac{\partial u}{\partial x} n_{x} m_{x}+2 \frac{\partial v}{\partial y} n_{y} m_{y}+\left[\frac{\partial u}{\partial y}+\frac{\partial v}{\partial x}\right]\left(n_{y} m_{x}+n_{x} m_{y}\right)=0
\end{gathered}
$$

\subsubsection{Algoritmo do Método GENSMAC}

Utilizando uma formulação discreta no tempo, as equações da formulação matemática do método GENSMAC são reescritas, resumidamente, através do seguinte algoritmo:

- Passo 1: Seja $\tilde{p}\left(\mathbf{x}, t_{n}\right)$ um campo de pressão arbitrário (ou tentativo) que satisfaça as condições de contorno na superfície livre. Esse campo de pressão é calculado de acordo com a equação (5.14) e as velocidades nas superfícies livres por (5.15).

- Passo 2: Conhecido o campo de pressão $\tilde{p}$ calcular um campo de velocidade intermediário $\tilde{\mathbf{u}}(\mathbf{x}, t)$ em $t_{n+1}=t_{n}+\delta t$ satisfazendo a equação:

$$
\frac{\partial \tilde{\mathbf{u}}}{\partial t}=\left\{-\nabla \cdot(\mathbf{u} \mathbf{u})-\nabla \tilde{p}+\frac{1}{R e} \nabla^{2} \mathbf{u}+\frac{1}{F r^{2}} \mathbf{g}\right\}_{t=!_{n}},
$$

definida a partir de (3.15). 
- Passo 3: Resolver a equação de Poisson para o potencial $\psi$.

$$
\nabla^{2} \psi^{n+1}=\nabla \cdot \tilde{\mathbf{u}}
$$

utilizando as seguintes condições de contorno:

- Condições homogéneas do tipo Neumann no contorno rígido, ou seja

$$
\frac{\partial \psi}{\partial n}=0
$$

Esta condição é utilizada também, nas regiões de entrada de fluido, caso existam.

- Condições homogéneas do tipo Dirichlet na superfície livre, ou seja

$$
\psi=0
$$

Esta condição é também utilizada nas regiões de saída de fluido, caso existam.

- Passo 4: Atualizar o campo de velocidade final

$$
\mathbf{u}^{n+1}=\tilde{\mathbf{u}}-\nabla \psi^{n+i}
$$

- Passo 5: Atualizar o campo de pressão final

$$
p^{n+1}=\bar{p}+\frac{\psi^{n+1}}{\delta t}
$$

- Passo 6: Determinar as novas posições das partículas marcadoras virtuais resolvendo as EDOs:

$$
\frac{\mathrm{dx}}{\mathrm{dt}}=u \quad \text { e } \quad \frac{\mathrm{dy}}{\mathrm{dt}}=v
$$

Essas equações são discretizadas pelo método de Euler explícito, ou seja,

$$
\frac{x^{n+1}-x^{n}}{\delta t}=u^{n+1} \quad \text { e } \quad \frac{y^{n+1}-y^{n}}{\delta t}=v^{n+1}
$$

Os passos acima formam um ciclo computacional, a partir de um tempo inicial $t_{n}$, para o cálculo das variáveis primitivas num tempo posterior $t_{n+1}=t_{n}+\delta t$. 


\subsubsection{Condição de Estabilidade do Método GENSMAC}

Assim como no método MAC, o método GENSMAC lida com equações não-lineares, dificultando a análise analítica da estabilidade. Desta forma, pode-se argumentar pelo estudo das equações linearizadas utilizando um método de análise de estabilidade, como os apresentados na seção 2.4. Portanto o critério de estabilidade empregado é uma condição necessária, mas não suficiente.

O uso de integração temporal explícita impõe severas restrições aos valores permitidos de $\delta t$ em problemas onde os termos viscosos (4.30) e (4.31) da equação (3.15) são predominantes. Em particular, esse tipo de problema ocorre quando o escoamento apresenta número de Reynolds baixo, os chamados creep flow.

A restrição imposta pelo tratamento explícito dos termos viscosos é

$$
\delta t_{v i s c} \leq \frac{R e}{2}\left(\frac{1}{(\delta x)^{2}}+\frac{1}{(\delta y)^{2}}\right)^{-1}
$$

Essa restrição é facilmente derivada da equação de difusão de calor (2.9) apresentada na seção 2.2, utilizando o método explícito (2.10). Sabe-se que a condição de estabilidade do método explícito para essa equação é

$$
\sigma_{x}+\sigma_{y} \leq \frac{1}{2}
$$

onde $\sigma_{x}$ e $\sigma_{y}$, apresentados na seção 2.2, são os números de difusão. Para $\alpha=\frac{1}{R e}$ em $\sigma_{x}$ e $\sigma_{y}$, tem-se a restrição (5.22).

Outra restrição de estabilidade do método GENSMAC relaciona o valor do passo temporal com o espaçamento da malha e com uma velocidade de referência. Ou seja, o fluido, ao longo do escoamento, não pode percorrer uma distância maior que o comprimento de uma célula a cada passo no tempo, pois caso isso ocorra, pode-se perder informações sobre as propriedades que estão sendo transportadas. Essa restrição, para o método GENSMAC, exige que

$$
\delta t_{C F L x} \leq \frac{1}{2}\left(\frac{\delta x}{|u|_{\max }}\right)
$$

e

$$
\delta t_{C F L y} \leq \frac{1}{2}\left(\frac{\delta y}{|v|_{\max }}\right)
$$


5.3 Método de Projeção-1 para Formulações Implícitas na metodologia GENSMAC 59 onde $|u|_{\text {max }}$ e $|v|_{\text {max }}$ são os módulos máximos das velocidades nas direções $x$ e $y$, respectivamente.

Essa restrição é derivada da condição Courant-Friedrichs-Lewy (CFL) (Courant et al., 1967), que exige que

$$
\eta_{x} \leq 1 \quad \text { e } \quad \eta_{y} \leq 1
$$

onde $\eta_{x}$ e $\eta_{v}$, apresentados na seção 2.2 são os números de Courant. Para $a=2|u|_{\text {max }}$ e $b=2|v|_{\text {max }}$ determina-se a condição (5.24) e (5.25).

De acordo com (Tomé e McKee, 1994), (Griebel et al., 1998) e (Fortuna, 2000), um fator de segurança Fact, tal que Fact $\in(0,1]$, deve ser aplicado nas restrições (5.23), (5.24) e (5.25).

Finalmente, o valor de $\delta t$ utilizado na simulação, deve satisfazer simultaneamente as restrições (5.23), (5.24) e (5.25), e multiplicado pelo fator de segurança, ou seja

$$
\delta t=F a c t . \min \left(\delta t_{v i s c}, \delta t_{C F L x}, \delta t_{C F L y}\right)
$$

Outros autores que utilizam metodologias baseadas no método MAC, com natureza explícita da integração temporal, adotam outras expressões para o critério de estabilidade. Um exemplo, é a restrição descrita por (Casulli, 1981), que adota como restrição para o passo temporal

$$
\delta t \leq A\left[\frac{|u|_{\max }}{\delta x}+\frac{|v|_{\max }}{\delta y}+\frac{2 \mu}{\rho}\left(\frac{1}{(\delta x)^{2}}+\frac{1}{(\delta y)^{2}}\right)\right]^{-1},
$$

onde $A=\min (u, v, \rho)$.

\subsection{Método de Projeção-1 para Formulações Implí- citas na metodologia GENSMAC}

O primeiro método de projeção utilizado pelas Formulações Implícitas (FI) na metodologia GENSMAC é o método de projeção baseado em (Chorin, 1968) e conhecido como método de projeção sem-pressão, e aqui denotado como Projeção-1. Este método elimina o gradiente de pressão da equação (5.1). Na seção 4.3, foi apresentada uma técnica muito utilizada, que é discretizar no nível de tempo $n+1$ os termos viscosos 
na equação (5.1). Portanto, outra modificação nesta equação é a aplicação de métodos implícitos para os termos viscosos, tornando a equação (5.1) de natureza implícita na integração temporal. Os métodos implícitos utilizados foram os tradicionais Implícito Regressivo (IR) e Crank-Nicolson (CN). Com o objetivo de tentar melhorar a precisão temporal, um método Adams de passo múltiplo também foi utilizado. Este método utiliza o método CN para os termos viscosos e o método Adams-Bashforth (Lambert, 1973), que é uma técnica explícita, para os termos convectivos da equação (5.1). Este método é conhecido como Adams-Bashforth/Crank-Nicolson (AB/CN) (Peyret e Taylor, 1983), (Kim e Moin: 1985), (Chattopadhyay e Saha, 2003), (Denaro, 2003). Portanto a equação (5.1), na forma discreta no tempo, aplicando o método de Projeção-1 e utilizando as formulações implícitas, é reescrita da seguinte forma

- Projeção-1 - Método IR

$$
\frac{\partial \tilde{\mathbf{u}}}{\partial t}-\frac{1}{R e} \nabla^{2} \tilde{\mathbf{u}}=-\nabla \cdot(\mathbf{u u})^{n}+\frac{1}{F r^{2}} \mathbf{g}^{n}
$$

ou

$$
\tilde{\mathbf{u}}-\frac{\delta t}{\bar{K} e} \nabla^{2} \tilde{\mathbf{u}}=\mathbf{u}^{n}+\delta t\left\{-\nabla \cdot(\mathbf{u u})^{n}+\frac{1}{F r^{2}} \mathbf{g}^{n}\right\}
$$

- Projeção-1 - Método CN

$$
\frac{\partial \tilde{\mathbf{u}}}{\partial t}-\frac{1}{2 R e} \nabla^{2} \tilde{\mathbf{u}}=-\nabla \cdot(\mathbf{u} \mathbf{u})^{n}+\frac{1}{2 R e} \nabla^{2} \mathbf{u}^{n}+\frac{1}{F r^{2}} \mathrm{~g}^{n}
$$

ou

$$
\tilde{\mathbf{u}}-\frac{\delta t}{2 R \boldsymbol{e}} \nabla^{2} \tilde{\mathbf{u}}=\mathbf{u}^{n}+\delta t\left\{-\nabla \cdot(\mathbf{u u})^{n}+\frac{1}{2 R e} \nabla^{2} \mathbf{u}^{n}+\frac{1}{F r^{2}} \mathbf{g}^{n}\right\}
$$

- Projeção-1 - Método $A B / C N$

$$
\frac{\partial \tilde{\mathbf{u}}}{\partial t}-\frac{1}{2 R e} \nabla^{2} \tilde{\mathbf{u}}=-\frac{3}{2} \nabla \cdot(\mathbf{u u})^{n}+\frac{1}{2} \nabla \cdot(\mathbf{u u})^{n-1}+\frac{1}{2 R e} \nabla^{2} \mathbf{u}^{n}+\frac{1}{F r^{2}} \mathbf{g}^{n}
$$

ou

$$
\tilde{\mathbf{u}}-\frac{\delta t}{2 R e} \nabla^{2} \tilde{\mathbf{u}}=\mathbf{u}^{n}+\delta t\left\{-\frac{3}{2} \nabla \cdot(\mathbf{u u})^{n}+\frac{1}{2} \nabla \cdot(\mathbf{u u})^{n-1}+\frac{1}{2 R e} \nabla^{2} \mathbf{u}^{n}+\frac{1}{F r^{2}} \mathbf{g}^{n}\right\} .
$$


5.3 Método de Projeção-1 para Formulações Implícitas na metodologia GENSMAC 61

Utilizando a teoria dos métodos de projeção, tem-se que o campo de velocidade final é decomposto em um campo de velocidade intermediário $\tilde{\mathbf{u}}$ e o gradiente de um potencial escalar $\nabla \psi$. No método de Projeção-1, a função escalar $\psi$ é calculada em todo domínio, como será mostrado na próxima subseção.

\subsubsection{Condições Iniciais e de Contorno para o método de Proje- ção-1}

Utilizando o método de Projeção-1, as FI na metodologia GENSMAC, exigem que as condições iniciais para as variáveis primitivas velocidade e pressão também sejam prescritas no início dos cálculos, como no método GENSMAC.

As condições de contorno são análogas ao do método GENSMAC, exceto para as superfícies livres. As velocidades nas superfícies livres são fornecidas pela equação (5.15), mas a pressão não será calculada pela equação (5.14). Como no método de Projeçẫo- 1 o gradiente de pressão $\nabla \hat{p}$ é eliminado, $\tilde{p}=0$ em todo o domínio, inclusive nas superfícies livres. Portanto a equação (5.14) não é aplicada para $\tilde{p}$, mas será aplicada para o potencial escalar $\psi$, alterando mais uma vez a metodologia GENSMAC.

No método GENSMAC, a equação de Poisson (5.7) é valida em todo o domínio que contém fluido, com as condições de contorno descritas no Passo 3 na seção 5.2.

No método de Projeção-1 utilizando FI, além da equação de Poisson (5.7), uma nova equação é imposta no potencial $\psi$ para as superfícies livres. Essa nova equação é derivada da equação (5.14) com as velocidades implícitas, ou seja, as condições de contorno nas superfícies livres também são implícitas. Essa técnica foi aplicada, pois observou-se, que para problemas confinados (sem superfície livre), apenas a discretização dos termos viscosos no nível de tempo $n+1$ das equações descritas na seção 5.3 foi suficiente para se obter um método incondicionalmente estável. Mas o mesmo não ocorreu para problemas com superfícies livres, fazendo-se necessário, a aplicação de condições de contorno implícitas nas superfícies livres. Portanto a equação (5.14) é reescrita, na forma implícita e discreta no tempo como

$$
-p^{n+1}+\frac{2}{R e}\left[\frac{\partial u^{n+1}}{\partial x} n_{x}^{2}+\frac{\partial v^{n+1}}{\partial y} n_{y}^{2}+\left(\frac{\partial u^{n+1}}{\partial y}+\frac{\partial v^{n+1}}{\partial x}\right) n_{x} n_{y}\right]=0 .
$$


Para ilustrar a aplicação da equação (5.35) para o potencial escalar $\psi$ nas superfícies livres, considere os 4 casos onde a célula $S$ da superfície livre, está em contato com células E.

- Caso 1: Na configuração da figura 5.2 , o vetor $\mathbf{n}=(1,0)$ e a equação (5.35) é reduzida a

$$
p^{n+1}=\frac{\dot{z}}{\operatorname{Re}}\left(\frac{\partial u^{n+1}}{\partial x}\right)
$$

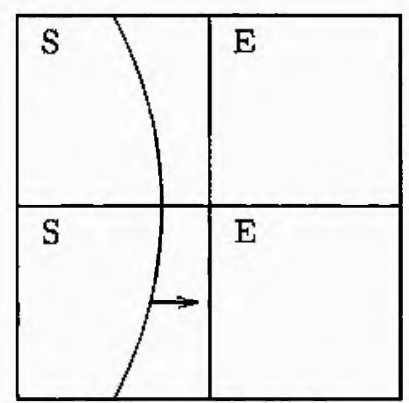

Figura 5.2: Configuração de uma célula computacional mostrando uma célula da superfície livre em contato, na sua face lateral direita, com uma célula vazia.

Da equação da continuidade (3.17) tem-se

$$
\frac{\partial u^{n+1}}{\partial x}=\frac{\partial v^{n+1}}{\partial y}
$$

que substituída em (5.36) produz

$$
p^{n+1}=-\frac{2}{R e}\left(\frac{\partial v^{n+1}}{\partial y}\right)
$$

Pela equação (5.6), o campo de velocidade final na direção $y$, discretizada no tempo é

$$
v^{n+1}=\tilde{v}-\frac{\partial \psi^{n+1}}{\partial y}
$$

Substituindo (5.39) em (5.38) define-se

$$
p^{n+1}=-\frac{2}{R} e\left(\frac{\partial \bar{v}}{\partial y}-\frac{\partial^{2} \psi^{n+1}}{\partial y^{2}}\right) .
$$


De acordo com o método de Projeção-1, $\bar{p}=0$ em todo o domínio, portanto a equação (5.13) é agora reescrita na forma discreta no tempo como

$$
p^{n+1}-\frac{\psi^{n+1}}{\delta i}
$$

Finalmente, após substituir (5.40) em (5.41), constroem-se a equação para o potencial escalar $\psi$ nas superfícies livres para o Caso 1, que é expressa pela equação

$$
\psi^{\pi+1}-\frac{2 \delta t}{R e}\left(\frac{\partial^{2} \psi^{n+1}}{\partial y^{2}}\right)=\cdot \frac{2 \delta t}{R e}\left(\frac{\partial \ddot{v}}{\partial y}\right)
$$

A equação (5.42) é utilizada também para se calcular o potencial $\psi$ nas superfícies livres para o caso em que o vetor $\mathbf{n}=(-1,0)$. Para isto, basta substituir $\mathbf{n}=$ $(-1,0)$ na equação $(5.35)$ e os cálculos são análogos.

- Caso 2: A figura 5.3 mostra o caso em que o vetor $\mathbf{n}=(0,1)$, e desta forma a equação (5.35) é escrita na forma

$$
p^{n+1}=\frac{2}{R e}\left(\frac{\partial v^{n+1}}{\partial y}\right)
$$

e pela equação (5.37), tem-se

$$
p^{n+1}=-\frac{2}{n E}\left(\frac{\partial_{n} n+1}{\partial i \bar{u}}\right)
$$

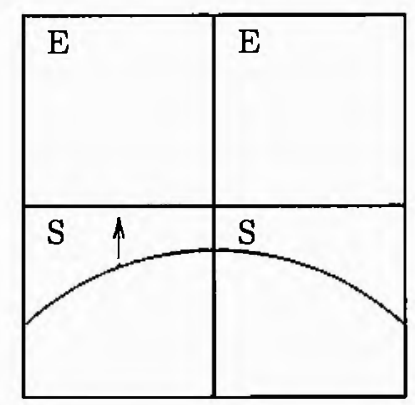

Figura 5.3: Configuração de uma célula computacional mostrando uma célula da superfície livre em contato, na sua face superior, com uma célula vazia. 
A equação (5.6) fornece a equação para o campo de velocidade final na direção $x$

$$
u^{n+1}=\tilde{u}-\frac{\partial \psi^{n+1}}{\partial x}
$$

Substituindo (5.45) em (5.44) produz

$$
{ }^{n+1}=-\frac{2}{R e}\left(\frac{\partial \tilde{u}}{\partial x}-\frac{\partial^{2} \psi^{n+1}}{\partial x^{2}}\right) .
$$

Utilizando a equação (5.41), pode se escrever a equação para $\psi$ nas superfícies livres para o Caso 2,

$$
\psi^{n+1}-\frac{2 \delta t}{R e}\left(\frac{\partial^{\tilde{z}} \psi^{n+1}}{\partial x^{2}}\right)=-\frac{2 \delta t}{R e}\left(\frac{\partial \tilde{u}}{\partial x}\right)
$$

No caso em que o vetor $\mathbf{n}=(0,-1)$, utiliza-se também (5.47) para o cálculo de $\psi$ nas superfícies livres.

- Caso 3: Neste caso, como pode-se ver na figura 5.4 , o vetor $\mathbf{n}=\left(\frac{\sqrt{2}}{2}, \frac{\sqrt{2}}{2}\right)$ e a equação (5.35), após aplicação da equação da continuidade (3.17), resume-se em

$$
p^{i-i}: 1=\frac{1}{R e}\left(\frac{\partial u^{n+1}}{\partial y}+\frac{\partial v^{n+1}}{\partial x}\right) \text {. }
$$

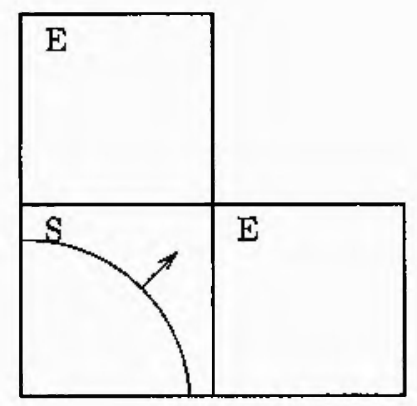

Figura 5.4: Configuração de uma célula computacional mostrando uma célula da superfície livre e que possui duas célula vazia adjacentes.

Substituindo as equações (5.39) e (5.45) na equação (5.48) tem-se

$$
p^{n+1}=\frac{1}{R e}\left(\frac{\partial \tilde{u}}{\partial y}-\frac{\partial^{2} \psi^{n+1}}{\partial x \partial y} \div \frac{\partial \tilde{v}}{\partial x}-\frac{\partial^{2} \psi^{n+1}}{\partial x \partial y}\right)
$$


Após substituir (5.41) em (5.49), a equação

$$
r_{r}^{n+1}+\frac{2 \delta t}{R e}\left(\frac{\partial^{2} \psi^{n+1}}{\partial x \partial y}\right)=\frac{\delta t}{R e}\left(\frac{\partial \tilde{u}}{\partial y}+\frac{\partial \tilde{v}}{\partial x}\right)
$$

é utilizada para se obter $\psi$ nas superfícies livres para o Caso 3 .

Para o vetor $\left.\mathbf{n}=i-\frac{\sqrt{\frac{2}{2}}}{\hat{4}},-\frac{\sqrt{\frac{\pi}{2}}}{\hat{a}}\right)$, a equação $(5.50)$ é utilizada para calcular $\psi$ nas superfícies livres.

- Caso 4: Pode-se ver na figura 5.5 uma configuração, onde o vetor $n=\left(\frac{\sqrt{2}}{2},-\frac{\sqrt{2}}{2}\right)$ e a equação (5.35) é reescrita como

$$
p^{n+1}=-\frac{1}{R e}\left(\frac{\partial u^{n+1}}{\partial y}+\frac{\partial v^{n+1}}{\partial x}\right)
$$

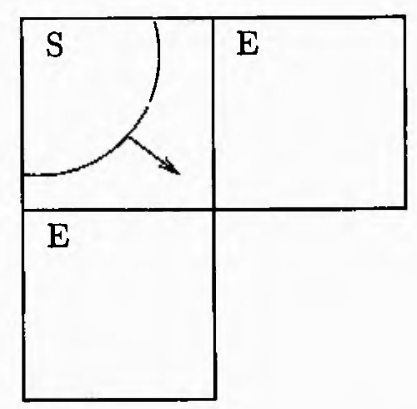

Figura 5.5: Configuração de uma célula computacional mostrando uma célula da superfície livre e que possui duas célula vazia adjacentes.

A substituição de (5.39) e (5.45) em (5.51) resulta na equação

$$
p^{n+1}=-\frac{1}{R}-\left(\frac{\partial \bar{u}}{\partial y}-\frac{\partial^{2} \psi^{n+1}}{\partial x \partial y} \div \frac{\partial \tilde{v}}{\partial x}-\frac{\partial^{2} \psi^{n+1}}{\partial x \partial y}\right)
$$

Mais uma vez, substituindo a equação (5.41) em (5.52), obtêm-se a equação para o cálculo de $\psi$ nas superfícies livres para o Caso 4, escrita da seguinte forma

$$
\psi^{n+1} \quad \frac{2 \delta t}{\operatorname{Re}}\left(\frac{\partial^{2} \psi^{n+1}}{\partial x \partial y}\right)=\frac{\delta t}{R e}\left(\frac{\partial \tilde{u}}{\partial y}+\frac{\partial \tilde{v}}{\partial x}\right)
$$

A equação (5.53) é aplicada também para se calcular $\psi$ nas superfícies livres no caso em que o vetor $\mathrm{n}=\left(-\frac{\sqrt{2}}{2}, \frac{\sqrt{2}}{2}\right)$. 
Após o cálculo do potencial escalar $\psi$ utilizando condições de contorno implícitas, como foi apresentado, o campo de velocidade final é atualizado pelas equações

$$
\begin{aligned}
& u^{n+1}=\bar{u}-\frac{\partial \psi^{n+1}}{\partial x} \\
& v^{n+1}=\tilde{v}-\frac{\partial \psi^{n+1}}{\partial y}
\end{aligned}
$$

Na metodologia GENSMAC, o campo de velocidade final (5.54) utiliza o potencial $\psi$ apenas no domínio que contém fluido, pois $\psi$ é calculado pela equação de Poisson (5.7) apenas neste domínio. No método de Projeção- 1 utilizando FI, o campo de velocidade final (5.54) utiliza $\psi$ para todo o domínio, isto é, para região de fluido e para as superfícies livres.

Nota-se que na metodologia GENSMAC, a equação que atualiza o campo de pressão é a equação (5.41) na região de fluido, pois neste caso, $\tilde{p}=0$, e para as superfícies livres, como $\psi=0$, a equação que atualiza a pressão é

$$
p^{n+1}=\bar{p},
$$

onde $\hat{p}$ é calculada por (5.14).

Entretanto, para o método de Projeção-1 utilizando FI, o campo de pressão é calculado pela equação (5.41) para todo domínio.

\subsubsection{Algoritmo do Método de Projeção-1 utilizando Formu- lações Implícitas}

Após o desenvolvimento do método de Projeção- 1 utilizando FI, pode-se escrever um algoritmo, com os passos interrelacionados. A seqüência de passos, assim como no algoritmo do método GENSMAC, consiste em atualizar as variáveis discretas, a partir de um tempo inicial $t_{n}$. O algoritmo é descrito como segue:

- Passo 1: Como neste método o gradiente de pressão $\nabla \tilde{p}$ é eliminado da formulação, neste passo, deve-se calcular as velocidades nas superfícies livres pela equação (5.15);

- Passo 2: Calcular um campo de velocidade intermediário $\tilde{\mathbf{u}}(\mathbf{x}, t)$ em $t=t_{n}+\delta t$. Se o método implícito escolhido para os termos viscosos for o IR, utiliza-se a 

equação (5.29), se for $\mathrm{CN}$ (5.31) e se a opção for o método $\mathrm{AB} / \mathrm{CN}$, então a equação utilizada é (5.33);

- Passo 3: Resolver a equação de Poisson (5.17) para o potencial $\psi$ na região contendo fluido, e nas superfícies livres, calcular $\psi$ através das equações descritas na subseção 5.3.1. As condições de contorno para a equação de Poisson (5.17) e para $\psi$ nas superfícies livres são:

- Condições homogêneas do tipo Neumann no contorno rígido, ou seja

$$
\frac{\partial \psi}{\partial n}=0
$$

Esta condição é utilizada também, nas regiões de entrada de fluido, caso existam.

- Condições homogéneas do tipo Dirichlet nas regiões de saída de fluido (caso existam), ou seja

$$
\psi=0
$$

- Passo 4: Atualizar o campo de velocidade final através da equação (5.18), repetida aqui

$$
\mathbf{u}^{n+1}=\tilde{\mathbf{u}}-\nabla \psi^{n+1}
$$

em todo o domínio;

- Passo 5: Atualizar o campo de pressão final pela equação (5.41);

- Passo 6: Determinar as novas posições das partículas marcadoras virtuais resolvendo as equações (5.21).

\subsubsection{Condição de Estabilidade do Método de Projeção-1 uti- lizando Formulações Implícitas}

Como foi visto na subseção 5.2.4, o tratamento explícito dos termos viscosos (4.30) e (4.31) da equação (5.16), impõem a restrição (5.22) sobre o passo temporal. Essa restrição, como mencionado anteriormente, se torna muito severa quando $R e<1$, ou seja, em problemas viscosos, o método explícito pode se tornar ineficiente. De acordo com (Pracht, 1971), essa ineficiência pode ser claramente observada, reescrevendo (5.22) 
na forma dimensional, ou seja

$$
\delta t \leq \frac{1}{2 \nu}\left(\frac{1}{(\delta x)^{2}}+\frac{1}{(\delta y)^{2}}\right)^{-1}
$$

e para simplificar, admite-se $\delta x=\delta y$, e desta forma, (5.56) resume em

$$
\delta t \leq \frac{(\delta x)^{2}}{4 \nu}
$$

De acordo com a definição de número de Reynolds (3.12), a equação (5.57) é reescrita

$$
\left(\frac{4 L}{\delta x}\right)\left(\frac{U \delta t}{\delta x}\right) \leq R e .
$$

Como apresentado na subseção 5.2.4, outra condição de estabilidade a ser imposta é (5.24), e dessa condição pode se escrever

$$
\frac{U \delta t}{\delta x} \approx \frac{1}{2}
$$

onde define-se $|u|_{\max }=U$.

Portanto, quando utiliza-se a condição (5.59) em (5.58), tem-se

$$
\frac{2 L}{\delta x}<R e \Rightarrow \delta x>\frac{2 L}{R e}
$$

A restrição (5.60), conforme (Pracht, 1971), é responsável por impor problemas de estabilidade quando $R e<1$ na utilização de métodos explícitos, pois os valores de $\delta x \mathrm{e}$ do parâmetro $L$ devem ser escolhidos com cuidado para que seja satisfeita essa restrição.

A aplicação de métodos implícitos nos termos viscosos, como foi feito nas equações (5.29), (5.31) e (5.33) pode eliminar em principio a restrição (5.22) e assim superar a restrição (5.60). Desta forma, as restrições sobre $\delta t$ para o método de Projeção-1 utilizando FI seriam imposta apenas pela condição CFL, isto é, pelas condições (5.24) e (5.25). Deve-se lembrar, como foi comentado na seção 4.3, que apesar das formulações implícitas permitirem um passo temporal maior que o permitido pelo método explícito, a solução numérica pode sofrer perda de precisão se $\delta t$ for muito grande. 
5.4 Método de Projeção-๕́ para Formulações Implícitas na metodologia GENSMAC 69

\subsection{Método de Projeção-2 para Formulações Implí- citas na metodologia GENSMAC}

Utilizando um método de projeção com acréscimo da pressão formulações implícitas foram aplicadas na metodologia GENSMAC. O acréscimo do gradiente de pressão na equação (5.1), modifica as equações para o cálculo do campo de velocidade intermediário do método Projeção-1. No presente trabalho, o método que conserva o gradiente de pressão será denotado como Projeção-2.

Da mesma forma que no método de Projeção-1, os termos viscosos foram discretizados implicitamente, transformando a equação (5.1) para o método Projeção-2 para a forma

- Projeção-2 - Método IR

$$
\frac{\partial \tilde{\mathbf{u}}}{\partial t}-\frac{1}{R e} \overline{\mathrm{v}}^{2} \tilde{\mathrm{u}}--\nabla \cdot(\mathbf{u} \mathbf{u})^{n}-\nabla \tilde{\mathrm{j}}+\frac{1}{F r^{2}} \mathbf{s}^{n}
$$

ou

$$
\tilde{\mathbf{u}}-\frac{\delta t}{R e} \nabla^{\prime} \overline{\mathbf{i}}=\mathbf{u}^{n}+\delta t\left\{-\nabla \cdot(\mathbf{u u})^{n}-\nabla \bar{p}+\frac{1}{\Gamma r^{2}} \mathbf{g}^{n}\right\}
$$

- Projeção-2 - Método CN

$$
\frac{\partial \tilde{\mathbf{u}}}{\partial t}-\frac{1}{2 R e} \nabla^{2} \tilde{\mathbf{u}}=-\nabla \cdot(\mathbf{u u})^{n}+\frac{1}{2 R e} \nabla^{2} \mathbf{u}^{n}-\nabla \tilde{p}+\frac{1}{F r^{2}} \mathbf{g}^{n}
$$

ou

$$
\tilde{\mathbf{u}}-\frac{\delta t}{2 R e} \nabla^{2} \tilde{\mathbf{u}}=\mathbf{u}^{n}+\delta t\left\{-\nabla \cdot(\mathbf{u u})^{n}+\frac{1}{2 \bar{R} e} \nabla^{2} \mathbf{u}^{n}-\nabla \tilde{p}+\frac{1}{F r^{2}} \mathbf{g}^{n}\right\} .
$$

- Projeção-2 - Método $A B / C N$

$$
\frac{\partial \tilde{\mathbf{u}}}{\partial t}-\frac{1}{\dot{z} \bar{F} e} \nabla^{2} \tilde{\mathbf{u}}=-\frac{3}{2} \nabla \cdot(\mathbf{u u})^{n}+\frac{1}{2} \nabla \cdot(\mathbf{u u})^{n-1}+\frac{1}{2 R e} \nabla^{2} \mathbf{u}^{n}-\nabla \tilde{p}+\frac{1}{\left.\Gamma_{\tilde{r}}\right)} \mathbf{g}^{n},
$$

ou

$$
\tilde{\mathbf{u}}-\frac{\delta t}{2 R e} \nabla^{2} \tilde{\mathbf{u}}=\mathbf{u}^{n}+\delta t\left\{-\frac{3}{2} \nabla \cdot(\mathbf{u u})^{n}+\frac{1}{2} \nabla \cdot(\mathbf{u u})^{n-1}+\frac{1}{2 R e} \nabla^{2} \mathbf{u}^{n}-\nabla \tilde{p}+\frac{1}{F^{3} r^{2}} \mathbf{g}^{n}\right\} .
$$


O desenvolvimento do método de Projeção-2 utilizando FI, é análogo ao método de Projeção-1, com a diferença que, agora $\tilde{p}$ não se anula e deverá ser calculada.

\subsubsection{Condições Iniciais e de Contorno para o método de Proje- ção-2}

As condições iniciais e de contorno, exceto nas superfícies livres, são análogas ao método de Projeção-1. No método de Projeção-2, as velocidades nas superfícies livres são fornecidas pela equação (5.15), e como na Projeção-1, a equação (5.35), é utilizada para calcular o potencial escalar $\psi$ nas superfícies livres, utilizando as condições de contorno implicitamente. Para ilustrar a aplicação da equação (5.35) para o potencial escalar $\psi$ nas superfícies livres no método de Projeção-2 utilizando FI, considere os 4 casos onde uma célula $S$ está em contato com células $E$.

- Caso 5: Na configuração da figura 5.6 , o vetor $\mathbf{n}=(-1,0)$.

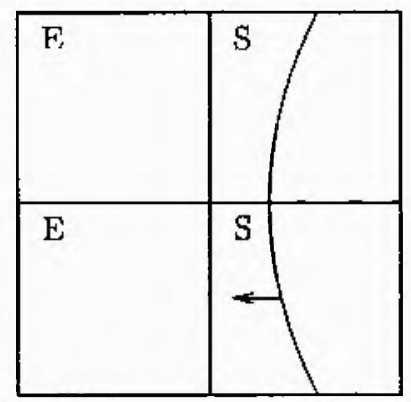

Figura 5.6: Configuração de uma célula computacional mostrando uma célula da superfície livre em contato, na sua face lateral esquerda, com uma célula vazia.

Os cálculos são análogos ao Caso 1 do método de Projeção-1, e portanto a equação para a pressão implícita para o Caso 5 é a mesma de (5.40).

No método de Projeção-2, a equação (5.19) atualiza o campo de pressão. Substituindo (5.40) em (5.19) tem-se

$$
\tilde{p}+\frac{\psi^{n+1}}{\delta t}=-\frac{2 \delta t}{R e} \cdot\left(\frac{\partial \tilde{v}}{\partial y}-\frac{\partial^{2} \psi^{n+1}}{\partial y^{2}}\right)
$$


Portanto, a equação para o potencial escalar $\psi$ nas superfícies livres é dada pela equação

$$
\psi^{n+1}-\frac{2 \delta t}{R e}\left(\frac{\partial^{2} \psi^{n+1}}{\partial y^{2}}\right)=-\frac{2 \delta t}{R e}\left(\frac{\partial \tilde{v}}{\partial y}\right)-\delta t \tilde{p}
$$

A equação (5.68) também é utilizada para calcular $\psi$ nas superfícies livres quando o vetor normal é $\mathbf{n}=(1,0)$.

- Caso 6: A figura 5.7 mostra o caso, em que o vetor $\mathbf{n}=(0,-1)$.

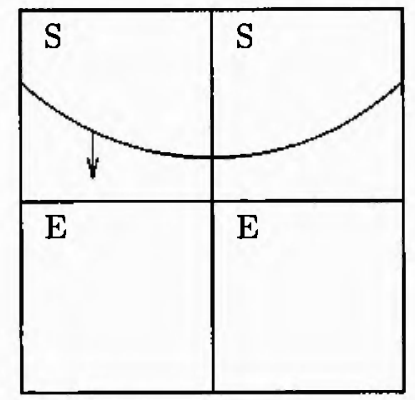

Figura 5.7: Configuração de uma célula computacional, mostrando uma célula da superfície livre em contato, na sua face inferior, com uma célula vazia.

Neste caso, para se obter a equação para a pressão implícita, pode-se repetir os cálculos feitos no Caso 2 do método de Projeção-1, obtendo a equação (5.46). De acordo com o método de Projeção-2 utilizando FI, ao substituir a equação (5.19) para a atualização da pressão, na equação (5.46), defini-se a equação para $\psi$ nas superfícies livres para o Caso 6 como

$$
\psi^{n+1}-\frac{2 \delta t}{R e}\left(\frac{\partial^{2} \psi^{n+1}}{\partial \tilde{x}^{2}}\right)=-\frac{2 \delta t}{R_{c}}\left(\frac{\partial \bar{u}}{\partial \bar{\partial}}\right)-\delta t \tilde{p}
$$

No caso em que $\mathbf{n}=(0,1)$, usa-se a mesma equação (5.69) para o calcular $\psi$ nas superfícies livres.

- Caso 7: Quando $\mathbf{n}=\left(-\frac{\sqrt{2}}{2},-\frac{\sqrt{2}}{2}\right)$, a configuração pode ser vista pela figura 5.8. Analogamente ao Caso 3 do método de Projeção-1, a equação para a pressão implícita é dada por (5.49), e substituindo essa equação em (5.19) tem-se

$$
\psi^{n+1}+\frac{2 \delta t}{R e}\left(\frac{\partial^{2} \psi^{n+1}}{\partial x \partial y}\right)=\frac{\delta t}{R e}\left(\frac{\partial \bar{u}}{\partial y}+\frac{\partial \tilde{v}}{\partial x}\right)-\hat{o t} \tilde{F}
$$




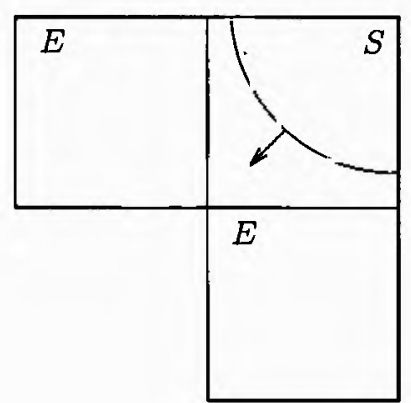

Figura 5.8: Configuração de uma célula computacional mostrando uma célula da superfície livre e que possui duas célula vazia adjacentes.

Portanto a equação (5.70) calcula $\psi$ nas superfícies livres para o Caso 7 e quando o vetor $\mathrm{n}=\left(\frac{\sqrt{2}}{2}, \frac{\sqrt{2}}{2}\right)$.

- Caso 8: Neste caso, como ilustra a figura 5.9 , o vetor $n=\left(-\frac{\sqrt{2}}{2}, \frac{\sqrt{2}}{2}\right)$ e a equação para a pressão a ser utilizada é (5.52), pois os cálculos para o método de Projeção-2 são análogos ao Projeção-1, para o Caso 4.

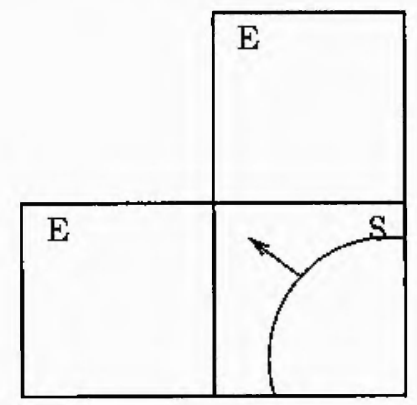

Figura 5.9: Configuração de uma célula computacional mostrando uma célula da superfície livre e que possui duas célula vazia adjacentes.

Substituindo (5.52) em (5.19), obtêm-se

$$
\dot{\psi}^{n+1}-\frac{2 \delta t}{R e}\left(\frac{\partial^{2} \psi^{n+1}}{\partial x \partial y}\right)=-\frac{\delta t}{R e}\left(\frac{\partial \tilde{u}}{\partial y}+\frac{\partial \bar{v}}{\partial x}\right)-\delta t \bar{p}
$$

Portanto, a equação (5.71) é aplicada para o Caso 8, quando deseja-se calcular $\psi$ nas superfícies livres utilizando o método Projeção-2 com FI. Para o vetor $\mathbf{n}=\left(\frac{\sqrt{2}}{2},-\frac{\sqrt{2}}{2}\right)$, deve se utilizar a mesma equação no cálculo de $\psi$. 
Da mesma forma que no método Projeção-1, o campo de velocidade final é calculado utilizando o potencial $\psi$. Portanto, a equação que calcula o campo final é (5.54).

A diferença mais expressiva entre os métodos Projeção-1 e Projeção-2 está na atualização da pressão. No método Projeção-2, a equação (5.19) é utilizada em todo o domínio, enquanto na Projeção-1, a equação para atualização do campo de pressão é (5.41).

\subsubsection{Algoritmo do Método de Projeção-2 utilizando Formu- lações Implícitas}

O algoritmo do método de Projeção-2 utilizando FI é descrito como segue:

- Passo 1: Neste método, como o gradiente de pressão é conservado, $\ddot{p}=p^{n}$, onde $p^{n}$ é a pressão calculada no tempo anterior pela equação (5.19). Calcular as velocidades nas superfícies livres pela equação (5.15);

- Passo 2: Conhecido um campo de pressão $\bar{p}$ em todo o domínio, isto é, na região de fluido e superfícies livres, calcular um campo de velocidade intermediário $\tilde{\mathbf{u}}(\mathbf{x}, t)$ em $t=t_{n}+\delta t$. Se o método implícito escolhido para os termos viscosos for o IR, utiliza-se a equação (5.61), se for CN utiliza-se a equação (5.63) ou (5.65) para $\mathrm{AB} / \mathrm{CN}$;

- Passo 3: Resolver a equação de Poisson (5.17) para o potencial $\psi$ na região contendo fluido, e nas superfícies livres, calcular $\psi$ através das equações da subseção 5.4.1. As condições de contorno para essas equações são as mesmas do Passo 3 do algoritmo do método de Projeção-1;

- Passo 4: Atualizar o campo de velocidade final como no Passo 4 do algoritmo do método de Projeção-1;

- Passo 5: Atualizar o campo de pressão final pela equação (5.19), repetida aqui

$$
p^{n+1}=\dot{p}+\frac{\psi^{n+1}}{\delta t}
$$

em todo domínio;

- Passo 6: Determinar as novas posições das partículas marcadoras virtuais resolvendo as equações (5.21). 


\subsubsection{Condição de Estabilidade do Método de Projeção-2 uti- lizando Formulações Implícitas}

A condição de estabilidade nesse caso, é a mesma obtida para o método de Projeção1 utilizando FI, ou seja, aplicando métodos implícitos nos termos viscosos nas equações (5.61), (5.63) ou (5.65), a restrição (5.22) pode ser superada. Para o método de Projeção-2 utilizando FI, conservando o gradiente de pressão, o que se espera, é uma solução numérica mais precisa do que no método de Projeção-1 para $\delta t$ relativamente grande.

\subsection{Método dos Gradientes Bi-conjugados para Sis-- temas Esparsos}

A aplicação dos métodos Projeção 1 e Projeção 2 para as Formulações Implícitas no GENSMAC, resulta em 3 sistemas lineares esparsos: 2 para as equações que calculam as velocidades intermediárias e 1 para o cálculo do potencial escalar $\psi$.

Quando se aplica as formulações implícitas, como o método Implícito Regressivo ou Crank-Nicolson, os termos viscosos são tratados de forma implícita, e desta maneira é necessário resolver os sistemas provenientes das velocidades $\bar{u}$ e $\tilde{v}$. Os sistemas lineares resultantes das equações (5.29), (5.31), (5.33), (5.61), (5.63) e (5.65) são esparsos, definidos positivos e simétricos. Devido à essas propriedades, um método iterativo eficiente é o método dos Gradientes Conjugados (GC) (Press et al., 1992), (Hackbush, 1994) e (Cuminato e Meneguette, 1999).

Na metodologia GENSMAC, o método GC é utilizado na resolução da equação de Poisson (5.7), pois o sistema resultante é esparso, definido positivo e simétrico. Mas como apresentado na seção anterior, os métodos Projeçä́o 1 e Projeção 2, resolvem além da equação (5.7), uma equação para o potencial $\psi$ nas superfícies livres, devido ao tratamento implícito das condições de contorno para a pressão. O sistema resultante dessas equações, possui matriz não simétrica, que por sua vez, não permite o uso do método GC. Portanto, a aplicação de um novo método iterativo para resolução de sistemas lineares é outra modificação feita na metodologia GENSMAC. 
O objetivo desta seção é apresentar o método dos Gradientes Bi-Conjugados (GBC), para solução de sistemas lineares

$$
A \cdot \mathbf{x}=\mathbf{b}
$$

com matriz $A$ não simétrica.

A formulação do método GBC, requer que inicialmente, construam-se 4 vetores $\mathbf{r}_{k}$, $\overline{\mathbf{r}}_{k}, \mathbf{p}_{k}$ e $\mathbf{p}_{k}, k=1,2, \ldots$. Deve-se fornecer os vetores iniciais $\mathbf{r}_{1}, \overline{\mathbf{r}}_{1}, \mathbf{p}_{1}=\mathbf{r}_{1}$ e $\overline{\mathbf{p}}_{1}=\overline{\mathbf{r}}_{1}$. Desta forma, pode-se definir o seguinte algoritmo

$$
\begin{aligned}
\alpha_{k} & =\frac{\mathbf{r}_{k}^{T} \cdot \overline{\mathbf{r}}_{k}}{\mathbf{p}_{k}^{T} \cdot \mathbf{A} \cdot \mathbf{p}_{k}} \\
\mathbf{r}_{k+1} & =\mathbf{r}_{k}-\alpha_{k} \mathbf{A} \cdot \mathbf{p}_{k} \\
\overline{\mathbf{r}}_{k+1} & =\overline{\mathbf{r}}_{k}-\alpha_{k} \mathbf{A}^{T} \cdot \overline{\mathbf{p}}_{k} \\
\hat{\rho}_{k} & =\frac{\overline{\mathbf{r}}_{k+1}^{T} \cdot \mathbf{r}_{k+1}}{\mathbf{r}_{k}^{T} \cdot \mathbf{r}_{k}} \\
\mathbf{p}_{k+1} & =\mathbf{r}_{k+1}+\beta_{k} \mathbf{p}_{k} \\
\mathbf{p}_{k+1} & =\overline{\mathbf{r}}_{k+1}+\beta_{k} \overline{\mathbf{p}}_{k}
\end{aligned}
$$

Os vetores dessa seqüência são bi-ortogonais, ou seja

$$
\overline{\mathbf{r}}_{i}^{T} \cdot \mathbf{r}_{j}=\mathbf{r}_{i}^{T} \cdot \overline{\mathbf{r}}_{j}=0, \quad j<i
$$

e também satisfazem a condição

$$
\overline{\mathbf{p}}_{\boldsymbol{i}}^{T} \cdot \mathbf{A} \cdot \mathbf{p}_{j}=\mathbf{p}_{i}^{T} \cdot \mathbf{A}^{T} \cdot \overline{\mathbf{p}}_{j}=0, \quad j<i
$$

e são reciprocamente ortogonais

$$
\overline{\mathbf{r}}_{i}^{T} \cdot \mathbf{p}_{j}=\mathbf{r}_{i}^{T} \cdot \mathbf{p}_{j}=0, \quad j<i
$$

A prova dessas propriedades é apresentada em (Fletcher, 1975). Portanto, para utilizar o algoritmo (5.73), um vetor inicial $\mathbf{x}_{1}$ é escolhido, e o resíduo inicial define o vetor $\mathbf{r}_{1}$,

$$
\mathbf{r}_{1}=\mathbf{b}-\mathbf{A} \cdot \mathbf{x}_{1}
$$


$\operatorname{com} \mathbf{r}_{1}=\mathbf{r}_{1}$. O próximo vetor da seqüência de aproximações é calculado por

$$
\mathbf{x}_{k+1}=\mathbf{x}_{k}+\alpha_{k} \mathbf{p}_{k}
$$

Um critério de parada para o método GBC é

$$
\frac{\left|\mathbf{A x} \mathbf{x}_{k+1}-\mathbf{b}\right|}{|\mathbf{b}|}<\text { tol }
$$

onde tol é uma tolerância escolhida de acordo com o problema.

Como o método GBC é mais eficiente se a matriz $\mathbf{A}$ for bem-condicionada (Quarteroni et al., 2000), pode-se utilizar um método de precondicionamento (Golub e Loan, 1989), isto é, o sistema linear (5.72) é transformado para

$$
\left(\tilde{\mathbf{A}}^{-1} \mathbf{A}\right) \mathbf{x}=\tilde{\mathbf{A}}^{-1} \mathbf{b}
$$

onde $\tilde{\mathbf{A}}$ é chamada matriz précondicionadora (Quarteroni et al., 2000) e o esquema utilizado é chamado método dos Gradientes Bi-conjugado Precondicionado (GBCP). Neste trabalho, $\tilde{\mathbf{A}}$ foi tomada como a diagonal de $\mathbf{A}$.

Para se utilizar um precondicionamento, os vetores $\mathbf{z}_{k}$ e $\overline{\mathbf{z}}_{k}$ definidos como

$$
\tilde{\mathbf{A}} \mathbf{z}_{k}=\mathbf{r}_{k} \quad \text { e } \quad \tilde{\mathbf{A}}^{T} \overrightarrow{\mathbf{z}}_{k}=\overline{\mathbf{r}}_{k}
$$

são introduzidos no algoritmo (5.73). Note que as equações (5.81) representam sistemas lineares que devem ser resolvidos a todo passo. Desta forma a matriz $\tilde{\mathbf{A}}$ deve ser escolhida de forma que esses sistemas tenham soluções simples.

Portanto, a definição dos valores $\alpha_{k}$ e $\beta_{k}$ e dos vetores $\mathbf{p}_{k}$ e $\overline{\mathbf{p}}_{k}$ serão modificados para

$$
\begin{aligned}
\alpha_{k} & =\frac{\mathbf{z}_{k}^{T} \cdot \overline{\mathbf{z}}_{k}}{\overline{\mathbf{p}}_{k}^{T^{\prime}} \cdot \mathbf{A} \cdot \mathbf{p}_{k}} \\
\beta_{k} & =\frac{\ddot{\mathbf{r}}_{k+1}^{T} \cdot \mathbf{z}_{k+1}}{\mathbf{r}_{k}^{T} \cdot \mathbf{z}_{k}} \\
\mathbf{p}_{k+1} & =\mathbf{z}_{k+1}+\beta_{k} \mathbf{p}_{k} \\
\overline{\mathbf{p}}_{k+1} & =\overline{\mathbf{z}}_{k+1}+\beta_{k} \overline{\mathbf{p}}_{k} .
\end{aligned}
$$


O critério de parada do algoritmo (5.82) para o método GBCP pode ser expresso como

$$
\frac{\left|\tilde{A}^{-1} \cdot(\mathrm{Ax}-\dot{\mathrm{D}})\right|}{\left|\tilde{\mathrm{A}}^{-1} \cdot \mathrm{b}\right|}<\text { tol. }
$$

Atualmente o método dos GC vem sendo utilizado na metodologia GENSMAC com muito sucesso, atingindo convergência, na maioria dos problemas, com apenas algumas iterações. Esse sucesso pode ser justificado pelo fato que a condição inicial, do passo anterior, é muito boa, pois o passo temporal é muito pequeno. Os resultados sobre a convergência do método GC podem ser vistos em (Luenberger, 1984), (Golub e Loan, 1989), (Cuminato e Meneguette, 1999) e (Cunha, 2000).

Portanto existe uma grande resistência em substituir o método GC por qualquer outro. Mas como o sistema de equações resultante para o potencial $\psi$ utilizando os métodos Projeção-1 e Projeção-2 produz uma matriz não simétrica foi necessário introduzir um novo método iterativo, o método GBC. Porém, não existem resultados que garantam sua convergência, como no caso do método GC. Desta forma o método GBC pode falhar em alguns problemas. Detalhes sobre o método GBC e GBCP podem ser encontrado em (Lanczos, 1952), (Watson, 1976), (Vorst, 1992), (Press et al., 1992) e (Ayachour, 2003).

Além do método GBC, existem outras alternativas recomendadas na literatura para problemas esparsos: os métodos GMRES (Saad e Schltz, 1986), (Ayachour, 2003), QMR (Freund e Nachtigal, 1991), MINRES (Paige e Saunders, 1975), FGMRES (Saad, 1993), CMRH (Sadok, 1999), entre outros. Entretanto, parece não haver consenso de um método que seja claramente melhor. 


\section{Discretização das Equações e Ambiente FreeFlow2D}

\subsection{Introdução}

As etapas para a obtenção da solução numérica de um problema de escoamento de fluidos pode seguir a seguinte seqüência: dado um problema físico, utiliza-se modelagem matemática através de equações governantes e em seguida aplica-se um processo de discretização, obtendo uma solução aproximada. Após obter essa solução são feitas análises e interpretações dos resultados numéricos. Para interpretação dos resultados numéricos, muitas vezes usam-se técnicas de visualização. A visualização dos dados do escoamento, em diversos casos, permite que uma análise qualitativa rápida e direta seja feita. Portanto, na DFC, um processo muito importante e fundamental, é escolher de forma apropriada a técnica de resolução das equações governantes e um ambiente de simulação. Esse ambiente, deve unir técnicas de modelagem do problema, resolução das equações e visualização dos resultados. No presente trabalho, utiliza-se a sigla GENSMAC, já mencionado anteriormente, para o método de resolução das equações de Navier-Stokes, e utiliza-se também a sigla FreeFlow2D para o ambiente de simulação que implementa o método GENSMAC.

Para resolver numericamente as equações (3.15) e (3.16), como foi visto, utilizamse formulações implícitas na metodologia GENSMAC. A malha utilizada é a malha 
deslocada ou diferenciada ("staggered grid") de Harlow e Welch (Harlow e Welch, 1965), como mostra a figura 4.1. Nesse tipo de malha, a indexação das incógnitas na célula, como a pressão (ou outra componente $\phi$ do fluido), segue a mesma indexação da célula, e as velocidades, têm indexação fracionária. A técnica de discretização utilizada para resolver o conjunto de equações (3.15) e (3.16) é o método de diferenças finitas.

O ambiente FreeFlow2D, é um ambiente integrado para a simulação de escoamentos bidimensionais incompressíveis com superfícies livres. Esse sistema é composto dos módulos: de modelagem (Modelador), de simulação que utiliza a metodologia GENSMAC (Simulador), de visualização (Visualizador) e um módulo para reiniciar a simulação (Re-inicializador).

A seguir, as equações dos métodos de Projeção-1 e Projeção-2 utilizando Formulações Implícitas na forma discreta e o ambiente FreeFlow2D utilizado neste trabalho serão apresentadas.

\subsection{Discretização das Equações}

Quando deseja-se fazer um tratamento computacional de um modelo é necessário expressar de forma adequada as equações e o domínio em que elas são válidas. Logo utiliza-se o processo de discretização, que é dividido em duas fases:

1. Discretização de um domínio: A solução numérica das EDPs não pode ser obtida sobre um domínio contínuo devido aos infinitos pontos que o constituem. Logo, faz-se uma representação numérica dessa região, incluindo as posições dos pontos onde deseja-se aproximar a solução. Assim, o domínio é discretizado, ou seja, é dividido em número finito de regiões discretas, que são chamadas de células computacionais, como mostra a figura 6.1. Quando o problema é transiente, o intervalo de tempo é dividido em passo temporais de tamanho $\delta t$.

2. Discretização das equações: Na segunda fase, os termos que compõem as equações são escritos em função dos valores das incógnitas em pontos discretos adjacentes. A discretização das equações, fornece equações de diferenças relacionadas às variáveis dependentes nos pontos do domínio discretizado. Nessa fase, introduzem-se as condições de contorno do problema, normalmente modificandose as equações em pontos próximos da fronteira. 

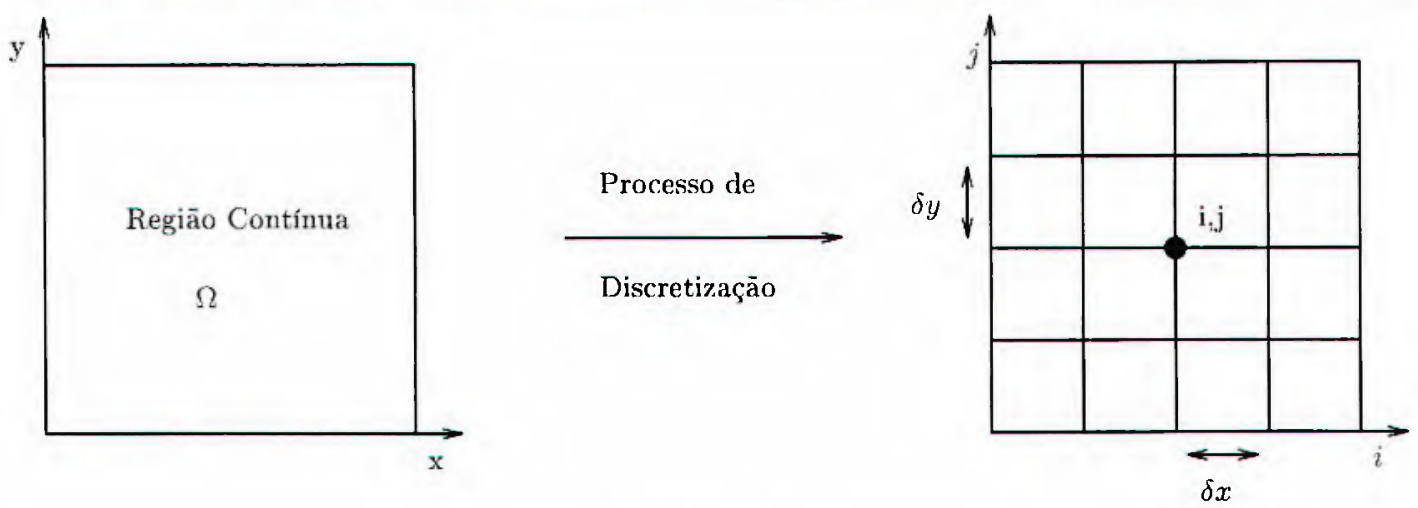

Figura 6.1: Processo de Discretização.

No presente trabalho, os termos viscosos das equações (5.30), (5.32) e (5.34) do método de Projeção-1, e as equações (5.62), (5.64) e (5.66) do método de Projeção2 são tratados de forma implícita e são aproximados por diferenças centrais de segunda ordem. A derivada temporal, dessas equações, é aproximada pelo método de Euler explícito, enquanto que o gradiente de pressão, presente apenas nas equações (5.62), (5.64) e (5.66), é aproximado por diferenças centrais. Os termos convectivos das equações $(5.30),(5.32),(5.34),(5.62),(5.64)$ e (5.66), denotados aqui como

$$
\begin{aligned}
& \operatorname{CONV}(u)=\frac{\partial u^{2}}{\partial x}+\frac{\partial(u v)}{\partial y} \\
& \operatorname{CONV}(v)=\frac{\partial(u v)}{\partial x}+\frac{\partial v^{2}}{\partial y}
\end{aligned}
$$

são aproximados por esquemas monotônicos e anti-difusivos (Ferreira, 2001). A equação de Poisson, é aproximada pelo operador Laplaciano de cinco pontos, e as equações para o potencial $\psi$ nas superfícies livres são discretizadas conforme os casos.

\subsubsection{Discretização das Equações de Conservação}

A discretização das equações de conservação será feita sobre uma malha diferenciada (como mostra a figura 4.1). A equação de conservação de movimento na direção $x$ é discretizada sobre a posição $\left(i+\frac{i}{2}, 1\right)$, enquanto que na direção y é discretizada sobre a 
posição $\left(i, j+\frac{1}{2}\right)$. Por fim, a equação da conservação de massa é discretizada no centro da célula, no ponto $(i, j)$.

Portanto, as equações de conservação no caso bidimensional, na forma discretas são dadas por:

\section{- Equação de Conservação de Movimento:}

- Método de Projeção-1 - Método IR

A equação (5.30) na direção $x$ é dada por

$$
\begin{aligned}
& \tilde{u}_{i+\frac{1}{2}, j}^{n+1}-\frac{\delta t}{R e}\left\{\frac{\tilde{u}_{i+\frac{3}{2}, j}-2 \tilde{u}_{i+\frac{1}{2}, j}+\tilde{u}_{i-\frac{1}{2}, j}}{\delta x^{2}}+\frac{\tilde{u}_{i+\frac{1}{2}, j+1}-2 \tilde{u}_{i+\frac{1}{2}, j}+\tilde{u}_{i+\frac{1}{2}, j-1}}{\delta y^{2}}\right\}^{n+1}= \\
& u_{i+\frac{1}{2}, j}^{n}+\delta t\left\{-\left.\operatorname{CONV}(u)\right|_{i+\frac{1}{2}, j}+\frac{1}{F r^{2}} g_{x}\right\}^{n},
\end{aligned}
$$

e na direção $y$,

$$
\begin{aligned}
& \tilde{v}_{i, j+\frac{1}{2}}^{n+1}-\frac{\delta t}{R e}\left\{\frac{\tilde{v}_{i+1, j+\frac{1}{2}}-2 \bar{v}_{i, j+\frac{1}{2}, j}+\tilde{v}_{i-1, j+\frac{1}{2}}}{\delta x^{2}}+\frac{\tilde{v}_{i, j+\frac{3}{2}}-2 \tilde{v}_{i, j+\frac{1}{2}}+\tilde{v}_{i, j-\frac{1}{2}}}{\delta y^{2}}\right\}^{n+1}= \\
& v_{i, j+\frac{1}{2}}^{n}+\delta t\left\{-\left.\operatorname{CONV}(v)\right|_{i, j+\frac{1}{2}}+\frac{1}{F r^{2}} g_{y}\right\}^{n} .
\end{aligned}
$$

- Método de Projeção-1 - Método CN

A equação (5.32) é discretizada na direção $x$ como

$$
\begin{aligned}
& \tilde{u}_{i+\frac{1}{2}, j}^{n+1}-\frac{\delta t}{2 R e}\left\{\frac{u_{i+\frac{3}{2}, j}-2 u_{i+\frac{1}{n}, j}+\tilde{u}_{i-\frac{1}{2}, j}}{\delta x^{2}}+\frac{\tilde{u}_{i+\frac{1}{2}, j+1}-2 u_{i \frac{1}{2}, j}+\tilde{u}_{i+\frac{1}{2}, j-1}}{\delta y^{2}}\right\}^{n+1}= \\
& u_{i+\frac{1}{2}, j}^{n}+\delta t\left\{\frac{1}{2 R e}\left(\frac{u_{i+\frac{3}{2}, j}-2 u_{i+\frac{1}{2}, j}+u_{i-\frac{1}{2}, j}}{\delta x^{2}}+\frac{u_{i+\frac{1}{2}, j+1}-2 u_{i+\frac{1}{2}, j}+u_{i+\frac{1}{2}, j-1}}{\delta y^{2}}\right)\right. \\
& \left.-\left.\operatorname{CONV}(u)\right|_{i+\frac{1}{2}, j}+\frac{1}{F^{2} r^{2}} g_{x}\right\}^{n},
\end{aligned}
$$


e na direção $y$,

$$
\begin{aligned}
& \tilde{v}_{i, j+\frac{1}{2}}^{n+1}-\frac{\delta t}{2 R e}\left\{\frac{\tilde{v}_{i+1, j+\frac{1}{2}}-2 \tilde{v}_{i, j+\frac{1}{2}}+\bar{v}_{i-1, j+\frac{1}{2}}}{\delta x^{2}}+\frac{\tilde{v}_{i, j+\frac{3}{2}}-2 \tilde{v}_{i, j+\frac{1}{2}}+\bar{v}_{i, j-\frac{1}{2}}}{\delta y^{2}}\right\}^{n+1}= \\
& v_{i, j+\frac{1}{2}}^{n}: \delta_{t}\left\{\frac{1}{2 R e}\left(\frac{v_{i+1, j+\frac{1}{2}}-2 v_{i, j+\frac{1}{2}}+v_{i-1, j+\frac{1}{2}}}{\delta x^{2}}+\frac{v_{i, j+\frac{3}{2}}-2 v_{i, j+\frac{1}{2}}+v_{i, j-\frac{1}{2}}}{\delta y^{2}}\right)\right. \\
& \left.-\left.\operatorname{CONV}(v)\right|_{i, j+\frac{1}{2}}+\frac{1}{F r^{2}} g_{y}\right\}^{n} .
\end{aligned}
$$

- Método de Projeção-1 - Método AB/CN

A equação (5.34) é discretizada na direção $x$ como

$$
\begin{aligned}
& \ddot{u}_{i+\frac{1}{2}, j}^{n+1}-\frac{\delta t}{2 R e}\left\{\frac{\tilde{u}_{i+\frac{3}{2}, j}-2 \tilde{u}_{i+\frac{1}{2}, j}+\bar{u}_{i-\frac{1}{2}, j}}{\delta x^{2}} ; \frac{\tilde{u}_{i+\frac{1}{2}, j+1}-2 \tilde{u}_{i+\frac{1}{2}, j}+\tilde{u}_{i+\frac{1}{2}, j-1}}{\partial y^{2}}\right\}^{n+1}= \\
& u_{i+\frac{1}{2}, j}^{n}+\delta t\left\{\frac{1}{2 R e}\left(\frac{u_{i+\frac{3}{2}, j}-2 u_{i+\frac{1}{2}, j}+u_{i-\frac{1}{2}, j}}{\delta x^{2}}+\frac{u_{i+\frac{1}{2}, i+1}-2 u_{i+\frac{1}{2}, j}+u_{i+\frac{1}{2}, j-1}}{\delta y^{2}}\right)\right. \\
& \left.--\left.\frac{3}{2} \operatorname{CONV}(u)\right|_{i+\frac{1}{2}, j}+\frac{1}{F r^{2}} g_{x}\right\}^{n}+\delta t\left\{\left.\frac{1}{2} \operatorname{CONV}(u)\right|_{i+\frac{1}{2}, j}\right\}^{n-1},
\end{aligned}
$$

e na direção $y$,

$$
\begin{aligned}
& \tilde{v}_{i, j+\frac{1}{2}}^{n+1}-\frac{\delta t}{2 R e}\left\{\frac{\bar{v}_{i+1, j+\frac{1}{2}}-2 \tilde{v}_{i, j+\frac{1}{2}}+\tilde{v}_{i-1, j+\frac{1}{2}}}{\delta x^{2}}: \frac{\bar{v}_{i, j+\frac{3}{2}}-2 \tilde{v}_{i, j+\frac{1}{2}}+\tilde{v}_{i, j-\frac{1}{2}}}{\delta y^{2}}\right\}^{n+1}= \\
& v_{i, j+\frac{1}{2}}^{n}-v_{i} i \frac{1}{2 R e}\left(\frac{v_{i+1, j+\frac{1}{2}}-2 v_{i, j ! \frac{1}{2}}+v_{i-1, j+\frac{1}{2}}}{\delta x^{2}}+\frac{v_{i, j+\frac{3}{2}}-2 v_{i, j+\frac{1}{2}}+v_{i, j-\frac{1}{2}}}{\delta y^{2}}\right) \\
& \left.-\left.\frac{3}{2} C O N V(v)\right|_{i, j+\frac{1}{2}}+\frac{1}{F r^{2}} g_{y}\right\}^{n}+\delta t\left\{\left.\frac{1}{2} \operatorname{CONV}(v)\right|_{i, j+\frac{1}{2}}\right\}^{n-1} .
\end{aligned}
$$

\section{- Método de Projeção-2 - Método IR}

A equação (5.62) na direção $x$ é discretizada como

$$
\begin{aligned}
& \tilde{u}_{i+\frac{1}{2}, j}^{n+1}-\frac{\delta t}{R e}\left\{\frac{\tilde{u}_{i+\frac{3}{2}, i}-2 u_{i+\frac{1}{2}, j}+\tilde{u}_{i-\frac{1}{2}, j}}{\delta x^{2}}+\frac{\tilde{u}_{i+\frac{1}{2}, j+1}-2 u_{i ! \frac{1}{2}, j}+\bar{u}_{i+\frac{1}{2}, j-1}}{\delta y^{2}}\right\}^{n+1}= \\
& u_{i+\frac{1}{2}, j}^{n}+\delta t\left\{-\left.\operatorname{CONV}(u)\right|_{i+\frac{1}{2}, j}-\frac{\left(\tilde{p}_{i+1, j}-\tilde{p}_{i, j}\right)}{\delta \varepsilon}+\frac{1}{F r^{2}} g_{x}\right\}^{n},
\end{aligned}
$$


e na direção $y$,

$$
\begin{aligned}
& \tilde{v}_{i, j+\frac{1}{2}}^{n+1}-\frac{\delta t}{R e}\left\{\frac{\ddot{v}_{i+1 . i+\frac{1}{2}}-\bar{z} \ddot{v}_{i, j+\frac{1}{2}}+\tilde{v}_{i-1, j+\frac{1}{2}}}{\delta x^{2}}: \frac{\tilde{v}_{i, j+\frac{3}{2}}-2 \tilde{v}_{i, j+\frac{1}{2}}+\tilde{v}_{i, j-\frac{1}{2}}}{\delta y^{2}}\right\}^{n+1}= \\
& v_{i, j+\frac{1}{2}}^{n}+\delta t\left\{-\left.\operatorname{CONV}(v)\right|_{i, j+\frac{1}{2}}-\frac{\left(\tilde{n}_{i, j ! 1}-\tilde{n}_{t, \hat{w}}\right)}{\partial y}+\frac{1}{F_{i^{2}}^{2}} g_{y}\right\}^{n} \text {. }
\end{aligned}
$$

- Método de Projeção-2 - Método CN

A equação (5.64) na direção $x$ é dada por

$$
\begin{aligned}
& \tilde{u}_{i+\frac{1}{2}, j}^{n+1}-\frac{\delta t}{2 R e}\left\{\frac{\tilde{u}_{i+\frac{3}{2}, j}-2 \tilde{u}_{i+\frac{1}{2}, j}+\tilde{u}_{i-\frac{1}{2}, j}}{\delta x^{2}} \div \frac{\tilde{u}_{i+\frac{1}{2}, j+1}-2 \tilde{u}_{i+\frac{1}{2}, j}+\tilde{u}_{i+\frac{1}{2}, j-1}}{\delta y^{2}}\right\}^{n+1}= \\
& u_{i+\frac{1}{2}, j}^{n}+\delta i\left\{\frac{1}{2 R e}\left(\frac{u_{i+\frac{3}{2}, j}-2 u_{i+\frac{1}{2}, j}+u_{i-\frac{1}{2}, j}}{\delta x^{2}}+\frac{u_{i+\frac{1}{2}, j+1}-2 u_{i+\frac{1}{2}, j}+u_{i+\frac{1}{2}, j-1}}{\delta y^{2}}\right)\right. \\
& \left.-\left.\operatorname{CONV}(u)\right|_{i+\frac{1}{2}, j}-\frac{\left(\tilde{p}_{i+1, j}-\tilde{p}_{i, j}\right)}{\delta x}+\frac{1}{F r^{2}} g_{x}\right\}^{n},
\end{aligned}
$$

e na direção $y$,

$$
\begin{aligned}
& \tilde{v}_{i, j+\frac{1}{2}}^{n+1}-\frac{\delta t}{2 R e}\left\{\frac{\bar{v}_{i+1, j+\frac{1}{2}}-2 \tilde{v}_{i, j+\frac{1}{2}}+\bar{v}_{i-1, j+\frac{1}{2}}}{\delta x^{2}}+\frac{\tilde{v}_{i, j+\frac{3}{2}}-2 \tilde{v}_{i, j+\frac{1}{2}}+\tilde{v}_{i, j-\frac{1}{2}}}{\delta y^{2}}\right\}^{n+1}= \\
& v_{i, j+\frac{1}{2}}^{n}+\delta^{\frac{1}{v}}\left\{\frac{1}{2 R e}\left(\frac{v_{i+1, j+\frac{1}{2}}-2 v_{i, j+\frac{1}{2}}+v_{i-1, j+\frac{1}{2}}}{\delta x^{2}}+\frac{v_{i, j+\frac{3}{2}}-2 v_{i, j+\frac{1}{2}}+v_{i, j-\frac{1}{2}}}{\delta y^{2}}\right)\right. \\
& \left.-\left.\operatorname{CONV}(v)\right|_{i, j+\frac{1}{2}}-\frac{\left(\tilde{p}_{i, j+1}-\tilde{p}_{i, j}\right)}{\delta y}+\frac{1}{F r^{2}} g_{y}\right\}^{n} .
\end{aligned}
$$

- Método de Projeção-2 - Método $A B / C N$

A equação (5.34) é discretizada na direção $x$ como

$$
\begin{aligned}
& \tilde{u}_{i+\frac{1}{2}, j}^{n+1}-\frac{\delta t}{2 R e}\left\{\frac{\tilde{u}_{i+\frac{3}{2}, j}-2 \tilde{u}_{i+\frac{1}{2}, j}+\tilde{u}_{i-\frac{1}{2}, j}}{\delta x^{2}}+\frac{\tilde{u}_{i+\frac{1}{2}, j+1}-2 \tilde{u}_{i+\frac{1}{2}, j}+\tilde{u}_{i+\frac{1}{2}, j-1}}{\delta y^{2}}\right\}^{n+1}= \\
& u_{i+\frac{1}{2}, j}^{n}+\delta t\left\{\frac{1}{2 R e}\left(\frac{u_{i+\frac{3}{2}, j}-2 u_{i+\frac{1}{2}, j}+u_{i-\frac{1}{2}, j}}{\delta x^{2}}+\frac{u_{i+\frac{1}{2}, j+1}-2 u_{i+\frac{1}{2}, j}+u_{i+\frac{1}{n}, j-1}}{\delta y^{2}}\right)\right. \\
& \left.-\left.\frac{3}{2} \operatorname{CONV}(u)\right|_{i+\frac{1}{2}, j}-\frac{\left(\tilde{n}_{i, i, j}-\tilde{n}_{i, j}\right)}{\delta x}+\frac{1}{F r^{2}} g_{x}\right\}^{n}+\delta t\left\{\left.\frac{1}{2} \operatorname{CONV}(u)\right|_{i+\frac{1}{2}, j}\right\}^{n-1},
\end{aligned}
$$


e na direção $y$,

$$
\begin{aligned}
& i_{i, j+\frac{1}{2}}^{n+1}-\frac{\delta t}{2 R e}\left\{\frac{\tilde{v}_{i+1, j+\frac{1}{2}}-\bar{z} \bar{v}_{i, j i \frac{1}{2}}+\bar{v}_{i-1, j+\frac{1}{2}}}{\delta x^{2}} ; \frac{\left.\tilde{v}_{i, j+\frac{3}{2}}-2 \tilde{v}_{i, j+\frac{1}{2}}+\tilde{v}_{i, j-\frac{1}{2}}\right\}^{n+1}=}{\delta y^{2}}=\right. \\
& v_{i, j+\frac{1}{2}}^{n}+\delta t\left\{\frac{1}{2 R e}\left(\frac{v_{i+1, j+\frac{1}{2}}-2 v_{i, j+\frac{1}{2}}+v_{i-1, j+\frac{1}{2}}}{\delta x^{2}}+\frac{v_{i, j+\frac{3}{n}}-2 v_{i, j+\frac{1}{2}}+v_{i, j} \frac{1}{2}}{\delta y^{2}}\right)\right. \\
& \left.-\left.\frac{3}{2} \operatorname{CONV}(v)\right|_{i, j+\frac{1}{2}}-\frac{\left(\tilde{p}_{i, j+1}-\tilde{p}_{i, j}\right)}{\delta y}+\frac{1}{F r^{2}} g_{y}\right\}^{n}+\delta t\left\{\left.\frac{1}{2} \operatorname{CONV}(v)\right|_{i, j+\frac{1}{2}}\right\}^{n-1} .
\end{aligned}
$$

- Equação de Conservação de Massa ou Equação da Continuidade:

$$
\frac{\left(u_{i+\frac{1}{2}, j}-u_{i-\frac{1}{2}, j}\right)}{\delta x}+\frac{\left(v_{i, j+\frac{1}{2}}-v_{i, j-\frac{1}{2}}\right)}{\delta y}=0 .
$$

\subsubsection{Discretização dos Termos Convectivos}

Um desafio na Dinâmica de Fluidos Computacional é encontrar aproximações para o termo convectivo que não introduzam distorções na solução numérica. As principais aproximações freqüentemente utilizadas e mais conhecidas são:

- FOU ( First Order Upwind ) (Courant et al., 1952);

- CD (Price et al., 1966);

- SOU ( Second Order Upwind) (Fromm, 1968);

- HYBRID (Spalding, 1972);

- QUICK ( Quadratic Upstream Interpolation for Convective Kinematics ) (Leonard, 1979);

- PLDS ( Power-Law Differencing Schemes) (Patankar, 1980);

- SMART (Gaskell e Lau, 1988);

- HLPA ( Hybrid Linear/Parabolic Approximation ) (Zhu, 1992);

- VONOS ( Variable Order Non-Oscilatory Scheme) (Varonos e Bergeles, 1998). 
Para o leitor interessado nos fenômenos ocorridos com o uso de alguns desses métodos, pesquisadores como Raithby (Raithby, 1976), Ferreira (Ferreira, 2001) entre muitos outros, têm dedicado atenção especial em como tratar esses termos convectivos.

Como o objetivo principal deste trabalho é simular problemas viscosos para fluidos newtonianos, os termos convectivos não têm forte influência no escoamento. Mesmo assim, foram realizados testes com alguns desses métodos, em especial o método VONOS. Mais detalhes sobre o método VONOS e outras aplicações sobre termos convectivos, pode ser visto em (Ferreira, 2001).

\subsubsection{Discretização das Equações para o Potencial $\psi$}

Como discutido nas seções 5.3 e 5.4, para calcular o potencial $\psi$, utilizando os métodos Projeção-1 e Projeção-2 com FI, deve-se resolver a equação de Poisson (5.7) na região contendo fluido, e nas superfícies livres, novas equações foram introduzidas. As equações para o cálculo do potencial $\psi$ são discretizadas da seguinte forma:

\section{- Equação de Poisson:}

A equação de Poisson (5.7) é discretizada como

$$
\begin{aligned}
& \frac{\left(\psi_{i+1, j}^{n+1}-2 \psi_{i, j}^{n+i}+\psi_{i-1, j}^{n}\right)}{\delta x^{2}}+\frac{\left(\psi_{i, j+1}^{n+1}-2 \psi_{i, j}^{n+i}+\psi_{i, j-1}^{n+i}\right)}{\delta y^{2}}- \\
& \frac{\left(\bar{u}_{i+\frac{1}{2}, j}^{n+1}-\bar{u}_{i-\frac{1}{2}, j}\right)}{\delta x}+\frac{\left(\tilde{v}_{i, j+\frac{1}{2}}-\tilde{v}_{i, j-\frac{1}{2}}\right)}{\delta y} .
\end{aligned}
$$

\section{- Equações para $\psi$ nas Superfícies Livres:}

Para as superfícies livres, quatro exemplos de configurações serão apresentados como ilustração das equações discretizadas para os métodos Projeção-1 e Projeção-2 com FI. Nos outros casos, a forma de se obter as equações são análogas.

\section{- Caso 1:}


Nesse caso, de acordo com a figura 6.2, pode-se obter a equação para $\psi$ discretizada para o método Projeção-1, baseado na equação (5.42), como

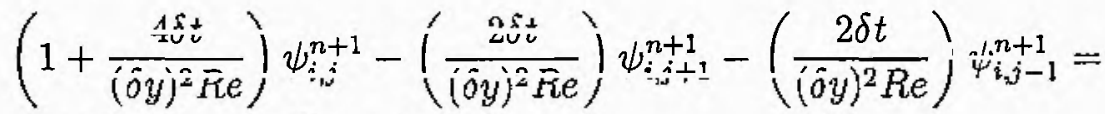

$$
\begin{aligned}
& -\frac{2 \delta t}{R e}\left(\frac{v_{i, j+\frac{1}{2}}-\tilde{v}_{i, j-\frac{1}{2}}}{\delta y}\right)
\end{aligned}
$$

e para o método Projeção-2, baseado na equação (5.68), tem-se

$$
\begin{aligned}
& \left(1+\frac{4 \delta t}{(\delta y)^{2} R e}\right) \psi_{i, j}^{n+1}-\left(\frac{2 \delta t}{(\delta y)^{2} R e}\right) \psi_{i, j+1}^{n+1}-\left(\frac{2 \delta t}{(\delta y)^{2} R e}\right) \psi_{i, j-1}^{n+1}= \\
& -\frac{2 \delta t}{R e}\left(\frac{\tilde{v}_{i, j+\frac{1}{2}}-\tilde{v}_{i, j-\frac{1}{2}}}{\delta y}\right)-\delta t \tilde{p}_{i, j} .
\end{aligned}
$$

Note que

$$
\left.\left(\frac{\partial \tilde{v}}{\partial y}\right)\right|_{i, j}=\frac{\tilde{v}_{i, j+\frac{1}{2}}-\vec{v}_{i, j-\frac{1}{2}}}{\delta y}
$$

pois a malha utilizada é deslocada, e portanto, as velocidades são dadas em numeração fracionária.

\begin{tabular}{|l|l|}
\hline $\begin{array}{c}\mathrm{S} / \mathrm{F} / \mathrm{B} / \mathrm{O} / \mathrm{I} \\
i, j+1\end{array}$ & $\mathrm{E}$ \\
\hline $\mathrm{S}$ & $\mathrm{E}$ \\
$i_{i, j}$ & \\
\hline $\mathrm{S} / \mathrm{F} / \mathrm{B} / \mathrm{O} / \mathrm{I}$ & $\mathrm{E}$ \\
$\bullet$ & \\
$i, j-1$ & \\
\hline
\end{tabular}

Figura 6.2: Configuração de células discretizadas - Caso 1.

A configuração da figura 6.2 , mostra que a célula computacional superior $(i, j+1)$, e a célula inferior $(i, j-1)$, em relação à célula $(i, j)$, ambas, podem ser do tipo S, F, B, O ou I (ver classificação das células na subseção 5.2.1). Nesse caso, conforme o tipo de célula, deve-se aplicar as corretas condições 
de contorno corretas, como apresentado nas subseções 5.3.1 e 5.4.1. Essa construção é análoga para os demais casos.

- Caso 2:

A configuração desse caso pode ser observado na figura 6.3 .

\begin{tabular}{|c|c|c|}
\hline $\mathrm{E}$ & $E$ & $\mathrm{E}$ \\
\hline $\mathrm{S} / \mathrm{F} / \mathrm{B} / \mathrm{O} / \mathrm{I}$ & $S$ & $\mathrm{~S} / \mathrm{F} / \mathrm{B} / \mathrm{O} / \mathrm{I}$ \\
\hline - & - & $\bullet$ \\
\hline$i-1, j$ & $i, j$ & $i+1, j$ \\
\hline
\end{tabular}

Figura 6.3: Configuração de células discretizadas - Caso 2.

Baseado na equação (5.47), para o método de Projeção-1, a equação discreta para o cálculo de $\psi$ é dada

$$
\begin{aligned}
& \left(1+\frac{4 \delta t}{(\delta x)^{2} R e}\right) \dot{\psi}_{i, j}^{-1}-\left(\frac{2 \delta t}{(\delta x)^{2} R e}\right) \dot{\psi}_{i+1, j}^{+1}\left(\frac{2 \delta t}{(\delta x)^{2} R e}\right) \psi_{i-1, j}^{n+1}= \\
& -\frac{2 \delta t}{R e}\left(\frac{\bar{u}_{i+\frac{1}{2}, j}-\tilde{u}_{i-\frac{1}{2}, j}}{\delta x}\right),
\end{aligned}
$$

e para o método de Projeção-2, baseado na equação (5.69), tem-se

$$
\begin{aligned}
& \left(1+\frac{4 \delta t}{(\delta x)^{2} R e}\right) \psi_{i, j}^{n+1}-\left(\frac{2 \delta t}{(\delta x)^{2} R e}\right) \psi_{i+1, j}^{n+1}-\left(\frac{2 \delta t}{(\delta x)^{2} R e}\right) \dot{\psi}_{i-1, j}^{n+1}= \\
& -\frac{2 \delta t}{R e}\left(\frac{\tilde{u}_{i+\frac{1}{2}, j}-\bar{u}_{i-\frac{1}{2} . .5}}{\delta x}\right)-\delta t \bar{p}_{i, j} .
\end{aligned}
$$

- Caso 3:

Os casos em que uma célula $\mathrm{S}$ é adjacente a duas células $\mathrm{E}$, devem ser tratados de forma especial na discretização. Deve-se utilizar a aproximação conforme a situação ou o caso em que a célula $S$ se encontra. Como exemplo, o Caso 3 (ver figura 6.4) será apresentado, e os outros casos, que também têm duas células adjacentes, devem seguir a mesma construção para se obter as equações discretizadas para $\psi$. 


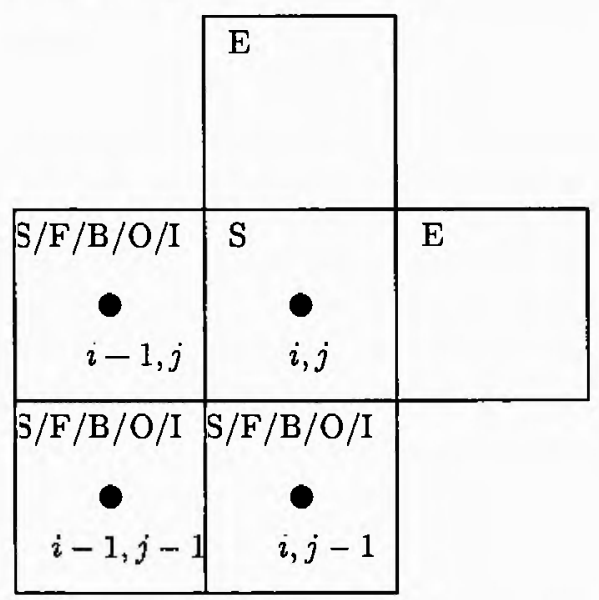

Figura 6.4: Configuração de células discretizadas - Caso 3.

Para o método de Projeção-1, baseado na equação (5.50), a forma discreta dessa equação é

$$
\begin{aligned}
& \left(1+\frac{2 \delta t}{(\delta x \delta y) R e}\right)^{\psi_{i, j}^{n+1}}-\left(\frac{2 \delta t}{(\delta x \delta y) R e}\right) \psi_{i, j-1}^{n+1}-\left(\frac{2 \delta t}{(\delta x \delta y) R e}\right) \psi_{i-1, j}^{n+1}+ \\
& \left(\frac{2 \delta t}{(\delta x \delta y) R e}\right)_{\substack{l, n+1 \\
r-1, j-1}}^{l, n+}=\left(\frac{\delta t}{R e}\right)\left(\frac{\tilde{u}_{i+\frac{1}{2}, j}+\tilde{u}_{i-\frac{1}{2}, j}-\tilde{u}_{i+\frac{1}{2}, j-1}-\tilde{u}_{i-\frac{1}{2}, i-1}}{2 \delta y}\right. \\
& \left.+\frac{\ddot{v}_{i, j+\frac{1}{2}}+\tilde{v}_{i, j-\frac{1}{2}}-\tilde{v}_{i-1, j+\frac{1}{2}}-\tilde{v}_{i-1, j-\frac{1}{2}}}{2 \delta x}\right) \text {. }
\end{aligned}
$$

Note que

$$
\begin{aligned}
& \left.\left(\frac{\partial^{2} \psi}{\partial x \partial y}\right)\right|_{i, j}=\frac{\partial}{\partial y}\left(\frac{\psi_{i, j}-\psi_{i-1, j}}{\delta x}\right)= \\
& \frac{1}{\delta x \delta y}\left(\psi_{i, j}-\psi_{i, j-1}\right)-\frac{1}{\delta x \delta y}\left(\psi_{i-1, j}-\psi_{i-1, y-1}\right)
\end{aligned}
$$

e devido à malha deslocada,

$$
\begin{aligned}
& \left.\left(\frac{\partial \tilde{u}}{\partial y}\right)\right|_{i, j}=\frac{\tilde{u}_{i+\frac{1}{2}, j}+\tilde{u}_{i-\frac{1}{2}, j}-\tilde{\dot{\omega}}_{i+\frac{1}{2}, j-1}-\tilde{u}_{i-\frac{1}{2}, j-1}}{2 \delta y} \\
& \left.\left(\frac{\partial \tilde{v}}{\partial x}\right)\right|_{i, j}=\frac{\tilde{v}_{i, j+\frac{1}{2}}+\tilde{v}_{i, j-\frac{1}{2}}-\tilde{\hat{v}}_{i-1, j+\frac{1}{2}}-\tilde{v}_{i-1, j-\frac{1}{2}}}{2 \delta x} .
\end{aligned}
$$


Baseado na equação (5.70), a equação discretizada para o método de Projeção2 nesse caso é

$$
\begin{aligned}
& \left(1+\frac{2 \delta t}{(\delta x \delta y) R e}\right) \dot{\psi}_{i, j}^{n+1}-\left(\frac{2 \delta t}{(\delta x \delta y) R e}\right) \psi_{i, j-1}^{n+1}-\left(\frac{2 \delta t}{(\delta x \delta y) R e}\right) \psi_{i-1, j}^{n+1}+ \\
& \left(\frac{2 \delta t}{(\delta x \delta y) R e}\right)^{i, \psi_{i-1, j-1}}=\left(\frac{\delta t}{R e}\right)\left(\frac{\bar{u}_{i+\frac{1}{2}, j}+\bar{u}_{i-\frac{1}{2}, j}-\tilde{u}_{i+\frac{1}{2}, j-1}-\tilde{u}_{i-\frac{1}{2}, j-1}}{2 \delta y}\right. \\
& \left.+\frac{\tilde{v}_{i, j: \frac{1}{2}}+\tilde{v}_{i, j-\frac{1}{2}}-\tilde{v}_{i-1, j: \frac{1}{2}}-\tilde{v}_{i-1, j-\frac{1}{2}}}{2 \delta x}\right)-\delta t \tilde{p}_{i, j} .
\end{aligned}
$$

- Caso 4:

Para esse caso, a configuração pode ser observada na figura 6.5 , e baseado na equação (5.53), tem-se a equação discretizada para o método de Projeção-1 como

$$
\begin{aligned}
& \left(1+\frac{2 \delta t}{(\delta x \delta y) R e}\right) \psi_{i, j}^{n+1}-\left(\frac{2 \delta_{t}}{(\delta x \delta y) R e}\right) \psi_{i, j+1}^{n+1}+\left(\frac{2 \delta t}{(\delta x \delta y) R e}\right) \psi_{i-1, j+1}^{n+1}- \\
& \left(\frac{2 \delta t}{(\delta x \delta y) R e}\right){ }_{i-1, j}^{n+1}=-\left(\frac{\delta t}{R e}\right)\left(\frac{\tilde{u}_{i+\frac{1}{2}, j+1}+\tilde{u}_{i-\frac{1}{2}, j+1}-\tilde{u}_{i+\frac{1}{2}, j}-\bar{u}_{i-\frac{1}{2}, j}}{2 \delta y}\right. \\
& \left.+\frac{\tilde{v}_{i, j+\frac{1}{2}}+\tilde{v}_{i, j-\frac{1}{2}}-v_{i-1, j+\frac{1}{2}}-\tilde{v}_{i-1, j-\frac{1}{2}}}{2 \delta x}\right),
\end{aligned}
$$

e para o método de Projeção-2, a equação (5.71) é discretizada na forma

$$
\begin{aligned}
& \left(1+\frac{2 \delta t}{(\delta x \delta y) R e}\right) \psi_{i, j}^{n+1}-\left(\frac{2 \delta t}{(\delta x \delta y) R e}\right) \psi_{i, j+1}^{n+1}+\left(\frac{2 \delta t}{(\delta x \delta y) R e}\right) \psi_{i-1, j+1}^{n+1}- \\
& \left(\frac{2 \delta t}{(\delta x \delta y) R e}\right) \psi_{i-1, j}^{n+1}=-\left(\frac{\delta t}{R e}\right)\left(\frac{\tilde{u}_{i+\frac{1}{2}, j+1}+\tilde{u}_{i-\frac{1}{2}, j+1}-\tilde{\tilde{\omega}}_{i+\frac{i}{2}, j}-\tilde{u}_{i-\frac{1}{2}, j}}{2 \delta y}\right. \\
& \left.+\frac{\tilde{v}_{i, j+\frac{1}{2}}+\tilde{v}_{i, j-\frac{1}{2}}-\tilde{v}_{i-1, j+\frac{1}{2}}-\tilde{v}_{i-1, j-\frac{1}{2}}}{2 \delta x}\right)-\delta t \tilde{p}_{i, j} .
\end{aligned}
$$




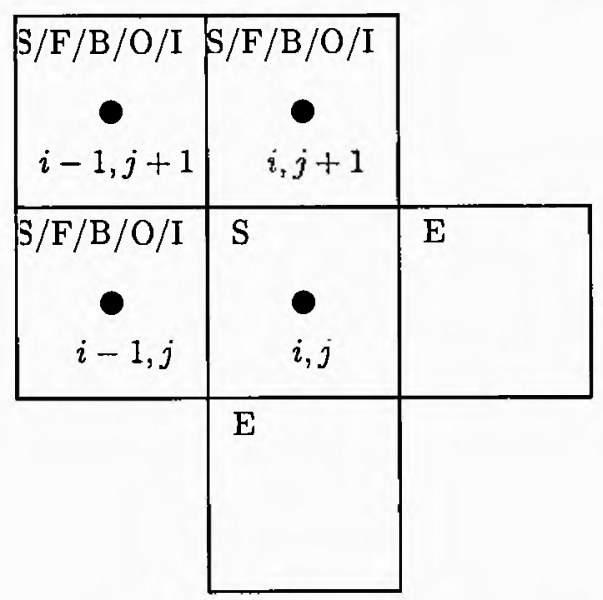

Figura 6.5: Configuração de células discretizadas - Caso 4.

\subsubsection{Técnicas de Armazenagem}

Como discutido na seção 5.5, a discretização implícita das condições de contorno produz uma matriz A esparsa e não simétrica para as equações no cálculo de $\psi$. Desta forma, foi necessário o estudo de técnicas de armazenagem compacta para essa matriz. Existem várias técnicas de armazenamento, como as descritas por (Knuth, 1968), (Kincaid et al., 1982) e (Bentley, 1986). Adota-se, no presente trabalho, a técnica de indexação por linhas descrita por (Bentley, 1986).

Para representar a matriz $\mathbf{A}$ de dimensão $n \times n$, a técnica de indexação por linhas necessita de dois vetores unidimensionais, sa e $i j a$. A seguir, um resumo do esquema para aplicação desse técnica é apresentado.

1. As primeiras $n$ posições do vetor sa armazenam os elementos da diagonal principal da matriz A;

2. A posição $n+1$ do vetor sa é um valor qualquer, aqui denotado $\mathrm{x}$;

3. As posições $>n+1$ do vetor sa armazena os demais elementos da matriz, percorrendo-a por linha;

4. As primeiras $n$ posições do vetor ija armazenam os índices do vetor sa nos quais estão armazenados o primeiro elemento fora da diagonal principal de cada linha;

5. A primeira posição do vetor ija é sempre igual a $n+2$; 
6. A posição $n+1$ do vetor $i j a$ é o último índice do vetor sa adicionando 1 ;

7. As posições $>n+1$ do vetor $i j a$ armazenam o número da coluna em que os elementos fora da diagonal pertencem, percorrendo a matriz por linha.

Como exemplo da aplicação dessa técnica, considere a matriz

$$
A=\left(\begin{array}{lllll}
1 & 0 & 2 & 0 & 0 \\
0 & 3 & 4 & 0 & 0 \\
0 & 5 & 6 & 7 & 0 \\
0 & 0 & 0 & 8 & 9 \\
0 & 0 & 0 & 1 & 2
\end{array}\right)
$$

Utilizando a técnica de indexação por linhas para $\mathbf{A}$, constroem-se os vetores sa e $i j a$ da seguinte forma

\begin{tabular}{|c||c|c|c|c|c|c|c|c|c|c|c|c|}
\hline índice $k$ & 1 & 2 & 3 & 4 & 5 & 6 & 7 & 8 & 9 & 10 & 11 & 12 \\
\hline i ja $[k]$ & 7 & 8 & 9 & 11 & 12 & 13 & 3 & 3 & 2 & 4 & 5 & 4 \\
\hline $\mathbf{s a}[k]$ & 1 & 3 & 6 & 8 & 2 & $x$ & 2 & 4 & 5 & 7 & 9 & 1 \\
\hline
\end{tabular}

Desta forma, pode-se armazenar uma matriz esparsa $\mathbf{A}$ em apenas dois vetores.

\subsubsection{Discretização das Equações de Atualização}

Nas seções 5.3 e 5.4, os passos finais dos algoritmos do método de Projeção-1 e Projeção-2 utilizando as Formulações Implícitas, fazem a atualização do campo final de velocidade e de pressão. Essa atualização, no caso do campo de velocidade final, é feita pela equação (5.18) para o método de Projeção-1 e para Projeção-2. Finalmente, o campo de pressão é atualizado por (5.41) no método de Projeção-1, e pela equação (5.19), no método de Projeção-2. Desta forma, essas equações são discretizadas como:

- Equação para Atualização da Velocidade:

- Método de Projeção-1 e Método de Projeção-2:

A equação (5.18), na direção $x$ é discretizada como

$$
u_{i+\frac{1}{2}, j}^{n+1}=\tilde{u}_{i+\frac{1}{2}, j}-\left(\frac{\psi_{i+1, j}^{n+1}-\psi_{i, j}^{n+1}}{\delta x}\right),
$$


e na direção $y$

$$
v_{i, j+\frac{1}{2}}^{n+1}=\tilde{v}_{i, j+\frac{1}{2}}-\left(\frac{\psi_{i, j+1}^{n+\cdot 1}-\psi_{i, j}^{n+\dot{r} \cdot 1}}{\delta y}\right) .
$$

- Equação para Atualização da Pressão:

- Método de Projeção-1:

A equação (5.41) é discretizada como

$$
p_{i, j}^{n+1}=\frac{\psi_{i, j}^{n+1}}{\delta t_{t}}
$$

- Método de Projeção-2:

A equação (5.19) é discretizada como

$$
p_{i, j}^{n+1}=\tilde{p}_{i, j} \div \frac{\psi_{i, j}^{n+1}}{\delta t}
$$

\subsection{Ambiente de Simulação FreeFlow2D}

Neste trabalho, utilizou-se o ambiente de simulação FreeFlow2D, para se implementar as rotinas e funções para as Formulações Implícitas na metodologia GENSMAC. A estrutura de dados utilizada no FreeFlow2D foi baseada no ambiente FreeFlow3D (Castelo et al., 1996), e bem discutida em (Oliveira, 1999).

O sistema FreeFlow2D é um sistema computacional para modelagem, simulação e visualização de escoamentos de fluidos que possibilita a análise e a observação do comportamento dinâmico de fluidos incompressíveis. Desta forma, esse sistema dividi-se em módulos, e a comunicação é feita por arquivos. Detalhes sobre os módulos e a comunicação dos módulos podem ser encontrados em (Castelo et al., 1996), (Oliveira, 1999) e (Oliveira, 2000). A implementação dos módulos que compõem o sistema FreeFlow2D foi feita utilizando a linguagem C. A seguir apresenta-se um resumo sobre esses módulos.

\subsubsection{Modelador}

O modelador bidimensional é um editor gráfico interativo que permite a definição dos objetos bidimensionais do escoamento de fluidos, que inclui os dados que configuram o escoamento. Esses dados são: domínio, número de células, dimensão das células, 
tempo inicial e final, ciclo inicial e final, intervalo de tempo para impressão e gravação automática, escalas de comprimento e velocidade, força de gravidade, viscosidade, incremento de tempo inicial, tolerância para resolução dos sistemas lineares, fatores de controle no tempo, tipo de fluido para o escoamento e opções do método de resolução: técnicas explícitas ou técnicas implícitas (as técnicas implícitas foram implementas neste trabalho). Como exemplo da interface principal do modelador com as propriedades e dados do escoamento, pode-se ver a figura 6.6.

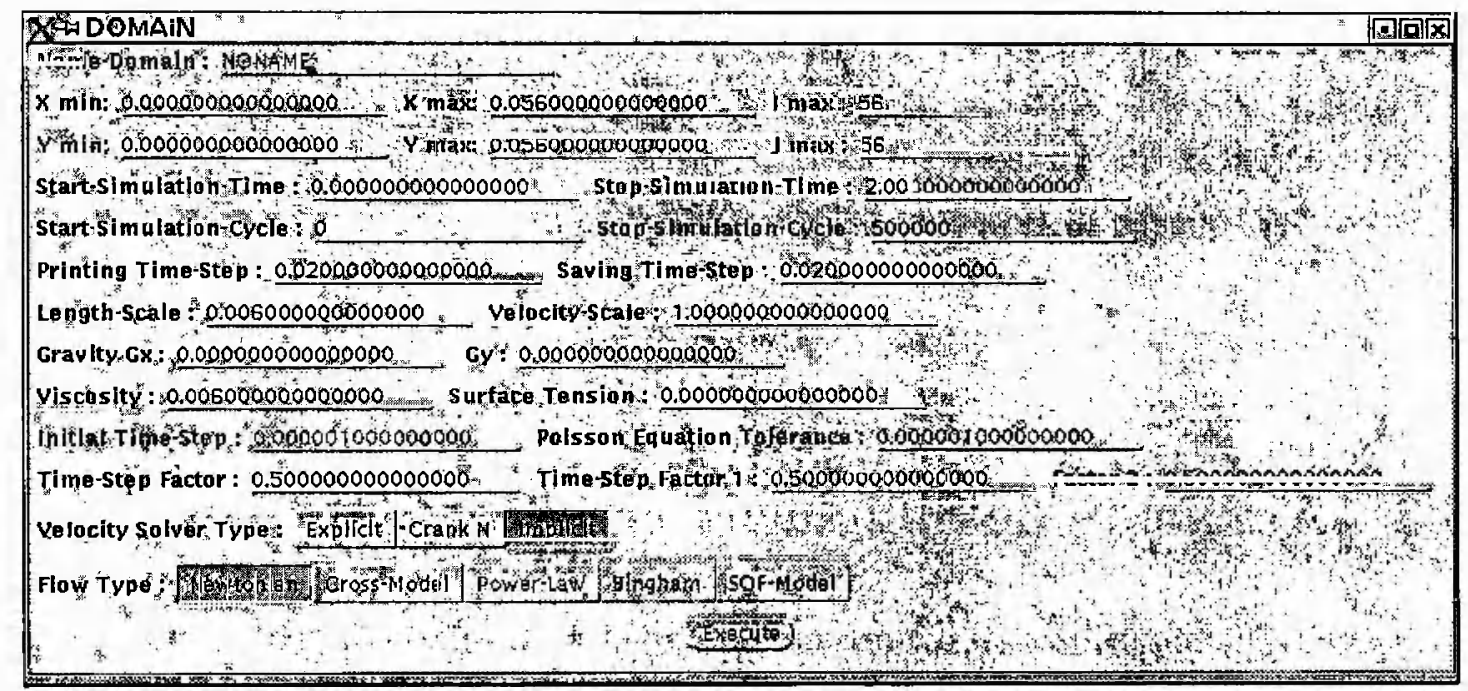

Figura 6.6: Interface gráfica do modelador.

Através da interface de entradas de dados, o usuário pode modificar os campos desejados e salvar a configuração em um arquivo.

\subsubsection{Simulador}

O simulador consiste em um conjunto de programas baseado na metodologia GENSMAC, cuja finalidade é resolver problemas de escoamentos transientes de fluidos incompressíveis e com superfícies livres. Nesse módulo, que não tem interface gráfica, as equações de Navier-Stokes e a equação da continuidade são resolvidas passo a passo como foi discutido no capítulo 5 pelo método de diferenças finitas. O simulador rèpresenta o fluido por partículas apenas na fronteira. Detalhes sobre a movimentação das partículas e a atualização das partículas marcadoras podem ser encontrados em (Oliveira, 1999). No presente trabalho, utilizou-se o módulo simulador do ambiente 
FreeFlow2D, para implementar as modificações na metodologia GENSMAC, incluindo os métodos de Projeção-1 e Projeção-2 com as Formulações Implícitas, as modificações no tratamento das condições de contorno nas superfícies livres e o método GBCP para os sistemas lineares.

\subsubsection{Visualizador}

Esse módulo é responsável pela visualização gráfica dos resultados da simulação de escoamentos de fluidos. O visualizador possui uma barra de menus com opções de manipulação de arquivos, escolha de tipos de variáveis para visualização, modificação de configurações, animação e ajuda. Escolhida uma propriedade, as superfícies dos fluidos são coloridas de acordo com o valor assumido por ela em cada ponto, e uma faixa de cores, relaciona o valor das propriedades para facilitar o entendimento da figura. Essa faixa também informa a variação total da propriedade visualizada. O visualizador possui uma interface gráfica de fácil execução como mostra a figura 6.7.

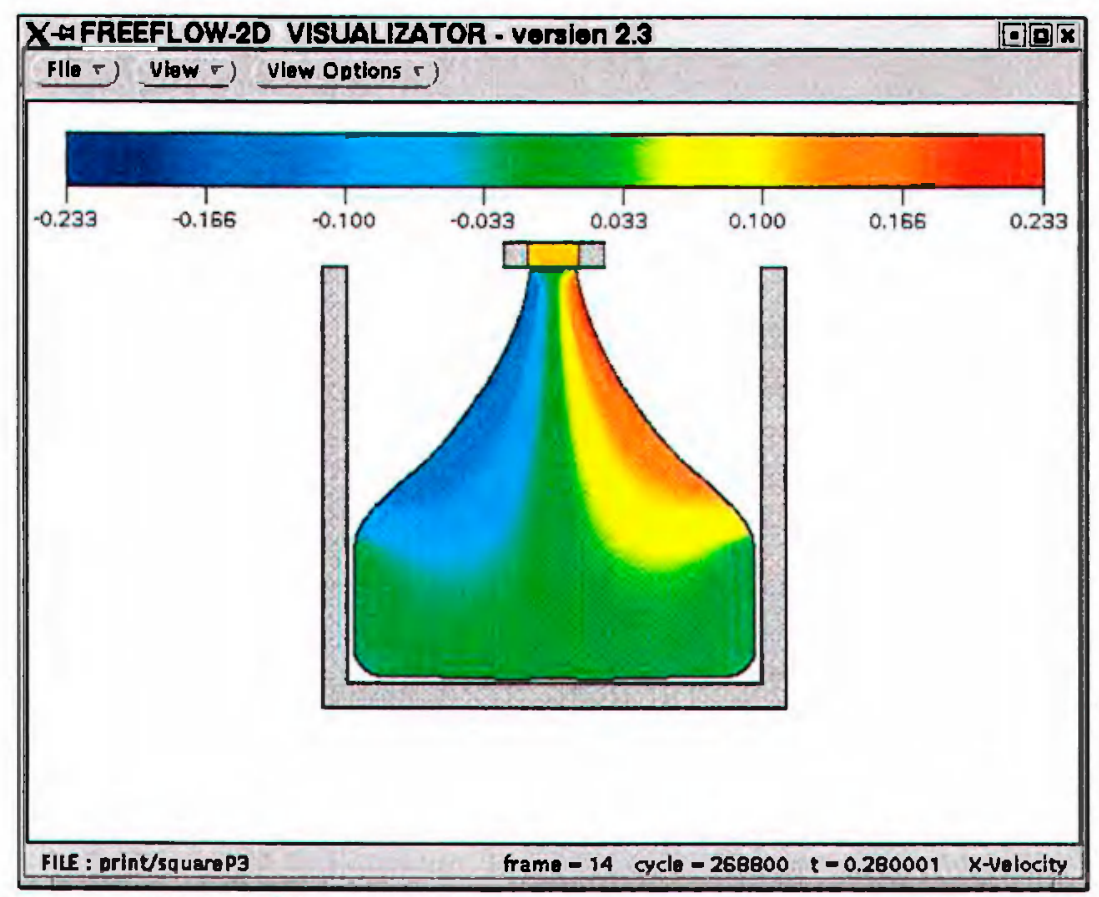

Figura 6.7: Interface gráfica do visualizador. 


\subsubsection{Re-inicializador}

O módulo re-inicializador é responsável em reiniciar a simulação do ponto em que parou ou em realizar modificações em algum campo para novas simulações. No caso de interrupção acidental pode-se utilizar o re-inicializador, que tem como dados de entrada o ciclo final, tempo final, intervalo de tempo para impressão e gravação, tolerância para o método que resolve os sistemas lineares envolvidos na simulação e os fatores controladores do tempo. A interface do re-inicializador pode ser observada na figura 6.8 .

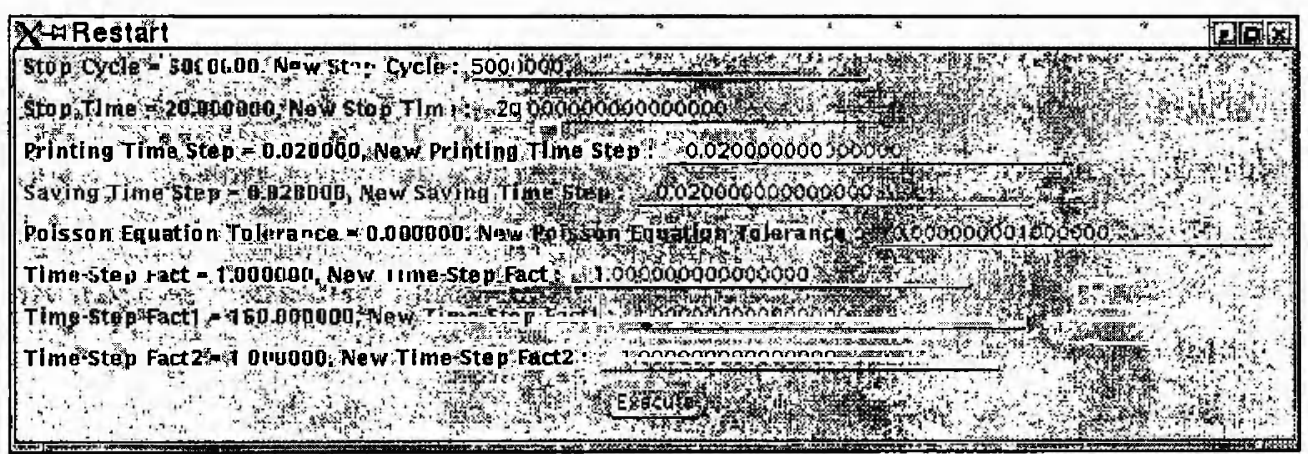

Figura 6.8: Interface gráfica do re-inicializador. 


\section{Resultados Numéricos}

\subsection{Introdução}

Neste capítulo, os métodos Projeção-1 e Projeção-2 utilizando Formulações Implícitas, analisados no capítulo 5, são agora aplicados na simulação de escoamentos de fluidos newtonianos incompressíveis para problemas bidimensionais na presença de contornos rígidos e/ou livres. Em geral, os números de Reynolds envolvidos nesses problemas são baixos ocasionando dificuldades na determinação das soluções numéricas quando aplica-se o método explícito.

Essa dificuldade ocorre, como apresentado anteriormente, devido às restrições de estabilidade em $\delta t$ dos métodos explícitos. Tais restrições, podem ser reduzidas utilizando formulações implícitas na metodologia GENSMAC. Porém, um cuidado na aplicação desses métodos implícitos é na escolha do passo temporal, para que não interfira na precisão dos resultados. Neste capítulo, apresentam-se os resultados de simulações numéricas obtidas com as formulações implícitas para comparações com o método explícito original. As comparações, têm como objetivo principal, mostrar a eficiência dos métodos Projeção-1 e Projeção-2 utilizando as formulações IR, CN e AB/CN, em relação ao método explícito, nos problemas de escoamentos com $R e<<1$. Tal eficiência, é clara nos resultados apresentados para o passo temporal, o número de iterações, o tempo de processamento e outras propriedades importantes para as comparações. Além 
das comparações. desses métodos, os resultados numéricos mostraram a diferença entre a formulação IR e CN, para diferentes problemas e simulações.

Em particular, os métodos numéricos foram aplicados aos seguintes problemas: Escoamento em um canal, para validar as técnicas numéricas; Jato incidindo sobre uma superfície rígida, para verificar a eficiência dos métodos em problemas com superfícies livres e preenchimento de uma caixa, para verificar a fidelidade dos resultados numéricos em comparação com experimentos. Para facilitar a notação em figuras e tabelas, os métodos Projeção-1 e Projeção-2, serão denotados por P1 e P2, e as formulações implícitas continuam sendo denotadas como anteriormente, IR, CN e AB/CN. O ambiente de simulação é o FreeFlow2D, descrito no capítulo 6. Todas as simulações numéricas neste trabalho foram realizadas em microcomputadores

- Dual Athlon XP MP 2200, 1.0 Gb de memória RAM, 80.0 Gb de disco rígido e sistema operacional Linux Slackware 9.0, encontrados no Laboratório de Computação de Alto Desempenho (LCAD) do ICMC-USP.

\subsection{Simulação do Escoamento em um Canal}

Uma importante etapa para a interpretação dos resultados numéricos é o estudo para a validação do código utilizado. A etapa de validação é importante pois fornece evidências de que o método numérico implementado é representativo de fenómenos físicos. Para o estudo da validação dos resultados numéricos fornecidos pelos métodos de Projeção-1 e Projeção-2 utilizando Formulações Implícitas, considera-se o escoamento de um fluido entre duas placas paralelas, conhecido como escoamento de HagenPoiseuille (ver figura 7.1).

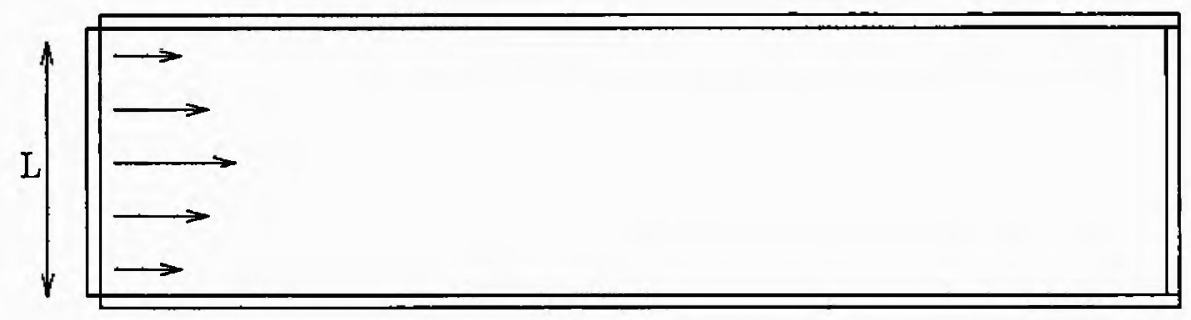

Figura 7.1: Domínio para um escoamento de Hagen-Poiseuille. O escoamento é da esquerda para a direita e o comprimento do canal é $5 L$. 
O escoamento de Hagen-Poiseuille, estudado por Hagen em 1839 e por Poiseuille em 1840, é muito importante pois ele é um dos poucos problemas para as equações de Navier-Stokes para o qual uma solução analítica existe, possibilitando a validação do código.

A solução analítica para este problema será brevemente discutida, podendo ser encontrada em (Batchelor, 1970). Em resumo, de acordo com (Batchelor, 1970), a velocidade $u$ na direção do escoamento e a uma distância $y$ da parede inferior do canal é dada pela expressão

$$
u(y)=-\frac{1}{2 \mu} \frac{\partial_{\tilde{\mu}}}{\partial \hat{y}}\left(y L-y^{2}\right)
$$

onde $\mu$ é o coeficiente de viscosidade. O gradiente de pressão é dado pela expressão

$$
\frac{\partial p}{\partial x}=-12 \frac{\mu Q}{L^{3}}
$$

e a vazão $Q$ é definida como

$$
Q=\int_{0}^{\vec{\nu}} u(y) d y
$$

Para obter a solução analítica (7.1) da velocidade em função do gradiente de pressão deve-se definir um perfil de velocidade. Desta forma defini-se $u$ como uma velocidade normal à fronteira de entrada de fluido e $U$ uma velocidade de referência. Quando

$$
u(y)=U\left(1-\frac{y^{2}}{L^{2}}\right)
$$

- perfil de velocidade é do tipo parabólico, e quando

$$
u(y)=U
$$

defini-se o perfil de velocidade do tipo reto.

A prescrição de um perfil de velocidade é fundamental, pois muitas vezes, não é possível conhecer a distribuição de velocidades através de uma fronteira de fluido, sendo conhecida apenas a vazão ou a descarga. Desta forma, a solução é prescrever um perfil de velocidade arbitrário, mas compatível com o problema tratado. Após determinar 0 perfil de velocidade, pode-se calcular a solução analítica para o escoamento de HagenPoiseuille. Para isto, é necessário fixar o valor de $L$ e da velocidade de referência $U$. 
A vazão $Q$ para o perfil parabólico é dada pela expressão

$$
Q=\int_{0}^{L} u(y) d y=\int_{0}^{L} U\left(1-\frac{y^{2}}{L^{2}}\right) d y
$$

e para o perfil reto,

$$
Q=\int_{J_{0}}^{\bar{\nu}} u(y) d y=\int_{J_{0}}^{\bar{\nu}} U d y
$$

Escolhendo $L=1$ e $U-1$, a vazão $Q$ é calculada diretamente, sendo possível então, determinar o valor do gradiente de pressão (7.2), ou seja

$$
\frac{\partial p}{\partial x}=-8 \mu, \text { para o perfil parabólico, }
$$

$\mathrm{e}$

$$
\frac{\partial p}{\partial x}=-12 \mu, \text { para o perfil reto }
$$

Portanto, substituindo a equação (7.8) em (7.1) obtêm-se a solução analítica para o perfil parabólico, na forma

$$
u(y)=-4 y(y-1)
$$

e no caso do perfil reto, ao substituir (7.9) em (7.1) tem-se

$$
u(y)=-6 y(y-1)
$$

Como exemplo para a validação dos métodos numéricos deste trabalho, considere duas placas paralelas separadas a uma distância $L=1$, formando um canal, que no inicio da simulação está completamente vazio e o fluido é injetado na entrada do canal à uma velocidade prescrita e com perfil do tipo parabólico. As condições para escoamento totalmente desenvolvido impostas na entrada de fluido são:

$$
u(y)=-4 y(y-L)=-4 y(y-1), \quad v=0
$$

$\mathrm{Na}$ figura 7.2 observa-se o desenvolvimento da superfície livre do fluido, para o escoamento de Hagen-Poiseuille. No tempo $t=0$ o canal está vazio e o fluido é injetado até atingir a saída do canal, e o estado estacionário se estabelece. Quando atỉnge-se o estado estacionário, os valores das velocidades ao longo do canal são os mesmos que os impostos na entrada de fluido, e desta forma, pode-se comparar os resultados 
a)

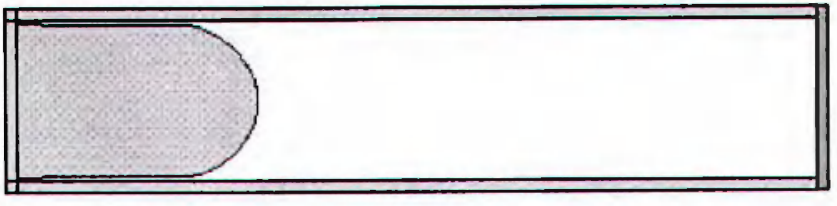

b)

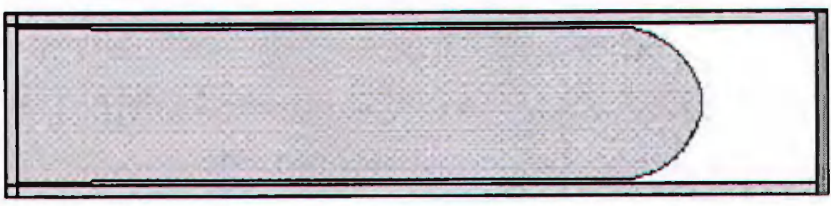

c)

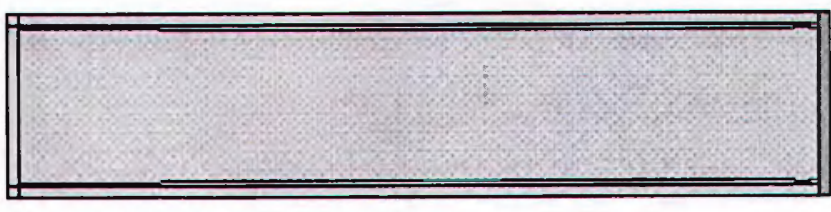

Figura 7.2: Simulação numérica do escoamento de Hagen-Poiseuille. Superfície do fluido nos tempos: a) $t=2.0 \mathrm{~s}, \mathbf{b}) t=6.0 \mathrm{~s} \mathrm{e} \mathrm{c)} t=12.0 \mathrm{~s}$.

numéricos com a solução analítica dada pela expressão (7.11). Após a descrição da solução analítica e o desenvolvimento do fluido dentro do canal, as comparações podem ser realizadas, a partir de um modelo para simulação. O modelo para esse escoamento é descrito a seguir:

- Dimensão do domínio: $5.6 \mathrm{~m} \times 1.6 \mathrm{~m}$ - (malha grossa); $5.3 \mathrm{~m} \times 1.3 \mathrm{~m}$ - (malha intermediária); $5.15 \mathrm{~m} \times 1.15 \mathrm{~m}$ - (malha fina);

- Diâmetro do injetor $(L): 1.0 \mathrm{~m}$;

- Velocidade de injeção $(U): 1.0 \mathrm{~ms}^{-1}$;

- Coeficiente de viscosidade cinemática $(\nu): 10.0 \mathrm{~m}^{2} \mathrm{~s}^{-\mathrm{i}}$;

- Parâmetros de escala: $L=1.0 \mathrm{~m}$ e $U=1.0 \mathrm{~ms}^{-1}$;

- Número de Reynolds $(R e=U L / \nu)$ : 0.1;

- Número de Froude $(F r=U / \sqrt{g} L): 0.319275$.

Os métodos Projeção-1 e Projeção-2 utilizando as FI foram aplicados para o escoamento de Hagen-Poiseuille sobre três malhas diferentes. A primeira malha, definida 
como grossa $(\mathrm{M} 1)$, tem tamanho de $50 \times 10$ células $(\delta x=\delta y=0.1 \mathrm{~m})$; a segunda, definida como intermediária (M2), $100 \times 20$ células $(00 x=\delta y=0.05 \mathrm{~m}$ ) e a terceira malha, definida como fina (M3), tem $200 \times 40$ células $(\delta x=\delta y=0.025 \mathrm{~m})$. A região considerada para a comparação dos resultados numéricos com a solução analítica, dada pela equação (7.1), para as três malhas, é no meio do canal, após o escoamento encontrar-se em estado estacionário. O escoamento atingiu o estado estacionário para a malha M1, em $t=10 \mathrm{~s}$, para a malha $\mathrm{M} 2 \mathrm{em} t=20 \mathrm{~s}$ e para a malha $\mathrm{M} 3, t=30 \mathrm{~s}$. Os valores de $\delta t$ nas figuras e tabelas são apresentados em segundos. No ambiente FreeFlow2D, em particular, no módulo do simulador, há uma rotina que calcula o tempo de processamento de cada simulação permitindo uma comparação sobre o tempo total do processo.

Como pode-se observar pelas figuras 7.3 e 7.4, os resultados numéricos são bastante semelhante à solução analítica, ou seja, os valores numéricos obtidos pelos métodos Projeção-1 e Projeção-2 utilizando as FI, sobre as três malhas, estão em boa concordância com a solução analítica.

Observa-se pela figura 7.3 que o método Projeção-1, independente da escolha da formulação implícita, mostrou uma pequena discrepância da solução analítica sobre a malha grossa, o que não aconteceu para o método Projeção-2, como pode-se ver na figura 7.4. Mas após o refino da malha, essa discrepância desaparece, mostrando a convergência do método Projeção-1.

Para melhor avaliar os resultados numéricos, e mostrar a convergência dos métodos numéricos apresentados neste trabalho, foi feito o cálculo do erro relativo $\left(E_{\text {rel }}\right)$ na norma $l_{2}$ entre as soluções numéricas e a solução analítica. O erro relativo é dado pela equação

$$
E_{\text {rel }}^{\prime}=\frac{\Sigma\left(u_{\text {analítica }}-u_{\text {numérica }}\right)^{2}}{\Sigma\left(u_{\text {analítica }}\right)^{2}}
$$

Uma síntese, do erro na norma $l_{2}$ e do passo temporal $\delta t$, para as malhas $\mathbf{M 1}$, M2 e M3, é apresentada na tabela 7.1. Esses resultados quantificam as observações consideradas nas figuras 7.3 e 7.4. Os resultados do método explícito, obtido pelo método GENSMAC, também são apresentados para comparação com os métodos que utilizam as FI. Observa-se pela tabela 7.1 que os erros decrescem, para os métodos Projeção-1 e Projeção-2 utilizando as FI, à medida que a malha é refinada, mostrando a convergência dos métodos numéricos. 
a)

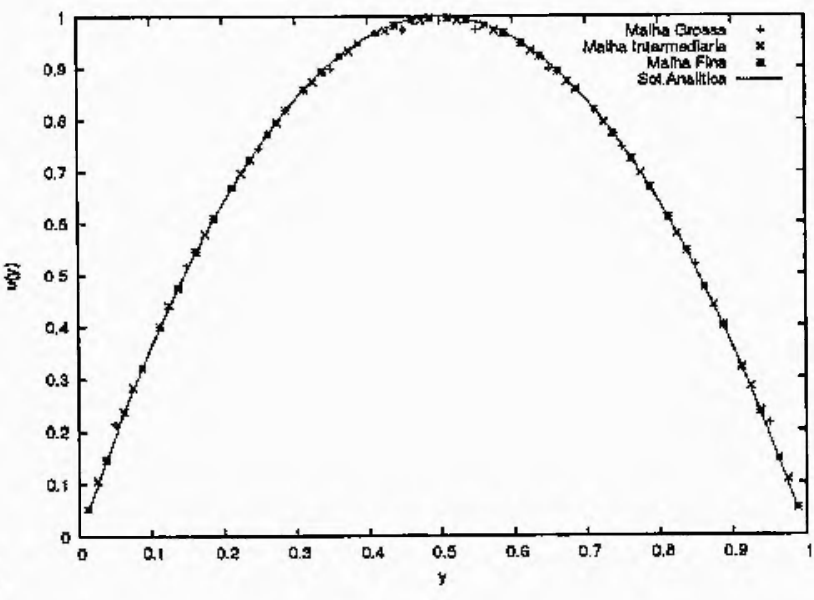

b)

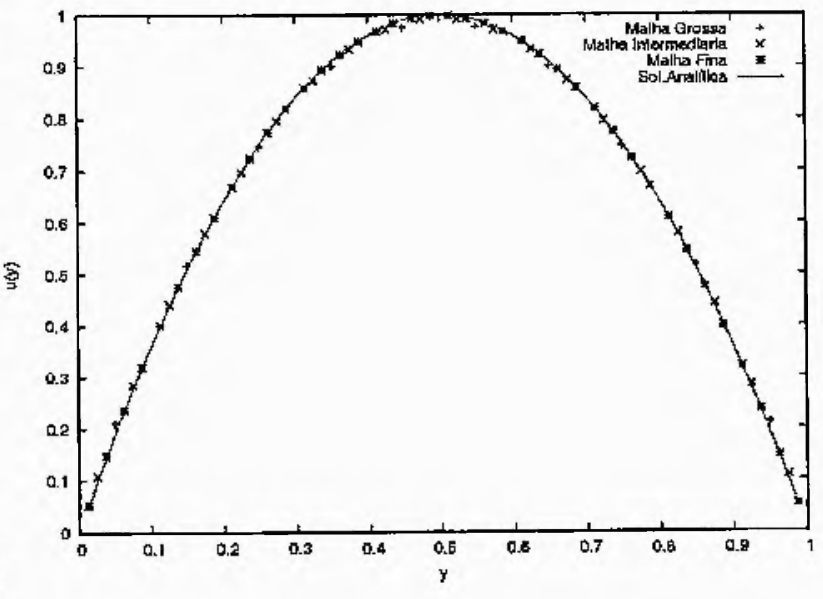

c)

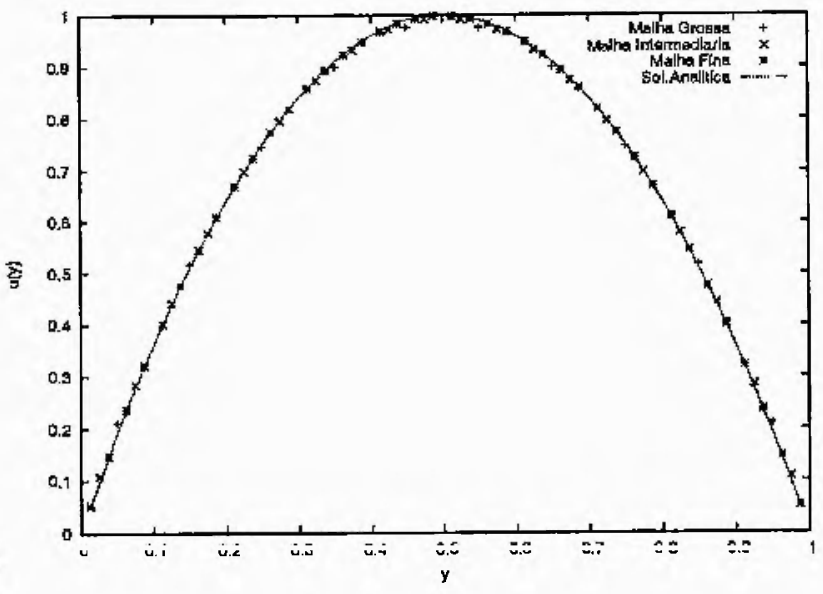

Figura 7.3: Comparação entre soluções numéricas obtida pelo método Projeção-1 utilizando FI e a solução analítica dada pela equação (7.11), sobre as três malhas, com $R e=0.1$. As figuras a)-c) correspondem ao método Projeção-1 utilizando as formulações IR, $\mathrm{CN}$ e $\mathrm{AB} / \mathrm{CN}$, respectivamente. 
a)

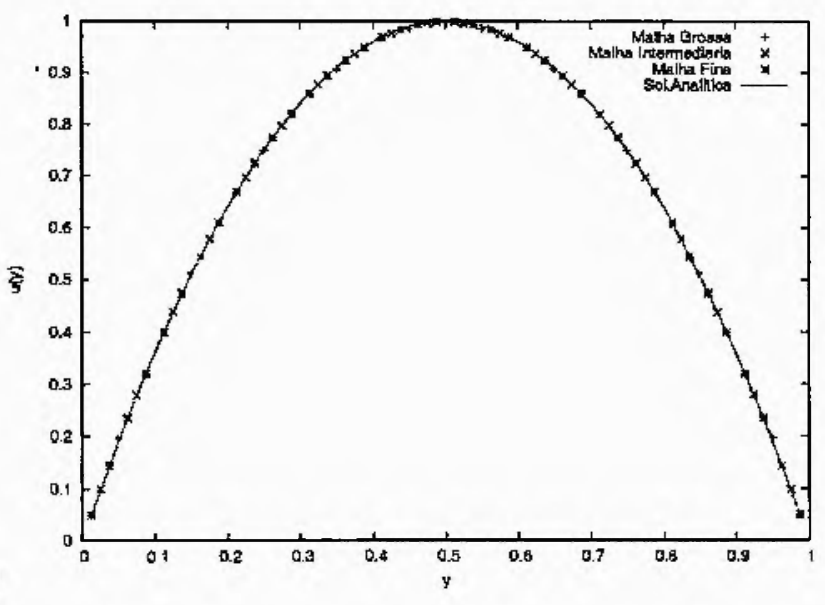

b)

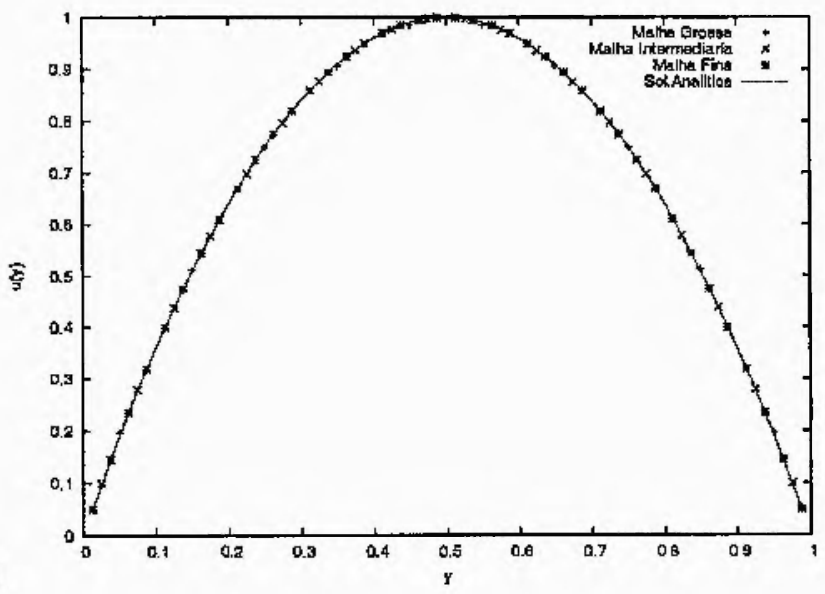

c)

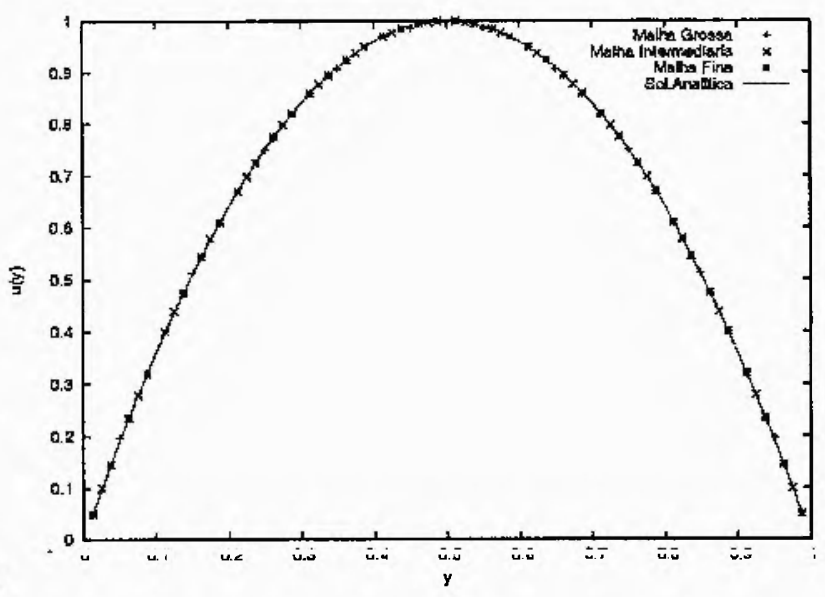

Figura 7.4: Comparação entre soluções numéricas obtida pelo método Projeção-2 utilizando FI e a solução analítica dada pela equação (7.11), sobre as três malhas, com $R e=0.1$. As figuras a)-c) correspondem ao método Projeçäo-2 utilizando as formulações IR, $\mathrm{CN}$ e $\mathrm{AB} / \mathrm{CN}$, respectivamente. 
Tabela 7.1: Resultados do escoamento de Hagen-Poiseuille para $R e=0.1$ nas malhas M1, M2 e M3. O valor do $\delta t$ ê dado em segundos após o escoamento atingir o estado estacionário.

\begin{tabular}{|c||c|c||c|c||c|c|}
\hline Método & $\delta t(\mathrm{~s})$ & $\mathrm{M} 1$ & $\delta t(\mathrm{~s})$ & $\mathrm{M} 2$ & $\delta t(\mathrm{~s})$ & $\mathrm{M} 3$ \\
\hline Explícito & $1.0 \times 10^{-4}$ & $2.5 \mathrm{E}-05$ & $2.5 \times 10^{-5}$ & $1.8 \mathrm{E}-06$ & $6.25 \times 10^{-6}$ & $1.3 \mathrm{E}-07$ \\
\hline P1-IR & $1.25 \times 10^{-3}$ & $7.1 \mathrm{E}-04$ & $2.5 \times 10^{-4}$ & $3.7 \mathrm{E}-05$ & $6.25 \times 10^{-5}$ & $3.2 \mathrm{E}-06$ \\
\hline P1-CN & $2.0 \times 10^{-3}$ & $5.6 \mathrm{E}-04$ & $5.0 \times 10^{-4}$ & $4.5 \mathrm{E}-05$ & $1.25 \times 10^{-4}$ & $3.2 \mathrm{E}-06$ \\
\hline P1-AB/CN & $2.0 \times 10^{-3}$ & $1.6 \mathrm{E}-04$ & $5.0 \times 10^{-4}$ & $2.5 \mathrm{E}-05$ & $1.25 \times 10^{-4}$ & $2.1 \mathrm{E}-06$ \\
\hline P2-IR & $1.0 \times 10^{-2}$ & $2.5 \mathrm{E}-05$ & $1.25 \times 10^{-2}$ & $1.8 \mathrm{E}-06$ & $6.25 \times 10^{-3}$ & $1.2 \mathrm{E}-07$ \\
\hline P2-CN & $2.0 \times 10^{-3}$ & $2.5 \mathrm{E}-05$ & $5.0 \times 10^{-4}$ & $1.8 \mathrm{E}-06$ & $5.0 \times 10^{-4}$ & $1.1 \mathrm{E}-07$ \\
\hline P2-AB/CN & $2.0 \times 10^{-3}$ & $2.5 \mathrm{E}-05$ & $5.0 \times 10^{-4}$ & $1.8 \mathrm{E}-06$ & $5.0 \times 10^{-4}$ & $1.1 \mathrm{E}-07$ \\
\hline
\end{tabular}

O pequeno desvio da solução analítica e da solução numérica do método Projeção-1, observado na figura 7.3 é confirmado pelos resultados mostrados na tabela 7.1. Conforme a tabela 7.1, observa-se que a solução numérica obtida com o método Projeção-1 utilizando as FI, em comparação com o método explícito e o método Projeção-2 com as FI, apresentou maiores erros. Esse fato mostra que o método Projeção-1, para determinados valores de $\delta t$, tem convergência temporal inferior ao método Projeção-2, independente da formulação implícita utilizada.

Com o objetivo de melhorar a convergência temporal do método Projeçäo-1, foi aplicado um esquema Adams de passo múltiplo, denominado neste trabalho como método $\mathrm{AB} / \mathrm{CN}$, apresentado na seção 5.3. Como pode-se ver pela tabela 7.1, esse esquema, de fato, apresentou um erro inferior, em relação às formulações IR e CN para o método Projeção-1 com o mesmo $\delta t$. Mesmo assim, os erros apresentados pelo método Projeção1 são significativamente maiores que os do método Projeção-2. De acordo com esses fatos, pode-se concluir, que eliminar o gradiente de pressão da equação de conservação de movimento (3.15), como foi visto na seção 5.3, o método Projeção-1 utilizando FI, tem como consequência uma precisão inferior. Por outro lado, o método de Projeção2 utilizando as formulações $\mathrm{IR}, \mathrm{CN}$ e $\mathrm{AB} / \mathrm{CN}$, mostrou resultados muito semelhantes entre suas formulações implícitas, com erros relativos pequenos e resultados em boa concordância. Em comparação com o método explícito. o método Projeção-2, apresentou erros relativos iguais nas malhas M1 e M2, e para a malha M3, os resultados obtidos foram melhores do que os obtidos pelo método explícito.

Um fato importante ocorreu quando aumentou-se o valor de $\delta t$ para o método Projeção-1, pois o erro relativo também aumentou, enquanto que na Projeção-2, apesar do crescimento do $\delta t$, o erro relativo foi praticamente constante. Esse fato pode ser ob- 
servado pelas figuras 7.5, 7.6 e 7.7, que apresentam o comportamento da convergência temporal dos métodos apresentados, para o escoamento de Hagen-Poiseuille sobre a malha intermediária com $R e=0.1$. As tabelas $7.2,7.3$ e 7.4 apresentam os resultados das figuras $7.5,7.6$ e 7.7 , respectivamente.

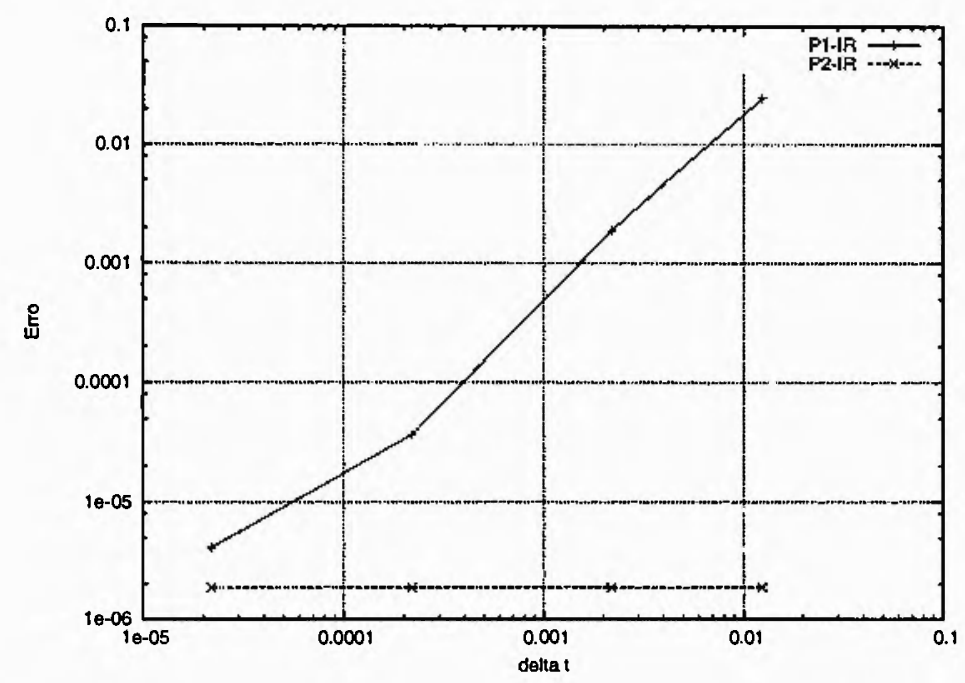

Figura 7.5: Erro relativo na norma $l_{2}$, para a velocidade $u$ e os métodos Projeção-1 e Projeção-2 utilizando a formulação IR sobre a malha M2.

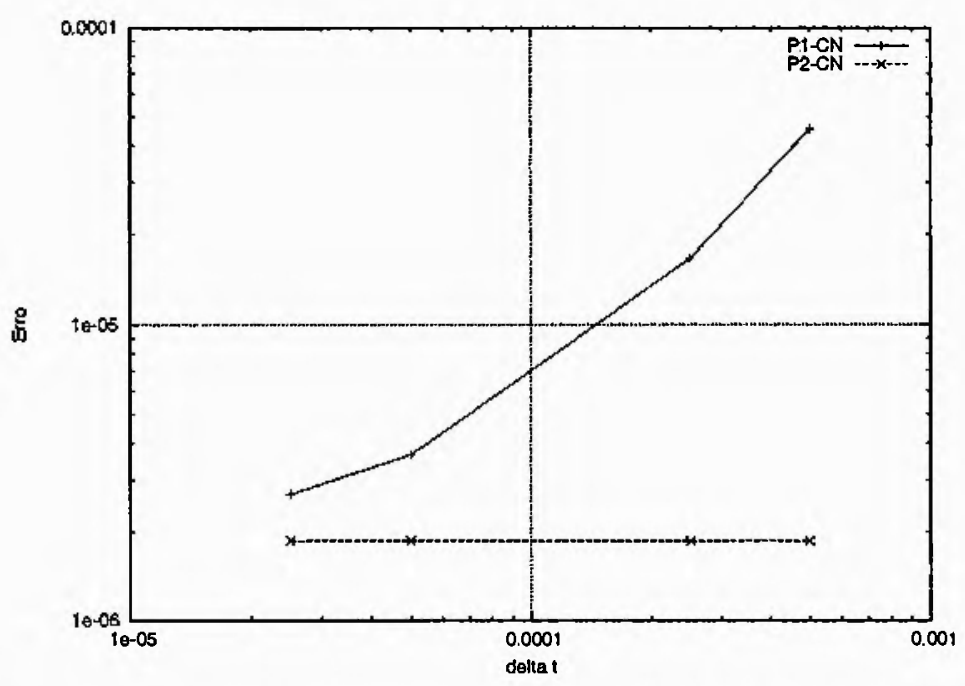

Figura 7.6: Erro relativo na norma $i_{2}$, para a velocidade $u$ e os métodos Projeção-1 e Projeção-2 utilizando a formulação CN sobre a malha M2. 


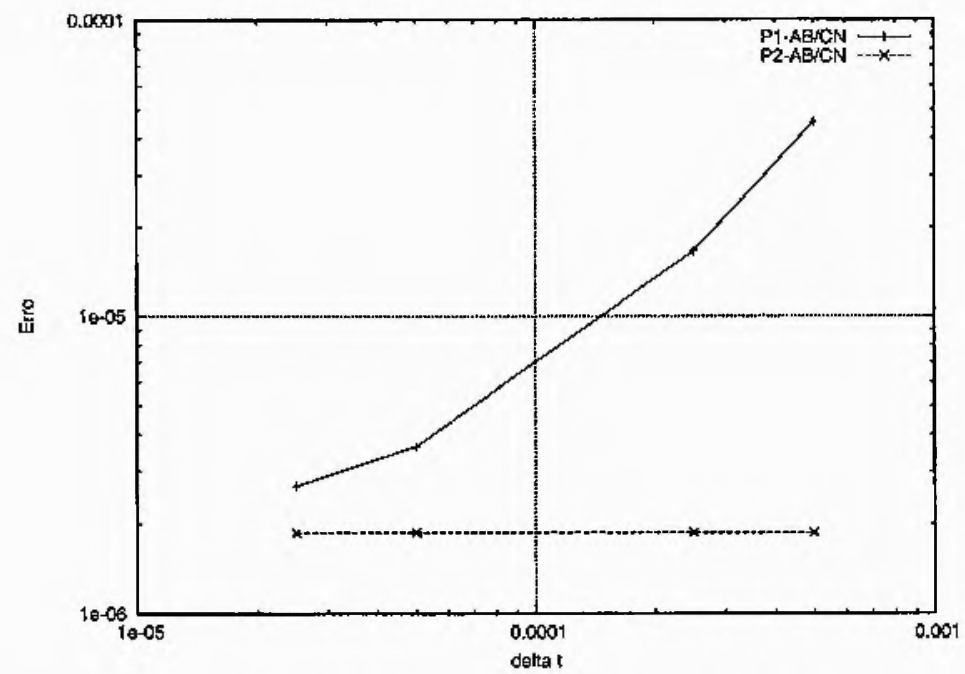

Figura 7.7: Erro relativo na norma $l_{2}$, para a velocidade $u$ e os métodos Projeção-1 e Projeção-2 utilizando a formulação $\mathrm{AB} / \mathrm{CN}$ sobre a malha M2.

Tabela 7.2: Comparação entre os métodos Projeção-1 e Projeção-2, ambos utilizando a formulação IR, para o erro relativo no escoamento de Hagen-Poiseuille sobre a malha M2 para $R e=0.1$.

\begin{tabular}{|c||c||c|}
\hline$\delta t(\mathrm{~s})$ & $P 1$-IR & P2-IR \\
\hline $2.1875 \times 10^{-5}$ & $4.1899 \times 10^{-6}$ & $1.8691 \times 10^{-6}$ \\
\hline $2.1875 \times 10^{-4}$ & $3.6879 \times 10^{-5}$ & $1.8691 \times 10^{-6}$ \\
\hline $2.1875 \times 10^{-3}$ & $1.8951 \times 10^{-3}$ & $1.8691 \times 10^{-6}$ \\
\hline $1.25 \times 10^{-2}$ & $2.5189 \times 10^{-2}$ & $1.8691 \times 10^{-6}$ \\
\hline
\end{tabular}

Tabela 7.3: Comparação entre os métodos Projeção-1 e Projeção-2, ambos utilizando a formulação CN, para o erro relativo no escoamento de Hagen-Poiseuille sobre a malha M2 para $R e=0.1$.

\begin{tabular}{|c||c||c|}
\hline$\delta t(\mathrm{~s})$ & $P 1-\mathrm{CN}$ & $P 2-\mathrm{CN}$ \\
\hline $2.5 \times 10^{-5}$ & $2.6875 \times 10^{-6}$ & $1.8691 \times 10^{-6}$ \\
\hline $5.0 \times 10^{-5}$ & $3.6525 \times 10^{-6}$ & $1.8691 \times 10^{-6}$ \\
\hline $2.5 \times 10^{-4}$ & $1.6575 \times 10^{-5}$ & $1.8691 \times 10^{-6}$ \\
\hline $5.0 \times 10^{-4}$ & $4.5363 \times 10^{-5}$ & $1.8691 \times 10^{-6}$ \\
\hline
\end{tabular}


Tabela 7.4: Comparação entre os métodos Projeção-1 e Projeção-2, ambos utilizando a formulação AB/CN, para o erro relativo no escoamento de Hagen-Poiseuille sobre a malha M2 para $R e=0.1$.

\begin{tabular}{|c||c||c|}
\hline$\delta t(\mathrm{~s})$ & $P 1-\mathrm{AB} / \mathrm{CN}$ & $P 2-\mathrm{AB} / \mathrm{CN}$ \\
\hline $2.5 \times 10^{-5}$ & $2.7745 \times 10^{-6}$ & $1.8691 \times 10^{-6}$ \\
\hline $5.0 \times 10^{-5}$ & $3.6913 \times 10^{-6}$ & $1.8691 \times 10^{-6}$ \\
\hline $2.5 \times 10^{-4}$ & $1.6851 \times 10^{-5}$ & $1.8691 \times 10^{-6}$ \\
\hline $5.0 \times 10^{-4}$ & $4.5440 \times 10^{-5}$ & $1.8691 \times 10^{-6}$ \\
\hline
\end{tabular}

Como um dos objetivos deste trabalho é analisar a estabilidade dos métodos que utilizam formulações implícitas para resolver as equações de conservação, algumas conclusões, sobre o comportamento dos métodos Projeção-1 e Projeção-2, podem ser tiradas observando-se as figuras 7.5, 7.6 e 7.7, e as tabelas 7.2, 7.3 e 7.4. No escoamento de Hagen-Poiseuille sobre a malha M2 com $R e=0.1$, as formulações implícitas apresentaram resultados distintos, em relação à restrição de estabilidade numérica. A partir da tabela 7.2 , nota-se, o $\delta t$ máximo permitido pela formulação IR, foi igual a $1.25 \times 10^{-2} \mathrm{~s}$, determinado pela condição CFL, enquanto que para as formulações CN e $\mathrm{AB} / \mathrm{CN}$, na tabela 7.3 têm-se $\delta t=5.0 \times 10^{-4} \mathrm{~s}$. Portanto, a formulação IR com os métodos Projeção-1 e Projeção-2 foi mais estável que as formulações que utilizam o método CN. Esse fato, como discutido na seção 2.5, ocorre porque o método CN tem seu erro contaminado com oscilações numéricas e o método IR não tem. Esse fato também foi observado por (Turek, 1996).

No problema anterior com a malha M2, utilizou-se como critério de convergência na equação de Poisson a tolerância $1.0 \times 10^{-10}$. Desta forma foi possível analisar o número de iterações em cada ciclo computacional dos métodos GC e GBCP, para as formulações explícita e implícitas, respectivamente, na equação do cálculo de $\psi$. A tabela 7.5 apresenta o número de iterações e o número de equações para os tempos $t=1,6$ e 20 segundos. No tempo $t=20$ s o escoamento atingiu o estado estacionário. De acordo com os resultados da tabela 7.5 observa-se que o método GBCP convergiu para a solução com números razoáveis de iterações. Como discutido na seção 5.5 , mesmo não existindo resultados que garantam sua convergência, o método GBCP mostrou-se eficiente. Entretanto, comparado ao método GC, o GBCP demorou mais para convergir. Uma justificativa para este fato, é que o valor do $\delta t$ máximo é muito grande para as formulações implícitas que utilizam o GBCP, e desta forma, a condição inicial está muito distante da próxima solução, exigindo maior número de iterações. Nota-se também, que o sistema resultante na utilização das formulações implícitas para o cálculo de $\psi$, possui 
matriz não simétrica, enquanto que para a formulação explícita, a matriz resultante é simétrica.

Tabela 7.5: Números de equações e iterações dos métodos GC (Explícito) e GBCP (Projeção-1 e Projeção-2 utilizando as FI). Resultados para o escoamento sobre a malha M2.

\begin{tabular}{|c||c|c||c|c||c|c|}
\hline \multirow{2}{*}{ Método } & \multicolumn{2}{c|}{$t=1 \mathrm{~s}$} & \multicolumn{2}{c|}{$t=6 \mathrm{~s}$} & \multicolumn{2}{c|}{$t=20$ s } \\
\cline { 2 - 7 } & equações & iterações & equações & iterações & equações & iterações \\
\hline Explícito & 290 & 34 & 1732 & 139 & 2000 & 1 \\
\hline P1-IR & 291 & 92 & 1733 & 261 & 2000 & 1 \\
\hline P1-CN & 290 & 92 & 1732 & 279 & 2000 & 5 \\
\hline P1-AB/CN & 290 & 93 & 1732 & 286 & 2000 & 4 \\
\hline P2-IR & 291 & 134 & 1733 & 300 & 2000 & 15 \\
\hline P2-CN & 290 & 96 & 1732 & 285 & 2000 & 8 \\
\hline P2-AB/CN & 290 & 97 & 1732 & 287 & 2000 & 9 \\
\hline
\end{tabular}

Utilizando ainda o problema de Hagen-Poiseuille outras simulações foram realizadas sobre a malha intermediária, com diferentes valores de Re. Essas simulações tiveram o objetivo de analisar a estabilidade das formulações implícitas, quando o número de Re diminui, isto é, para problemas mais viscosos ou "creep flow". A tabela 7.6 mostra os resultados dos valores máximos de $\delta t$ permitidos pelas formulações implícitas e pelo método explícito. Para os valores de $\delta t$ apresentados na tabela, os métodos foram estáveis e não apresentaram oscilações. Novamente, os métodos que utilizam a formulação IR admitiram valores para $\delta t$ maiores que as outras formulações, em particular, maiores que as formulações $\mathrm{CN}$ e $\mathrm{AB} / \mathrm{CN}$. Apesar do número de $R e$ diminuir, a restrição do passo temporal para a formulação IR permaneceu a mesma, ou seja, a restriçăo é determinada pela condição CFL. Por outro lado, as formulações CN e AB/CN apresentaram uma restrição maior do que a condição $\mathrm{CFL}$, utilizando um passo temporal menor que aquela da formulação IR.

Apesar da diferença de valores de $\delta t$ das formulações implícitas, os métodos Projeção1 e Projeção-2 admitiram valores de $\delta t$ bem maiores que o método explícito, isto é, todas as formulações implícitas, como era esperado, superaram a restrição (5.22) do método explícito GENSMAC. Essa restrição, repetida aqui

$$
\delta t_{v i s c} \leq \frac{R e}{2}\left(\frac{1}{(\delta x)^{2}}+\frac{1}{(\delta y)^{2}}\right)^{-1},
$$


é imposta pela discretização explícita dos termos viscosos, como foi apresentado na subseção 5.2.4. A condição de estabilidade do método explícito, baseado na restrição (5.22), impôs severas restrições para $\delta t$, principalmente, quando o número de $R e$ diminui. Como pode ser visto na tabela 7.6, os métodos que utilizam a formulação IR, exigiram $\delta t$ cerca de 500 à 500000 vezes maiores que o método explícito, quando o número de Re diminuiu, enquanto que as formulações $\mathrm{CN}$ e $\mathrm{AB} / \mathrm{CN}$ apresentaram $\delta t$ cerca dè 20 vezes maior, independente do número de $R e$.

Tabela 7.6: Limite de estabilidade para $\delta t(\mathrm{~s})$ no escoamento de Hagen-Poiseuille sobre a malha M2, com diferentes valores para $R e$.

\begin{tabular}{|c||c||c||c||c|}
\hline Método & $R e=0.1$ & $R e=0.01$ & $R e=0.001$ & $R e=0.0001$ \\
\hline Explícito & $2.5 \times 10^{-5}$ & $2.5 \times 10^{-6}$ & $2.5 \times 10^{-7}$ & $2.5 \times 10^{-8}$ \\
\hline$P 1$-IR & $1.25 \times 10^{-2}$ & $1.25 \times 10^{-2}$ & $1.25 \times 10^{-2}$ & $1.25 \times 10^{-2}$ \\
\hline$P 1$-CN & $5.0 \times 10^{-4}$ & $5.0 \times 10^{-5}$ & $5.0 \times 10^{-6}$ & $5.0 \times 10^{-7}$ \\
\hline P1-AB/CN & $5.0 \times 10^{-4}$ & $5.0 \times 10^{-5}$ & $5.0 \times 10^{-6}$ & $5.0 \times 10^{-7}$ \\
\hline P2-IR & $1.25 \times 10^{-2}$ & $1.25 \times 10^{-2}$ & $1.25 \times 10^{-2}$ & $1.25 \times 10^{-2}$ \\
\hline P2-CN & $5.0 \times 10^{-4}$ & $5.0 \times 10^{-5}$ & $5.0 \times 10^{-6}$ & $5.0 \times 10^{-7}$ \\
\hline P2-AB/CN & $5.0 \times 10^{-4}$ & $5.0 \times 10^{-5}$ & $5.0 \times 10^{-6}$ & $5.0 \times 10^{-7}$ \\
\hline
\end{tabular}

Outro objetivo do presente trabalho, é a comparação dos métodos Projeção- 1 e Projeção-2 utilizando as FI com o método explícito original baseado na metodologia GENSMAC. As características mais importantes para a comparação entre esses métodos, foram o valor do erro relativo, o tamanho do passo temporal permitido por cađa método e o tempo de processamento. Para o mesmo problema do escoamento de Hagen-Poiseuille sobre a malha intermediária, simulações foram realizadas, comparando a performance do método explícito e dos métodos que utilizam formulações implícitas. Esses resultados são apresentados nas tabelas 7.7, 7.8 e 7.9. Os valores para $\delta t$ utilizado pelas FI, foram escolhidos de forma que o erro relativo desses métodos, não desvia-se significativamente do erro do método explícito. Portanto, não se usou um $\delta t$ muito grande, para que a precisão numérica não fosse afetada. Como foi discutido na seção 4.3, ao aplicar formulações implícitas, que permitem o uso do passo temporal bem maior que o do método explícito (como foi mostrado na tabela 7.6), deve-se tomar cuidado para que os resultados obtidos por essas formulações não sejam imprecisos.

Na tabela 7.7, observa-se que o método Projeção-2 utilizando a formulação IR consumiu um tempo de processamento aproximadamente 20 vezes menor que o método explícito. Os outros métodos foram aproximadamente 4 vezes mais rápidos que o método explícito. Nota-se pelas tabelas 7.8 e 7.9, que o método Projeção-2 utilizando a formu- 
lação IR, continuou sendo muito mais rápido que os outros métodos, com boa precisão numérica, quando o número de $R e$ diminui. Em comparação com o método explícito, todos os métodos que utilizaram FI, foram significativamente mais rápidos.

A comparação do tempo de processamento entre os métodos que utilizam FI e o método explícito, é o ponto fundamental deste trabalho. Como observado nas tabelas 7.7, 7.8 e 7.9, os métodos Projeção-1 e Projeção-2 utilizando as FI, produzem a solução exata como o método explícito, mas com uma redução importante no tempo de processamento.

Nota-se que os resultados obtidos pelo esquema preditor-corretor $\mathrm{AB} / \mathrm{CN}$ no método Projeção-2 foram muito próximos à formulação CN na Projeção-2. Essse fato era esperado, pois o objetivo deste trabalho é analisar problemas em simulações onde o valor do número de Reynolds é baixo, e desta forma os termos convectivos não têm forte influência no escoamento.

Tabela 7.7: Resultados para o escoamento de Hagen-Poiseuille para $R e=0.1$, sobre a malha M2, com tempo de simulação $t=20$ s.

\begin{tabular}{|c||c||c||c|}
\hline Método & $E_{\text {rel }}$ & $\delta t(\mathrm{~s})$ & Tempo de processamento-(m:s) \\
\hline Explícito & $1.8691 \times 10^{-6}$ & $2.5 \times 10^{-5}$ & $104: 40$ \\
\hline$P 1$-IR & $3.6879 \times 10^{-5}$ & $2.5 \times 10^{-4}$ & $25: 08$ \\
\hline P1-CN & $4.5363 \times 10^{-5}$ & $5.0 \times 10^{-4}$ & $22: 41$ \\
\hline$P 1$-AB/CN & $4.5363 \times 10^{-5}$ & $5.0 \times 10^{-4}$ & $30: 41$ \\
\hline P2-IR & $1.8689 \times 10^{-6}$ & $1.25 \times 10^{-2}$ & $5: 20$ \\
\hline P2-CN & $1.8691 \times 10^{-6}$ & $5.0 \times 10^{-4}$ & $24: 01$ \\
\hline P2-AB/CN & $1.8691 \times 10^{-6}$ & $5.0 \times 10^{-4}$ & $25: 29$ \\
\hline
\end{tabular}

Tabela 7.8: Resultados para o escoamento de Hagen-Poiseuille para $R e=0.01$, sobre a malha M2, com tempo de simulação $t=20 \mathrm{~s}$.

\begin{tabular}{|c||c||c||c|}
\hline Método & $E_{\text {rel }}$ & $\delta t(\mathrm{~s})$ & Tempo de processamento-(m:s) \\
\hline Explícito & $1.8691 \times 10^{-6}$ & $2.5 \times 10^{-6}$ & $875: 16$ \\
\hline$P 1$-IR & $3.5371 \times 10^{-5}$ & $2.5 \times 10^{-5}$ & $230: 11$ \\
\hline$P 1$-CN & $4.5363 \times 10^{-5}$ & $5.0 \times 10^{-5}$ & $151: 04$ \\
\hline$P 1$-AB/CN & $4.5363 \times 10^{-5}$ & $5.0 \times 10^{-5}$ & $245: 01$ \\
\hline$P 2-I R$ & $1.8691 \times 10^{-6}$ & $1.0 \times 10^{-3}$ & $18: 17$ \\
\hline P2-CN & $1.8691 \times 10^{-6}$ & $5.0 \times 10^{-5}$ & $164: 15$ \\
\hline$P^{2}$-AB/CN & $1.8691 \times 10^{-6}$ & $5.0 \times 10^{-5}$ & $168: 47$ \\
\hline
\end{tabular}


Tabela 7.9: Resultados para o escoamento de Hagen-Poiseuille para $R e=0.001$, sobre a malha M2, com tempo de simulação $t=30$ s.

\begin{tabular}{|c||c||c||c|}
\hline Método & $E_{\text {rel }}$ & $\delta t(\mathrm{~s})$ & Tempo de processamento-(m:s) \\
\hline Explícito & $1.8691 \times 10^{-6}$ & $2.5 \times 10^{-7}$ & $7054: 50$ \\
\hline P1-IR & $3.4147 \times 10^{-5}$ & $2.5 \times 10^{-4}$ & $1541: 06$ \\
\hline P1-CN & $3.9853 \times 10^{-5}$ & $5.0 \times 10^{-6}$ & $1143: 59$ \\
\hline P1-AB/CN & $3.9853 \times 10^{-5}$ & $5.0 \times 10^{-6}$ & $1231: 59$ \\
\hline P2-IR & $1.8689 \times 10^{-6}$ & $3.25 \times 10^{-3}$ & $31: 43$ \\
\hline P2-CN & $1.8691 \times 10^{-6}$ & $5.0 \times 10^{-6}$ & $1171: 02$ \\
\hline P2-AB/CN & $1.8691 \times 10^{-6}$ & $5.0 \times 10^{-6}$ & $1183: 19$ \\
\hline
\end{tabular}

\subsection{Comparação com a Solução Aproximada de Wat-} son

Apesar, do objetivo principal deste trabalho, consistir em resolver escoamentos com superfícies livres com baixo número de Reynolds aplicando técnicas implícitas, testes foram realizados em problemas com número de Reynolds elevado. Um problema nesse regime foi proposto por (Watson, 1964) e permite a análise e validação do comportamento da superfície livre. De acordo com (Watson, 1964), esse problema consiste no escoamento de um fluido incidindo sobre uma superfície rígida e impermeável (ou seja, sem poros), sob o efeito do campo gravitacional. Na sua análise, Watson desprezou os efeitos de tensão superficial, e examinou o comportamento da superfície livre, isto é, analisou a altura $y_{s}$ da superfície rígida até a superfície livre, após o escoamento atingir o estado estacionário. Apresentam-se, resumidamente a seguir, a solução analítica de Watson, para o comportamento da altura $y_{s}$.

De acordo com Watson, a altura é dada pela expressão

$$
y_{s}\left(x_{A}\right)=\frac{\pi}{\sqrt{3}} \frac{\nu\left(x_{A}+l\right)}{Q}
$$

onde $x_{A}$ é a coordenada $x$ adimensionalizada, $l$ é uma constante definida por

$$
l=\left(\frac{3 \sqrt{3}(1.402)(2 \sqrt{3}(1.402)-\pi)}{2 \pi^{2}}\right) L R e,
$$

e $Q$ é a vazão definida como

$$
Q=\left(\frac{\bar{L}}{2}\right) U_{0}
$$


$L$ é o diâmetro do injetor e $U_{0}$ a velocidade de injeção. Mais detalhes sobre a descrição da solução aproximada de Watson podem ser encontrados em (Watson, 1964) e (Ferreira et al., 2002).

O propósito desta seção é mostrar os resultados numéricos obtidos pelos métodos Projeção-1 e Projeção-2 utilizando as FI, em comparação com a solução aproximada de Watson, verificando o comportamento da superfície livre. Outra verificação importante é mostrar a eficiência desses métodos para problemas com número de Reynolds moderados. Para esta simulação, $R e=5000$. O domínio para este problema é dado na figura 7.8 .

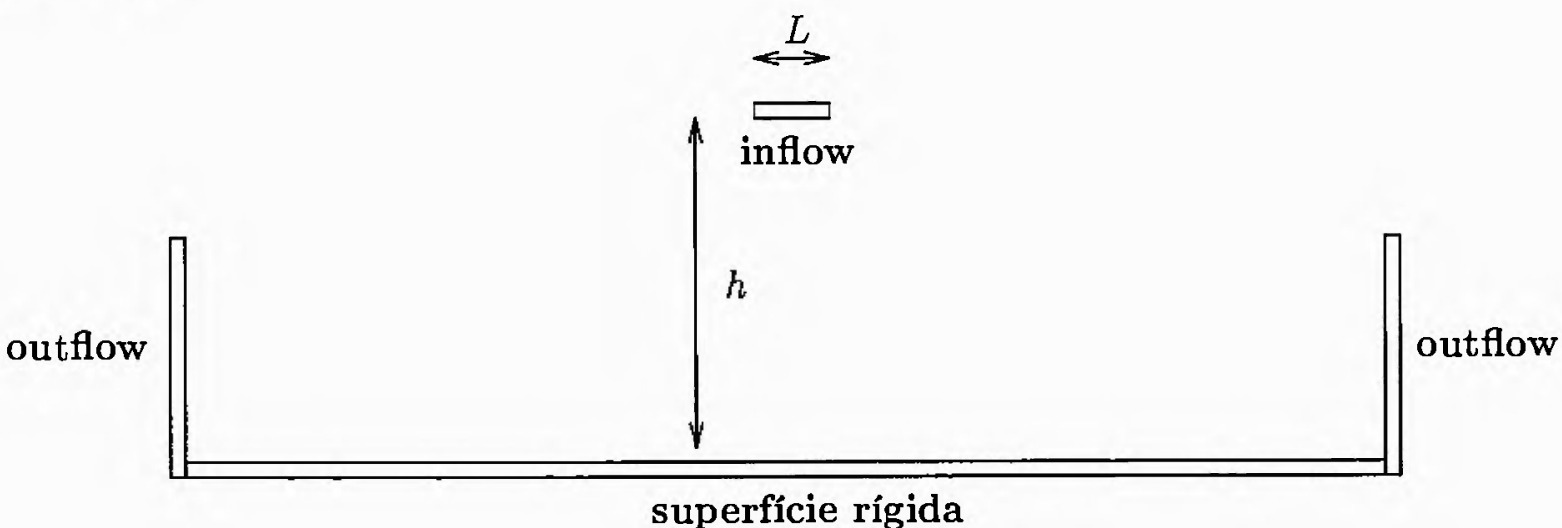

Figura 7.8: Definição do domínio para o escoamento de um jato livre sobre uma superfície rígida impermeável.

As comparações entre as soluções obtidas pelos métodos Projeção-1 e Projeção-2 utilizando as FI e a solução aproximada de Watson foram implementadas para simular o problema em questão, aplicando-se os seguintes parâmetros:

- Dimensão do domínio: $0.4 \mathrm{~m} \times 0.04 \mathrm{~m}$;

- Diâmetro do injetor $(L): 0.01 \mathrm{~m}$;

- Velocidade de injeção $(U): 1.0 \mathrm{~ms}^{-1}$;

- Coeficiente de viscosidade cinemática $(\nu): 0.000002 \mathrm{~m}^{2} \mathrm{~s}^{-1}$;

- Parâmetros de escala: $L=0.01 \mathrm{~m}$ e $U=1.0 \mathrm{~ms}^{-1}$;

- Altura do injetor a partir da superfície rígida $(h): 0.037 \mathrm{~m}$; 
- Número de Reynolds $(R e=U L / \nu): 5000$;

- Número de Froude $(F r=U / \sqrt{g} L): 3.19275$.

Uma ilustração do escoamento de um jato sobre uma superfície rígida impermeável pode ser visto na figura 7.9 , que mostra a superfície livre do fluido.

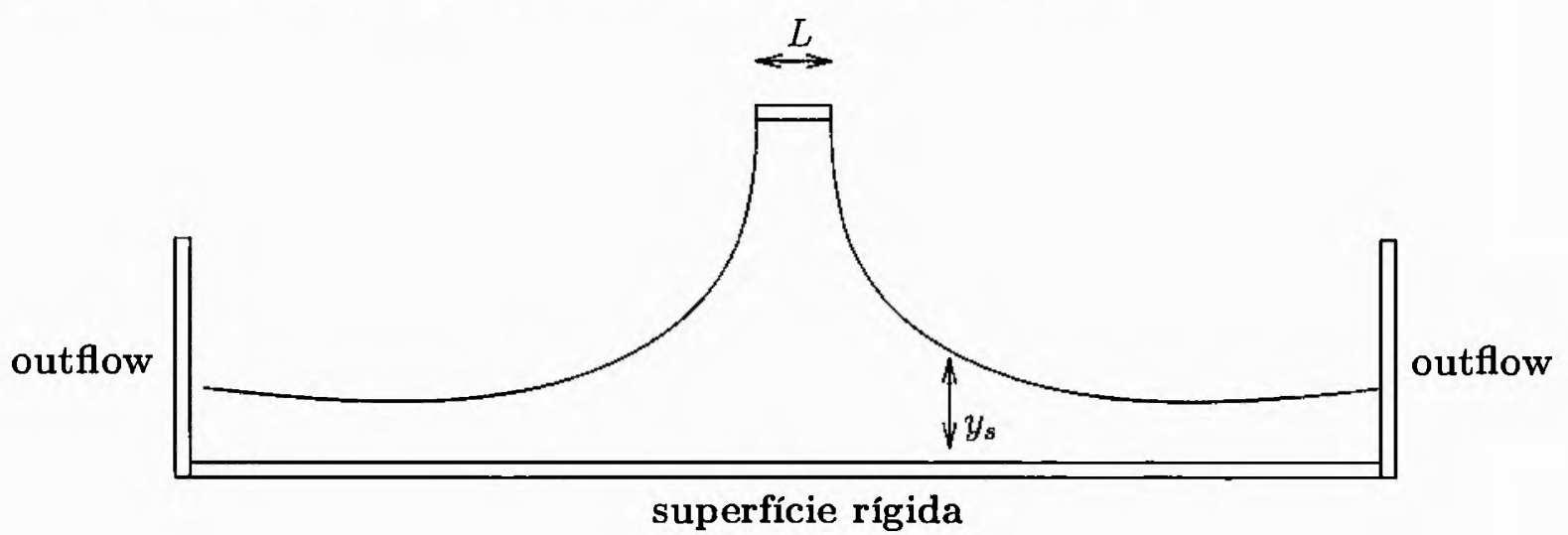

Figura 7.9: Superfície livre de um jato de fluido incidindo sobre uma superfície rígida impermeável.

A malha utilizada nessa simulação foi de $800 \times 80$ células computacionais $\left(\delta_{x}=\right.$ $\left.\delta_{y}=0.00050 \mathrm{~m}\right)$. Uma comparação entre as soluções numéricas e a solução analítica de Watson (Watson, 1964) é apresentada na figura 7.10 e 7.11.

Estas figuras mostram a superfície livre do fluido adimensional $(h / 0.5 L)$ contra a distância adimensional $(x / 0.5 L) R e^{-1}$. Esses resultados foram obtidos após o tempo $t=4.0 \mathrm{~s}$. Pode-se observar nas figuras 7.10 e 7.11 que os resultados numéricos para os método Projeção-1 e Projeção-2 utilizando as formulações implícitas estão bem próximos da solução analítica de Watson, apresentando uma ligeira discrepância. Atribui-se esse fato ao argumento de que a solução analítica de Watson é somente uma aproximação. Nota-se também, que para este problema, os resultados numéricos obtido pelos métodos Projeção-1 e Projeção-2 são muito parecidos. Como o número de $R e=5000$, o valor de $\delta t$ utilizado pelas formulações implícitas, não foi muito superior àquele do método explícito, pois a condição de estabilidade (5.22), tem o número de Reynolds como fator, não restringindo demais o passo temporal para o método explícito. $O$ valor do $\delta t$ permitido pelo método explícito foi $1.5625 \times 10^{-5} \mathrm{~s}$, enquanto que para os métodos Projeção-1 e Projeção-2 utilizando as FI, a restrição da estabilidade foi baseada na 
a)

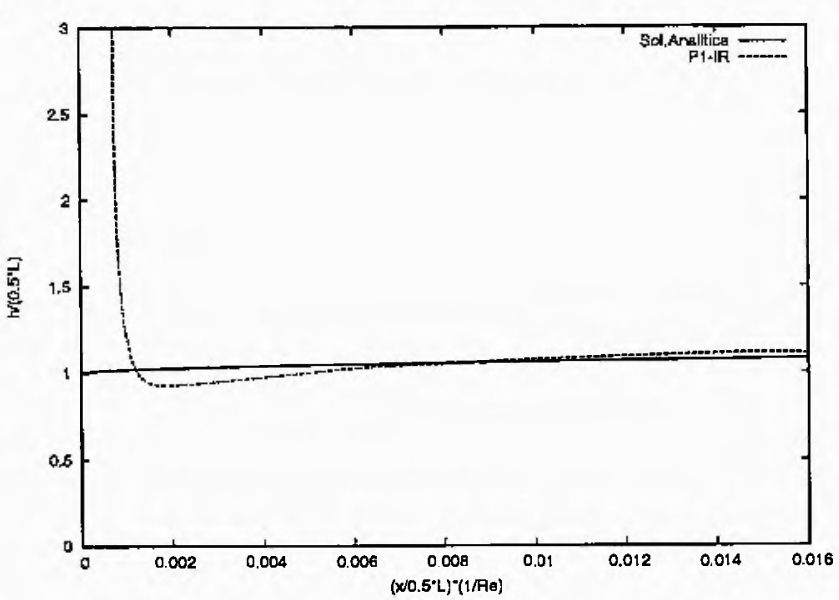

b)

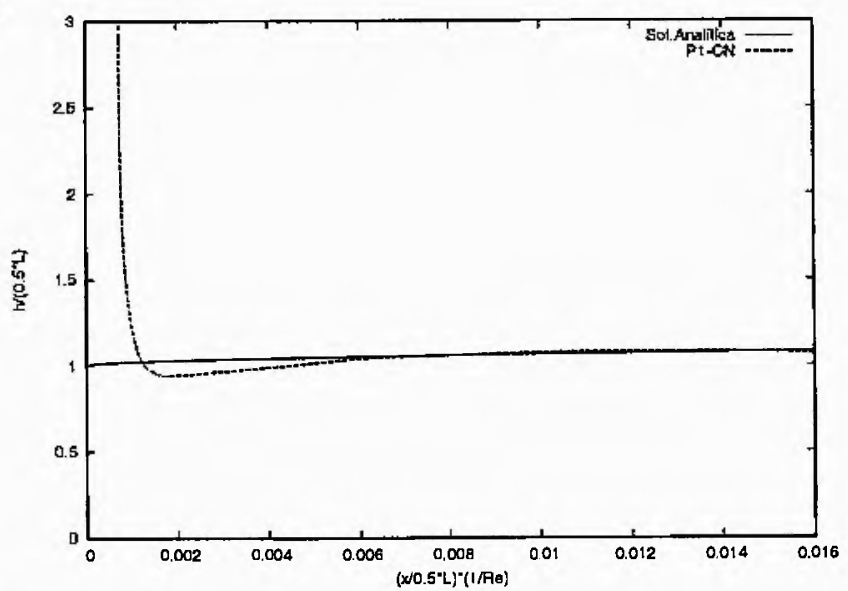

c)

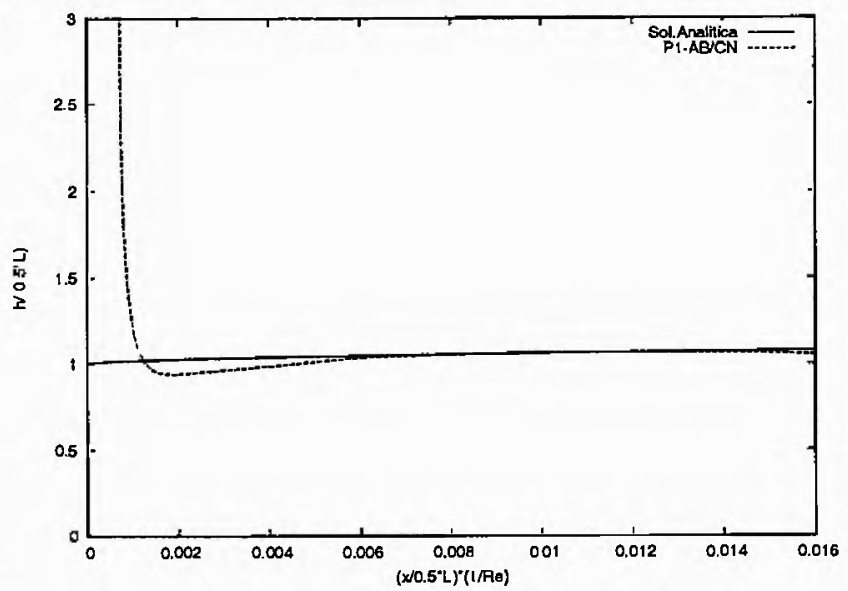

Figura 7.10: Comparação entre soluções numéricas obtida pelo método Projeção-1 utilizando FI e a solução analítica de Watson, com $R e=5000$. As figuras a)-c) correspondem ao método Projeçẫo-1 utilizando as formulações IR, CN e AB/CN, respectivamente. 
a)

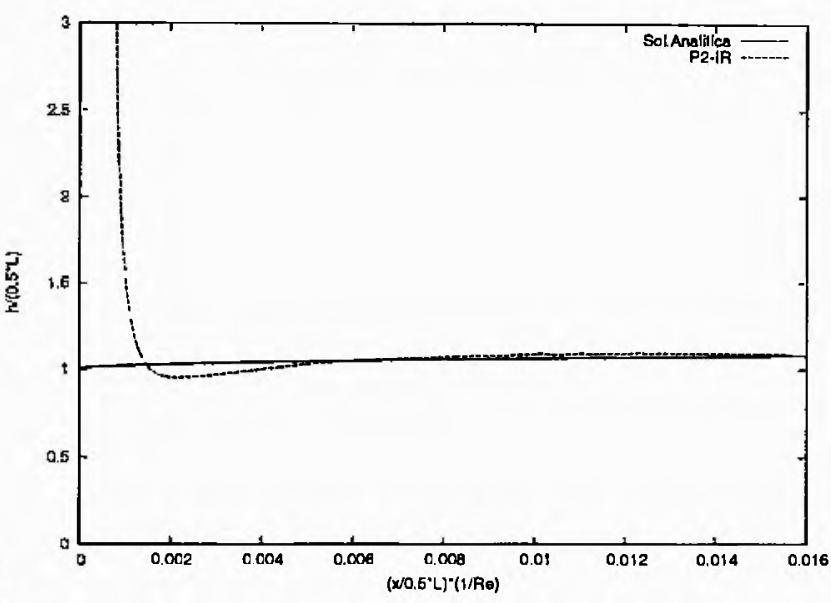

b)

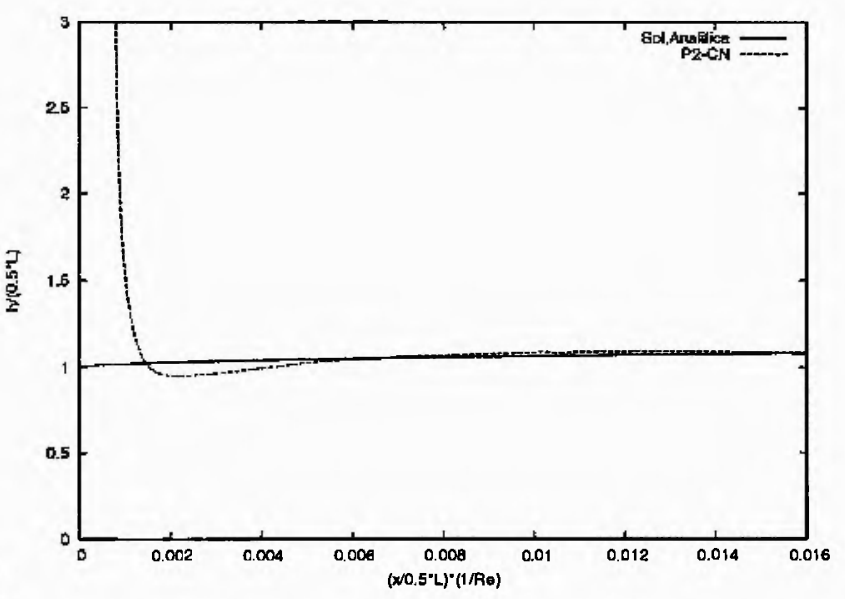

c)

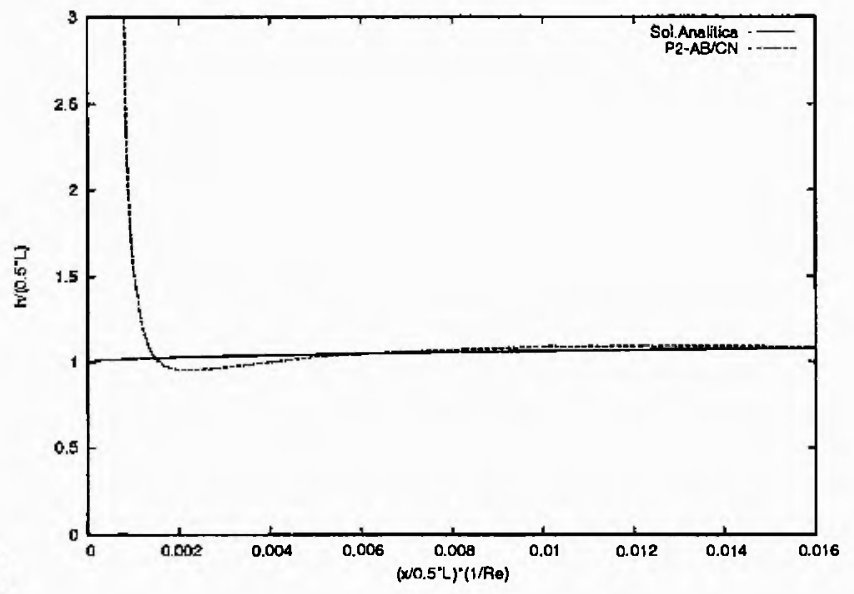

Figura 7.11: Comparação entre soluções numéricas obtida pelo método Projeção-2 utilizando FI e a solução analítica de Watson, com $R e=5000$. As figuras a)-c) correspondem ao método Projeção-2 utilizando as formulações IR, CN e AB/CN, respectivamente. 
condição CFL, determinando $\delta t=6.0 \times 10^{-5}$ s. Para esse problema, as formulações implícitas IR, $\mathrm{CN}$ e $\mathrm{AB} / \mathrm{CN}$, permitiram o mesmo $\delta t$.

\subsection{Simulação Numérica do Preenchimento de uma Caixa}

Nesta seção mostram-se os resultados numéricos utilizando os métodos Projeção-1 e Projeção-2 com as FI, para a simulação do preenchimento de uma caixa por um fluido newtoniano com $R e=0.1$ e $R e=0.01$. O domínio utilizado pode ser visto na figura 7.12. Para as simulações numéricas dos métodos Projeção-1 e Projeção-2 utilizando as

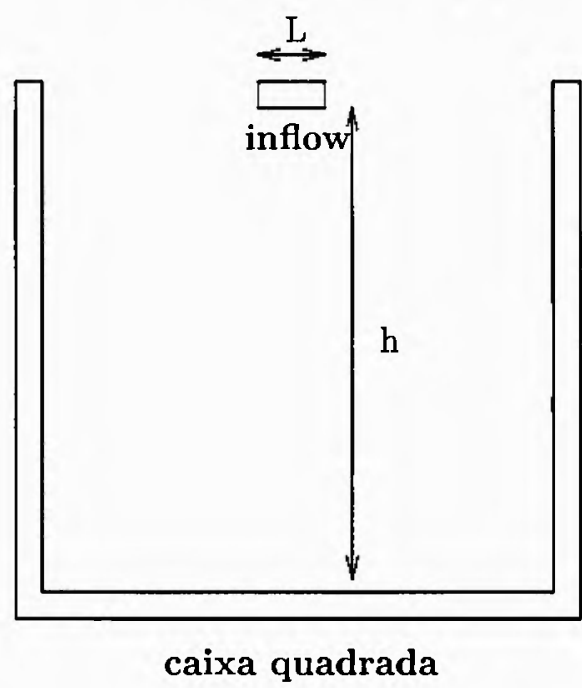

Figura 7.12: Domínio para o problema do preenchimento de uma caixa.

FI, dois modelos foram empregados:

Modelo $1-R e=0.1$

- Dimensão do domínio: $0.05 \mathrm{~m} \times 0.05 \mathrm{~m}$;

- Diâmetro do injetor $(L): 0.005 \mathrm{~m}$;

- Velocidade de injeção $(U): 1.0 \mathrm{~ms}^{-1}$;

- Coeficiente de viscosidade cinemática $(\nu): 0.05 \mathrm{~m}^{2} \mathrm{~s}^{-1}$; 
- Parâmetros de escala: $L=0.005 \mathrm{~m}$ e $U=1.0 \mathrm{~ms}^{-1}$;

- Altura do injetor a partir da superfície rígida $(h): 0.047 \mathrm{~m}$;

- Número de Reynolds ( $R e=U L / \nu)$ : 0.1;

- Número de Froude $(F r=U / \sqrt{g} L): 4.5152$.

Modelo $2-R e=0.01$

Mesmos parâmetros do Modelo 1, a menos de:

- Coeficiente de viscosidade cinemática $(\nu): 0.5 \mathrm{~m}^{2} \mathrm{~s}^{-1}$;

- Número de Reynolds $(R e=U L / \nu): 0.01$.

Nestas simulações, uma malha de $100 \times 100$ células computacionais $\left(\delta_{x}=\delta_{y}=\right.$ $0.00050 \mathrm{~m}$ ) foi utilizada para todos os métodos. Nesses dois modelos, o campo gravitacional age sobre o escoamento e o tempo final das simulaçôes foi $t=5 \mathrm{~s}$.

Um efeito muito conhecido na literatura, que ocorre para escoamentos de um jato de fluidos newtonianos, em determinadas circunstâncias, é o efeito de "fiambagem" ou efeito "buckling". Os resultados experimentais foram publicados por (Cruickshank e Munson, 1981), concluindo que a "fiambagem", ocorre quando as seguintes restrições são satisfeitas

$$
R e<0.56 \text { e } \frac{h}{L}>3 \pi
$$

onde $h$ é a altura do injetor até a superfície rígida e $L$ o diâmetro do injetor.

Para os Modelo $1-R e=0.1$ e Modelo $2-R e=0.01, \frac{h}{L}=\frac{0.047}{0.005}=9.4<$ $3 \pi$, ou seja, de acordo com a restrição (7.17b), esses modelos devem resultar em um escoamento sem flambagens. As simulações numéricas podem ser vistas na figura 7.13. Note que a solução numérica do método explícito também foi apresentada, para efeito de comparação. Observa-se na figura 7.13a), que o método explícito, apresenta uma pequena fiambagem, enquanto que os métodos Projeção-1 e Projeção-2 utilizando as FI, não apresentam esse efeito, e têm resultados praticamente iguais (figuras $7.13 \mathrm{~b}$ )-g)). Como comentado acima, nas condições desse escoamento, o efeito de fiambagem não deveria ocorrer, mas o método explícito apresenta esse fenômeno. Essa observação pode ser explicado pelo fato de as restrições (7.17) terem sido obtidas experimentalmente, $\mathrm{e}$ o método explícito foi mais sensível às restrições, já que a restrição $\frac{h}{L}=9.4$ é muito próxima de $3 \pi \simeq 9.43$. 

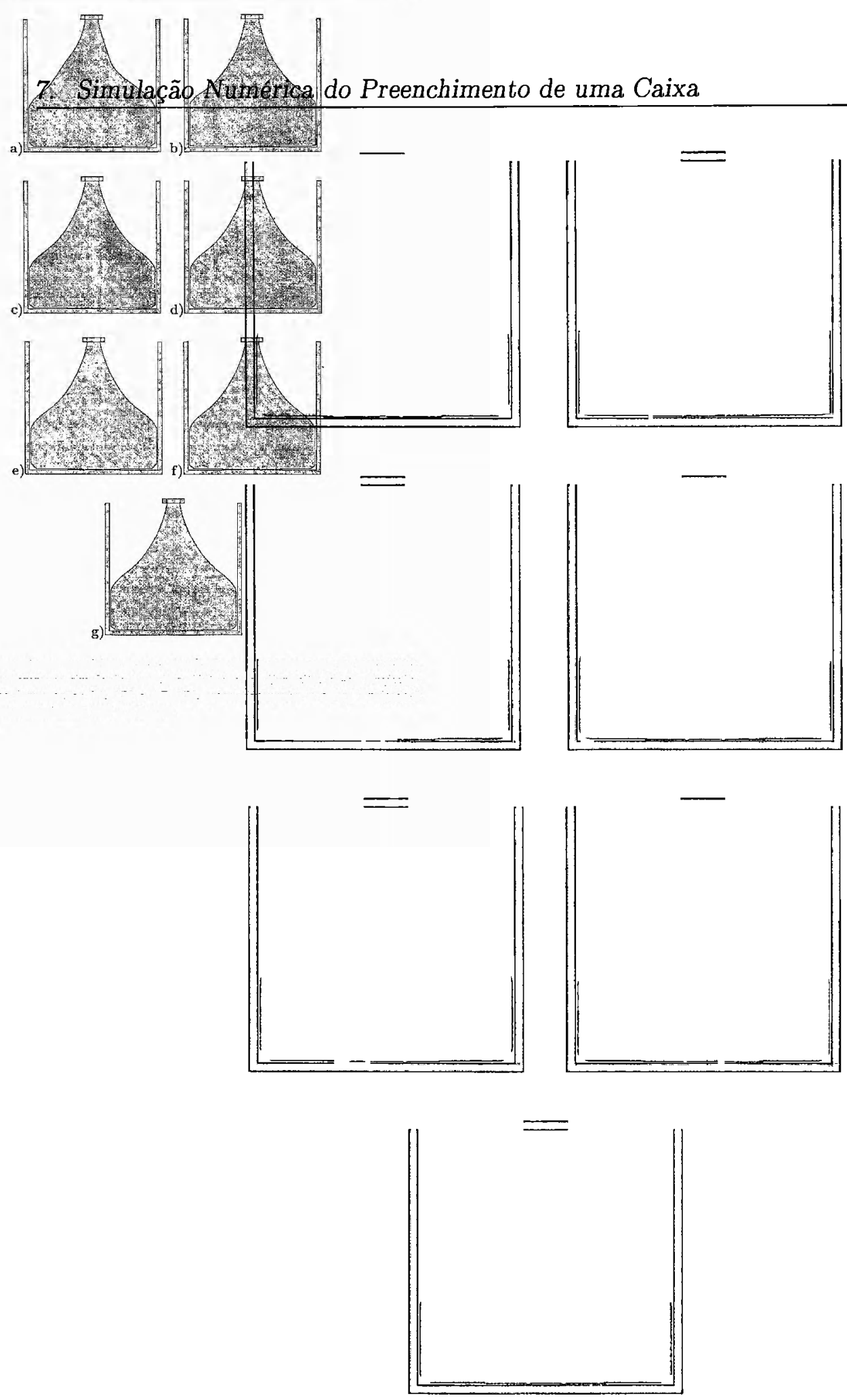

Figura 7.13: Resultados numéricos para a simulação do preenchimento de uma caixa, com $R e-0.1$ e tempo de simulação $t=0.28 \mathrm{~s}$. Métodos: a) Explícito, b)-d) Projeção1 utilizando as formulações $\mathrm{IR}, \mathrm{CN}$ e $\mathrm{AB} / \mathrm{CN}$, respectivamente, e e)-g) Projeção-2 utilizando as formulações IR, $\mathrm{CN}$ e $\mathrm{AB} / \mathrm{CN}$. 
Tabela 7.10: Resultados para o escoamento de um jato preenchendo um caixa de $L=$ $0.05 \mathrm{~m}, U=1.0 \mathrm{~ms}^{-1}, R e=0.1$ no instante $t=0.28 \mathrm{~s}$.

\begin{tabular}{|c||c||c||c|}
\hline Método & $\delta t(\mathrm{~s})$ & Número de iterações & Tempo de processamento-(m:s) \\
\hline Explícito & $5.0 \times 10^{-7}$ & 559998 & $430: 59$ \\
\hline$P 1$-IR & $3.0 \times 10^{-5}$ & 11200 & $41: 52$ \\
\hline$P 1$-CN & $1.0 \times 10^{-5}$ & 28000 & $99: 16$ \\
\hline$P 1$-AB/CN & $1.0 \times 10^{-5}$ & 28000 & $106: 18$ \\
\hline$P 2$-IR & $6.0 \times 10^{-5}$ & 8960 & $21: 41$ \\
\hline P2-CN & $1.0 \times 10^{-5}$ & 28000 & $92: 51$ \\
\hline P2-AB/CN & $1.0 \times 10^{-5}$ & 28000 & $96: 25$ \\
\hline
\end{tabular}

Tabela 7.11: Resultados para o escoamento de um jato preenchendo um caixa de $L-$ $0.05 \mathrm{~m}, U=1.0 \mathrm{~ms}^{-1}, R e=0.01$ no instante $t=0.28 \mathrm{~s}$.

\begin{tabular}{|c||c||c||c|}
\hline Método & $\delta t(\mathrm{~s})$ & Número de iterações & Tempo de processamento-(m:s) \\
\hline Explícito & $5.0 \times 10^{-8}$ & 5599980 & $4326: 43$ \\
\hline$P 1$-IR & $3.0 \times 10^{-6}$ & 112000 & $738: 23$ \\
\hline$P 1$-CN & $1.0 \times 10^{-6}$ & 280000 & $1108: 57$ \\
\hline$P 1$-AB/CN & $1.0 \times 10^{-6}$ & 280000 & $1149: 01$ \\
\hline$P 2-$ IR & $6.0 \times 10^{-5}$ & 8960 & $54: 51$ \\
\hline$P 2$-CN & $1.0 \times 10^{-6}$ & 280000 & $1047: 44$ \\
\hline P2-AB/CN & $1.0 \times 10^{-6}$ & 280000 & $1059: 18$ \\
\hline
\end{tabular}

Mais uma vez foram realizadas comparações entre os métodos que utilizam as formulações implícitas e explícita, verificando o valor de $\delta t$ permitido para cada método, o número de iterações e o tempo de processamento, para o tempo de simulação $t=0.28 \mathrm{~s}$. Os resultados apresentados na tabela 7.10 foram obtidos para a simulação do Modelo 1 - $R e=0.1$, e a tabela 7.11, para o Modelo 2 - $R e-0.01$. Novamente, as formulações implícitas superaram a restrição (5.22) do método explícito, como era esperado, e utilizaram menos iterações para se obter a solução no tempo $t=0.28 \mathrm{~s}$. Com relação ao tempo de processamento, os métodos Projeção-1 e Projeção-2 utilizando as FI foram bem mais rápidos que o método explícito, determinando a solução em $t-0.28 \mathrm{~s}$. Em particular, o método Projeção-2 utilizando a formulação IR, apresentou os melhores resultados de estabilidade entre as formulações implícitas, pois o valor de $\delta t$ foi restrito apenas pela condição CFL. Para o primeiro modelo, o passo temporal para este método foi 120 vezes maior do que aquele do método explícito, enquanto que para o segundo modelo, 1200 vezes maior. Os resultados comparativos podem ser visto nas tabelas 7.10 e 7.11 . 


\subsection{Simulação Numérica do Preenchimento de uma Caixa contendo Fluido}

Nesta seção, os métodos Projeção-1 e Projeção-2 com as FI são aplicados para a simulação do preenchimento de uma caixa contendo um fluido. O fluido que está na caixa e o que está saindo do injetor são newtonianos com $R e=0.22$. O domínio da caixa contendo fluido pode ser visto na figura 7.14.

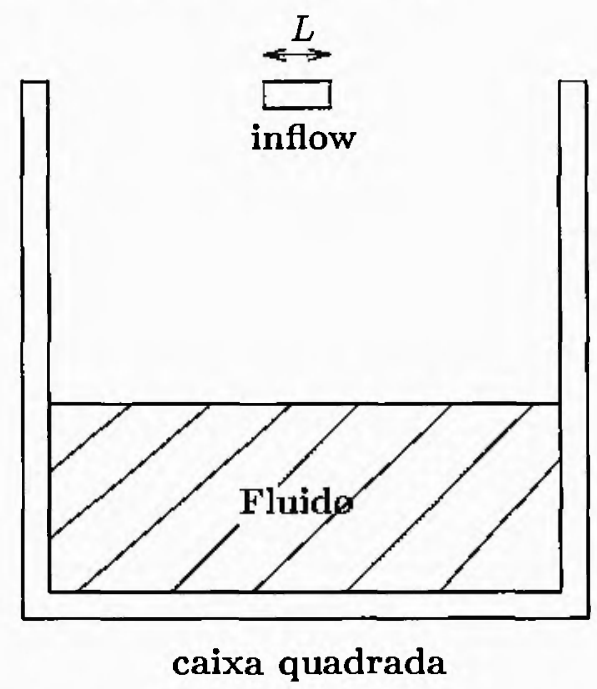

Figura 7.14: Domínio para o problema do preenchimento de uma caixa contendo fluido.

O modelo empregados nessa simulação é:

Modelo $1-R e=0.22$

- Dimensão do domínio: $0.026 \mathrm{~m} \times 0.028 \mathrm{~m}$;

- Diâmetro do injetor $(L): 0.002 \mathrm{~m}$;

- Velocidade de injeção $(U): 1.0 \mathrm{~ms}^{-1}$;

- Coeficiente de viscosidade cinemática $(\nu): 0.0087 \mathrm{~m}^{2} \mathrm{~s}^{-1}$;

- Parâmetros de escala: $L=0.002 \mathrm{~m}$ e $U=1.0 \mathrm{~ms}^{-1}$;

- Número de Reynolds $(R e=U L / \nu)$ : 0.22; 
- Número de Froude $(F r=U / \sqrt{g} L): 7.1392$.

Uma malha de $52 \times 56$ células computacionais $\left(\delta_{x}=\delta_{y}=0.00050 \mathrm{~m}\right)$ foi utilizada para todos os métodos com o campo gravitacional agindo sobre o escoamento. $\mathrm{Na}$ figura 7.15, mostra-se a comparação entre os métodos explícito e Projeção-2 utilizando a formulação IR para a pressão $p$. Nota-se que o resultado obtido pela Projeção-2 utilizando a formulação IR está em boa concordância com o resultado do método explícito. Como os resultados obtidos pelo método Projeção-1 utilizando as formulações IR, CN e $\mathrm{AB} / \mathrm{CN}$ e o método Projeção-2 utilizando as formulações $\mathrm{CN}$ e $\mathrm{AB} / \mathrm{CN}$ foram muito próximos daquele do método Projeção-2 utilizando a formulação IR, apenas um desses resultados foi apresentado na figura 7.15 .

a)

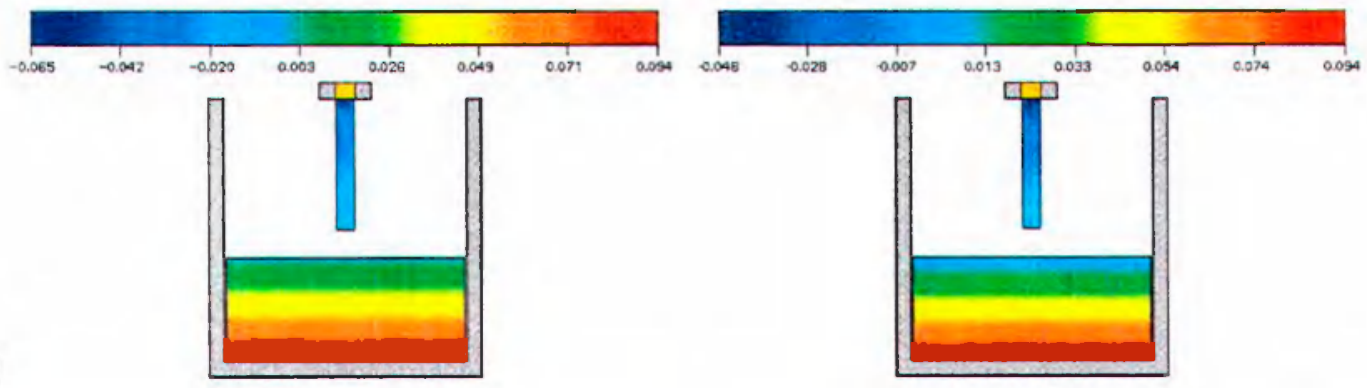

b)

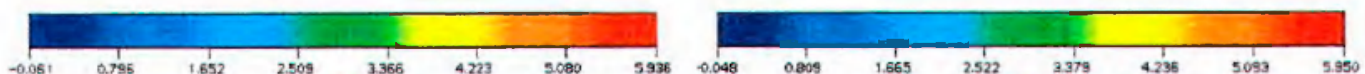

Figura 7.15: Comparação da pressão $p$ para o Modelo 1 - $R e=0.22$ entre as soluções do método explícito (esquerda) e Projeção-2 utilizando a formulação IR (direita). a) $t=0.0125 \mathrm{~s} \mathrm{e} \mathrm{b)} t=0.1375 \mathrm{~s}$.

A tabela 7.12 apresenta o valor de $\delta t$ permitido pelas formulações explícita e implícitas mostrando mais uma vez a eficiência dos métodos Projeção-1 e Projeção-2 utilizando as FI, pois superaram a restrição do método explícito. O $\delta t$ da Projeção-2 utilizando a formulação IR foi determinado pela condição CFL. Também nessa tabela apresenta-se o número de iterações de cada método para dois tempos da simulação. 
Tabela 7.12: Modelo $1-R e=0.22, L=0.002 \mathrm{~m}$ e $U=1.0 \mathrm{~ms}^{-1}$.

\begin{tabular}{|c|c|c|c|}
\hline Método & $\delta t(\mathrm{~s})$ & \multicolumn{2}{|c|}{ Número de iterações } \\
\cline { 3 - 4 } & & $t=0.0125 \mathrm{~s}$ & $t=0.1375 \mathrm{~s}$ \\
\hline Explícito & $1.8 \times 10^{-6}$ & 6960 & 76560 \\
\hline$P 1$-IR & $3.6 \times 10^{-5}$ & 348 & 3828 \\
\hline$P 1$-CN & $5.0 \times 10^{-5}$ & 218 & 2393 \\
\hline$P 1$-AB/CN & $5.0 \times 10^{-5}$ & 218 & 2393 \\
\hline$P 2-\mathrm{IR}$ & $1.8 \times 10^{-4}$ & 70 & 766 \\
\hline P2-CN & $5.0 \times 10^{-5}$ & 218 & 2394 \\
\hline P2-AB/CN & $5.0 \times 10^{-5}$ & 218 & 2393 \\
\hline
\end{tabular}

\subsection{Comparação com Resultados Experimentais}

Esse capítulo é finalizado apresentando-se comparações qualitativas das simulações numéricas com os experimentos descritos em (Tomé et al., 1999). Dois escoamentos são apresentados para a comparação utilizando o domínio descrito na figura 7.12. O método explícito também é utilizado novamente, para comparações de tempo e resultados com os métodos que utilizam as FI. Para as comparações com as visualizações dos experimentos de (Tomé et al., 1999), utiliza-se o primeiro modelo, descrito a seguir

Modelo $1-R e=0.8$

- Dimensão do domínio: $0.05 \mathrm{~m} \times 0.05 \mathrm{~m}$;

- Diâmetro do injetor $(L): 0.004 \mathrm{~m}$;

- Velocidade de injeção $(U): 1.0 \mathrm{~ms}^{-1}$;

- Coeficiente de viscosidade cinemática $(\nu): 0.005 \mathrm{~m}^{2} \mathrm{~s}^{-1}$;

- Parâmetros de escala: $L=0.004 \mathrm{~m}$ e $U=1.0 \mathrm{~ms}^{-1}$;

- Altura do injetor a partir da superfície rígida $(h)$ : $0.047 \mathrm{~m}$;

- Número de Reynolds $(\operatorname{Re}=U L / \nu)$ : 0.8;

- Número de Froude $(F r=U / \sqrt{g} L): 5.0481$.

Para este modelo, uma malha de $100 \times 100$ células computacionais $\left(\delta_{x}=\delta_{y}=\right.$ $0.00050 \mathrm{~m}$ ) foi utilizada para todos os métodos, com o campo gravitacional agindo sobre o escoamento e o tempo final das simulações foi $t=5 \mathrm{~s}$. As figuras apresentadas na 
seqüência ilustram as comparações entre as simulações e os experimentos de (Tomé et al., 1999), mostrando, qualitativamente, concordância satisfatória.

A figura 7.16 apresenta a comparação entre as soluções numéricas e o experimento. Nesta figura, os métodos numéricos apresentados foram o método Projeção-2 utilizando a formulação IR e o método explícito. Os outros métodos que utilizam as formulações implícitas, não são apresentados comparados com o resultado experimental de (Tomé et al., 1999), pois apresentaram resultados iguais ao método Projeção-2 utilizando a formulação IR.

Nas figuras 7.17 e 7.18 são apresentadas comparações entre o método explícito e os métodos Projeção-1 e Projeção-2 utilizando as FI, para as velocidades $u$ e $v$, respectivamente. Os métodos apresentaram soluções próximas, apesar da diferença no valor de $\delta t$, como observado nas figuras 7.17 e 7.18. As formulações implícitas, de acordo com a tabela 7.13 apresentaram, como anteriormente, valores maiores para $\delta t$, superando a restrição do método explícito descrito por (Tomé et al., 1999). No caso dos métodos que utilizam a formulação IR, o valor de $\delta t$ foi aproximadamente 27 vezes maior do que o permitido pelo método explícito, sendo determinado pela condição CFL. Para os métodos que utilizam as formulações $\mathrm{CN}$ e $\mathrm{AB} / \mathrm{CN}$, o valor de $\delta t$ foi 20 vezes maior que o do método explícito. Essa diferença nos valores de $\delta t$ dos métodos Projeção-1 e Projeção-2 utilizando as FI, é mais representativa comparando-se o número de iterações para cada tempo descrito na figura 7.16, o que é apresentado na tabela 7.13, quando comparado com o método explícito.

Tabela 7.13: Modelo $1-R e=0.8, L=0.004 \mathrm{~m}$ e $U=1.0 \mathrm{~ms}^{-1}$.

\begin{tabular}{|c|c|c|c|c|c|}
\hline Método & $\delta t(\mathrm{~s})$ & \multicolumn{4}{|c|}{ Número de iterações } \\
\cline { 3 - 6 } & & $t=0.14 \mathrm{~s}$ & $t=0.22 \mathrm{~s}$ & $t=0.26 \mathrm{~s}$ & $t=0.34 \mathrm{~s}$ \\
\hline Explícito & $5.0 \times 10^{-6}$ & 28000 & 44000 & 52000 & 68000 \\
\hline P1-IR & $1.3 \times 10^{-4}$ & 1144 & 1783 & 2102 & 2739 \\
\hline P1-CN & $1.0 \times 10^{-4}$ & 1401 & 2201 & 2601 & 3401 \\
\hline P1-AB/CN & $1.1 \times 10^{-4}$ & 1400 & 2201 & 2601 & 3400 \\
\hline P2-IR & $1.35 \times 10^{-4}$ & 1143 & 1783 & 2101 & 2738 \\
\hline P2-CN & $1.0 \times 10^{-4}$ & 1401 & 2200 & 2601 & 3401 \\
\hline P2-AB/CN & $1.0 \times 10^{-4}$ & 1400 & 2200 & 2600 & 3400 \\
\hline
\end{tabular}


a)
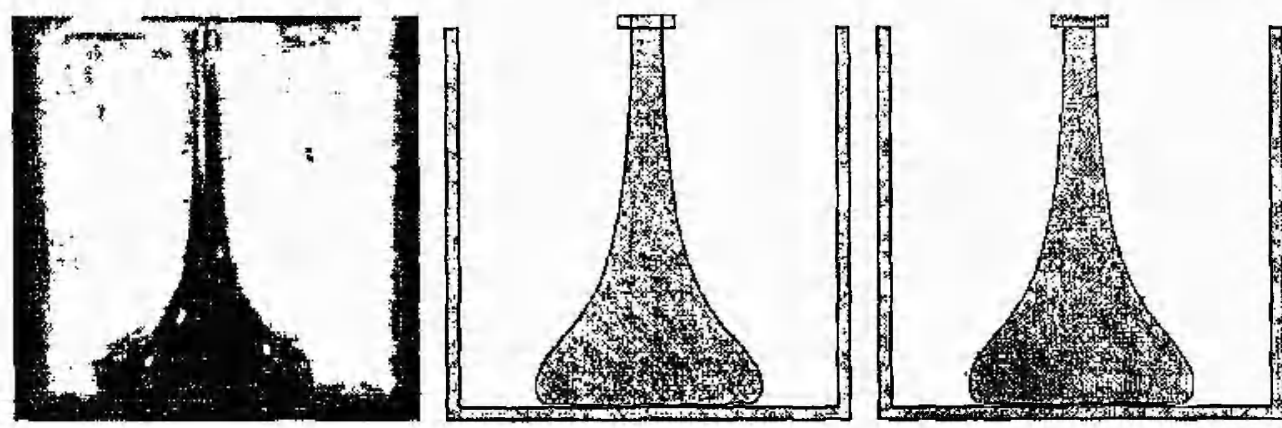

b)
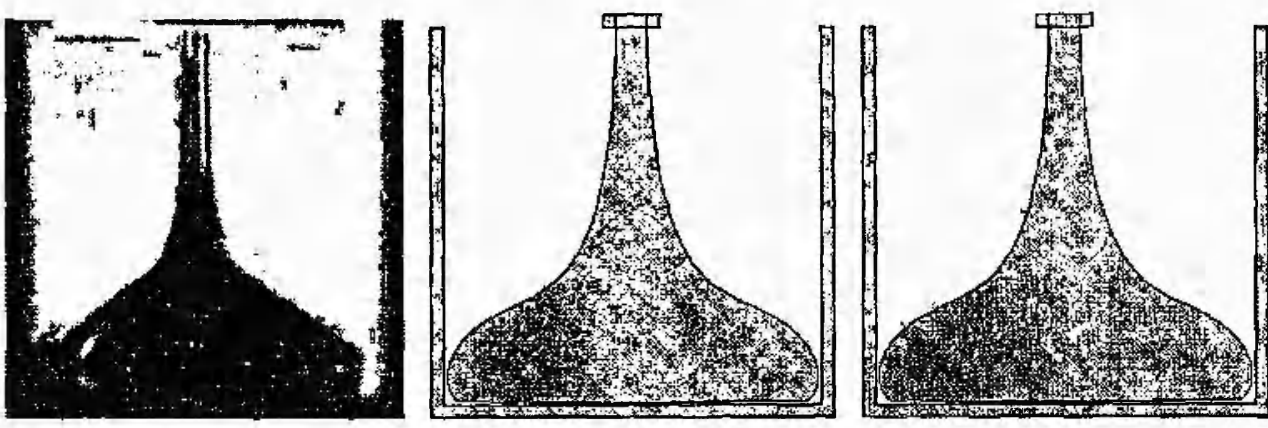

c)
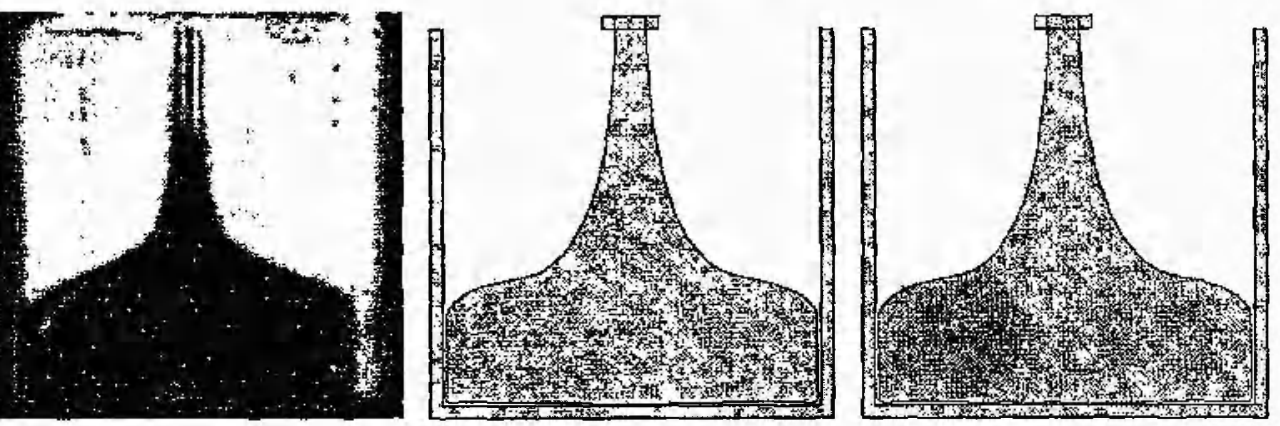

d)
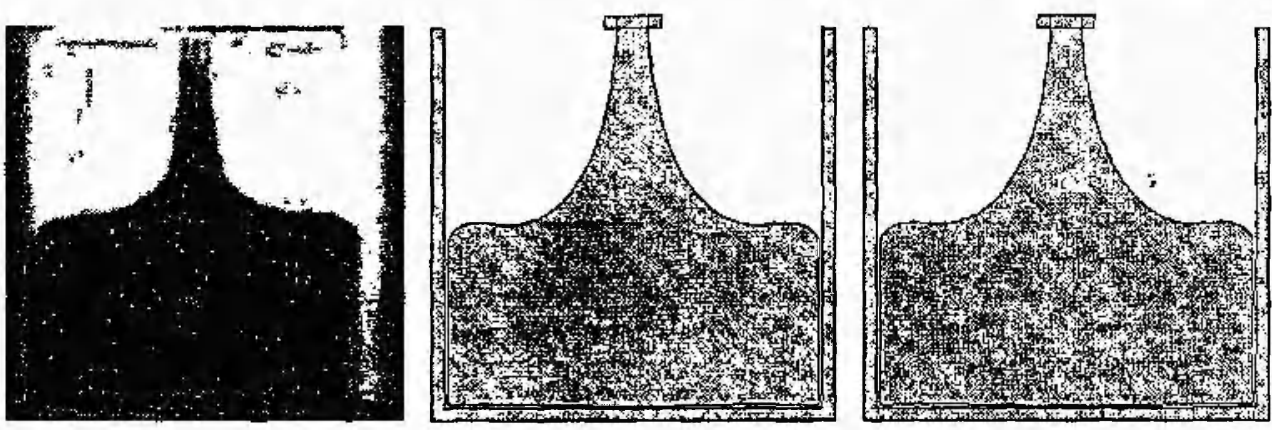

Figura 7.16: Comparação para o Modelo $1-R e=0.8$. Solução experimental (esquerda) e numérica (a solução do meio é obtida pelo método Projeção-2 utilizando a formulação IR, e a da direita, pelo método explícito). a) $t=0.14 \mathrm{~s}, \mathrm{~b}) t=0.22 \mathrm{~s}$, c) $t=0.26 \mathrm{~s} \mathrm{e} \mathrm{d}) t=0.34 \mathrm{~s}$. 
a)

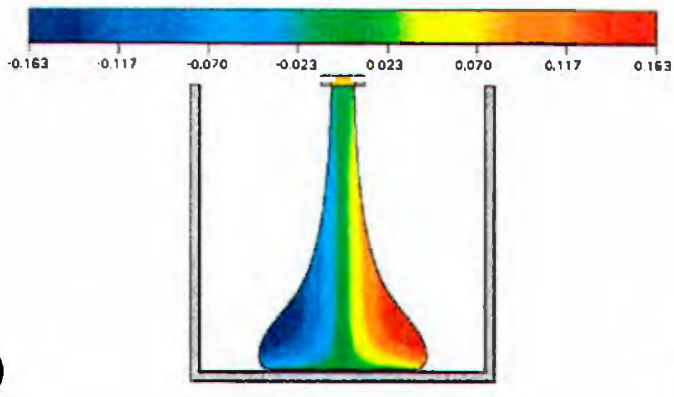

c)
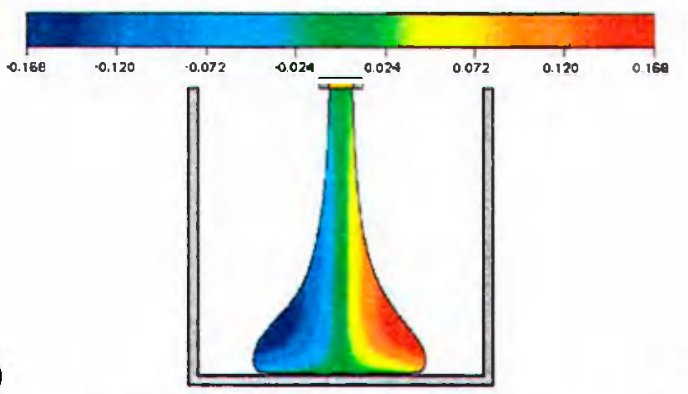

e)

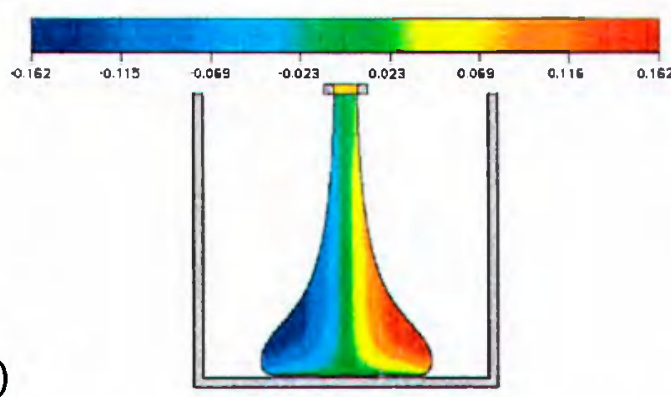

b)

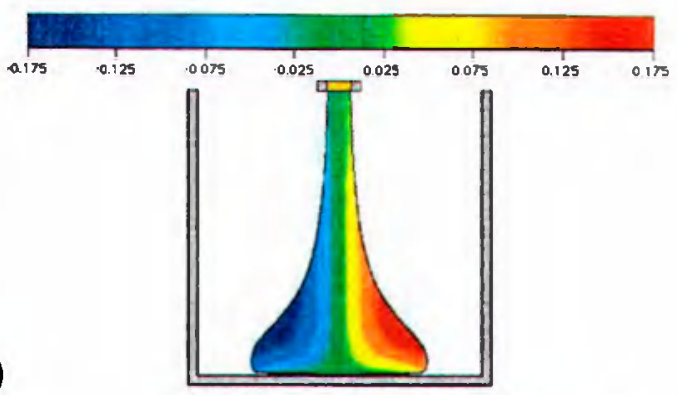

d)

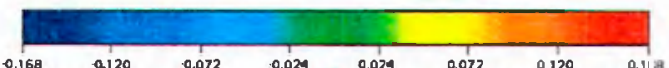

f)

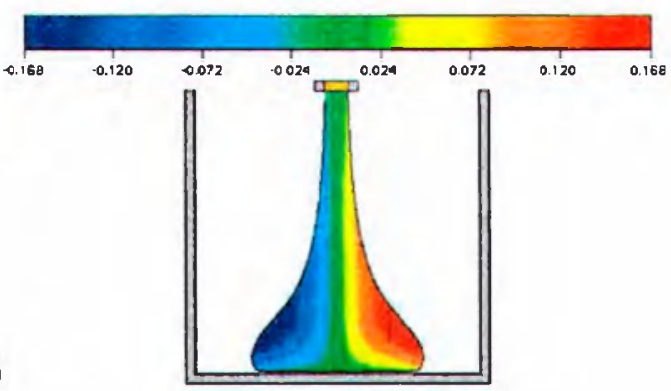

g)

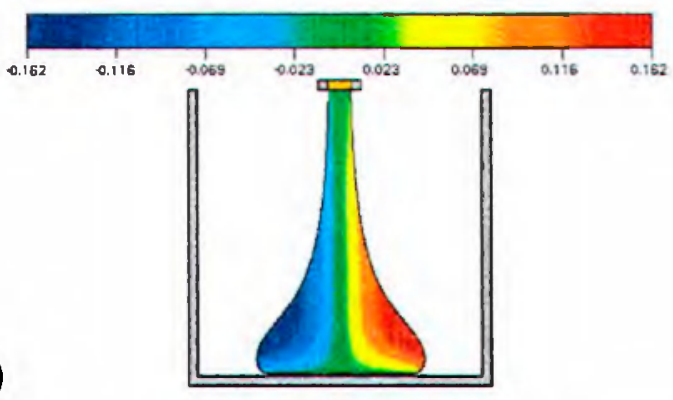

Figura 7.17: Comparação entre a velocidade $u$ para o Modelo $1-R e=0.8$, no tempo $t=0.14 \mathrm{~s}$. Métodos: a) Explícito, b)-d) Projeção-1 utilizando as formulaçōes IR, CN e $\mathrm{AB} / \mathrm{CN}$, respectivamente, e e)-g) Projeção-2 utilizando as formulações IR, CN e $\mathrm{AB} / \mathrm{CN}$. 
a)

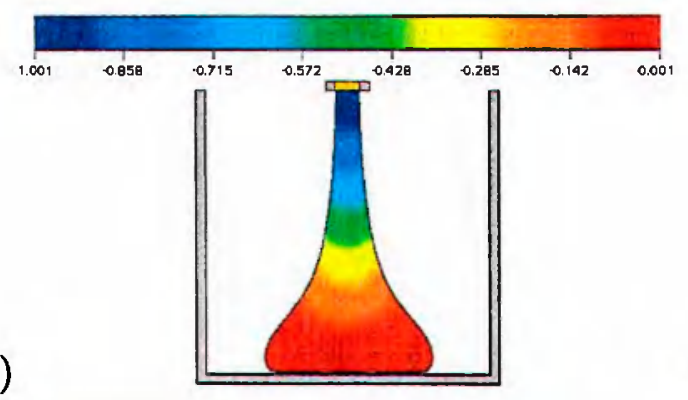

b)

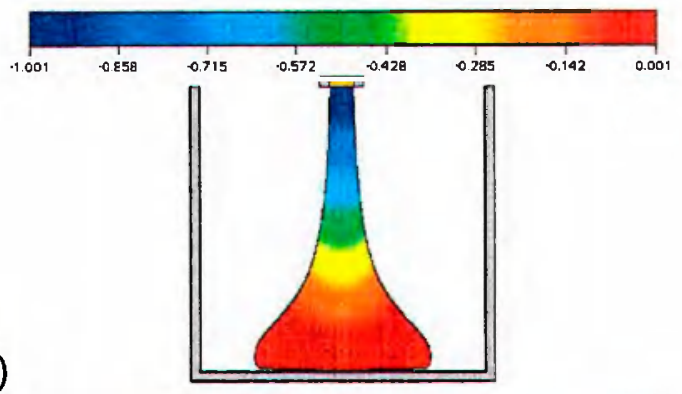

c)

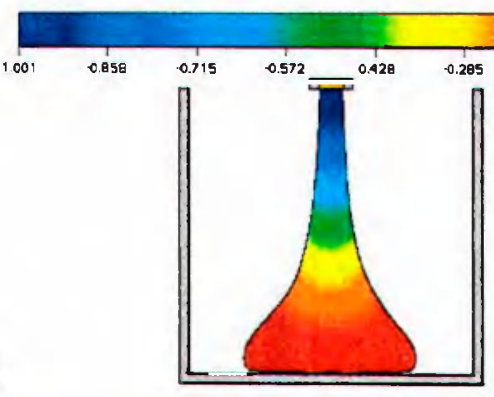

e)

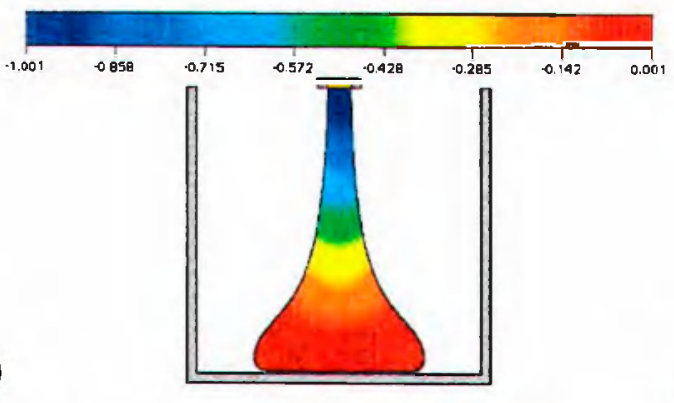

g)

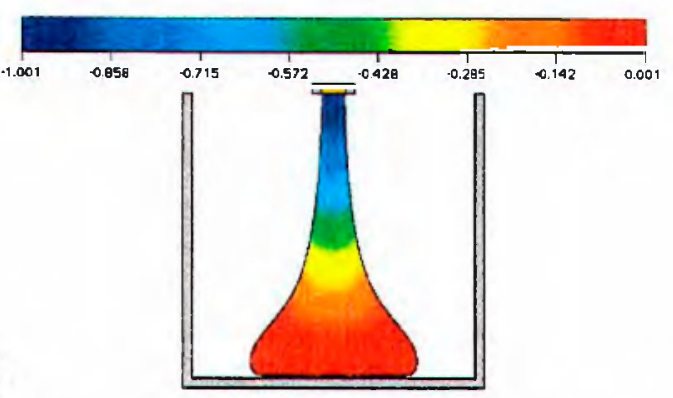

d)

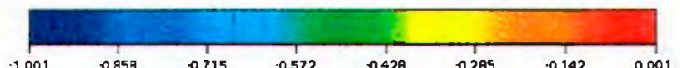

f)

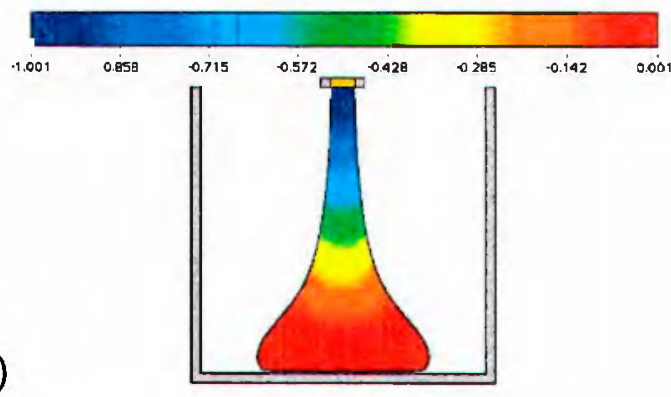

Figura 7.18: Comparação entre a velocidade $v$ para o Modelo $1-R e=0.8$, no tempo $t=0.14 \mathrm{~s}$. Métodos: a) Explícito, b)-d) Projeção-1 utilizando as formulações IR, CN e $\mathrm{AB} / \mathrm{CN}$, respectivamente, e e)-g) Projeção-2 utilizando as formulações IR, CN e $\mathrm{AB} / \mathrm{CN}$. 
Na segunda comparação qualitativa com os resultados experimentais de (Tomé et al., 1999) utilizou-se o mesmo domínio da figura 7.12, variando apenas o diâmetro do injetor e o número de Reynolds. Essas alterações produziram resultados distintos comparativamente com o experimento anterior, pelo fato de o número de Re ser um fator importante no comportamento do escoamento de um jato de fluido newtoniano. Para verificar as modificações ocorridas no escoamento e a concordância entre a solução experimental e as soluções numéricas foi aplicado o seguinte modelo:

Modelo 2- $R e=0.15$

- Dimensão do domínio: $0.05 \mathrm{~m} \times 0.05 \mathrm{~m}$;

- Diâmetro do injetor $(L): 0.003 \mathrm{~m}$;

- Velocidade de injeção $(U): 0.5 \mathrm{~ms}^{-1}$;

- Coeficiente de viscosidade cinemática $(\nu): 0.01 \mathrm{~m}^{2} \mathrm{~s}^{-\mathrm{i}}$;

- Parâmetros de escala: $L=0.003 \mathrm{~m} \mathrm{e} U=0.5 \mathrm{~ms}^{-1}$;

- Altura do injetor a partir da superfície rígida $(h)$ : $0.047 \mathrm{~m}$;

- Número de Reynolds $(R e=U L / \nu)$ : 0.15;

- Número de Froude $(F r=U / \sqrt{g} L): 2.9145$.

Esse modelo simula o escoamento de um jato sob o efeito da gravidade numa malha de $100 \times 100$ células computacionais $\left(\delta_{x}=\delta_{y}=0.00050 \mathrm{~m}\right)$, respeitando as restrições (7.17) descrita por (Cruickshank e Munson, 1981), ou seja, impondo o efeito fiambagem. $\mathrm{Na}$ figura 7.19, mostra-se a comparação entre os métodos explícito e Projeção-2 utilizando a formulação CN, e o resultado experimental de (Tomé et al., 1999). Nota-se que o resultados estão em boa concordância com o resultado experimental. Novamente, como os resultados obtidos pelo métodos Projeção- 1 utilizando as formulações IR, CN e $\mathrm{AB} / \mathrm{CN}$ e o método Projeção-2 utilizando as formulações IR e $\mathrm{AB} / \mathrm{CN}$ foram muito próximos daquele do método Projeção-2 utilizando a formulação $\mathrm{CN}$, apenas um desses resultados foi apresentado na figura 7.19. De acordo com a tabela 7.14, o valor de $\delta t$ permitido pelas formulações implícitas está entre 10 a 40 vezes maior do que aquele do método explícito, mostrando mais uma vez a eficiência dos métodos Projeção-1 e Projeção-2 utilizando as FI. Uma observação importante deve ser considerada a respeito 
da formação das flambagens para os métodos com formulações implícitas, em particular para o método Projeção- 2 utilizando a formulação IR. Com o crescimento do passo $\delta t$ retarda-se a ocorrência de flambagem, como observado na figura 7.20. Acredita-se que esse fato ocorre porque o efeito flambagem é uma solução estável para um problema que possui uma outra solução que não apresenta esse efeito. O método numérico inicia perseguindo esta última, pois ela é a única solução inicialmente, com valores de $\delta t$ muito grandes, e demora em "perceber" a existência da outra solução e passar a persegui-la por ela ser mais estável.

Tabela 7.14: Modelo 2 - Re $=0.15, L=0.004 \mathrm{~m} \mathrm{e} U=1.0 \mathrm{~ms}^{-1}$.

\begin{tabular}{|c|c|c|c|c|c|}
\hline Método & $\delta t(\mathrm{~s})$ & \multicolumn{4}{|c|}{ Número de iterações } \\
\cline { 3 - 6 } & & $t=0.37 \mathrm{~s}$ & $t=0.43 \mathrm{~s}$ & $t=0.47 \mathrm{~s}$ & $t=0.66 \mathrm{~s}$ \\
\hline Explícito & $2.5 \times 10^{-6}$ & 148000 & 172000 & 188000 & 264000 \\
\hline$P 1-\mathrm{IR}$ & $3.125 \times 10^{-5}$ & 11840 & 13760 & 15040 & 21120 \\
\hline$P 1-\mathrm{CN}$ & $2.5 \times 10^{-5}$ & 14800 & 17200 & 18800 & 26400 \\
\hline P1-AB/CN & $2.5 \times 10^{-5}$ & 14800 & 17200 & 18800 & 26400 \\
\hline P2-IR & $1.0 \times 10^{-4}$ & 3947 & 4587 & 5014 & 7040 \\
\hline P2-CN & $2.5 \times 10^{-5}$ & 14800 & 17200 & 18800 & 26400 \\
\hline P2-AB/CN & $2.5 \times 10^{-5}$ & 14800 & 17200 & 18800 & 26400 \\
\hline
\end{tabular}


a)
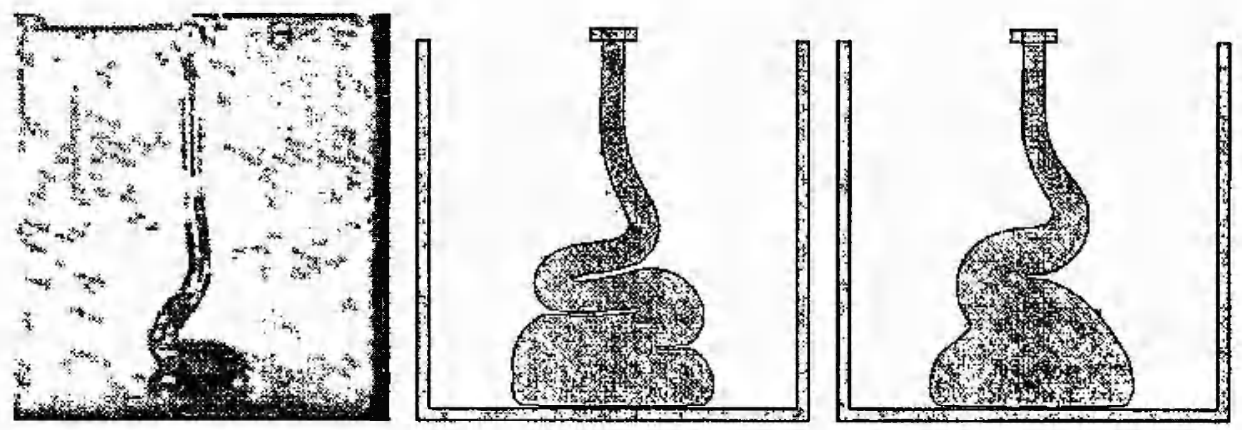

b)
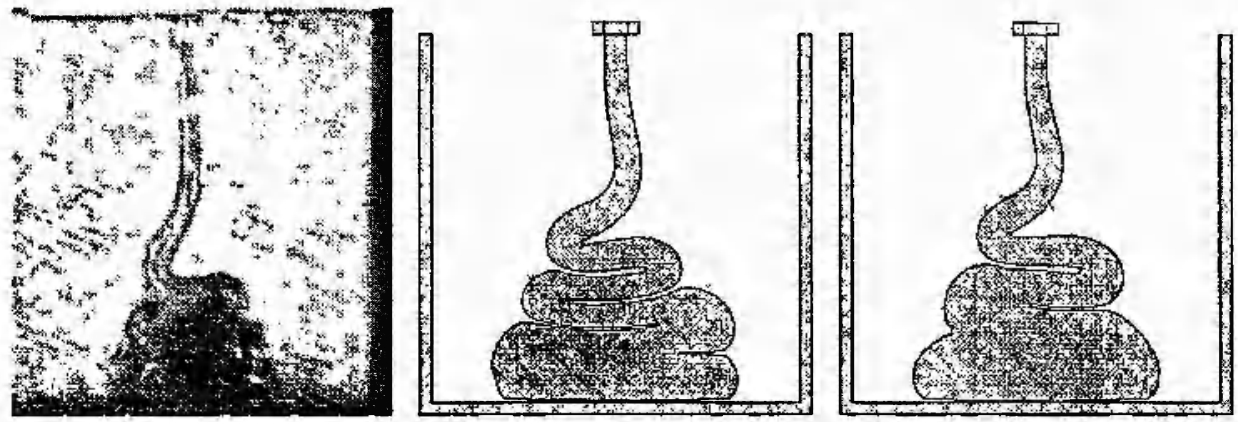

c)
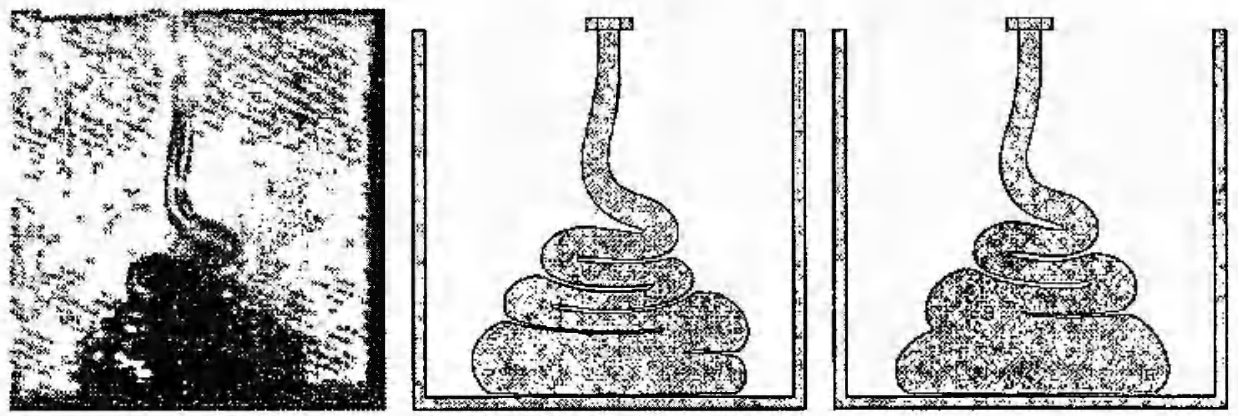

d)
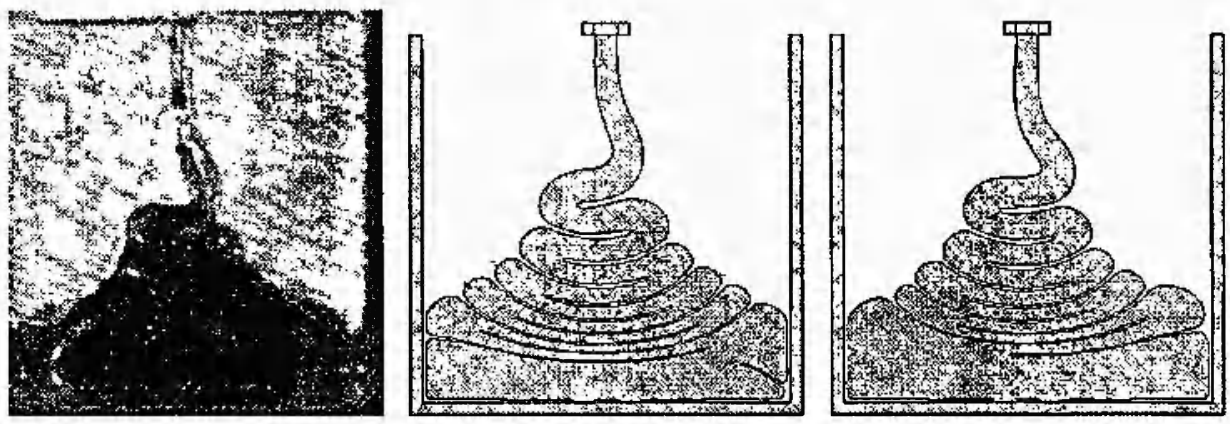

Figura 7.19: Comparação para o Modelo $2-R e=0.15$. Solução experimental (esquerda) e numérica (a solução do meio é obtida pelo método Projeção-2 utilizando a formulação $\mathrm{CN}$, e a da direita, pelo método explícito). a) $t=0.37 \mathrm{~s}, \mathrm{~b}) t=0.43 \mathrm{~s}$, c) $t=0.47 \mathrm{~s} \mathrm{e} \mathrm{d)} t=0.66 \mathrm{~s}$. 
a)

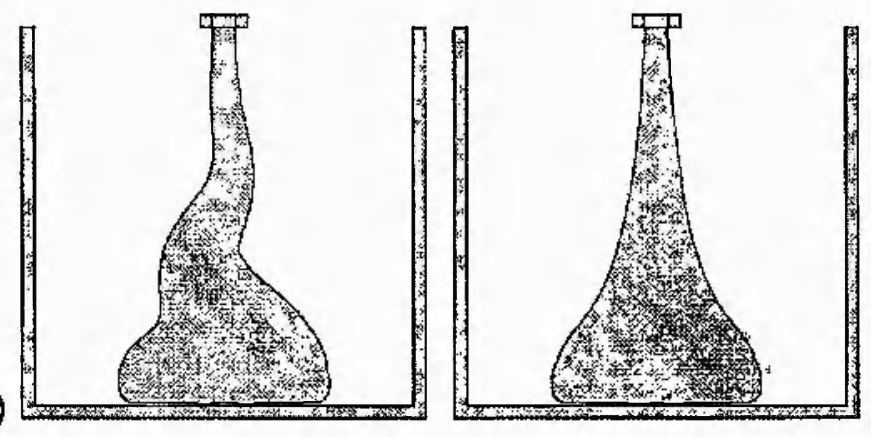

b)

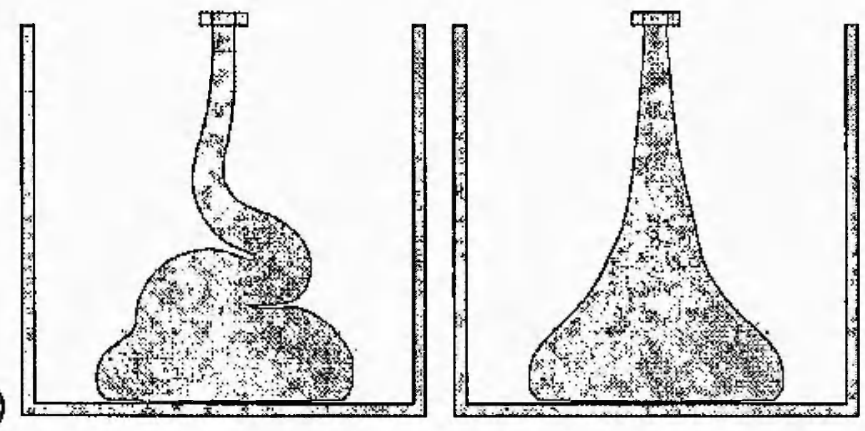

c)

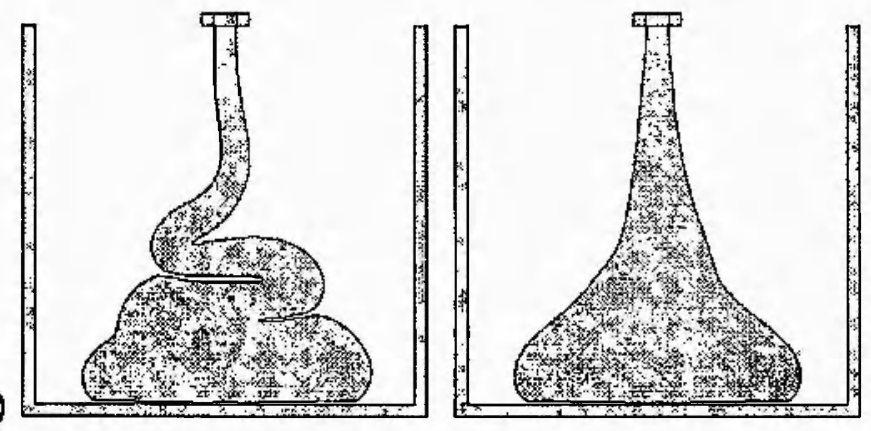

d)

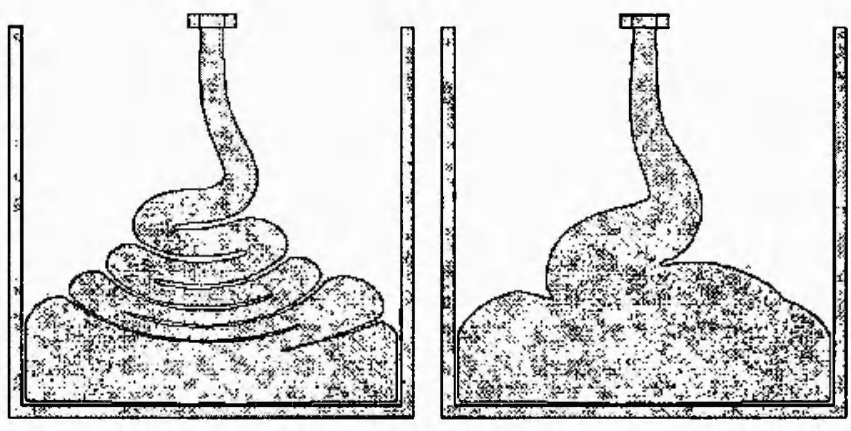

Figura 7.20: Comparação entre soluções numéricas obtidas pelo método Projeção-2 utilizando a formulação IR. A coluna esquerda mostra o desenvolvimento do método para $\delta t=6.25 \times 10^{-5} \mathrm{~s}$, e a coluna direita, para $\delta t=2.0 \times 10^{-4} \mathrm{~s}$. As soluções numéricas são obtidas nos tempos: a) $t=0.37 \mathrm{~s}, \mathrm{~b}) t=0.43 \mathrm{~s}, \mathrm{c}) t=0.47 \mathrm{~s}$ e d) $t=0.66 \mathrm{~s}$. 


$\left[\begin{array}{c}\text { CAPÍTULO } \\ 8\end{array}\right.$

\section{Conclusões e Futuros Trabalhos}

Os objetivos deste capítulo são os de sintetizar os resultados dos capítulos anteriores, apresentar algumas considerações finais dos resultados obtidos e a contribuição na área da DFC e apresentar uma proposta para trabalhos futuros.

\subsection{Sintese do Trabalho}

No capítulo 2 desta dissertação foram apresentadas as técnicas básicas de discretização de equações diferenciais parciais por diferenças finitas e suas formulações explícita e implícita. Descreveu-se os conceitos de consistência, estabilidade e convergência.

Com o objetivo de familiarizar o leitor com as equações de Navier-Stokes, o capítulo 3 apresentou as leis de conservação com uma breve análise do seu significado físico. Ainda no capítulo 3, discorreu-se sobre fluidos newtoniano, escoamentos viscosos, adimensionalização das equações de conservação e uma breve discussão de condições iniciais e de contorno.

A idéia geral da família de métodos de projeção e das formulações implícitas para as equações de Navier-Stokes foram apresentadas e discutidas no capítulo 4. Analisou-se o teorema DHH e sua aplicação no método de projeção, e também, as diversas maneiras de se aplicar formulações implícitas em Navier-Stokes. Neste capítulo, para o leitor interessado, há uma revisão bibliográfica minuciosa sobre os conceitos desenvolvidos. 
O capítulo 5 descreveu a técnica de solução numérica para escoamentos incompressíveis, o método GENSMAC. Discutiram-se o procedimento computacional, as condições de estabilidade e as condições iniciais e de contorno. Na segunda parte foram abordadas as formulações implícitas e variações do método de projeção na metodologia GENSMAC. Finalmente, na terceira parte, o método GBC foi analisado.

As equações aplicadas neste trabalho foram discretizadas no capítulo 6. Neste capítulo o ambiente de simulação FreeFlow2d foi apresentado juntamente com os módulos que o compõem.

E, finalmente, analisou-se no capítulo 7 algumas simulações numéricas utilizando as formulações implícitas introduzidas na metodologia GENSMAC para problemas bidimensionais. Apresentou-se a aplicação dessas formulações na simulação dos problemas: escoamento em um canal e em jatos. Foram realizadas validações com soluções analíticas e experimentais, e algumas comparações com o método explícito.

\subsection{Considerações Finais sobre os Resultados}

Os resultados e conclusões dos problemas de escoamentos de fluidos apresentados neste trabalho foram discutidos no capítulo 7. Entretanto, uma breve consideração final será apresentada.

As formulações implícitas apresentaram um desempenho satisfatório nos problemas transientes com superfície livre. As validações analítica e experimental comprovaram a capacidade dessas formulações de resolver problemas com baixo número de Reynolds $(\operatorname{Re}<<1)$.

Os métodos de Projeção-1 e Projeção-2 apresentaram diferentes resultados na simulação de um escoamento no canal. Como foi observado nas tabelas e figuras, o método Projeção-1 utilizando as formulações implícitas apresentou erros maiores que o métodos explícito e Projeção-2. Os resultados mostram que é necessário diminuir o passo temporal $\delta t$ no método Projeção-1 para que este apresente erros em um nível aceitável.

Nas simulações de jatos, os resultados numéricos obtidos pelos métodos Projeção-1 e Projeção-2 utilizando as formulações implícitas foram próximos entre si e daqueles do 
método explícito. Os resultados comprovaram a capacidade desses métodos de simularem problemas com superfícies livres.

O método Gradiente Bi-Conjugado Precondiconado foi utilizado pelas formulações implícitas na resolução do sistema linear resultante para obtenção do potencial $\psi$. Esse método demonstrou-se satisfatório, convergindo para a solução esperada em um número razoável de iterações.

Os resultados numéricos mostraram que as formulações implícitas superaram a condição de estabilidade do método explícito, e em muitos casos, a única restrição imposta para essas formulações foi a condição CFL. Entretanto, as formulações CN e $\mathrm{AB} / \mathrm{CN}$ introduziram oscilações, e como consequência, o valor de $\delta t$ permitido foi mais restrito do aquele da formulação IR. Embora as formulações $\mathrm{CN}$ e $\mathrm{AB} / \mathrm{CN}$ tenham permitido um passo temporal maior que o método explícito, a formulação IR mostrou ser mais estável permitindo valores para $\delta t$ bem maiores. Porém, recomenda-se escolher o valor do passo temporal com muito cuidado, para que a precisão numérica não seja afetada. Mesmo utilizando formulações implícitas, um estudo sobre $\delta t$ a ser utilizado deve ser feito com muita atenção.

Um dos objetivos principais deste trabalho foi a comparação das formulações implícita e explícita. Propriedades como o valor do passo temporal $\delta t$, a precisão numérica, o número de iterações e o tempo de processamento foram apresentadas em tabelas comparativas. Em todas as simulações, as formulações implícitas superaram o valor do passo temporal do método explícito, sendo que em alguns casos, o $\delta t$ foi aproximadamente 500000 vezes maior que o do método explícito. Os métodos Projeção-1 e Projeção-2 utilizando as formulações implícitas apresentaram erros próximos ao do método explícito com número de iterações bem menor. O tempo de processamento exigido pelas formulações implícitas foram significativamente menores que a formulação explícita.

Portanto, na opinião do autor, tudo indica que, tomando alguns cuidados, os métodos Projeção-1 e Projeção-2 utilizando as formulações implícitas são eficientes e capazes de resolver problemas viscosos com superfícies livres. Entre esses métodos, de acordo com os resultado numéricos, o método Projeção-2 utilizando a formulação IR mostrou ser o mais indicado. 


\subsection{Contribuição}

A maior contribuição desta dissertação é para o grupo de pesquisa em Matemática Aplicada do LCAD/ICMC-USP, pois este grupo está trabalhando no desenvolvimento de um sistema de simulação para escoamentos transientes incompressíveis baseado na técnica MAC (definido como FreeFlow), e as formulações implícitas serão muito utilizadas em problemas viscosos. Ou seja, uma dificuldade no ambiente FreeFlow2D, devido à discretização temporal explícita é na simulação de problemas com baixo número de Reynolds. Com a implementação das formulações implícitas na metodologia GENSMAC, resultados satisfatórios foram apresentados e novas idéias surgiram, motivando o estudo de métodos implícitos para a resolução das equações de Navier-Stokes.

Portanto, a presente dissertação contribui no campo de DFC, na resolução numérica das equações de Navier-Stokes em escoamentos com superfícies livres utilizando formulações implícitas na metodologia GENSMAC.

Os principais resultados numéricos obtidos neste trabalho serão apresentados à comunidade de DFC em artigos. Alguns desses resultados foram comunicados em (Oishi et al., 2003), e em resumos submetidos ao III CONGRESSO NACIONAL DE ENGENHARIA MECÂNICA - CONEM2004,X ENCONTRO NACIONAL DE CIÊNCIAS TÉRMICAS - ENCIT2004 entre outros, e à revista Tendências em Matemática Aplicada e Computacional - TEMA2003.

\subsection{Futuros Trabalhos}

Os aspectos mais relevantes dos temas que serão abordados em futuros trabalhos são :

- Estudo de métodos de projeção de ordem mais alta (Strikwerda e Lee, 1999), (Brown et al., 2001), (Chang et al., 2002), (Denaro, 2003) e de condições de contorno apropriadas (Chorin, 1968), (Kan, 1986), (Gresho, 1990), (Weinan e Liu, 1995) e (Lee et al., 2001).

- Estudo de estabilidade como aqueles em (Petersson, 2001) para os métodos implícitos no contexto das equações de Navier-Stokes;

- Estudo de outros métodos iterativos (Saad e Schltz, 1986), (Ayachour, 2003);

- Extensão deste trabalho para o caso tridimensional. 


\section{Referências Bibliográficas}

Ames, W. F. (1972). Nonlinear partial differential equations in engineering. Vol II. Academic Press, New York.

Amsden, A. e Harlow, F. H. (1970). A simplified mac technique for incompressible fluid flow calculations. Journal of Computational Physics, 6:332-335.

Anderson, J. D. (1995). Computational Fluid Dynamics. McGraw-Hill - N.Y.

Aris, R. (1962). Vectors, tensors, and the basic equation of fluid mechanics. Dover.

Armenio, V. (1997). An improved mac method (simac) for unsteady high-reynolds free surface flows. International Journal for Numerical Methods in Fluids, 24:185-214.

Ayachour, E. (2003). A fast implementation for gmres method. Journal of Computational and Applied Mathematics, 159:269-283.

Bansch, E. (1998). Simulation of instationary, incompressible flows. Acta Math. Univ. Comenianae, LXVII:101-114.

Batchelor, G. K. (1970). An Introduction to Fluid Dynamics. Cambridge.

Beam, R. M. e Warming, R. F. (1982). Implicit numerical methods for the compressible Navier-Stokes and Euler equations. Von Karman Institute Lectures Serie, Rhode Saint Genese, Belgium.

Bell, J. B., Colella, P., e Glaz, H. M. (1989). A second order projection method for the incompressible Navier-Stokes equations. Journal of Computational Physics, $85: 257-283$.

Bell, J. B., Colella, P., e Howell, L. H. .(1991). An efficient second-order projection method for viscous incompressible flow. Proceedings of the Tenth AIAA Computational Fluid Dynamics Comference, AIA A, June:360. 
Bentley, J. (1986). Programming Pearls. Addison-Wesley, Reading, MA.

Bijl, H., Ampenter, M. H., Vatsa, V., e Kennedy, C. (2002). Implicit time integration schems for the unsteady compressible Navier-Stokes equations: laminar flows. Journal of Computational Physics, 179:313-329.

Botella, O. (1997). On the solution of the Navier-Stokes equations using chebyshev projection schems with third-order accuracy in time. Computers \& Fluids, 26:107116.

Bretas, R. E. S. e D'Avila, M. A. (2000). Reologia de Polímeros Fundidos. Editora da UFScar.

Briggs, W. L., Newell, A. C., e Sarie, T. (1983). Focusing: a mechanism for instability of nonlinear finite difference equations. Journal of Computational Physics, 51:83-106.

Briley, W. (1971). A numerical study of laminar separation bubbles using the NavierStokes equations. Journal Fluid Mech., 47:713-736.

Briley, W. e McDonald, H. (1975). Solution of the three-dimensional compressible Navier-Stokes equation by an implicit technique. Proceedings of the Fourth International Conference on Numerical Methods in Fluid Dynamics. New York.

Briley, W. e McDonald, H. (1977). Solution of the multi-dimensional compressible Navier-Stokes equations by a generalized implicit method. Journal of Computational Physics, 24:372-397.

Briley, W. R. e McDonald, H. (2001). An overview and generalization of implicit NavierStokes algorithms and approximate factorization. Computers \& Fluid, 30:807-828.

Brown, D. L., Cortez, R., e Minion, M. L. (2001). Accurate projection methods for the incompressible Navier-Stokes equations. Journal of Computational Physics, 168:464-499.

Caretto, L. S., Gosman, A. D., e Spalding, S. V. P. D. B. (1972). Two calculation procedures for steady, three dimensional flows with recicurlation. Lectures Notes in Physics, 19.

Castelo, A., Cuminato, J., Oliveira, M., Santri, R., Tomé, M., e César, C. (1996). FreeFlow3D: Um sistema integrado para simulaçäo de escoamentos incompressiveis com superficies livres. Relatório Técnico, ICMC-USP-São Carlos, Brasil. 
Castelo, A., Tomé, M., Cuminato, J., Oliveira, J., Mangiavacchi, N., Fortuna, A., e McKee, S. (1999). Surface tension implemention for gensmac2d. XV Congresso Brasileiro de Engenharia Mecônica-COBEM.

Casulli, V. (1981). Numerical simulation of free-surface thermally influenced flows for nonhomogeneous fluids. Applied Mathematics and Computation, 8:261-280.

Casulli, V. (1990). Semi-implicit finite difference methods for the two-dimensional shallow water equations. Journal of Computational Physics, 86:56-74.

Chang, W., Giraldo, F., e Perot, B. (2002). Analysis of an exact fractional step method. Journal of Computational Physics, 180:183-199.

Chapra, S. C. e Canale, R. P. (1990). Numerical Methods for Enginears, 2nd ed. McGraw-Hill International Editions, Applied Mathematics Series.

Charney, J. G., Fjortoft, R., e Neumann, J. V. (1950). Numerical integration of the barotropic vorticity equation. Tellus, 2:237-254.

Chattopadhyay, H. e Saha, S. K. (2003). Turbulent flow and heat transfer from a slot jet impinging on a moving plate. International Journal Heat and Fluid Flow, 24:685-697.

Chorin, A. (1967). A numerical method for solving incompressible viscous flow problems. Journal of Computational Physics, 2:12-26.

Chorin, A. (1968). Numerical solution of the Navier-Stokes. Mathematics of Computation, 2:745-762.

Courant, R., Friedrichs, K., e Lewy, H. (1967). On the partial differential equations of mathematical physics. IBM Journal Research and Development, 11:215-234.

Courant, R. e Hilbert, D. (1962). Methods of Mathematical Physics, V.2. Wiley, New York.

Courant, R., Issacson, E., e Rees, M. (1952). On the solution of nonlinear hyperbolic differential equations by finite differences. Commun. Pure Appl. Math., 5:243-255.

Crank, J. e Nicolson, P. (1947). A practical method for numerical evaluation of solution of partial differential equations of the heat-conduction type. Proc. Camb. Phi. Soc., 43:50-67. 
Cruickshank, J. O. e Munson, B. R. (1981). Viscous-fluid buckling of plane and axisymmetric jets. Journal of Fluid Mech., 113:221-239.

Cuminato, J. A. e Meneguette, M. (1999). Discretização de Equações Diferenciais Parciais: Técnica de Diferenças Finitas. Em preparação.

Cunha, M. C. C. (2000). Métodos Numéricos. Editora Unicamp, Campinas.

Dartzi, P., Yang, Y., e Chang, C. (1998). Computation of internal flow with free surface using artificial compressibility. Numer. Heat Transfer B, 33:119-134.

Denaro, F. M. (2003). On the applications of the helmoltz-hodge decomposition in projection methods for incompressible flows with general boundary conditions. International Journal for Numerical Methods in Fluids, 43:43-69.

Dennis, C., Ingham, D., e Cook, R. (1979). Finite-difference methods for calculating flows in three dimensions. Journal of Computational Physics, 33:325-339.

Deville, M. O. (1975). An alterning direction implicit algorithm for viscous free surface flows. J. de Mécanique, 14:161-187.

Doricio, J. L. (2003). GENSMAC-SOF: Um método numérico para simular escoamentos incompressiveis de fluido de segunda ordem. Dissertação de Mestrado, Biblioteca Digital de Teses e Dissertações-www.teses.usp.br, ICMC-USP-São Carlos, Brasil.

Douglas, J. e Kim, S. (2001). Improved accuracy for locally one-dimensional methods for parabolic equations. Mathematical Models and Methods in Applied Sciences, 11:1563-1579.

Douglas, J. e Rachford, H. (1960). On the numerical solution of heat conduction problems in two and three space variables. Transactions of the American Mathematical Society, 82:421-439.

Eddy, E. P. (1949). Stability in the Numerical Solution of Initial Value Problems in Partial Differential Equations. NOLM, Naval Ordnace Laboratory, White Oak, Silver Springer, Maryland.

Ferreira, V., Tomé, M., Castelo, A., Cuminato, J., Mangiavacchi, N., e McKee, S. (2002). High order upwinding and the hydraulic jump. International Journal of Numerical Methods in Fluids, 39:549-583. 
Ferreira, V. G. (2001). Análise e implementação de esquemas de conveç̧ão e modelos de turbulência para simulação de escoamentos incompressiveis envolvendo superficies livres. Tese de Doutorado, Biblioteca Digital de Teses e Dissertaçõeswww.teses.usp.br, ICMC-USP-São Carlos, Brasil.

Ferziger, J. H. e Períc, M. (1999). Computational Methods for Fluid Dynamics. 2. Heidelberg, Springer-Verlag.

Flecther, C. A. (1992). Computational Techniques for Fluid Flous. Heidelberg, Springer-Verlag.

Fletcher, R. (1975). Numerical Analysis Dundee. Springer-Verlag- Lectures Notes in Mathematics, vol.506.

Fortuna, A. O. (2000). Técnicas Computacional para Dinâmica dos Fluidos. Edusp.

Freund, R. e Nachtigal. N. (1991). Qmr: a quasi-minimal residual method for nonhermitian linear system. Numer. Math, 60:315-339.

Fromm, J. E. (1968). A method for reducing dispercion in convective difference schemes. Journal of Computional Physics, 3:217-223.

Gaskell, P. e Lau. A. (1988). Curvature-compensated convective transport: Smart, a new boundedness-preserving transport algorithm. International Journal for $\mathrm{Nu}$ merical Methods in Fluids, 8:617-641.

Golub, G. H. e Loan, C. F. V. (1989). Matrix Computations, 2nd e. Baltimore: Jonhs Hopkins Universaty Press.

Gresho, P. M. (1990). On the theory of semi-implicit projection methods for viscous incompressible flow and its implemention via a finite element method that also introduces a nearly consistent mass matrix. International Journal for Numerical Melhods in Fluids, 11:587-620.

Gresho. P. M. e Chan, S. T. (1990). On the theory of semi-implicit projection methods for viscous incompressible flow and its implemention via a finite element method that also introduces a nearly consistent mass matrix. part 2: Implementation. International Journal for Numerical Methods in Fluids, 11:621-659. 
Gresho, P. M. e Sani, R. (1987). On pressure on boundary conditions for the incompressible Navier-Stokes equations. International Journal for Numerical Methods in Fluids, 7:1111-1145.

Griebel, M., Dornseifer, T., e Neunhoeffer, T. (1998). Numerical Simulation in Fluid Dynamics: A Practical Introduction. Society for Industrial and Applied Mathematics - SIAM.

Grossi, L. (2003). Desenvolvimento de métodos numéricos para a simulação de escoamentos não-newtonianos e viscoelástico com superfície livre. Tese de Doutorado, Biblioteca Digital de Teses e Dissertações-www.teses.usp.br, ICMC-USP-São Carlos, Brasil.

Hackbush, W. (1994). Iterative Solution of Large Sparse System of Equations. SpringerVerlag, New York.

Hairer, E. e Wanner, G. (1991). Solving Ordinary Differential Equations II: Stiff and Differential - Algebrics Problems. Springer-Verlag.

Harlow, F. e Welch, J. (1965). Numerical calculation of time-dependent viscous incompressible flow of fluid with free surface. Physics of Fluids, 8:2182-2189.

Henshaw, W. D. (1994). A fourth-order accurate method for the incompressible NavierStokes equations on overlapping grids. Journal of Computational Physics, 13:113.

Heywwod, J. e Rannacher, R. (1986). Finite element approximation of the nonstationary Navier-Stokes, part ii; stability of solution and error estimates uniform in time. Society for Industrial and Applied Mathematics Journal of Numerical and Analitical, 23:750-777.

Hirsch, C. (1989). Numerical Computational of Internal and External Flows, volume 1. A Wiley-Interscience Publication.

Hirt, C., Amsden, A., e Cook, J. (1974). An arbitrary lagrangian-eulerian computing method for the all flow speeds. Journal of Computational Physics, 14:227-253.

Hirt, C. e Cook, J. (1972). Calculating three-dimensional flows around structure and over rough terrain. Journal of Computational Physics, 10:324-340.

Hirt, C. e Nichols, B. (1971). Improved free surface boundary-conditions for numerical incompressible-flow calculations. Journal of Computational Physics, 8:434-448. 
Hirt, C. e Nichols, B. (1981). Volume of fluid (vof) method for the dynamics of free boundaries. Journal of Computational Physics, 39:201-225.

Hirt, C. W. (1968). Heuristic stability theory for finite-difference equations. Journal of Computational Physics, 2:339-355.

Hodge, W. V. D. (1952). The Theory and Applications of Harmonic Integrals. Cambridge University Press, Cambridge.

Hoffmann, K. A. e Chiang, S. T. (1993). Computational Fluid Dynamics for Engineers, volume 1. A Publication of Engineering Eduaction System, USA.

Hugues, S. e Randriamampianina, A. (1998). An improved projection scheme applied to pseudospectral methods for the incompressible Navier-Stokes equations. International Journal for Numerical Methods in Fluids, 28:501-521.

Johnston, H. e Liu, J. G. (2002). Finite difference schemes for incompressible flow based on local pressure boundary conditions. Journal of Computational Physics, 180:120-154.

Kan, J. V. (1986). A second-order accurate pressure-correction schemes for viscous incompressible flow. SIAM Journal on Scientific Computing, 7:870-891.

Kim, J. e Moin, P. (1985). Application of a fractional-step method to incompressible Navier-Stokes equations. Journal of Computational Physics, 59:308-323.

Kim, K., Baek, S., e Sung, H. J. (2002). An implicit velocit decoupling procedure for the incompressible Navier-Stokes equations. International Journal for Numerical Methods in Fluids, 38:125-138.

Kincaid, D., Respess, J., Young, D., e Grimes, R. (1982). -. ACM Transaction on Mathematical Software, 8:302-322.

Knuth, D. (1968). Fundamental Algorithms - The Art of Computer Programming. Addison-Wesley, Reading, MA.

Ladyzhenskaja, O. A. (1963). The mathematical theory of viscous incompressible flow. Gordon and Breach, New York.

Lambert, J. D. (1973). Computational Methods in Ordinary Differential Equations. A Wiley Series. 
Lambert, J. D. (1991). Numerical Methods for Ordinary Differential Systems. A Wiley Series.

Lanczos, C. (1952). Solution of systems of linear equations by minimized iteration. Journal Res. Nat. Bur. Standards, 49:33-53.

Langtangen, H. P. e Winther, K. A. M. R. (2002). Numerical methods for incompressible viscous flow. Accepted for publication in Advances in Water Resources, Elsevier.

Lapidus, L. e Pinder, G. F. (1982). Numerical Solution of Partial Differential Equations in Science and Engineering. A Wiley-Interscience Publication, New York.

Larsson, S. (2002). Preservation of strong stability associated with analytic semigroups. Society for Industrial and Applied Mathematics Journal of Numerical and Analitical, pages 11-24.

Lax, P. D. e Richtmyer, R. D. (1956). Survey of the stability of linera finite differnce equations. Communications on Pure and Applied Mathematics, 17:267-293.

Lee, M., Oh, D., e Kim, Y. (2001). Canonical fractional-step methods and consisten boundary conditions for the incompressible naveir-stokes equations. Journal of Computational Physics, 168:73-100.

Leonard, B. P. (1979). A stable and accurate convective modelling procedure based on quadratic upstream interpolation. Comput. Meth. Appl. Mech. Engrg., 19:59-98.

LeVeque, R. (1990). Numerical Methods for Conservation Laws. Birkhauser-Verlag.

Liotta, S. F., Romano, V., e Russo, G. (2000). Central schemes for balance lows of relaxation type. Society for Industrial and Applied mathematics Journal of Numerical and Analitical, 38:1337-1356.

Luenberger, D. (1984). Linear and nonlinear programming. Addison-Wiley.

Maliska, C. A. (1995). Transferência de Calor e Mecânica dos Fluidos Computacional. LTC Editora - Rio de Janeiro.

Maliska, C. R. (1981). A solution method for three-dimensional parabolic fluid flow problems nonorthogonal coordinates. $\mathrm{PhD}$ thesis, Universty of Waterloo, Canadá. 
Manna, M. e Vacca, A. (1999). An efficient method for the solution of the incompressible Navier-Stokes equations in cylindrical geometries. Journal of Computational Physics, 151:563-584.

Mansour, M. L. e Hamed, A. (1990). Implicit solution of the incompressible NavierStokes equations on a non-staggered grid. Journal of Computational Physics, 86:147-167.

Mcdonough, J. M. e Bywater, R. J. (1986). Large-scale effects on local small-scale chaotic solutions to burger's equation. American Institute of Aeronautics and Astronautic Journal, 24:1929-1930.

Minion, M. (1994). Two methods for the study vortex pacth evolution on locally refined grid. $\mathrm{PhD}$ thesis, University of California, Berkeley, USA.

Minion, M. (1996). A projection method for loclly refined grids. Journal of Computational Physics, 127:158-177.

Miyata, H. e Masuko, A. (1985). Finite-difference simulation of nonlinear waves generated by ships of arbitrary three-dimensional configuration. Journal of Computational Physics, 60:391-436.

Oishi, C., Ferreira, V., Tomé, M., Castelo, A., Cuminato, J., e Mangiavacchi, N. (2003). Estudo e implementação de um método implícito no ambiente freeflow2d. XXVI Congresso Nacional de Matemática Aplicada e Computacional, São josé do Rio Preto, Brasil.

Oliveira, J. (1999). Desenvolvimento de um Sistema de Simulação de Escoamentos de Fluidos com Superfícies livres Bidimensionais. Dissertação de Mestrado, Biblioteca, ICMC-USP-São Carlos, Brasil.

Oliveira, J. (2000). Manual do FreeFlow2D. Relatório Técnico, ICMC-USP-São Carlos, Brasil.

Paige, C. e Saunders, M. A. (1975). Solution of sparse indefinite systems of linear equations. SIAM Journal Numer. Anal., 12:617-629.

Panton, R. L. (1984). Incompressible flow. John Wiley e Sons.

Patankar, S. (1980). Numerical Heat Transfer and Fluid Flows. Hemisphere Publishing Co. 
Patankar, S. V. e Spalding, D. B. (1972). A calculation procedure for heat, mass and momentum tranfer in three-dimensional parabolic flows. Int. Journal of Heat and Mass Transfer, 15:1787-1806.

Peaceman, D. e Rachford, H. (1955). The numerical solution of parabolic and elliptic diferential equations. Journal Society Industrial and Applied Mathematics, 3:28-41.

Pearson, C. (1965). A computation method for viscous flow problems. Journal Fluid Mech., 21:611-.

Perot, J. B. (1993). An analysis of the fractional step method. Journal of Computational Physics, 108:51-58.

Petersson, N. A. (2001). Stability of pressure boundary conditions for stokes and NavierStokes equations. Journal of Computational Physics, 172:40-70.

Peyret, R. e Taylor, T. D. (1983). Computational Methods for Fluid Flow. SpringerVerlag.

Pracht, W. E. (1971). A numerical method for calculating transient creep flows. Journal of Computational Physics, 7:46-60.

Press, W. H., Flannery, B. P., Teukolsky, S. A., e Vetterling, W. T. (1992). Numerical Recipes in $C$ : The Art of Scientific Computing, 2nd ed. Cambridge - University Press.

Price, H. S., Varga, R., e Warren, J. (1966). Applications of oscillation matrices to diffusion-correction equations. Journal Math. Physics, 45:301-311.

Quarteroni, A., Sacco, R., e Saleri, F. (2000). Numerical Mathematics. Springer.

Raithby, G. (1976). A critical evaluation of upstream differencing applied to problems involving fluid flow. Comput. Meth. Appl. Mech. Engrg., 9:75-86.

Ramaswamy, B. e Kawahara, M. (1987). Lagrangian finite element analysis applied to viscous free surface fluid flow. International Journal for Numerical Methods in Fluids, 7:953-984.

Richtmyer, R. D. e Morton, K. W. (1967). Difference Methods for Initial Value Problems. A John Wiley-Interscience Publication. 
Roache, P. J. (1998). Fundamental of Computational Fluid Dynamics. Albuquerque, Hermosa Publishers.

Saad, Y. (1993). A flexible inner-outer preconditioned gmres algorithm. SIAM J. Sci. Comput., 14:461-469.

Saad, Y. e Schltz, M. H. (1986). Gmres: A generalized minimal residual algorithm for solving nonsymmetric linear systems. SIAM Journal of Sci. Stat. Comput., $7: 856-869$.

Sabatini, M. H. (2002). Expansão do sistema FREEFLOW3D para escoamentos com influência a temperatura. Dissertação de Mestrado, Biblioteca Digital de Teses e Dissertações-www.teses.usp.br, ICMC-USP-São Carlos, Brasil.

Sadok, H. (1999). Cmrh: a new method for solving nonsymmetric linear system based on the hessemberg reduction algorithm. Numer. Algorithm, 20:303-321.

Siemieniuch, J. e Gladwell, I. (1978). Analysis of explicit difference methods for the diffusion-convection equation. International Journal of Numerical Methods in Engineering, 12:899-916.

Silva, G. F. (2003). Simulação numérica de escoamentos viscoelástico com superfície livre usando o ambiente FREEFLOWथD. Dissertação de Mestrado, Biblioteca Digital de Teses e Dissertações-www.teses.usp.br, ICMC-USP-São Carlos, Brasil.

Sod, G. A. (1989). Numerical Methods in Fluid Dynamics-Initial and Initial BoundaryValue Problems. Cambridge, New York.

Souza, F. S. (2002). Simulação numérica de escoamentos multifásicos usando o ambiente FREEFLOW3D. Dissertação de Mestrado, Biblioteca Digital de Teses e Dissertações-www.teses.usp.br, ICMC-USP-São Carlos, Brasil.

Spalding, D. (1972). A novel finite differential expression involving both first and second derivates. Int. Journal Numerical Meths Engrg., 4:551-563.

Street, S. A. R. (2003). The pressure accuracy of fractional-step methods for the NavierStokes equations on staggered grids. ANZIAM. Australian and New Zealand Industrial and Applied Mathematics, 44:20-39. 
Strikwerda, J. C. e Lee, Y. (1999). The accuracy of the fractional step method. Society for Industrial and Applied Mathematics Journal of Numerical and Analitical, 37:3747.

Thoman, D. e Szewczyk, A. A. (1966). Two dimensional flow of a viscous, incompressible fluid over stationary and rotating cylinders. Tech. Report. - Heat Transfear and Fluid Mechanics Lab., Dept. of Mechanical Engineering, University of Notre Dame., 66:14.

Thomas, J. W. (1995). Numerical Partial Differential Equations. Springer.

Tomé, M., Castelo, A., Murakami, J., Cuminato, J., Minghim, R., Oliveira, C., Mangiavacchi, N., e McKee, S. (2000). Numerical simulation of axisymmetric free surface flows. Journal of Computational Physics, 157:441-472.

Tomé, M., McKee, S., Barratt, L., Jarvis, D., e Patrick, A. (1999). An experimental and numerical investigations of container flling with viscous liquids. International Journal for Numerical Methods in Fluids, 31:1333-1353.

Tomé, M. F. e McKee, S. (1994). Gensmac: A computational marker-and-cell method for free surface flows in general domains. Journal of Computational Physics, 110:171-186.

Turek, S. (1996). A comparative study of some time-stepping techniques for the incompressible Navier-Stokes equations: From fully implicit nonlinear schemes to semi-implicit projection methods. International Journal for Numerical Methods in Fluids, 22:987-1011.

Vanka, S. P. (1986). Block-implicit multigrid solution of Navier-Stokes equations in primitive variable. Journal of Computational Physics, 65:138-158.

Varonos, A. e Bergeles, G. (1998). Development and assessment of a variable-order nonoscillatory for convection term discretization. International Journal for Numerical Methods in Fluids, 26:1-16.

Verwer, J. G. e Dekker, K. (1984). Stability of Runge-Kutta Methods for Stiff Nonlinear Differential Equations. North-Holland.

Viecelli, J. (1969): A method for including arbitrary external boundaries in the mac incompressible fluid computing technique. Journal of Computational Physics, 4:543551. 

Vorst, H. V. D. (1992). Bi-cgstab: a fast and smoothly converging variant of bi-cg for the solution of nonsynmetric linear systems. SIAM Journal Sci. Statist. Comput., 13:631-644.

Vuik, C., Saghir, A., e Boerstoel, G. P. (2000). The krylov accelerated simple(r) method for flow problems in industrial fornace. International Journal for Numerical Methods in Fluids, 33:1027-1040.

Watson, E. J. (1964). The radial spread of a liquid jet over a horinzontal plane. Journal of Fluid Mech., 20:481-499.

Watson, G. (1976). Numerical analysis. Lecture Notes in Mathematics, Springer, Berlin, 506:73-89.

Weil, If. (1940). The method of orthogonal projection in potential theory. Duke Mathematical Journal, 7:411-444.

Weinan, E. e Liu, J. (1995). Projection method I: Convergence and numerical boundary layers. Society for Industrial and Applied Mathematics Journal of Numerical and Analitical, 32:1017.

White, F. (1991). Viscous Fluid Flow. McGraw-Hill, New York.

Zhu, J. (1992). On the higher-order bounded discretization for finite volume computations of incompressible flows. Comput. Meth. Appl. Mech. Engrg., 98:345-360. 